\title{
Context-dependent, combinatorial logic of BMP signaling
}

\author{
Thesis by \\ Heidi Klumpe
}

In Partial Fulfillment of the Requirements for the

Degree of

Ph.D. in Chemical Engineering

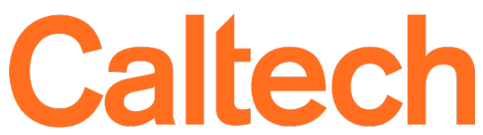

\section{CALIFORNIA INSTITUTE OF TECHNOLOGY \\ Pasadena, California}

2021

Defended May 25, 2021 
(C) 2021

Heidi Klumpe

ORCID: 0000-0001-8938-2006

Some rights reserved. This thesis is distributed under a Creative Commons Attribution-NonCommercial-ShareAlike License 


\section{ACKNOWLEDGEMENTS}

The season of graduation unexpectedly overlaps with the season of graduate school commitments, so I've recently spent many hours talking with students who are about to start what I am about to finish. I found myself saying over and over again how grateful I am to have worked with Michael Elowitz. Some trivial things are important: Michael's generosity with time and funding removed many of the stresses of graduate school, and his shrewd selection of Jo Leonardo, Leah Santat, and James Linton as effective COOs has kept the lab clean and highly functional. But at the beginning, joining the lab symbolized for me the chance to make something beautiful. And in experiments, in research questions, in scientific prose, and in figures, Michael pushed me beyond what I thought were my limits. Now, at the end of my degree, I value most what he modeled for us in priorities: creativity over cynicism, exploration over rule-following, wonder over criticism, stories over minutia, and human needs over scientific ones.

Everyone else who supported this work I did not choose to work with. They were instead gifted to me by the universe. Every day of my $\mathrm{PhD}$, I make use of a skill Yaron Antebi taught me. His wisdom and humor have carried me through many crises. James Linton reads more widely and thinks more deeply than most, and the height of his kindness makes him a statistical improbability. $\mathrm{He}$ is responsible for the omission of many biological roadblocks from this work. Christina $\mathrm{Su}$ wrote the PromiSys code that was crucial to our simulations and model fitting. As we have traveled together through DARPA reports, co-submitted manuscripts, and one volcano, her intelligence, thoughtfulness, and precision have been my guiding lights. Matthew Langley has shared the dream of BMP equivalence more closely with me than anyone else. His quick learning, deep curiosity, and cheerful support mark the outlines of an ideal colleague. Jost Vielmetter and Pamela Bjorkman generously provided the liquid-handling robot that did nearly all the pipetting reported in this thesis. Without Jost's ability to rescue robot crashes, large parts of these data would simply not exist.

Throughout this process, my thesis committee has provided crucial guidance. Dave Tirrell brought important protein intuition to my arguments around how to assess differences between BMPs. Zhen-Gang Wang bravely ventured far from his area of expertise to learn new biology and brought rigor to our modeling and quantitative analysis. Lea Goentoro consistently asked incisive questions

for interpreting results and designing experiments, while also articulating the most important issues for the project's overall meaning and significance. Rob Philips, joining in the later stages of the project, pushed for a clarity in presentation and explanation that was necessary to alchemize a heap of data into a story.

This thesis depends also on the invisible work of professors with no formal attachment to it. Justin 
Bois's BE103 class changed the way I code and teach, and was the first time at Caltech I believed in myself. The careful reader will find that all the Python code attached to this thesis resembles the syntax he teaches and should alert me to any alarming lines that do not. Samantha Butler, Karen Lyons, and Vicki Rosen are all giants in their understanding of BMP signaling in the terrifying wilderness of in vivo systems, and their feedback on presentations and our manuscript have been vital for taking the project from the theoretical to the useful.

Undergirding this thesis are a host of friends who sustained my heart and mind for the past seven years. The members of the Elowitz lab have all taught me with their powerful character and brilliance. I've had no end of companions for running, swing dancing, and improvising. To me, Avery will always be the finest house. The bedrock at the bottom of all this includes Brinda Monian, my kindred spirit, and Alessandro Zocca, mio grande gigante gentile. David, Hannah, and Hayley have been supporting this work since before I could explain why it mattered. My parents are forever the Drs. Klumpe I aspire to be, and hopefully this brings me one step closer. 


\section{ABSTRACT}

Evolution generated diverse signaling proteins for the control of multicellular patterns and organisms. These include the proteins of the Bone Morphogenetic Protein (BMP) pathway. Nearly a dozen BMPs activate the BMP pathway to promote the formation of tissues as diverse as bone, cartilage, blood vessels, and the kidney, making them attractive therapeutics for regenerating those tissues in adults. During development, the response to a given BMP depends heavily on context, such as which other BMPs are present and which BMP receptors are expressed on the cell being activated. However, despite knowing that context matters, the overall logic of this context-dependent signal processing, including the roles of specific ligands and receptors in shaping context and how this logic arises from biochemical features of specific pathway components, remains unclear. Inspired by maps of gene epistasis and drug interactions that functionally classify members of complex biological systems, we comprehensively measured responses to all pairs of ten BMP homodimers (BMP2, BMP4, BMP5, BMP6, BMP7, BMP9, BMP10, GDF5, GDF6, and GDF7), combining robotic liquid handling with a high-throughput fluorescent reporter of pathway activation. These data functionally classify ligands into "equivalence groups," or ligands that combine in the same way with all other ligands across combinations. Surprisingly, the functional groupings do not correlate with similarity of ligand sequence and can change with cell context. Together, the context-dependent equivalence groups summarize the diverse responses to combinations of BMP ligands and their dependence on specific BMP receptors. The experimentally observed pairwise responses are also consistent with a mathematical model where BMP ligands compete for limited BMP receptors with different affinities and then produce outputs with different ligand-specific activities. Ultimately, these results provide a useful reference for explaining the unique effects of BMP combinations in different tissues or time points in development, as well as highlighting counterintuitive mechanisms for this complex signal processing. Chapter 1 provides an introduction to how and why we study cell-cell signaling. Chapter 2 provides a summary of the determination of equivalence groups, their dependence on receptor context, and fitting the mathematical model of receptor competition. Chapter 3 provides suggestions for future work, including recommendations

for improved model fitting as well as crucial extensions to the definitions of BMP "combinations" and "context" to deepen our understanding and control of this critical pathway. 


\section{PUBLISHED CONTENT AND CONTRIBUTIONS}

[1] Heidi E Klumpe et al. "The context-dependent, combinatorial logic of BMP signaling". In: bioRxiv (2020). DOI: $10.1101 / 2020.12 .08 .416503$.

H.K., Y.E.A., and M.B.E. conceived and designed the experiments. H.K., M.A.L., and J.M.L. performed the experiments. H.K. and M.A.L. analyzed the experimental data. H.K., Y.E.A., and C.J.S. developed and fit the model. H.K., Y.E.A., and M.B.E wrote the paper. 


\section{TABLE OF CONTENTS}

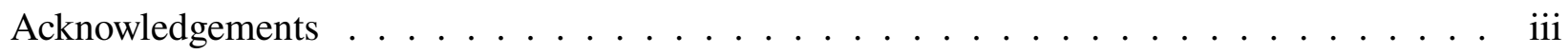

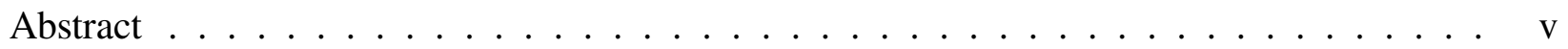

Published Content and Contributions . . . . . . . . . . . . . . . . . vi

Table of Contents . . . . . . . . . . . . . . . . . . . . . . vi

List of Figures . . . . . . . . . . . . . . . . . . . . . . . . ix

List of Tables . . . . . . . . . . . . . . . . . . . . . . . . xi

Chapter I: Introduction $\ldots \ldots \ldots \ldots \ldots \ldots$

1.1 Why study cell-cell signaling? . . . . . . . . . . . . . . . . . . 1

1.2 Why do so many proteins induce bone formation? . . . . . . . . . . . . . . 4

1.3 How do cells distinguish between very similar inputs? . . . . . . . . . . . . . . . . 6

1.4 How do we learn the "distinguishability" of BMPs? . . . . . . . . . . . . . . . . 8

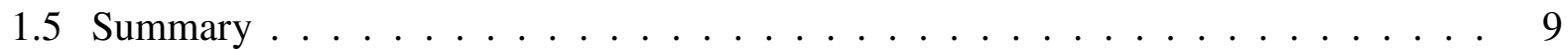

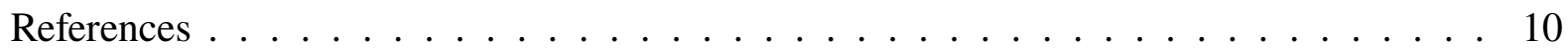

Chapter II: Combinatorial, context-dependent logic of BMP signaling . . . . . . . . . . . 17

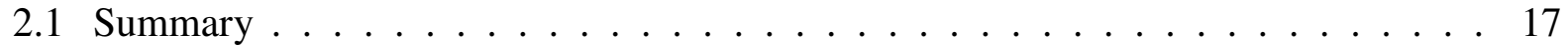

2.2 Introduction $\ldots \ldots \ldots \ldots \ldots \ldots \ldots \ldots$

2.3 Results . . . . . . . . . . . . . . . . . . . . 20

2.3.1 Quantitative dose-response measurements reveal ligand-specific features. . 20

2.3 .2 Ligands exhibit diverse combinatorial profiles. . . . . . . . . . . . . . 21

2.3.3 Ligand interactions reveal BMP equivalence groups in NMuMG cells. . . . 24

2.3 .4 Ligand equivalence depends on cell context. . . . . . . . . . . . . . . 27

$2.3 .5 \quad$ ACVR1 knockdown recapitulates features of mESC ligand interactions. . . 29

2.3.6 Perturbations of multiple BMP receptors reveal flexibility of ligand equiv-

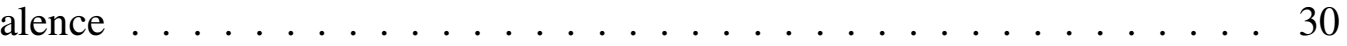

2.3.7 Comparing signaling across cell types reveals global structure of ligand

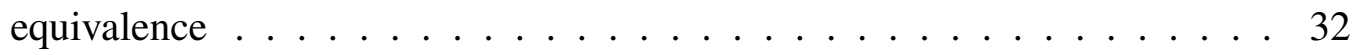

2.3.8 A mathematical model of competitive ligand-receptor interactions can explain context-dependent ligand interactions . . . . . . . . . . . . 34

2.3 .9 In the model, context-dependence emerges from redistribution of signaling

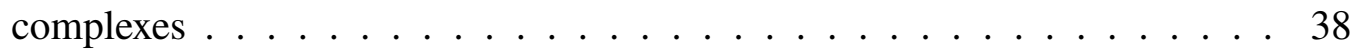

2.4 Discussion $\ldots \ldots \ldots \ldots \ldots \ldots \ldots$

2.5 Acknowledgments . . . . . . . . . . . . . . . . . . . . . . . . . . . . . . . . . . . . . .

2.6 Supplementary Figures $\ldots \ldots \ldots$. . . . . . . . . . . . . . . . . . . . . . . . . . . . . . . . . . . . . . . . .

2.7 STAR Methods . . . . . . . . . . . . . . . . . . . . . . . . . . . . . . . . . . . . . . . . . . . .

2.7 .1 Resource Availability . . . . . . . . . . . . . . . . . . . . . . . . . . . . . . 57

2.7 .2 Experimental Model and Subject Details . . . . . . . . . . . . . . . . . . . . . . . . . 58

2.7 .3 Method Details . . . . . . . . . . . . . . . . . 59

2.7 .4 Quantification and Statistical Analysis . . . . . . . . . . . . . . . . . 61

2.8 Key Resources and Tables . . . . . . . . . . . . . . . . . . . . 73 
References ............................. 76 Chapter III: Expanding the predictive understanding of BMP signaling to new combinations

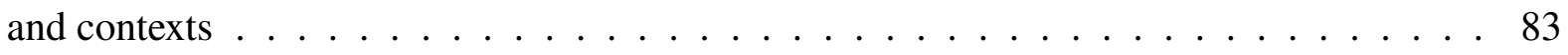

3.1 Summary . . . . . . . . . . . . . . . . . . . . 83

3.2 Limitations of existing BMP model require more data or alternative model architecture. 83

3.2.1 Data collected at saturating ligand concentrations improves quality of fits but does not capture all pairwise interactions. . . . . . . . . . . . . . 84

3.2 .2 Diverse paraemter estimates fit the data equally well. . . . . . . . . . . 85

3.2.3 In analytical model, extreme receptor ratios or very low affinities are harder

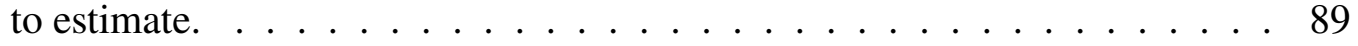

3.2.4 Ligand combinations and receptor perturbations constrain parameters more than individual dose responses or cell lines alone. . . . . . . . . . . . 94

3.2 .5 Future parameter fitting should focus on ACVR2A and ACVR2B overexpression, with possible BMPR2 knockdown. . . . . . . . . . . . 98

3.2 .6 Two-step model provides an alternative and more physically accurate model

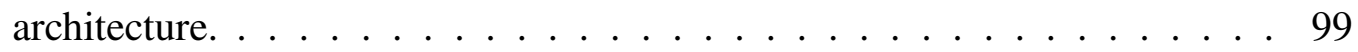

3.2.7 Ligand-specific differences in affinity and receptor-specific differences in activity may be measured directly. . . . . . . . . . . . . . . . 102

3.3 Connecting simple ligand pairs and single receptor perturbations to the larger world

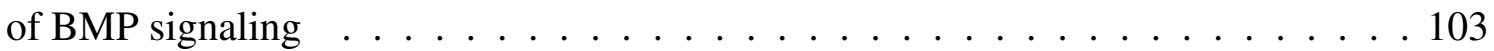

3.3.1 Responses to combinations of more than two ligands can have non-trivial differences from pairwise responses. . . . . . . . . . . . . . . 104

3.3.2 $\quad$ BMP heterodimers and chimeras represent novel competitors for BMP

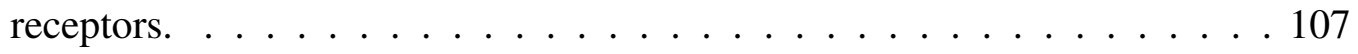

3.3.3 BMPs may combine in unique ways with other molecules that share a subset of BMP receptors. . . . . . . . . . . . . . . . . . . . . . . 109

3.3 .4 Perturbations of other pathway components can produce a new cell context

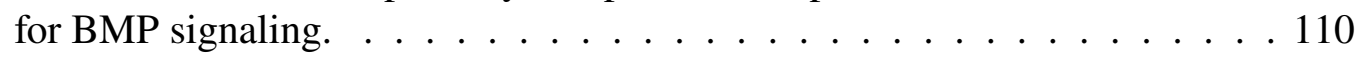

3.3 .5 Receptor-ligand computations govern other immediate outputs and down-

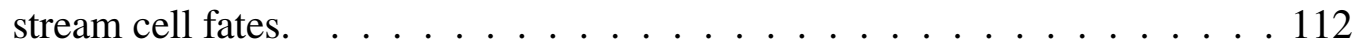

3.4 Supplementary Figures $\ldots \ldots \ldots$. . . . . . . . . . . . . . . . . . . . . . . 114

References . . . . . . . . . . . . . . . . . . . . . 116 


\section{LIST OF FIGURES}

Number Page

$2.1 \quad$ BMP ligands can be classified by their pairwise interactions . . . . . . . . . . . . 19

2.2 Pairwise titration of relative ligand concentrations reveals ligand interactions . . . . 22

2.3 Antagonism and suppression define BMP equivalence groups in NMuMG cells . . . 24

2.4 Mouse ES cells exhibit distinct BMP equivalence groups . . . . . . . . . . . . . 27

2.5 ACVR1 knockdown reveals the effects of receptor context on ligand equivalence . . 30

2.6 Analysis of multiple cell contexts reveals global ligand equivalence groups . . . . . 32

2.7 Mathematical model of receptor competition can explain contextual ligand equiva-

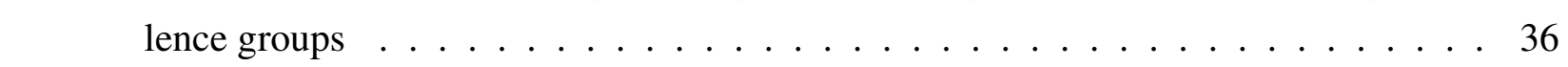

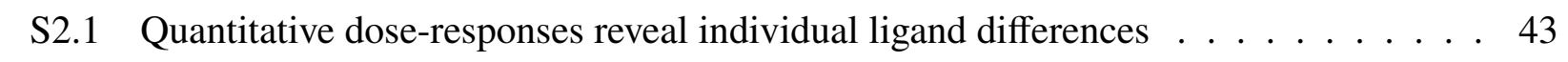

\begin{tabular}{|ll|}
\hline S2.2 & Interaction Coefficient (IC) classifies the range of pairwise interactions observed in \\
\hline
\end{tabular} simulations and experiments . . . . . . . . . . . . . . 45

S2.3 Full quantification of BMP signaling in mESC reveals unique ligand properties . . . 47

S2.4 Knockdown of ACVR1 by shRNA phenocopies siRNA knockdown and alters pairwise interactions . . . . . . . . . . . . . . . . . . . 49

S2.5 Constitutive shRNA expression knocks down BMPR2 and BMPR1A to produce receptor-specific effects distinct from ACVR1 knockdown . . . . . . . . . . . . . 51

S2.6 Ectopic expression of missing BMP receptors increases frequency of additive interactions and simplifies ligand equivalence . . . . . . . . . . . . . . 53

S2.7 $\quad$ BMP ligand sequence differences correlate with global functional differences . . . . 55

S2.8 In the model, abundance of high- and low-activity complexes depend on affinities, receptor context, and ligand context . . . . . . . . . . . . . . 56 56

$3.1 \quad$ Fitting dose responses alongside rim data improves model performance at subsaturating ligand concentrations. . . . . . . . . . . . . . . . 86

3.2 Parameter fits are more sensitive to receptor rescaling ratios and affinities than activities. 87

3.3 Parameters of simple one-step model can be fit within 2-fold error for nearly all parameter sets. . . . . . . . . . . . . . . . . . 91

3.4 Very low affinities and more extreme receptor ratios produce less accurate parameter estimates. . . . . . . . . . . . . . . . . . 93

3.5 Pairwise data constrains parameter fits more than dose responses alone. . . . . . . . 96

3.6 Receptor perturbation data constrains parameters for unrelated receptors. . . . . . . 97

3.7 Published data show order of magnitude differences in ligand-receptor affinities. . . 99 
3.8 Best fit estimates of two-step model parameters have large errors. . . . . . . . . . . 101

3.9 One-step model provides better fits of most simulated data, whether generated from one-step or two-step model . . . . . . . . . . . . . . . . . . . . . 102

3.10 Signaling complex formation, Smad activation, and downstream transciptional outputs vary between BMP signaling contexts. . . . . . . . . . . . . . . . . 110

S3.1 Parameters estimated by fitting either pairwise data and dose responses or pairwise data alone are qualitatively similar . . . . . . . . . . . . . . . . . 114

S3.2 Very low affinity values harder to estimate than very low activity values. . . . . . . . 116 


\section{LIST OF TABLES}

Number Page

2.1 Key Resources . . . . . . . . . . . . . . . . . . . . . . . . 73

2.2 Recombinant BMP Ligands and Concentrations . . . . . . . . . . . . . . . . . . . 74

2.3 shRNA Sequences . . . . . . . . . . . . . . . . . . 74

2.4 siRNA Sequences $\ldots \ldots \ldots \ldots \ldots \ldots$

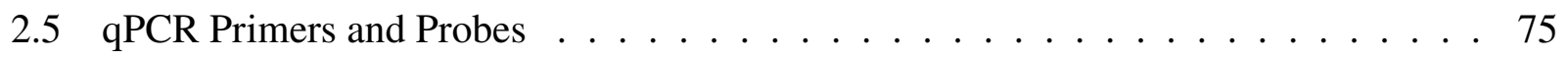


Chapter 1

\section{INTRODUCTION}

\subsection{Why study cell-cell signaling?}

The human body is a remarkable consortium of diverse cell types that collectively extract energy, fight infection, heal wounds, and excrete waste. Both the production and placement of these many cell types are crucial for proper multicellular function. For example, folds of epithelium, as in hair follicles and intestincal crypts, serve as reservoirs for pluripotent stem cells that consistently maintain these tissues with high turnover ${ }^{18135}$ In joints, synovial fluid and cartilage placed between larger bones ease their relative movement. ${ }^{64}$ The formation of branched tubes allows liquids to be delivered or filtered throughout the body, a structure that is repeated in blood vessels, kidney ducts, lung alveoli, and salivary and mammary glands. ${ }^{55}$ Thus, managing which cell types form where is key to many of the wonders of multicellular machines.

Cell-cell signaling is a key driver of these processes. During development, extracellular inputs called signaling proteins coordinate cell fates and locations across the embryo. Signaling proteins are grouped by the changes they produce inside the cell, which is usually the activation of a specific signaling pathway. For example, signaling proteins from the Bone Morphogenetic Protein (BMP) pathway bind BMP receptors to activate transcription factors called Smad1, Smad5, and Smad8 (i.e. Smad1/5/8). The amount of BMP signaling protein in a certain part of the embryo controls the level of pathway activation and therefore the size or type of the response in the exposed cells. In zebrafish embryos, a high concentration of BMP signaling protein on one side of the embryo produces the cell types associated with the ventral side, while medium and low BMP concentrations produce different cell types, associated with other points on the dorso-ventral axis. ${ }^{27}$ Signaling proteins from the Fibroblast Growth Factor (FGF) pathway attract the "tip" cells at the edge of a new blood vessel branch, while Notch signaling proteins expressed in the "tip" cell instruct adjacent to become "stalk" cells important for lengthening the branch. ${ }^{55}$ In this way, the concentrations and identities of signaling proteins can control which cell types form where, producing the complex and functional structures like blood vessels.

The ability to understand and control how these structures arise is essential to repair and build such biological machines. However, while it is known that signaling pathways produce cell fates and important patterns, we do not yet know all the details of how. Beyond knowing which molecules are important in which developing tissues, a full understanding of cell-cell signaling could describe what information is conveyed by that pathway (e.g. messages telling a cell to proliferate, differentiate, or 
remain pluripotent), which molecules carry that signal, and how other molecules decode or modify that signal. To demonstrate how increasing our understanding of pathway function provides tools to describe, predict, and engineer developmental phenomena, we can consider the history of the Notch signaling pathway.

In the early 1910s, the Notch locus was named for the notched Drosophila wings that developed when the locus was mutated ${ }^{48}$ However, it was not clear what this protein's coding sequence was, or how it exerted its effects on Drosophila wings. Only in the 1980s was this "Notch" locus grouped with other genomic locations that affected cell fates in the same powerful way: biasing Drosophila ectoderm cells toward a neural, instead of epidermal, fate. ${ }^{42}$ Identifying these genomic loci was a powerful discovery when coupled with other biological knowledge. Scientists, armed with better knowledge of codons and coding sequences, could identify the amino acid sequences of the fate-determining genes and compare them with other genes that had been discovered. 39 Recurring functional motifs (i.e. similar amino acid sequences in genes of known function) revealed that the Notch pathway components resembled other ligands, receptors, and intercellular components that could bind DNA. ${ }^{[}$These predicted functions indicated how Notch ligands outside the cell could cause an internal change in cell state, suggesting that extracellular ligands could bind receptors on the cell surface to activate downstream genes the defined the cell fate. Closer studies of these proteins revealed functional sub-domains in their sequences, which explained some of Notch's peculiarities. Ligands had a transmembrane domain, indicating they were membranebound, which explained why they produced their effects on short ranges. ${ }^{32}$ The Notch receptor also contained an intracellular domain that was cleaved when the receptor was activated, producing a protein fragment that could enter the nucleus and activate gene expression. $\frac{69}{6}$

In addition to understanding the rudimentary steps of Notch activation, scientists sought to understand how these binding and activation events produced the effects observed in development. One mystery was how Notch produced such diverse effects, since mutating the Notch pathway components disrupted the formation of many tissues beyond those in which Notch was discovered ${ }^{6}$ Scientists soon identified chemical modifications that governed receptor activation and internalization, as well as the large number of proteins, beyond the ligand and receptor, that were required to activate gene expression. 11 In addition, there were even more pathway components than originally thought, including additional ligand and receptor variants that could bind each other in any ligand-receptor pair to cleave the receptor's intracellular domain. $\stackrel{38}{3}$ However, these components, despite being variants of the same pathway component, had subtle differences, such as different affinities for the receptor, ${ }^{2]}$ differences in ligand-receptor complex structure, $\stackrel{44[43}{ }$ different sensitivity to Fringe proteins that modulated pathway activation by adding sugars to the receptors, 41 and different dynamics of pathway activation that promoted different fates. $\stackrel{51}{\underline{ }}$ These subtler and 
harder-to-discover features of the signaling proteins were important details in the overall picture of how Notch activation orchestrates a diverse array of cell fates. Moreover, they inspired engineering of new signaling systems. The cleavage site governing Notch activation allows the receptor to be repurposed as a synthetic sensor, where binding of the extracellular domain to a target of interest releases any activation domain of choice, ${ }^{49}$ which has been used to program specialized T-cells for targeting tumors ${ }^{63 \mid 17}$ and even SARS-CoV-2! 76

This example of the Notch pathway reveals the trends and impact of cell signaling research. First, functional classification is important to tame the inevitable complexity. Separating the many molecules known to be "important" for determining cell fate into ligands, receptors, and intracellular effectors simplified an ever-growing list of proteins into clear groups with well-defined interactions: extracellular ligands binding membrane-bound receptors to activate intracellular changes and downstream gene expression. This separation of extracellular, membrane-level, and intracellular signaling events also ordered the effects of cell signaling logically in time, showing how classification schemes can provide insight into a sytem, rather than merely organizing it like a stamp collection.

Second, new breakthroughs in Notch narrowed from the general to the specific. The earliest work focused on identifying higher-level events in development that were affected by Notch mutations. This prompted the discovery of the specific binding steps that linked extracellular signals to cell fate choices. However, this too became more specific, as these binding events that convey information across membranes can proceed in subtly different ways, as evidenced by the diverse effects of Notch signaling in development. This required a more specific understanding of the molecules that transduced Notch signaling, and the range of each molecule's behaviors. Ultimately, a change in the shape of a fly wing that was visible to the naked eye sparked a set of discoveries about the pathway's sensitivity to the addition or removal of sugar groups containing merely a dozen atoms.

Lastly, the history of Notch shows when and why narrow results are useful parts of our understanding. Smaller discoveries can increase our ability to control the pathway when they connect those details to overall function. Determining the distinct affinities and dynamics of Notch ligands allows scientists to rationally direct cell fates with different ligands. $\frac{51}{1}$ The discovery that receptor activation cleaved a portion of the Notch receptor allowed more precise control of T-cell activation with engineered, synthetic Notch receptors. ${ }^{63 \mid 17}$ Seemingly small discoveries also have outsized effects when they provide a new interpretation of existing data. Identifying functional ligand differences in vitro implies that they can govern different processes in vivo, where they may already be known to be differentially expressed. Moreover, the discovery that the Notch ligands were membrane-bound explained their previously observed short-range effects.

With this history in mind, this thesis aims to provide actionable information about cell-cell signaling. 
Here, we focus on signalling at the level of the membrane, the intermediate step between extracellular production of signal and intracellular effects on gene expression and cell fate. Rather than Notch, we take the BMP pathway as our model system. Some of what we observe is the result of the BMP pathway's unique traits. Unlike Notch, the BMP ligands are not membrane-bound and can therefore diffuse freely. Also, a full BMP signaling complex requires two types of receptor, not just one. But how BMP conveys information across the membrane via ligand-receptor interactions is generally relevant for the many other pathways (e.g. Notch, FGF, Wnt, TGF- $\beta$ ) that are activated by complex ligand-receptor interactions.

Also, as anticipated above, we provide a relatively narrow result, showing the combinatorial properties of BMP ligands when signaling in only a few receptor environments. However, by providing a functional classification of ligand differences, the BMP "equivalence groups" can help tame the complexity of BMP signaling in development. Moreover, they provide possible explanations of previously observed differences in where BMP ligands are expressed and how they behave in combinations. Lastly, these results provide a small step toward tying small molecular details, such as differences in ligand affinity and activity, back to higher-level properties, such as the frequency of non-additive interactions between ligands or the flexibility of changes in ligand responsiveness. Overall, by connecting to previous work and providing directions for future research, the ligand equivalence groups described here are a small step in the ongoing quest to control cell fates, which began with the mystical discovery of a biological material that produced bone in unexpected locations.

\subsection{Why do so many proteins induce bone formation?}

The existence of a mysterious, bone-inducing biological material was first reported in 1965. Marshall Urist discovered that demineralized bone matrix, when injected into an adult animal, could promote bone growth. ${ }^{74}$ Urist later theorized that the matrix contained a protein (or proteins) with "Bone Morphogenetic Protein" properties. ${ }^{[5}$ Researchers spent over fifteen years optimizing the purification of active protein from the dense bone matrix. .665 Amino acid sequences determined from these purified protein samples allowed researchers to rapidly isolate genomic DNA (in the pre-genome sequencing era) that encoded those protein sequences. ${ }^{78}$ Surprisingly, the purified protein samples contained more than one BMP, and the number of known BMPs grew from the (technically) two reported in 1988 to at least a dozen when BMP9 and BMP10 were reported in $1995.78|13| 59|60| 12|72| 14$ The sequences of these proteins were very similar, which was leveraged to discover more lowly expressed BMPs. Probes for BMP4 and BMP7 were used to identify BMP8, BMP9, and BMP10. 12|14|60

The increasing number of BMP ligands prompted a question: do all these proteins in this mixture 
produce their effects (i.e. the induction of bone and cartilage) in the same manner? BMP1 was among the BMPs first discovered in 1988 but had a very different amino acid sequence. ${ }^{78}$ It was later revealed to be a protease that cleaved collagen and also activated TGF- $\beta$ ligands. ${ }^{36 \mid 24}$ However, the remaining BMPs were highly similar to each other and resembled the more well-known TGF- $\beta$ ligands. Indeed, this resemblance indicated the similarity of their biochemistry. All the remaining BMPs were classified as part of the TGF- $\beta$ superfamily of signaling proteins. Every ligand in the superfamily could bind a common pool of Type I and Type II receptors, and a ligand bringing these receptors into close proximity produced phosphorylated R-Smad (i.e. receptor Smads). However, the TGF- $\beta$ superfamily ligands came in two flavors, depending on which R-Smads they activated: Smad1/5/8 or Smad2/3. For the most part, the newly discovered BMPs activated Smad1/5/8. 50

Thus, the BMPs had similar sequences, bound the same receptors, and activated the same Smad1/5/8 output. Unlike BMP1, they appeared to induce bone formation in the same way, implying they were functionally redundant. Experiments studying the roles of BMPs in vivo also implied that the signaling proteins had the same function overall. One common result was to find that, while many BMPs were expressed in a developing tissue, knocking out an individual BMP rarely had an effect. This implied that the other BMPs present in the tissue compensated for the loss of BMP. For example, BMP6 is expressed in developing kidney, but knocking out BMP6 does not disrupt kidney formation, 25 and knocking out BMP7 or BMP4 during limb development does not alter limb formation. ${ }^{10}$ A 1999 review of BMP signaling in the kidney bemoans the "[f] unctional redundancy among BMP family members" that made it difficult to determine the roles of BMP signaling and different ligands with knockout studies. ${ }^{[25}$ Surprisingly, containing multiple ligands that bind the same receptors to activate the same output is not unique to the BMP pathway. For example, the EGF pathway has 7 ligands, 29 the Wnt pathway has 10 ligands,, 54 and the FGF pathway has 15 canonical ligands. ${ }^{56}$ In each case, these ligands have similar sequences, bind the same or similar receptors, and activate the same downstream outputs.

However, other pieces of evidence suggest that BMPs may not be perfect replacements for each other. Knocking out BMP7 produces lethal kidney failure, but knocking out BMP5 produces only a swollen kidney due to insufficient drainage. ${ }^{25}$ In the absence of BMP2, knocking out BMP4 completely disrupts limb formation, while the BMP7 knockout has only modest effects ${ }^{[10}$ Even more strikingly, one BMP knocked into the locus of another cannot necessarily reverse the effects of removing the BMP that it replaces. This implies that one BMP, even when expressed at the same location, time, and concentration as a very similar BMP, cannot replace it in all cases. Specifically, BMP9 can rescue BMP10's role in angiogenesis, but not heart formation, 16 while BMP4 can replace BMP7 in kidney formation, but not eye development. ${ }^{58}$ Even at the time BMP9 was discovered, scientists noted it could only induce bone formation at much higher concentrations than other BMPs, 
suggesting it had unique functional roles. ${ }^{12}$ Thus, two things appear simultaneously true: BMP ligands can redundantly compensate for each other, but they can also have unique roles. Which statement applies depends on the cell context and which two BMP ligands are being considered.

Overall, such context-dependent ligand redundancy is consistent with biological intuition. First, having multiple copies of a gene protects an organism from mutations in any one copy, but maintaining those redundant parts introduces an additional energetic cost. ${ }^{20}$ Only for some parts is that additional energetic cost outweighed by the benefits of redundancy, analogous to the way JPL engineers could load the Perseverance rover with duplicate electronics, but only one $150 \mathrm{lb}$ parachute. ${ }^{3[52}$ Given the presumably large cost of expressing multiple versions of the same protein, it seems likely that additional BMPs perform at least some non-redundant functions to justify their maintenance in the genome. Second, early multicellular organisms pattern their embryos with a simpler version of BMP signaling, which includes only three ligands or fewer. ${ }^{40[47 \mid 61}$ The number of BMPs expressed by an organism tends to increase with the complexity of the organism, further implying that the diversity of BMPs provides additional functionality.

Nonetheless, the answer "it's complicated" to a question of biological mystery can be dissatisfying, even if true. To go farther, we can piece together the rules of this complexity by identifying patterns in what cells are doing. These patterns can then help us identify which mechanisms could generate this capricious, but ultimately rule-following, behavior.

\subsection{How do cells distinguish between very similar inputs?}

The mystery before us is how BMP ligands, by binding the same sets of receptors to activate the same Smad1/5/8 transcription factors, manage to play such different roles in development. One straightforward explanation is that all ligands are equivalent but expressed at different times and places in the developing embryo. For example, mutations in GDF5 and BMP5 disrupt the condensation of mesenchyme cells in a similar manner, but in different skeletal elements, generating the hypothesis that " $[\mathrm{t}]$ he skeleton of higher animals thus appears to be a mosaic structure that is built from the composite patterns of activity of different BMP-like proteins.' ${ }^{, 72}$ Alternatively, different BMPs may vary only in their potency. In this view, the different effects of knocking out different BMPs just reflect the ligands' relative strengths, but at sufficiently high concentrations, all BMPs could perform the same roles.

These simple hypotheses may explain some observed ligand differences, but they cannot explain BMP9's context-dependent replacement of BMP10, where the expression of the (presumably) same number of molecules at the same time and location has different effects depending on the tissue. Therefore, as recently as 2019, a review stated that this discrepancy between functional differences and biochemical similarity showed that "our current view of TGF $\beta$ signaling initiation just by 
hetero-oligomerization of two receptor subtypes and transduction via two main pathways in an on-off switch manner is too simplified., 53

What this simple view of BMP signaling is missing may be found in our understanding of other signaling pathways. Like BMP, other signaling pathways convey information across membranes by ligands binding receptors at the membrane to produce intracellular effects. Many of these pathways use these ligand-receptor interactions to distinguish between their many ligands. For example, ligands can have small but subtle differences in how they bind to receptor variants. In this way, ligands can produce distinct signaling complexes that generate different outputs, such as Wnt7's use of a ligand-specific co-receptor, $\stackrel{21}{21}$ or VEGF ligands' preferences for different VEGF receptor variants, ${ }^{71}$ or different interleukins' unequal competition for a shared receptor subunit. ${ }^{26}$ Ligands can also be perceived differently through the same receptor. Ligands may produce different absolute activation levels because they stabilize the signaling complex for different durations, $\stackrel{1 / 23}{ }$ produce different sizes of receptor clusters,,$\sqrt[51 \mid 62]{ }$ preserve the signaling complex for different time periods in intracellular vesicles, $\stackrel{[22}{2}$ or cause the receptor to take on unique conformations biased to different outputs. ${ }^{77}$

The BMP pathway has features consistent with many of these mechanisms. Like the interleukins competing for a shared receptor, BMP ligands compete for the various BMP receptors with different affinities. ${ }^{31}$ Indeed, the ten most common BMP homodimers are often classified into four groups (BMP2/4, BMP5/6/7, BMP9/10, GDF5/6/7) defined by different receptor preferences. ${ }^{[57}$ More recent evidence has shown that different BMP ligand-receptor signaling complexes may also have different activities. Activin-A produces non-signaling complexes with ACVR1,,$\frac{8}{\text { and knocking }}$ down BMPR2 can increase pathway activation by some BMPs, $\stackrel{28 \mid 33}{2}$ implying BMPR2 forms low activity complexes. Active BMP signaling complexes can also include co-receptors, such as endoglin, MuSK, and Repulsive Guidance Molecules.9|15/80 BMP receptors are also internalized for sustained signaling or receptor degradation. $\frac{30 \mid 37}{}$ Lastly, while all complexes can activate the same pool of R-Smads, collectively referred to as Smad1/5/8, certain complexes may activate different amounts of Smad1 or Smad5, or even other non-canonical outputs $\underline{68}$

A recent mathematical model of BMP signaling combined three of these features to show how BMP ligands could be context-dependent replacements for each other ${ }^{4}$ In the model, each ligand had a unique affinity for each receptor dimer, ligands competed for shared receptors limited by a mass balance constraint, and each resulting signaling complex produced the same output but with different activities. Because ligands compete for and activate receptors in distinct ways, they could not replace each other in all contexts, particularly when competing with another ligand for receptors or when activating cells types that expressed very different BMP receptors. The model predicted BMP ligands could combine non-additively to activate the BMP pathway, and the type of pairwise 
interaction between two ligands could change in a new cell type, both of which were observed in vitro. $\underline{4}$

While this model does not include all the features responsible for producing diverse effects in vivo, it shows that the ligands' different affinities and activities are sufficient to generate ligand-specific responses that change with cell context. Unfortunately, most of the ligand-receptor affinities and activities are not known. This is in part because promiscuous ligand-receptor binding means there are a large number of these interactions, and directly measuring biochemical properties is difficult to achieve in high-throughput. Thus, while the model has significant explanatory power, it does not yet indicate which BMPs could be good replacements for each other and how these relationships vary in the presence of different BMPs and BMP receptors. We therefore require alternative methods for identifying functional differences that emerge from these underlying biochemical differences.

\subsection{How do we learn the "distinguishability" of BMPs?}

Genetic studies of BMP knockouts and the discovery of non-additive pairwise interactions between BMPs suggest that these ligands can be distinguished in certain contexts and perform distinct roles. However, these roles depend on ligand affinities and activities, parameters which are difficult to comprehensively measure. $\sqrt[7 / 31 / 34 / 67]{ }$ Therefore, for my thesis, we take the inverse approach. Rather than using parameters to predict the pairwise interactions, the presence of pairwise interactions can be used to infer differences in parameters. Moreover, these pairwise interactions, like the biochemical parameters, can shed light on the combinatorial, context-dependent roles of BMPs ligands that have been observed in development.

This approach to classifying implicit biochemical differences by measuring their explicit effects on cell behavior has been used for other biological networks with large numbers of interacting components. For example, antibiotics reduce bacterial growth rate by inhibiting different essential proteins. Grouping antibiotics by their pairwise effects on overall growth rate recapitulated their groupings by mechanism. 79 Similarly, grouping genes by their pairwise epistatic effects revealed groups of genes known to be in the same cellular compartment or involved in the same protein complex or cellular process. $\frac{19}{19}$ Thus, in each case, the phenotypic effect of pairwise interactions revealed underlying differences in biochemistry or binding. In the same way, analyzing responses to pairs of BMP ligands can reveal the functional differences that emerge from underlying differences in affinities or activities, even if those affinities and activities are not known. Thus, the pairwise interactions provide another path to functionally classifying BMP ligands, and perhaps explaining their distinct roles in development. This classification could also guide the design of chimeric BMPs, which attempt to fuse the properties of multiple BMPs to produce more effective therapies. 70 Identifying the context-dependent and combinatorial effects of BMP ligands via their pairwise 
interactions also lays the groundwork for future studies of how BMP ligands produce these diverse effects. We hypothesize that these differences largely depend on the ligands' differences of activity and affinity. Showing that a mathematical model incorporating only these elements can produce the complex effects observed in the data provides evidence that this mechanism is a possibly complete explanation, and we therefore explored fitting such a model to our data. However, significantly more work is required to improve and validate this model. For example, future experiments could also focus on measuring affinities and activities more directly. The BMP model employed here ${ }^{73}$ could be expanded to include the two-step binding necessary to assemble an active signaling complex as well as the dimeric nature of the ligands, which can recruit four receptors in total. The effects of many other upstream and downstream signaling effectors, such as extracellular antagonists that selectively block certain BMPs' activities or intracellular differences in chromatin state that determine which genes are activated the Smad1/5/8 phosphorylation, could also be included..$^{46}$ Therefore, alternative or more detailed models ${ }^{45}$ of BMP signaling, as well as further experiments, are necessary to understand the full set of factors producing the diverse and powerful effects of BMP signaling.

\subsection{Summary}

Signaling between cells provides important information for cell fate and organismal patterning. Analyzing the capabilities of signaling pathways provides tools to understand and engineer those fates and patterns. Cell signaling is implemented by a variety of pathways, each with unique ligand and receptor protein sequences as well as biochemistry of complex formation and activation. These include the BMP, Notch, FGF, and Wnt pathways, which can similarly convey information across the cell membrane with their own unique mechanisms. The BMP pathway in particular has the general features of cell-cell signaling, where extracellular ligands bind and activate receptors that induce downstream gene expression, as well as its own unique features of freely-diffusing ligands that form heterodimers of Type I and Type II receptors, which can then activate Smad1/5/8 transcription factors.

A striking feature of the BMP pathway is the vast number of ligands that can bind the BMP receptors to activate pathway output, similar to other pathways that can be activated by any one of many ligand variants, such as the $10 \mathrm{Wnt}$ ligands $\stackrel{54}{5}$ and 15 FGF ligands. ${ }^{56}$ Removing or adding BMP ligands during development reveals that they have non-redundant functions that depend on cell context. Therefore, the BMP pathway is an excellent model system for studying how a diverse set of ligands from the same pathways can produce different context-dependent effects.

In the BMP pathway, ligands compete for a shared receptor pool via their different affinities, and produce output with different activities. This can generate non-additive pairwise interactions be- 
tween ligands that change with cell context, which could explain the context-dependent redundancy of BMP ligands observed in development. However, without knowing the precise biochemical parameters, such as affinities and activities, that produce these non-additive interactions, it is not possible to predict the precise patterns of redundant or unique ligands, and how these vary with cell context. Instead, extensively measuring their pairwise interactions, which emerge from their unique biochemical properties, can reveal these relationships.

In Chapter 2 of this thesis, we present the comprehensive measurement of responses to all pairs of 10 BMP ligands across seven cell lines. These results show that the complexity of BMP ligands can be simplified into equivalence groups, where members of the same equivalence group can replace each other as individuals or in any combination. These equivalence groups do not match similarity groupings based on ligand protein sequence or supposed receptor preferences. Since redundancy relationships can change between developmental contexts, we asked if equivalence groups changed in a new cell line. Indeed, measuring pairwise interactions in a different cell line produced a new ligand classification, and recapitulating some of the cell lines' differences in receptor expression could also change ligand classification in a similar manner. Thus, these results support BMP receptor expression as a strong driver of context-dependent ligand behavior. Pairwise data collected across many receptor contexts showed a global equivalence map, where some BMP ligands appear to be completely redundant across all contexts, whereas others have non-additive interactions in nearly all contexts. Overall, these results provide useful results for predicting the responses to combinations of BMPs in various contexts and how activity is perturbed by adding or removing a BMP ligand. Lastly, as explored in Chapter 3, these findings can also constrain parameter fits that attempt to describe the biochemical parameters underpinning these functional differences.

\section{References}

[1] Fozia Ahmed et al. "The Biased Ligands NGF and NT-3 Differentially Stabilize Trk-A Dimers". en. In: Biophys. J. 120.1 (Jan. 2021), pp. 55-63. ISSN: 0006-3495, 1542-0086. DOI: $10.1016 /$ j.bpj.2020.11.2262.

[2] Marie Blanke Andrawes et al. "Intrinsic selectivity of Notch 1 for Delta-like 4 over Deltalike 1". en. In: J. Biol. Chem. 288.35 (Aug. 2013), pp. 25477-25489. ISSN: 0021-9258, 1083-351X. DOI: $10.1074 / \mathrm{jbc} . \mathrm{M113.454850.}$

[3] Answering Your Questions: Will the 2020 Mars Rover Have Back-Up Features? https: //wWw . techbriefs.com/component/content/article/tb/stories/blog/37178. Accessed: 2021-3-18. July 2020. 
[4] Yaron E Antebi et al. "Combinatorial Signal Perception in the BMP Pathway". en. In: Cell 170.6 (Sept. 2017), 1184-1196.e24. ISSN: 0092-8674, 1097-4172. DOI: 10.1016/j . cell. 2017.08 .015 .

[5] S Artavanis-Tsakonas, K Matsuno, and M E Fortini. "Notch signaling". en. In: Science 268.5208 (Apr. 1995), pp. 225-232. ISSN: 0036-8075. DOI: 10.1126/science.7716513.

[6] S Artavanis-Tsakonas, M D Rand, and R J Lake. "Notch signaling: cell fate control and signal integration in development”. en. In: Science 284.5415 (Apr. 1999), pp. 770-776. ISSN: 0036-8075. DOI: $10.1126 /$ science.284.5415.770.

[7] Senem Aykul and Erik Martinez-Hackert. "Transforming Growth Factor- $\beta$ Family Ligands Can Function as Antagonists by Competing for Type II Receptor Binding”. en. In: J. Biol. Chem. 291.20 (May 2016), pp. 10792-10804. ISSN: 0021-9258, 1083-351X. DOI: 10.1074/ jbc.M115.713487.

[8] Senem Aykul et al. "Activin A forms a non-signaling complex with ACVR1 and type II Activin/BMP receptors via its finger 2 tip loop”. en. In: Elife 9 (June 2020). ISSN: 2050084X. DOI: $10.7554 /$ eLife. 54582 .

[9] Jodie L Babitt et al. "Repulsive guidance molecule (RGMa), a DRAGON homologue, is a bone morphogenetic protein co-receptor". en. In: J. Biol. Chem. 280.33 (Aug. 2005), pp. 29820-29827. ISSN: 0021-9258. DOI: $10.1074 /$ jbc . M503511200.

[10] Amitabha Bandyopadhyay et al. "Genetic analysis of the roles of BMP2, BMP4, and BMP7 in limb patterning and skeletogenesis". en. In: PLoS Genet. 2.12 (Dec. 2006), e216. ISSN: 1553-7390, 1553-7404. Dor: 10.1371/journal.pgen.0020216.

[11] Sarah J Bray. "Notch signalling: a simple pathway becomes complex". en. In: Nat. Rev. Mol. Cell Biol. 7.9 (Sept. 2006), pp. 678-689. ISSN: 1471-0072. DoI: 10.1038/nrm2009.

[12] A J Celeste et al. "BONE MORPHOGENETIC PROTEIN-9, A NEW MEMBER OF THE TGF- $\beta$ SUPERFAMILY”. In: J. Bone Miner. Res. 9.Supplement 1 (1994), S136. ISSN: 08840431.

[13] A J Celeste et al. "Identification of transforming growth factor beta family members present in bone-inductive protein purified from bovine bone". en. In: Proc. Natl. Acad. Sci. U. S. A. 87.24 (Dec. 1990), pp. 9843-9847. ISSN: 0027-8424. DOI: 10.1073/pnas .87.24.9843.

[14] A J Celeste et al. "The molecular cloning of human bone morphogenetic proteins-10,-11, and -12 , three new members of the transforming growth factor- $\beta$ superfamily". In: J. Bone Miner. Res. 10.Supplement 1 (1995), S336. ISSN: 0884-0431.

[15] S Cheifetz et al. "Endoglin is a component of the transforming growth factor-beta receptor system in human endothelial cells". en. In: J. Biol. Chem. 267.27 (Sept. 1992), pp. 1902719030. ISSN: 0021-9258.

[16] H Chen et al. "Context-dependent signaling defines roles of BMP9 and BMP10 in embryonic and postnatal development". In: Proceedings of the National Academy of Sciences 110.29 (2013), pp. 11887-11892. DOI: 10.1073/pnas . 1306074110. 
[17] Jang Hwan Cho, James J Collins, and Wilson W Wong. "Universal Chimeric Antigen Receptors for Multiplexed and Logical Control of T Cell Responses". en. In: Cell 173.6 (May 2018), 1426-1438.e11. ISSN: 0092-8674, 1097-4172. DoI: $10.1016 / j . c e l l .2018$. Q3.038.

[18] Hans Clevers. "The intestinal crypt, a prototype stem cell compartment". en. In: Cell 154.2 (July 2013), pp. 274-284. ISSN: 0092-8674, 1097-4172. Dor: 10.1016/j . cell.2013.07. 004 .

[19] Michael Costanzo et al. "A global genetic interaction network maps a wiring diagram of cellular function”. en. In: Science 353.6306 (Sept. 2016). ISSN: 0036-8075, 1095-9203. DOI: 10.1126/science.aaf1420.

[20] G M Edelman and J A Gally. "Degeneracy and complexity in biological systems". en. In: Proc. Natl. Acad. Sci. U. S. A. 98.24 (Nov. 2001), pp. 13763-13768. ISSN: 0027-8424. DOI: 10.1073/pnas.231499798.

[21] Marie Eubelen et al. "A molecular mechanism for Wnt ligand-specific signaling”. en. In: Science 361.6403 (Aug. 2018). ISSN: 0036-8075, 1095-9203. DoI: $10.1126 /$ science. aat1178.

[22] Chiara Francavilla et al. "Multilayered proteomics reveals molecular switches dictating ligand-dependent EGFR trafficking”. en. In: Nat. Struct. Mol. Biol. 23.6 (June 2016), pp. 608618. ISSN: 1545-9993, 1545-9985. DOI: 10.1038/nsmb.3218.

[23] Daniel M Freed et al. "EGFR Ligands Differentially Stabilize Receptor Dimers to Specify Signaling Kinetics". en. In: Cell 171.3 (Oct. 2017), 683-695.e18. IsSN: 0092-8674, 10974172. DoI: $10.1016 / \mathrm{j}$.cell.2017.09.017.

[24] Gaoxiang Ge and Daniel S Greenspan. "BMP1 controls TGF $\beta 1$ activation via cleavage of latent TGF $\beta$-binding protein". In: J. Cell Biol. 175.1 (Oct. 2006), pp. 111-120. ISSN: 0021-9525. DOI: $10.1083 / \mathrm{jcb} .200606058$.

[25] R E Godin, E J Robertson, and A T Dudley. "Role of BMP family members during kidney development”. en. In: Int. J. Dev. Biol. 43.5 (1999), pp. 405-411. ISSN: 0214-6282.

[26] Pauline Gonnord et al. "A hierarchy of affinities between cytokine receptors and the common gamma chain leads to pathway cross-talk". en. In: Sci. Signal. 11.524 (Apr. 2018). ISSN: 1937-9145, 1945-0877. DoI: 10.1126/scisignal.aal1253

[27] Hannah Greenfeld, Jerome Lin, and Mary C Mullins. "The BMP signaling gradient is interpreted through concentration thresholds in dorsal-ventral axial patterning". In: PLoS Biol. 19.1 (Jan. 2021), e3001059. IsSN: 1544-9173, 1545-7885. DoI: 10.1371/journal. pbio.3001059.

[28] Chul Han et al. "SMAD1 deficiency in either endothelial or smooth muscle cells can predispose mice to pulmonary hypertension". en. In: Hypertension 61.5 (May 2013), pp. 10441052. ISSN: 0194-911X, 1524-4563. DOI: 10.1161/HYPERTENSIONAHA.111.199158.

[29] Raymond C Harris, Eunkyung Chung, and Robert J Coffey. "EGF receptor ligands". en. In: Exp. Cell Res. 284.1 (Mar. 2003), pp. 2-13. ISSN: 0014-4827. DoI: 10.1016/s00144827(02)00105-2. 
[30] Anke Hartung et al. "Different routes of bone morphogenic protein (BMP) receptor endocytosis influence BMP signaling”. en. In: Mol. Cell. Biol. 26.20 (Oct. 2006), pp. 7791-7805. ISSN: 0270-7306. DOI: 10.1128/MCB.00022-06.

[31] Kai Heinecke et al. "Receptor oligomerization and beyond: a case study in bone morphogenetic proteins". en. In: BMC Biol. 7 (Sept. 2009), p. 59. ISSN: 1741-7007. DoI: 10.1186/ 1741-7007-7-59.

[32] P Heitzler and P Simpson. "The choice of cell fate in the epidermis of Drosophila". en. In: Cell 64.6 (Mar. 1991), pp. 1083-1092. ISSN: 0092-8674. DoI: 10.1016/0092-8674(91)90263$\mathrm{X}$.

[33] Christian Hiepen et al. "BMPR2 acts as a gatekeeper to protect endothelial cells from increased TGF $\beta$ responses and altered cell mechanics". en. In: PLoS Biol. 17.12 (Dec. 2019), e3000557. ISSN: 1544-9173, 1545-7885. DoI: 10.1371/journal .pbio. 3000557.

[34] Michael J Isaacs et al. "Bone morphogenetic protein-2 and -6 heterodimer illustrates the nature of ligand-receptor assembly”. en. In: Mol. Endocrinol. 24.7 (July 2010), pp. 14691477. ISSN: 0888-8809, 1944-9917. DOI: 10.1210/me.2009-0496.

[35] Simon Joost et al. "The Molecular Anatomy of Mouse Skin during Hair Growth and Rest". en. In: Cell Stem Cell 26.3 (Mar. 2020), 441-457.e7. ISSN: 1934-5909, 1875-9777. DOI: $10.1016 /$ j.stem.2020.01.012

[36] E Kessler et al. "Bone morphogenetic protein-1: the type I procollagen C-proteinase". en. In: Science 271.5247 (Jan. 1996), pp. 360-362. ISSN: 0036-8075. DOI: 10.1126/science. 271.5247 .360 .

[37] Najin Kim et al. "BMP-dependent synaptic development requires Abi-Abl-Rac signaling of BMP receptor macropinocytosis". en. In: Nat. Commun. 10.1 (Feb. 2019), p. 684. ISSN: 2041-1723. DOI: 10.1038/s41467-019-08533-2.

[38] Raphael Kopan and Maria Xenia G Ilagan. "The canonical Notch signaling pathway: unfolding the activation mechanism". en. In: Cell 137.2 (Apr. 2009), pp. 216-233. ISSN: 0092-8674, 1097-4172. DOI: $10.1016 / \mathrm{j}$.cell.2009.03.045.

[39] C C Kopczynski et al. "Delta, a Drosophila neurogenic gene, is transcriptionally complex and encodes a protein related to blood coagulation factors and epidermal growth factor of vertebrates". en. In: Genes Dev. 2.12B (Dec. 1988), pp. 1723-1735. ISSN: 0890-9369. DOI: 10.1101/gad.2.12b.1723.

[40] François Lapraz, Lydia Besnardeau, and Thierry Lepage. "Patterning of the dorsal-ventral axis in echinoderms: insights into the evolution of the BMP-chordin signaling network". en. In: PLoS Biol. 7.11 (Nov. 2009), e1000248. ISSN: 1544-9173, 1545-7885. DOI: $10.1371 /$ journal.pbio. 1000248 .

[41] Lauren LeBon et al. "Fringe proteins modulate Notch-ligand cis and trans interactions to specify signaling states". en. In: Elife 3 (Sept. 2014), e02950. ISSN: 2050-084X. DOI: 10.7554/eLife.02950. 
[42] Ruth Lehmann et al. "On the phenotype and development of mutants of early neurogenesis inDrosophila melanogaster”. en. In: Wilehm Roux Arch Dev Biol 192.2 (Mar. 1983), pp. 6274. ISSN: 0340-0794. DOI: $10.1007 /$ BF00848482.

[43] Vincent C Luca et al. "Notch-Jagged complex structure implicates a catch bond in tuning ligand sensitivity”. en. In: Science 355.6331 (Mar. 2017), pp. 1320-1324. ISSN: 0036-8075, 1095-9203. DOI: 10.1126/science . aaf9739.

[44] Vincent C Luca et al. "Structural basis for Notch1 engagement of Delta-like 4". en. In: Science 347.6224 (Feb. 2015), pp. 847-853. ISSN: 0036-8075, 1095-9203. DOI: 10.1126/ science. 1261093 .

[45] Philippe Lucarelli et al. "Resolving the combinatorial complexity of smad protein complex formation and its link to gene expression". In: Cell systems 6.1 (2018), pp. 75-89.

[46] Joan Massagué. “TGF $\beta$ signalling in context”. In: Nature reviews Molecular cell biology 13.10 (2012), pp. 616-630.

[47] David W McCauley and Marianne Bronner-Fraser. "Conservation and divergence of BMP2/4 genes in the lamprey: expression and phylogenetic analysis suggest a single ancestral vertebrate gene". en. In: Evol. Dev. 6.6 (Nov. 2004), pp. 411-422. ISSN: 1520-541X. DOI: $10.1111 / \mathrm{j} .1525-142 \mathrm{X} .2004 .04054 . \mathrm{x}$

[48] T H Morgan. "The theory of the gene". In: Am. Nat. 51.609 (Sept. 1917), pp. 513-544. ISSN: 0003-0147, 1537-5323. DOI: 10.1086/279629.

[49] Leonardo Morsut et al. "Engineering Customized Cell Sensing and Response Behaviors Using Synthetic Notch Receptors”. en. In: Cell 164.4 (Feb. 2016), pp. 780-791. ISSN: 00928674, 1097-4172. DoI: 10.1016/j.cell.2016.01.012.

[50] Thomas D Mueller and Joachim Nickel. "Promiscuity and specificity in BMP receptor activation”. In: FEBS letters 586.14 (2012), pp. 1846-1859.

[51] Nagarajan Nandagopal et al. "Dynamic Ligand Discrimination in the Notch Signaling Pathway”. en. In: Cell 172.4 (Feb. 2018), 869-880.e19. ISSN: 0092-8674, 1097-4172. DOI: $10.1016 / \mathrm{j} . \mathrm{cell} .2018 .01 .002$

[52] NASA Press Conference Transcript February 22: Perseverance Rover Searches for Life on Mars. https : / / wWW . rev . com/blog/transcripts / nasa-press - conference transcript-february-22-perseverance-rover-searches-for-life-on-mars. Accessed: 2021-3-18. Feb. 2021.

[53] Joachim Nickel and Thomas D Mueller. "Specification of BMP Signaling". en. In: Cells 8.12 (Dec. 2019). ISSN: 2073-4409. DOI: 10.3390/cells8121579.

[54] R Nusse and H E Varmus. "Wnt genes". en. In: Cell 69.7 (June 1992), pp. 1073-1087. ISSN: 0092-8674. DOI: 10.1016/0092-8674(92)90630-u.

[55] Amanda Ochoa-Espinosa and Markus Affolter. "Branching morphogenesis: from cells to organs and back”. en. In: Cold Spring Harb. Perspect. Biol. 4.10 (Oct. 2012). ISSN: 19430264. DOI: 10.1101/cshperspect.a008243. 
[56] David M Ornitz and Nobuyuki Itoh. The Fibroblast Growth Factor signaling pathway. 2015. DOI: $10.1002 / \mathrm{wdev} .176$.

[57] Mar Orriols, Maria Catalina Gomez-Puerto, and Peter Ten Dijke. "BMP type II receptor as a therapeutic target in pulmonary arterial hypertension". en. In: Cell. Mol. Life Sci. 74.16 (Aug. 2017), pp. 2979-2995. ISSN: 1420-682X, 1420-9071. Dor: 10.1007/s00018-017-2510-4.

[58] Leif Oxburgh et al. "BMP4 substitutes for loss of BMP7 during kidney development". en. In: Dev. Biol. 286.2 (Oct. 2005), pp. 637-646. ISSN: 0012-1606. DoI: $10.1016 / \mathrm{j}$. ydbio. 2005.08 .024

[59] E Ozkaynak et al. "OP-1 cDNA encodes an osteogenic protein in the TGF-beta family". en. In: EMBO J. 9.7 (July 1990), pp. 2085-2093. ISSN: 0261-4189, 1460-2075. DOI: 10.1002/ j.1460-2075.1990.tb07376.x.

[60] E Ozkaynak et al. "Osteogenic protein-2. A new member of the transforming growth factorbeta superfamily expressed early in embryogenesis". en. In: J. Biol. Chem. 267.35 (Dec. 1992), pp. 25220-25227. ISSN: 0021-9258.

[61] Orhan Özüak et al. "Ancient and diverged TGF- $\beta$ signaling components in Nasonia vitripennis". In: Development Genes and Evolution 224.4-6 (2014), pp. 223-233. Dor: 10 . $1007 /$ s00427-014-0481-0

[62] Jillian Rosenberg et al. "Lattice Light-Sheet Microscopy Multi-dimensional Analyses (LaMDA) of T-Cell Receptor Dynamics Predict T-Cell Signaling States". In: Cell Systems 10.5 (2020), 433-444.e5. Dor: $10.1016 / \mathrm{j}$.cels.2020.04.006.

[63] Kole T Roybal et al. "Precision Tumor Recognition by T Cells With Combinatorial AntigenSensing Circuits”. en. In: Cell 164.4 (Feb. 2016), pp. 770-779. IsSN: 0092-8674, 1097-4172. Dor: $10.1016 / \mathrm{j} . \mathrm{cell} .2016 .01 .011$.

[64] Valerie S Salazar, Laura W Gamer, and Vicki Rosen. "BMP signalling in skeletal development, disease and repair". en. In: Nat. Rev. Endocrinol. 12.4 (Apr. 2016), pp. 203-221. ISSN: 1759-5029, 1759-5037. DoI: $10.1038 /$ nrendo.2016.12.

[65] T K Sampath, N Muthukumaran, and A H Reddi. "Isolation of osteogenin, an extracellular matrix-associated, bone-inductive protein, by heparin affinity chromatography". en. In: Proc. Natl. Acad. Sci. U. S. A. 84.20 (Oct. 1987), pp. 7109-7113. ISSN: 0027-8424. DoI:10.1073/ pnas.84.20.7109

[66] T K Sampath and A H Reddi. "Dissociative extraction and reconstitution of extracellular matrix components involved in local bone differentiation". en. In: Proc. Natl. Acad. Sci. U. S. A. 78.12 (Dec. 1981), pp. 7599-7603. ISSN: 0027-8424. Dor: 10.1073/pnas.78.12.7599.

[67] Stefan Saremba et al. "Type I receptor binding of bone morphogenetic protein 6 is dependent on N-glycosylation of the ligand: Type I receptor specificity of BMP-6". en. In: FEBS J. 275.1 (Jan. 2008), pp. 172-183. ISSN: $1742-464 X, 1742-4658$. DOI: $10.1111 / \mathrm{j} .1742-$ $4658.2007 .06187 . \mathrm{x}$ 
[68] Monika Saxena, Nitin Agnihotri, and Jonaki Sen. "Perturbation of canonical and noncanonical BMP signaling affects migration, polarity and dendritogenesis of mouse cortical neurons". en. In: Development 145.1 (Jan. 2018). ISSN: 0950-1991, 1477-9129. DOI: 10. $1242 /$ dev. 147157

[69] E H Schroeter, J A Kisslinger, and R Kopan. "Notch-1 signalling requires ligand-induced proteolytic release of intracellular domain”. en. In: Nature 393.6683 (May 1998), pp. 382386. ISSN: 0028-0836. DOI: $10.1038 / 30756$.

[70] Howard J Seeherman et al. "A BMP/activin A chimera is superior to native BMPs and induces bone repair in nonhuman primates when delivered in a composite matrix". en. In: Sci. Transl. Med. 11.489 (Apr. 2019). ISSN: 1946-6234, 1946-6242. DOI: 10 . $1126 /$ scitranslmed.aar4953.

[71] M Shibuya. "Vascular Endothelial Growth Factor (VEGF) and Its Receptor (VEGFR) Signaling in Angiogenesis: A Crucial Target for Anti- and Pro-Angiogenic Therapies". In: Genes \& Cancer 2.12 (2011), pp. 1097-1105. DOI: 10.1177/1947601911423031.

[72] Elaine E Storm et al. "Limb alterations in brachypodism mice due to mutations in a new member of the TGF $\beta$-superfamily”. en. In: Nature 368.6472 (Apr. 1994), pp. 639-643. ISSN: 0028-0836. DOI: $10.1038 / 368639 a$.

[73] Christina J Su et al. "Ligand-receptor promiscuity enables cellular addressing". In: In preparation (2020).

[74] M R Urist. "Bone: formation by autoinduction". en. In: Science 150.3698 (Nov. 1965), pp. 893-899. ISSN: 0036-8075. DOI: 10.1126/science.150.3698.893.

[75] Marshall R Urist and Basil S Strates. "Bone Morphogenetic Protein". In: Journal of Dental Research 50.6 (1971), pp. 1392-1406. DOI: 10.1177/00220345710500060601.

[76] Zara Y. Weinberg et al. "Sentinel cells enable genetic detection of SARS-CoV-2 Spike protein". In: bioRxiv (2021). DOI: 10.1101/2021.04.20.440678, eprint: https: //wWW . biorxiv.org/content/early/2021/04/20/2021.04.20.440678. full .pdf. URL: https://wWw.biorxiv.org/content/early/2021/04/20/2021.04.20.440678.

[77] Denise Wootten et al. "Mechanisms of signalling and biased agonism in G protein-coupled receptors". en. In: Nat. Rev. Mol. Cell Biol. 19.10 (Oct. 2018), pp. 638-653. ISSN: 1471-0072, 1471-0080. DOI: $10.1038 / \mathrm{s} 41580-018-0049-3$.

[78] J M Wozney et al. "Novel regulators of bone formation: molecular clones and activities". en. In: Science 242.4885 (Dec. 1988), pp. 1528-1534. ISSN: 0036-8075. DOI: 10. $1126 /$ science. 3201241 .

[79] Pamela Yeh, Ariane I Tschumi, and Roy Kishony. "Functional classification of drugs by properties of their pairwise interactions". en. In: Nat. Genet. 38.4 (Apr. 2006), pp. 489-494. ISSN: 1061-4036. DOI: $10.1038 / \mathrm{ng} 1755$.

[80] Atilgan Yilmaz et al. "MuSK is a BMP co-receptor that shapes BMP responses and calcium signaling in muscle cells”. en. In: Sci. Signal. 9.444 (Sept. 2016), ra87. ISSN: 1937-9145, 1945-0877. DOI: 10.1126/scisignal . aaf0890 
Chapter 2

\section{COMBINATORIAL, CONTEXT-DEPENDENT LOGIC OF BMP SIGNALING}

\subsection{Summary}

Cell-cell communication systems typically comprise families of ligand and receptor variants that function together in combinations. Pathway activation depends in a complex way on which ligands are present and what receptors are expressed by the signal-receiving cell. To understand the combinatorial logic of such a system, we systematically measured pairwise Bone Morphogenetic Protein (BMP) ligand interactions in cells with varying receptor expression. Ligands could be classified into equivalence groups based on their profile of positive and negative synergies with other ligands. These groups varied with receptor expression, explaining how ligands can functionally replace each other in one context but not another. Context-dependent combinatorial interactions could be explained by a biochemical model based on competitive formation of alternative signaling complexes with distinct activities. Together, these results provide insights into the roles of BMP combinations in developmental and therapeutic contexts and establish a framework for analyzing other combinatorial, context-dependent signaling systems.

Keywords: bone morphogenetic protein, BMP, signaling pathways, cell context, combinatorial signaling, promiscuous receptor-ligand interactions, pairwise interaction analysis

\subsection{Introduction}

Cell communication pathways such as BMP, Wnt, and FGF play pivotal roles in normal development, disease, therapeutics, and regenerative medicine. $\frac{57 / 46 / 48}{A}$ striking feature of these pathways is their use of families of homologous ligand variants. Within each pathway, multiple ligands typically function together in combinations, ${ }^{4235}$ and the effect of any given ligand can vary dramatically with cell or tissue context..$^{15[36 / 74}$ Despite the prevalence of these features, and extensive information about the biochemical interactions and developmental roles of particular ligands, we lack a unified description of how ligands signal in combinations and across biological contexts. A clearer view of this structure could allow more predictive control of these pathways in natural and synthetic contexts.

A prime example of such combinatorial and contextual ligand activity can be seen in the Bone Morphogenetic Protein (BMP) pathway. This pathway comprises ten major ligand variants, as well as four Type I and three Type II receptors subunits (Figure 2.1A). Distinct combinations 
of these components regulate the development of diverse tissues including skeleton, kidney, eye, and brain. $57 / 25|33| 12$ Interestingly, a pair of ligands can exhibit either redundant or distinct roles depending on context. For example, BMP9 can replace the essential role of BMP10 in proper vasculature formation, but not in the developing heart. ${ }^{15}$ Similarly, BMP4 can replace BMP7 in the developing kidney, but not in the developing eye ${ }^{50}$ In addition to variation across tissue context, BMP ligands also have non-overlapping roles when signaling in combinations, such as observed in the developing joint, ${ }^{7]}$ and can combine with each other non-additively $\left[\frac{54 / 79 / 38 / 4}{4}\right.$

Thus far, the primary framework for describing BMP ligand differences is variation in ligandreceptor affinity. Ligand-receptor affinities govern the formation of signaling complexes, each of which contains a dimeric ligand bound to two Type I and two Type II receptor subunits (Figure 2.1A). These signaling complexes phosphorylate SMAD1/5/8 effector proteins (as well as activate other non-canonical targets) to regulate target genes. Ligands are often grouped by their preferred Type I and Type II receptors to summarize their functional differences $\frac{6421}{21}$ However, receptor preferences cannot explain key functional differences between ligands. For instance BMP9 and BMP10 exhibit similar receptor preferences ${ }^{22}$ but behave non-equivalently, as mentioned above..$^{15}$ Moreover, BMP receptors are expressed in combinations (Figure 2.1B), compete with one another for binding to ligands, and generate complex functional responses to ligand combinations, all of which make it difficult to predict the distribution of signaling complexes, and thereby the overall pathway activity, from qualitative affinity preferences alone. ${ }^{4}$ Therefore, despite extensive molecular characterization, we cannot in general say how specific ligands will function in combinations, why these combinatorial effects vary with context, and what molecular features are necessary to generate the combinatorial and contextual behavior of BMP ligands. Addressing these questions would advance our understanding of the role of BMP signaling components in a variety of skeletal, respiratory, and brain diseases $\frac{57 / 72 / 19}{}$ and aid the selection of therapeutic BMPs to selectively induce bone growth in orthopedics and oral surgery. $\frac{16 / 1361}{16}$ More generally, it could enable more precise control of cell fate decisions for therapeutic applications and provide insight into the overall logic of cell-cell communication systems.

To obtain a systematic understanding of context-dependent ligand integration, we turned to pairwise interaction analysis, an approach that has provided powerful insights into the structure of other combinatorial biological systems. In this approach, one classifies pairs of perturbations as additive (neutral), positive (synergistic), or negative (antagonistic) depending on whether the perturbations are stronger or weaker in combination than expected from their individual effects. Pairwise analysis of mutations has revealed the structure of gene modules and protein functions. $60|62| 17$ Similarly, pairwise analysis of drug interactions successfully classified antibiotics into groups with similar biochemical mechanisms of action. ${ }^{78}$ We reasoned that pairwise analysis of BMP 
A

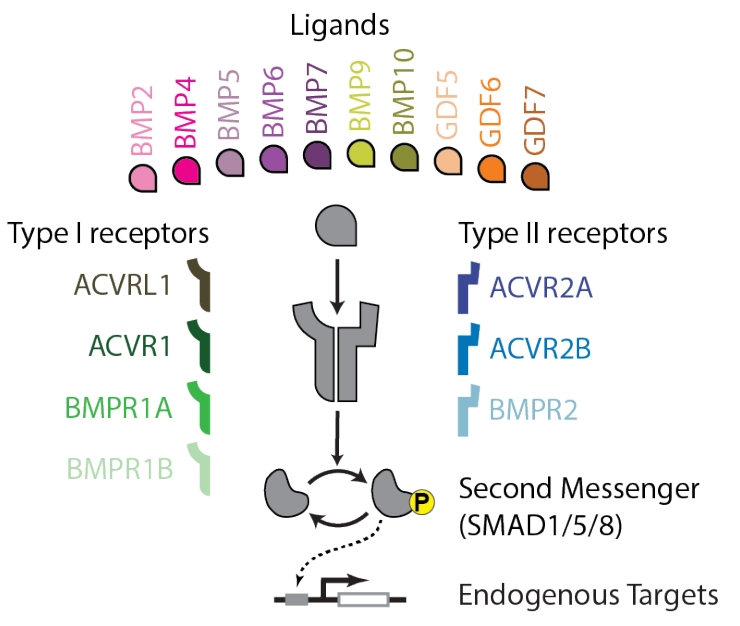

B

B Combinatorial expression

of pathway components
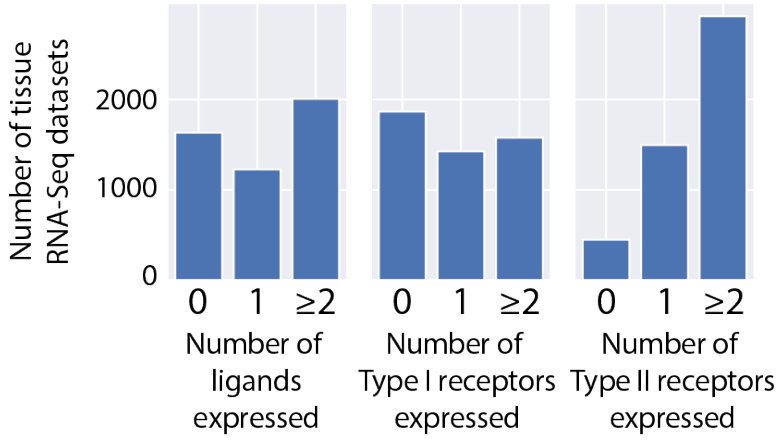

C

Characterization

Individual ligand strengths

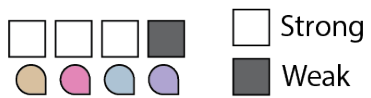

Pairwise Interactions

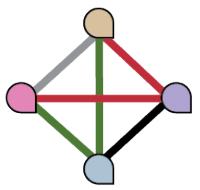

Synergistic

Additive

Antagonistic

Suppressive

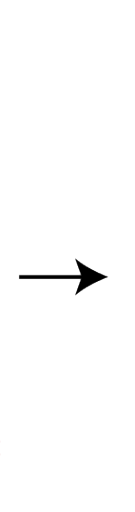

Clustering

Individual strength

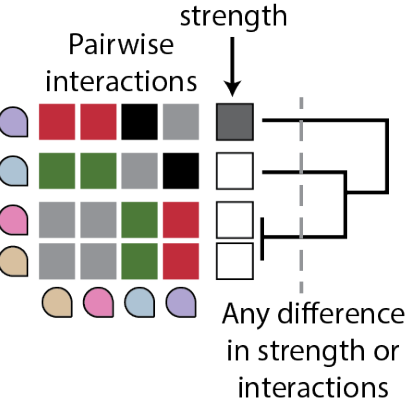

Equivalence groups

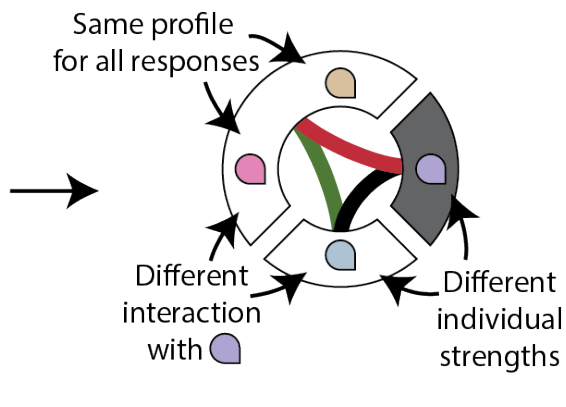

Figure 2.1: BMP ligands can be classified by their pairwise interactions.

(A) BMP ligands (colored petal shapes) bind Type I and Type II receptors to form signaling complexes. Active signaling complexes phosphorylate SMAD1/5/8 transcription factors, which translocate to the nucleus to activate endogenous gene targets. Promiscuous ligand-receptor interactions enable the formation of a wide variety of potential signaling complexes. The dimeric nature of the ligands allows each to recruit up to two Type I and two Type II receptors simultaneously.

(B) Bulk RNA-Seq datasets of various mouse tissues, collected in [40], show that BMP ligands, Type I receptors, and Type II receptors are often expressed (i.e. FPKM > 0) as combinations (i.e. 2 or more).

(C) Systematic measurements of individual and pairwise responses (left) can cluster BMP ligands into equivalence groups (middle). Ligands in the same equivalence group interact in similar ways (synergistic, additive, antagonistic, or suppressive) with other ligands and have the same individual strength (right).

ligand combinations could be useful in two ways: First, it could provide a systematic map of how ligands combine to determine pathway activation (Figure 2.1C). Second, it could reveal how that combinatorial structure itself changes between different cell contexts. In this way, pairwise analysis of ligand combinations could provide a general way to understand systems that are both combinatorial and contextual. With that in mind, we analyzed pairwise combinations of ten of the 
most important BMP ligands across multiple receptor contexts. (Analogous to the convention for drug interactions, we will use the term "ligand interactions" to refer to non-additive responses to ligand combinations, without implying direct molecular interaction between the ligands.) We first show how this pairwise analysis classifies BMP ligands into equivalence groups, defined such that ligands in the same group exhibit a similar strength and profile of effective interactions with other ligands (Figure 2.1C). This analysis reveals that the ten ligands can be organized into a smaller number of equivalence groups, and introduces a compact, useful representation for complex multiligand systems. (Ligand equivalence groups, defined here, are distinct from equivalence groups in development, which describe groups of cells with the same fate potential. ${ }^{[27}$ ) Next, we turn to the question of how ligand interactions vary with cell context, measuring ligand interactions using cells with differing receptor expression profiles. We find that receptor expression plays a powerful, non-intuitive role in shaping combinatorial ligand responses, and that receptor context effects can explain previous observations in development. Finally, to understand how the observed combinatorial ligand responses could emerge from underlying molecular features of the pathway, we fit the data to a mathematical model of competitive ligand-receptor interactions. We find that contextual multi-ligand BMP responses can emerge from the interplay between the affinities that govern signaling complex formation and the specific phosphorylation activities of the resulting complexes. Together, these results reveal the combinatorial and contextual logic of BMP signaling, and provide a framework for understanding other pathways that similarly integrate multi-ligand inputs with promiscuous protein-protein interactions.

\subsection{Results}

\subsubsection{Quantitative dose-response measurements reveal ligand-specific features.}

Analyzing ligand combinations first requires an understanding of how the BMP pathway responds to ligands individually. To that end, we identified a core set of BMP ligands for our study from the larger set of TGF- $\beta$ superfamily ligands, ${ }^{76}$ focusing on ten homodimeric BMP ligands that play pivotal roles in development and are known to activate SMAD1/5/8 (Figure 2.1 A) ${ }^{32}$ Next, we constructed a BMP reporter cell line (Figure S2.1A). As a base cell line, we selected NAMRU mouse mammary gland (NMuMG) epithelial cells, which can respond to BMP signals without differentiating 5 and expresses five of the seven BMP receptors, including ACVR1, ACVR2A, ACVR2B, BMPR1A, and BMPR $2{ }^{4}$ We integrated a construct encoding a fusion Histone $2 \mathrm{~B}(\mathrm{H} 2 \mathrm{~B})$-mCitrine protein controlled by a synthetic SMAD1/5/8-activated BMP responsive element (BRE). ${ }^{39}$ The resulting stable cell line exhibited unimodal, dose-dependent distributions of YFP fluorescence levels in response to varying concentrations of BMP ligands (Figure $\mathrm{S2.1} B$ ), allowing quantitative readout of pathway activity and ligand dose responses (Figure $\mathbf{S 2 . 1}$ C). 
The ligands exhibited a broad range of dose-response characteristics (Figure S2.1D). Seven ligands strongly activated the pathway but did so with varying saturating levels, concentrations for halfmaximal activation $\left(\mathrm{EC}_{50}\right.$ ), and logarithmic sensitivities, quantified by a fitted Hill coefficient. The $\mathrm{EC}_{50}$ ranged over three orders of magnitude among these ligands (Figure $\mathrm{S} 2.1 \mathrm{E}$ ) while the Hill coefficient varied between values of one and two (Figure $\mathrm{S} 2.1 \mathrm{~F}$ ). By contrast, the Relative Ligand Strength (RLS), or the pathway activity at saturating concentrations normalized to the activity of the strongest ligand, could be quantified for all 10 ligands and fell into three distinct tiers (Figure S2.1G). In the lowest tier, GDF5, GDF6, and GDF7 barely activated the reporter above background. However, these ligands did activate the pathway following ectopic expression of BMPR1B (Figure $\mathrm{S} 2.6 \mathrm{E})$, a receptor not expressed by NMuMG cells and required for GDF signaling. 45

\subsubsection{Ligands exhibit diverse combinatorial profiles.}

While the single-ligand dose responses identified ligands of equivalent strength (quantified here as RLS), they were not sufficient to classify ligand equivalence in combinations. To systematically probe combinatorial responses, we measured the responses to all 45 pairs of the 10 ligands (Figure $2.2 \mathrm{~A}$ ), as well as each ligand alone or paired with itself. The interactions of each ligand pair were observed at nine distinct ratios, using concentrations spanning the ligand's dynamic range (Figure 2.2A, inset). A robotic liquid-handling system enabled measurements of multiple ligand combinations in a single experiment (STAR Methods), and responses were quantified by flow cytometry after $24 \mathrm{~h}$ incubation with the ligands (Figures $2.2 \mathrm{~B}-\mathrm{E}$ ). 
A

Screen set-up

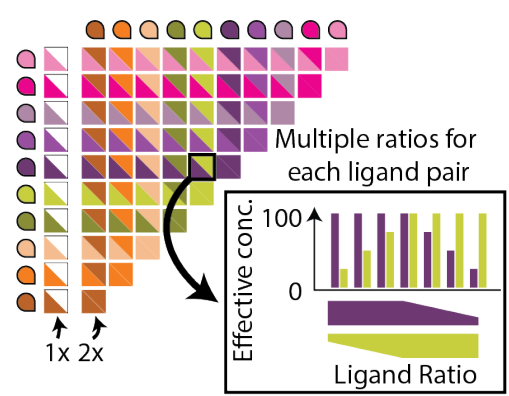

C

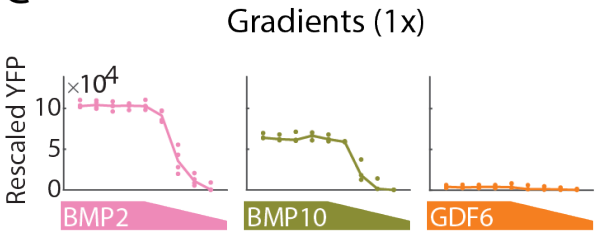

D

Pair with self (2x)
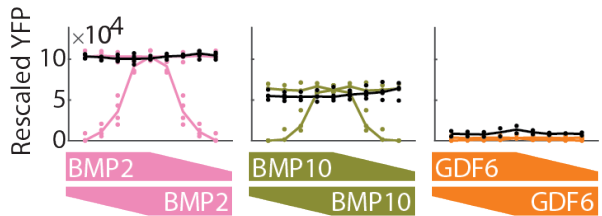

E

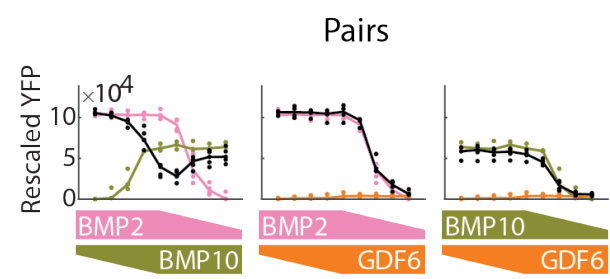

Rescaled YFP Median

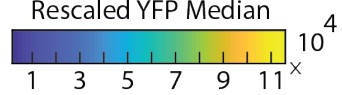

B

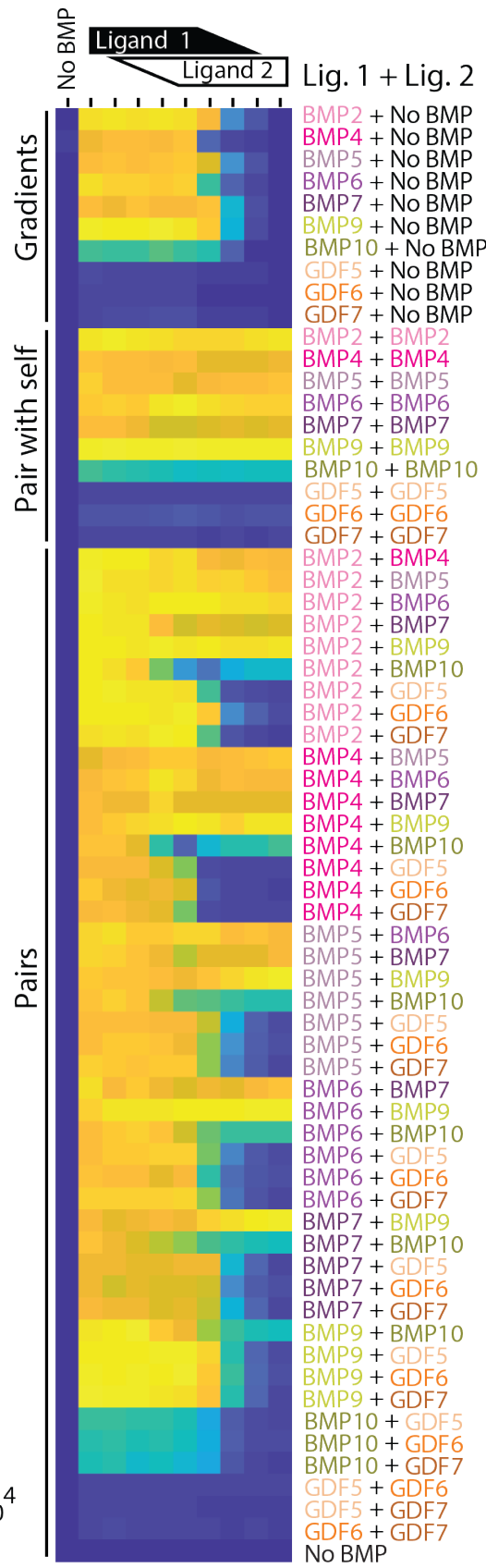

F
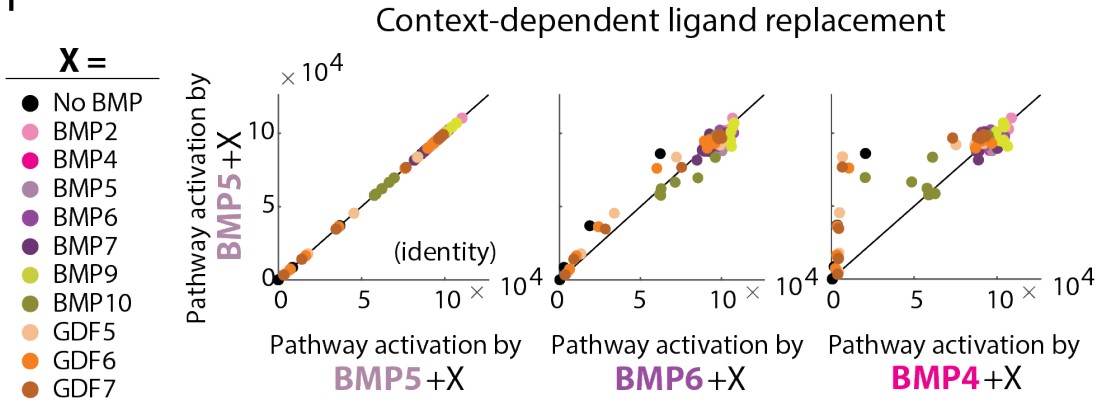

Pathway activation by Pathway activation by BMP6+X BMP4+X

Figure 2.2: Pairwise titration of relative ligand concentrations reveals ligand interactions. (caption on following page) 
Figure 2.2 (previous page):

(A) Fully classifying ligand equivalence groups requires analysis of all possible pairs, as well as measurements of individual activity (1X) and each ligand paired with itself (2X) as a positive control. Responses in each category are sampled at multiple ratios (inset), with at least one ligand very near its saturating concentration in each ratio.

(B) The full dataset includes measurements for all possible gradients, pairs, and pairs with self, which are shown as each row of the heatmap. The columns correspond to concentration ratios, with the leftmost column reserved for a no BMP control. Medians of four biological repeats are shown with heatmap colors.

(C) Sample gradients of BMP2, BMP10, and GDF6 show diversity of individual strength. Dots are four biological replicates, and the line connects the median value for each concentration. The ligand concentrations are high and saturating on the left, and decrease from left to right, as shown by the trapezoid height on the $\mathrm{x}$-axis.

(D) Ligands paired with themselves show purely additive relationships between ligands of the same strength. Colored dots and lines summarize biological replicates and median for the ligand of the indicated color, whereas the black line shows the pair indicated on the x-axis, either BMP2, BMP10, or GDF6 paired with itself for multiple concentration ratios.

(E) All possible pairs of three ligands (BMP2, BMP10, and GDF6) show three types of pairwise interactions. Responses to pairs can be lower than both individual ligands (left), exactly equal to stronger of the two (middle), or interpolate between the two (right). As in $\mathrm{C}$, the colored dots and lines show individual ligand responses, whereas black is the response to the pair. The concentration ratios are shown using the same trapezoids on the $\mathrm{x}$-axis.

(F) Responses to BMP5 paired with another ligand ("X", colors) are plotted against the response to other ligands (BMP5, BMP6, or BMP7) also paired with X. Since all pairs are sampled at seven ratios, there are seven dots for each $\mathrm{X}$ (i.e. color). The first two plots show that BMP6 is a nearly equivalent replacement for BMP5, as BMP5+X closely resembles BMP6+X (i.e. dots are close to the $\mathrm{y}=\mathrm{x}$ line). By contrast, BMP4 produces much lower activation than an equivalent amount of BMP5, in the presence of BMP10, GDF5, GDF6, or GDF7. Pathway activation is the median of four biological repeats, quantified by flow cytometry.

Comparing two ligand's activities across combinatorial conditions summarizes their ability to produce equivalent pathway output (quantified here with YFP fluorescence) in the presence of another ligand. For example, consider BMP4, BMP5, and BMP6, which are all strong activators with RLS close to 1. By definition, replacing BMP5 with itself produces equivalent responses across all conditions (Figure 2.2F, left). Replacing BMP5 with BMP6 also produced very similar pathway activity, in the context of any other ligand or ratio, indicating that these two ligands behave equivalently in this context (Figure 2.2F, middle). By contrast, replacing BMP5 with an equivalent dose of BMP4 changed signaling activity in the presence of BMP10 or any of the GDFs (Figure $2.2 F$, right). Thus, ligands that exhibit similar RLS (strength) are not necessarily equivalent, due to the differences in their pairwise interactions with other ligands. 


\subsubsection{Ligand interactions reveal BMP equivalence groups in NMuMG cells.}

Because differences in pairwise interactions are key to determining ligand equivalence, we sought a metric to quantitatively classify the type and strength of ligand interactions. Common drug interaction metrics, such as Loewe or Bliss, and epistasis metrics do not precisely map onto ligand interactions, ${ }^{41|9| 56 / 78}$ so we defined the Interaction Coefficient (IC) (Figures 2.3 A, S2.2A, STAR Methods). The IC is designed to map qualitatively different interaction types and magnitudes onto a single linear scale by comparing the combined and individual effects of two ligands. This scale separates qualitatively different behaviors into different IC regimes and includes both linear $(\mathrm{IC}=1)$ and saturated ( $\mathrm{IC}=0$ ) additivity, as well as negative interactions ( $\mathrm{IC}<0)$ and positive synergy (IC $>1)$. The resulting scale can be written as a set of piecewise-linear equations (Figure S2.2A), which correctly classify distinct behaviors (Figure $\mathrm{S} 2.2 \mathrm{~B}$ ).
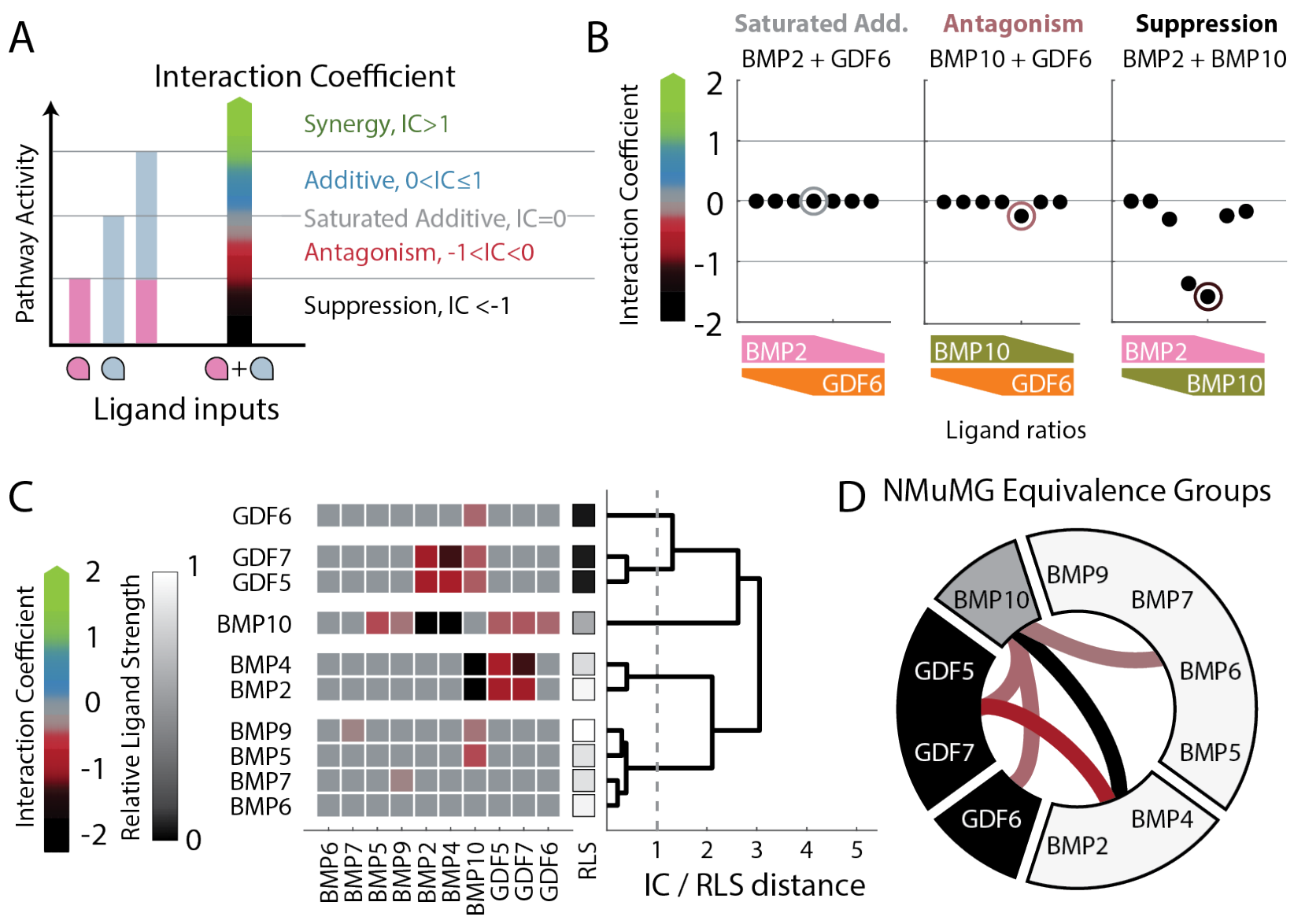

Figure 2.3: Antagonism and suppression define BMP equivalence groups in NMuMG cells. (caption on following page) 
Figure 2.3 (previous page):

(A) The Interaction Coefficient (IC) classifies different regimes of pairwise responses by comparison to three references (horizontal lines): the two individual ligand responses, $f(A)$ and $f(B)$, and the sum of those responses, $f(A)+f(B)$. The behavior type and strength for a given pairwise response, $f(A+B)$, is indicated by its IC value and associated color map: black for suppressive, red for antagonistic, gray for saturated additive, blue for additive, and green for synergistic. IC values linearly interpolate between reference points. Though IC has no upper bound, values rarely exceed 2, which is the upper limit shown on all plots. See Figure $\mathrm{S} 2.1 \mathrm{~B}$ and Section 2.7 for formula.

(B) IC values for pairs of BMP2, BMP10, and GDF6 (as in Figure 2.2E) vary across pairs and multiple concentration ratios. Each pair exhibits a distinct response type. Circled IC values indicate which ratio was used to classify the pair, and the circle's color indicates the interaction type. Trapezoids on $\mathrm{x}$-axis indicate concentration ratios, analogous to inset in A.

(C) Hierarchical clustering of IC and RLS values in NMuMG cells, computed from the medians of four biological replicates, reveals five distinct equivalence groups, defined at a distance of 1 to separate ligands with similar pairwise and individual strengths (Section 2.7). The square colors indicate either the ligand's IC value (see colormap in B) when paired with another ligand or the RLS grayscale for its individual strength, as indicated by x-tick labels. The dendrogram on the right-hand side shows complete-linkage clustering of Euclidean distance between ligand features. The RLS and IC values are weighted such that a distance of 1 indicates a significant difference in either individual strength or a difference of one pairwise interaction between two ligands (Section 2.7).

(D) BMP ligand equivalence in NMuMG cells, as classified by hierarchical clustering in E, comprises five equivalence groups. Wedge grayscale value shows approximate RLS value of the contained ligands, while connecting line colors represent pairwise interactions between ligand groups. Groups without a linking edge interact additively. Ligands with similar individual strengths can be grouped differently based on their pairwise interactions.

See also Figures $\mathrm{S} 2.1, \mathrm{~S} 2.2$

Despite its different definition, IC's basic classifications mirror those of drug interactions, such as suppressive interactions. $\frac{78 \mid 11}{}$ These behaviors are also related to previously observed twoligand response types, with balance, additive, ratiometric, and imbalance functions producing synergistic, additive, antagonistic, and suppressive interaction coefficients, respectively. ${ }^{4}$ Moreover, like other interaction metrics, the IC value depends on the specific ligand concentrations for which it is computed, and is close to 1 at small enough concentrations of both ligands. Therefore, to characterize a ligand pair, we select the IC value of largest absolute value over all sampled concentration ratios (Figure 2.3 B), as pairwise interactions are most evident at certain ligand ratios and with total concentrations close to saturation (Figure $\mathrm{S} 2.2 \mathrm{~B}$ ). However, due to possible undersampling of concentration ratios and the unknown dynamic ranges of weakly activating ligands, these values may be underestimates of the strength of non-additive interactions. 
Nonetheless, the interaction metric (IC) revealed an array of interaction types within NMuMG cells' pairwise responses (Figure $\mathrm{S} 2.2 \mathrm{C}$ ). Most pairwise interactions were saturated additive (gray), but some showed antagonism (red) or suppression (black) (Figures 2.3 $\mathrm{C}, \mathrm{S} 2.2 \mathrm{C}-\mathrm{F}$ ). Combining the full set of pairwise interactions with measurements of individual ligand strength enabled clustering of ligands based on their functional similarity (Figure $2.3 \mathrm{C}$, dendrogram). Ligands appearing in the same cluster (group) exhibit the same pattern of interactions with other ligands, suggesting they function interchangeably across pairwise ligand combinations. These groups can be represented compactly around a circle (Figure 2.3D), with the grayscale level of each group indicating the approximate individual activity of its ligands and internal lines representing the non-additive interactions between groups. These groups included [BMP2, BMP4], [BMP5, BMP6, BMP7, BMP9], [BMP10], [GDF5, GDF7], and [GDF6]. Here and below, square brackets denote sets of ligands that constitute an equivalence group. In NMuMG cells, the full set of interactions among 10 ligands reduces to a set of five non-additive interactions among five distinct ligand groups. Ligands in the first group, [BMP5, BMP6, BMP7, BMP9], activated strongly as individuals and interacted additively with one another and all other ligands, except for weak antagonism with BMP10. The second group, [BMP2, BMP4], also contained strong activators, but was distinguished by its susceptibility to antagonism by GDF5 and GDF7 and suppression by BMP10. The third and fourth groups, [GDF5, GDF7] and [GDF6], comprised non-activating ligands that respectively did or did not antagonize [BMP2, BMP4]. Finally, [BMP10] formed its own equivalence group based on its unique interactions and intermediate strength. Thus, most of the ligand distinctions represented here emerge from the interactions and could not have been inferred from signaling properties of the individual ligands.

This systematic, interaction-based classification of ligands provides a multi-ligand perspective for predicting the effects of ligand combinations expressed in biological processes. For example, BMP2, BMP4, and BMP7 are coexpressed in skeletal development, but different combinatorial knockouts generate distinct skeletal defects. ${ }^{71}$ These results implied a unique role for BMP7, consistent with its classification in a separate equivalence group from BMP2 and BMP4. However, the equivalence map determined in NMuMG cells is not necessarily universal to all cell types. BMP receptor expression is variable across cell types in developing tissues, ${ }^{20}$ and changes in receptor expression can significantly alter pairwise ligand interactions, ${ }^{4}$ Therefore, these results provoke the question of how equivalence groups vary with cell context in general and with receptor expression profiles more specifically. 


\subsubsection{Ligand equivalence depends on cell context.}

Mouse embryonic stem cells (mESCs) present an ideal cell context in which to study BMP signaling. Manipulation of BMP signaling in mESCs is a key step in many directed differentiation protocols,,$\frac{18}{26}$ and single BMPs and pairs can produce distinct cell fates in the same protocol ${ }^{3}$ mESCs also differ from NMuMG cells in their expression of three of the five BMP receptors, providing a different BMP receptor context (Figure 2.4A). Finally, the two cell types are known to respond in distinct ways to some ligand combinations. $\stackrel{4}{4}$

A

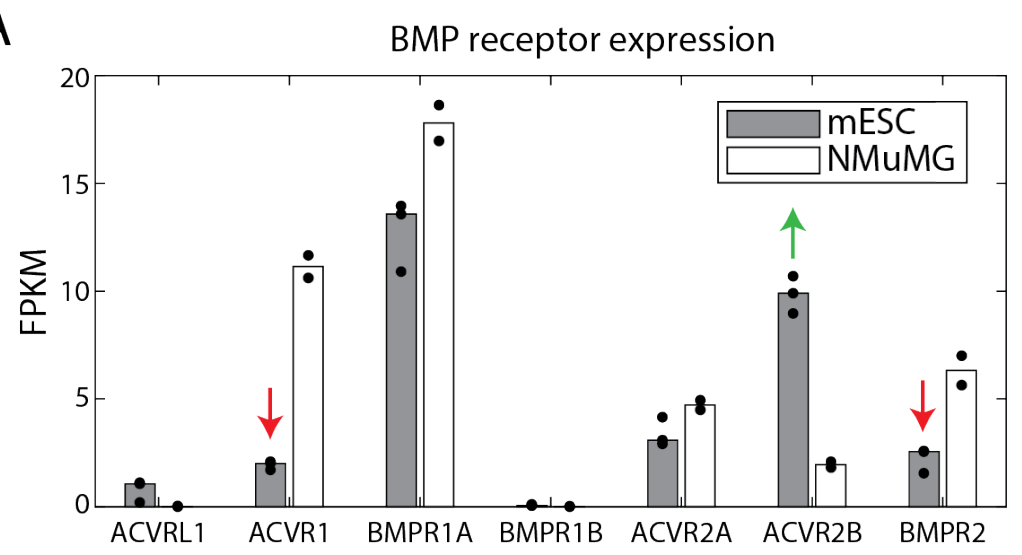

C

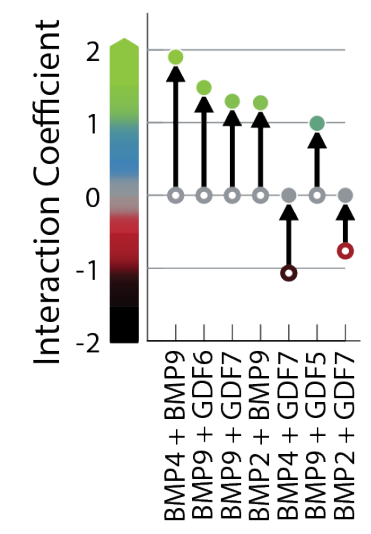

B

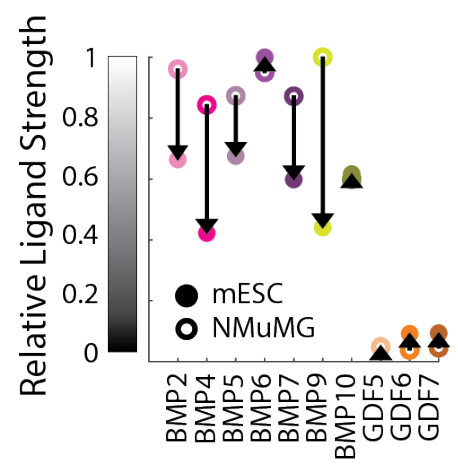

$E$
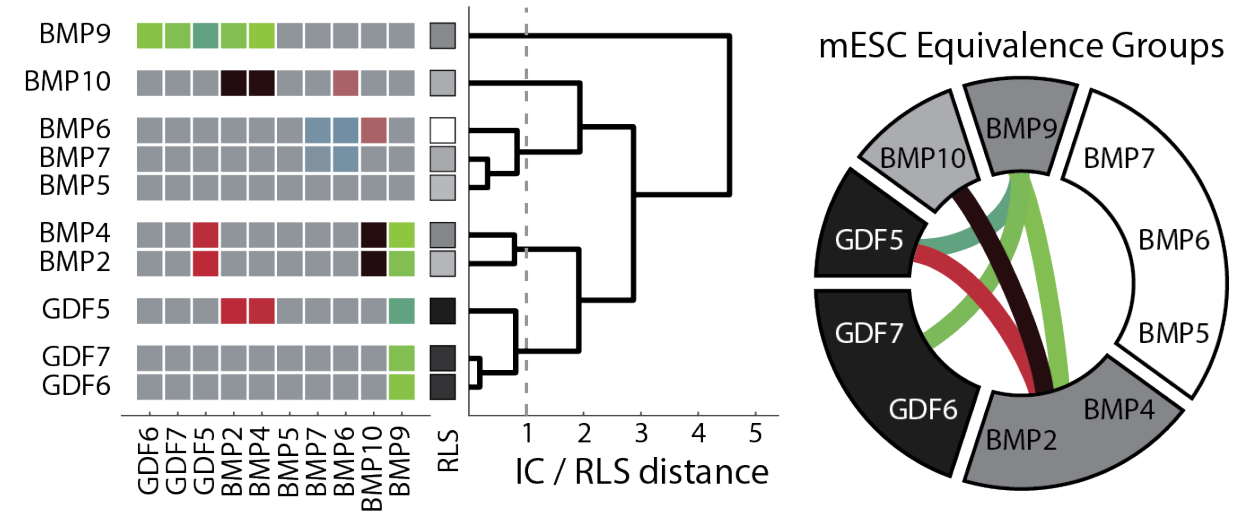

mESC Equivalence Groups

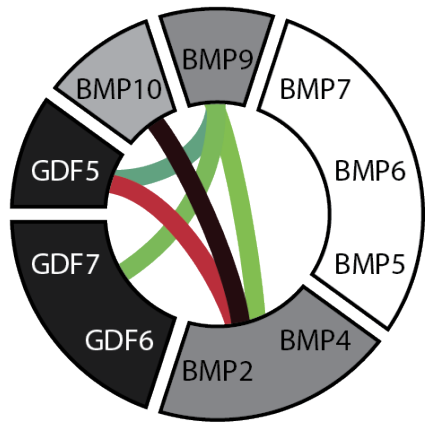

Figure 2.4: Mouse ES cells exhibit distinct BMP equivalence groups. (caption on following page) 
Figure 2.4 (previous page):

(A) RNA-Seq of BMP receptors in mESC reporter and wild-type NMuMG cells show differences in receptor expression (highlighted by green and red arrows) between the two cell lines, with mESCs expressing more ACVR2B, but less ACVR1 and BMPR2. Dots show at least two biological repeats, and bar height is median.

(B) RLS values in mESCs (closed circle) differ from NMuMG cells (open circle, cf. Figure $\mathrm{S} 2.1 \mathrm{G})$, with more intermediate strength activators in mESCs. Arrows indicate the change in mESCs relative to NMuMG cells.

(C) IC values for seven pairwise interactions changed significantly $(|\Delta I C|>0.5)$ between NMuMG cells (open circle) and mESCs (closed circle). The majority show a net increase of IC, producing five new synergistic interactions. Both the color and y-axis value of the open and closed circles indicate the IC value for the indicated cell lines, computed on the median of four biological repeats.

(D) Hierarchical clustering of IC and RLS values in mESCs reveal a new set of ligand equivalence groups compared to NMuMG cells (cf. Figure 2.3D), using the same cutoff criteria to group ligands by similar individual and pairwise interactions (STAR Methods). The square colors indicate either the ligand's IC value when paired with another ligand or the RLS grayscale for its individual strength (see y-axis colormaps in B and C), as indicated by x-tick labels. The dendrogram on the right-hand side shows complete-linkage clustering of Euclidean distance between ligand features.

(E) BMP equivalence in mESCs, as classified by hierarchical clustering in D, includes six equivalence groups. Grayscale value indicates the approximate RLS of the grouped ligands, while connecting line colors indicate the pairwise interactions between groups of ligands. Groups without a linking edge interact additively.

See also Figure $\mathbf{S 2 . 3}$.

We used a fluorescent mESC reporter line to analyze the dose response for each of the ten ligands (STAR Methods). mESCs showed strikingly different responses to the individual ligands compared to NMuMG cells. They exhibited a greater diversity of activation strengths (Figure 2.4B), as well as greater EC50 values and lower Hill coefficients, indicating larger input dynamic ranges with lower sensitivity to moderate ligand concentrations (Figure $\mathrm{S} 2.3 \mathrm{~A}$ ). In particular, high concentrations (approaching $10 \mu \mathrm{g} / \mathrm{mL}$ ) of some ligands failed to fully saturate the response.

Ligand interactions also differed between the two cell lines. While most pairwise interactions remained saturated additive (Figures $\mathrm{S} 2.3 \mathrm{~B}-\mathrm{D}$ ), a subset showed marked differences $(|\Delta I C|>0.5)$ between the two cell lines (Figure 2.4C). Most notably, BMP9 acquired synergistic interactions with activating ligands [BMP2, BMP4] as well as with non-activating ligands [GDF5, GDF6, GDF7] (Figure $\mathrm{S} 2.3 \mathrm{E}$ ), an effect proposed to emerge when ligands in pairs, but not individually, exclusively form high efficiency complexes, due to competition for a limited receptor pool $\stackrel{4}{4}$ At the same time, strong antagonistic interactions between GDF ligands and [BMP2, BMP4] were lost. Together, these shifts represented an overall decrease in antagonism and increase in synergy 
across all ligand pairs (Figure 2.4C). Combined with the changes in individual activation, these differences generated new ligand equivalence relationships (Figure 2.4D). While some groups and interactions were preserved, such as the suppression between [BMP2, BMP4] and [BMP10], many other groups were split or merged (cf. Figures 2.4, $2.3 \mathrm{D}$ ).

Together, these results directly demonstrate the contextuality of ligand interactions and ligand equivalence relationships. Within this larger set of pairwise interactions, a randomly chosen pair of ligands is most likely to interact additively, but is nevertheless unlikely to function equivalently in terms of its individual potency and pairwise interactions with other ligands. Moreover, equivalence groups can change in new cell types. Therefore, an observation of equivalence in one cell context does not guarantee equivalence in another, and vice versa.

\subsubsection{ACVR1 knockdown recapitulates features of mESC ligand interactions}

A key factor that could explain differences in ligand equivalence between NMuMG cells and mESCs is receptor expression. 20152177 $\mathrm{mESCs}$ express less ACVR1 and BMPR2, but more ACVR2B, than NMuMG cells (Figure 2.4A). Out of these three receptors, we first focused on ACVR1, a Type

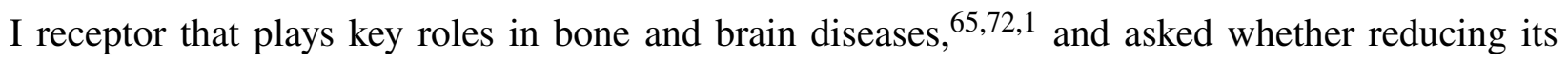
expression could make ligand responses in NMuMG cells more similar to those of mESCs. To achieve stable knockdown of ACVR1, we constitutively expressed shRNA against ACVR1 in the NMuMG reporter cell line. This perturbation reduced expression to less than $20 \%$ of wild-type levels with minimal effects on off-target receptors (Figure S2.4A) and had the same effects on ligand interactions as transient siRNA knockdown of ACVR1 (Figure S2.4B).

ACVR1 knockdown recapitulated some of the observed differences between NMuMG cells and mESCs, particularly those involving BMP9, a ligand known to signal through ACVR1 ${ }^{[72}$ BMP9, the strongest individual activator of NMuMG cells, signaled only weakly in the knockdown cells, consistent with its low activity in mESCs (Figure 2.5A, cf. 4B). More dramatically, ACVR1 knockdown reproduced the synergy of BMP9 with GDF5, GDF6, and GDF7 observed in mESCs (Figures 2.5 B, S2.4 G,H, cf. 2.4 C). Finally, in both mESCs and ACVR1 knockdown, the equivalence of BMP9 with BMP5, BMP6, and BMP7 was eliminated (Figures 2.5 C,D, cf. 2.4E).

By contrast, other distinct features of the mESC response did not appear in the ACVR1 knockdown. Certain non-additive interactions in NMuMG cells persisted even after ACVR1 knockdown (Figures 2.5C, S2.4C-E). For example, [BMP2, BMP4] was still suppressed by [BMP10] (Figures 2.5C, S2.4F) and antagonized by GDF5 (Figure 2.5C). Evidently, significant expression of ACVR1 is not necessary for these non-additive interactions. ACVR1 knockdown also produced new interactions not observed in NMuMG cells or mESCs. [BMP9] antagonized, rather than synergizing with, the other strongly activating ligands, including [BMP2, BMP4] and [BMP5, BMP6, BMP7]. This 

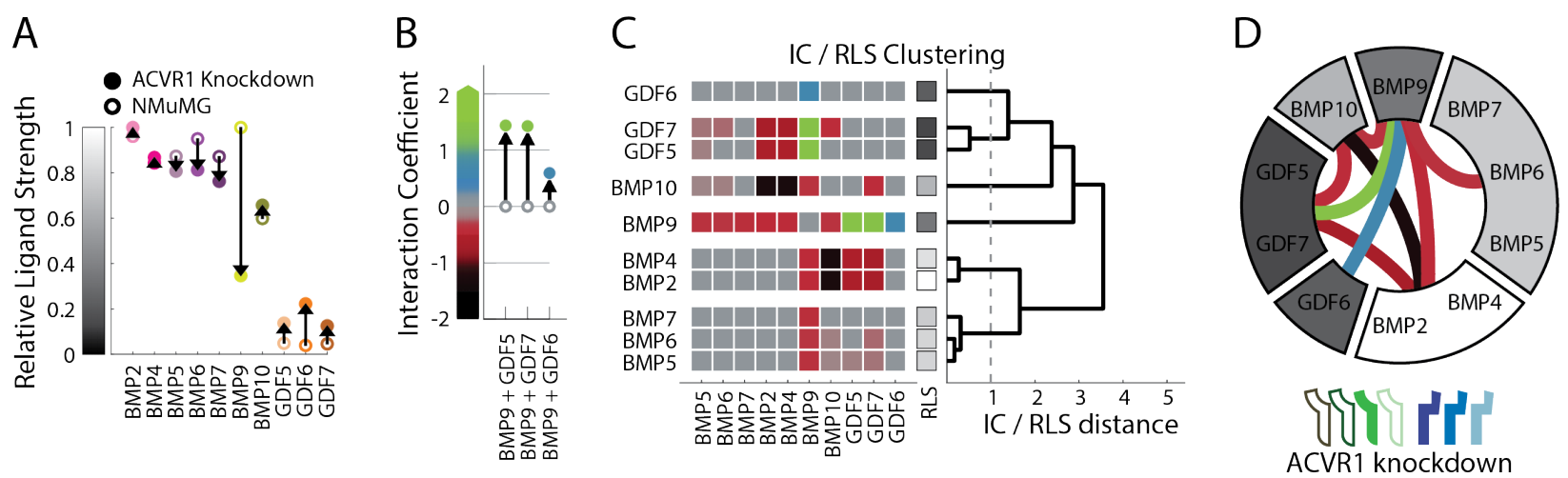

Figure 2.5: ACVR1 knockdown reveals the effects of receptor context on ligand equivalence.

(A) ACVR1 knockdown (closed circle) alters RLS values compared to NMuMG cells (open circle, cf. Figure S2.1G). BMP9 is the most strongly affected.

(B) Three pairwise interactions changed significantly $(|\Delta I C|>0.5)$ between NMuMG cells (open circle) and ACVR1 knockdown (closed circle), producing new synergistic interactions with BMP9. Open and closed circles are colored by IC value.

(C) Hierarchical clustering of RLS and IC values (as in A and B) in the ACVR1 knockdown context shows a more complex equivalence map. The square colors indicate either the ligand's IC value when paired with another ligand or the RLS grayscale for its individual strength (see y-axis colormaps in A and B), as indicated by x-tick labels. The dendrogram on the right-hand side shows complete-linkage clustering of Euclidean distance between ligand features.

(D) BMP equivalence following ACVR1 knockdown, as classified by hierarchical clustering in C, includes six groups. Wedge color shows approximate RLS value of the contained ligands, while connecting line colors indicate pairwise interactions between groups of ligands. Groups without a linking edge interact additively. Here, as in panels E-H, each receptor perturbation is shown as a cartoon of filled and empty receptors, which are drawn in the following order: ACVRL1, ACVR1, BMPR1A, BMPR1B, ACV2A, ACVR2B, BMPR2.

See also Figure $\mathbf{S} 2.4$

result therefore also showed that a single ligand (BMP9) can produce interactions of opposite signs in the same cell context, which was also not observed in either NMuMG cells or mESCs. Together, these results suggest that differences in receptor expression, and ACVR1 expression more specifically, can account for some of the distinct ligand responses of mESCs and NMuMG cells, and reveal that knockdown of a single receptor subunit can substantially increase the complexity of ligand interactions.

\subsubsection{Perturbations of multiple BMP receptors reveal flexibility of ligand equivalence}

To further explore the effects of single receptor perturbations on ligand equivalence, we individually knocked down the two most abundant receptors, BMPR1A and BMPR2, or ectopically expressed the two receptors with weakest endogenous expression, ACVRL1 and BMPR1B. These four re- 
ceptors differ widely in their ligand selectivity and expression profiles. BMPR2 and BMPR1A bind a diverse set of BMPS $50|45| 49$ while ACVRL1 and BMPR1B have more specific ligand preferences. Additionally, BMPR1A and BMPR2 are broadly expressed across distinct tissues, whereas BMPR1B and ACVRL1 are highly expressed in relatively few tissues. ${ }^{20 \mid 19}$ We reasoned that perturbing receptors with such diverse properties could provide insight into how different specificities of receptor-ligand interactions influence ligand equivalence.

To explore these diverse receptor properties, we first generated stable shRNA knockdown cell lines for BMPR1A and BMPR2, similar to the ACVR1 line (Figures S2.5A,B). Knockdown of BMPR2, which is also reduced in mESCs relative to NMuMG cells, decreased activation by BMP2 and BMP4, removed their antagonism by GDF5 and GDF7, and fused [GDF5, GDF6, GDF7] into one equivalence group (Figures 2.6A "BMPR2 KD," S2.5C-G), all of which were features of BMP signaling in mESCs. Thus, this perturbation recapitulated certains aspects of mESC equivalence, though distinct from those produced by ACVR1 knockdown. Knockdown of BMPR1A produced a further simplified version of the BMPR2 knockdown, with no activation by BMP2 and BMP4 and only one non-additive interaction occurring between two of the three groups (Figures 2.6A "BMPR1A KD," $\mathrm{S} 2.5 \mathrm{H}-\mathrm{J})$. The loss of most non-additive interactions following knockdown of BMPR2 or BMPR1A also revealed that these receptors, unlike ACVR1, are necessary for many non-additive ligand interactions in NMuMG cells.

Thus far, perturbations were of relatively promiscuous receptors, known to interact with a broad spectrum of ligands. By contrast, the receptors ACVRL1 and BMPR1B are known to be more ligand-specific, ${ }^{14 / 45 \mid 19}$ primarily binding BMP9 and BMP10 or the GDFs, respectively. We therefore ectopically expressed these receptors by stably integrating constructs encoding receptor cDNA into the NMuMG reporter cell line (Figure S2.6A). Ectopic ACVRL1 expression increased activation by BMP10 and removed its many suppressive and antagonistic interactions with other ligands (Figures 2.6A “ACVRL1 OX,"S2.6B-D). Similarly, BMPR1B increased activation by its preferred ligands GDF5, GDF6, GDF7, and removed their antagonism of BMP2 and BMP4 (Figures 2.6A "BMPR1B OX," S2.6E-H). Thus, both perturbations reduced the number of equivalence groups and non-additive interactions, with ectopic BMPR1B expression allowing 8 of the 10 ligands to signal equivalently. More specifically, ectopic expression of each receptor resulted in its preferred ligands becoming equivalent to other strongly activating, weakly interacting ligands, such as BMP5, BMP6, BMP7, and BMP9. These experiments suggest that non-additive interactions could arise from receptor competition, which is specified by the receptor's ligand preferences and can be relieved by ectopic receptor expression. 


\subsubsection{Comparing signaling across cell types reveals global structure of ligand equivalence}

Together, the pairwise interactions and corresponding equivalence maps observed across all cell contexts provide a more global view of the structure of BMP signaling. The full set of pairwise interactions reveals the striking plasticity of ligand strength and interactions (Figure 2.6A, top). GDF5, GDF6, and GDF7 were usually weak activators, while BMP5, BMP6, and BMP7 strongly activated all cell types. The remaining ligands were active in some but not all receptor contexts. While most pairwise interactions were additive, every ligand participated in synergy or antagonism with another ligand in at least one receptor context. Non-additive interactions tended to be consistent for a given ligand pair, exhibiting the same interaction type (i.e. color) across cell lines. All synergistic ligand interactions involved BMP9, whereas all suppressive interactions involved BMP10. Considered as a system, the meaning of any given BMP ligand is thus inherently contextual, depending both on other ligands and on receptor context.

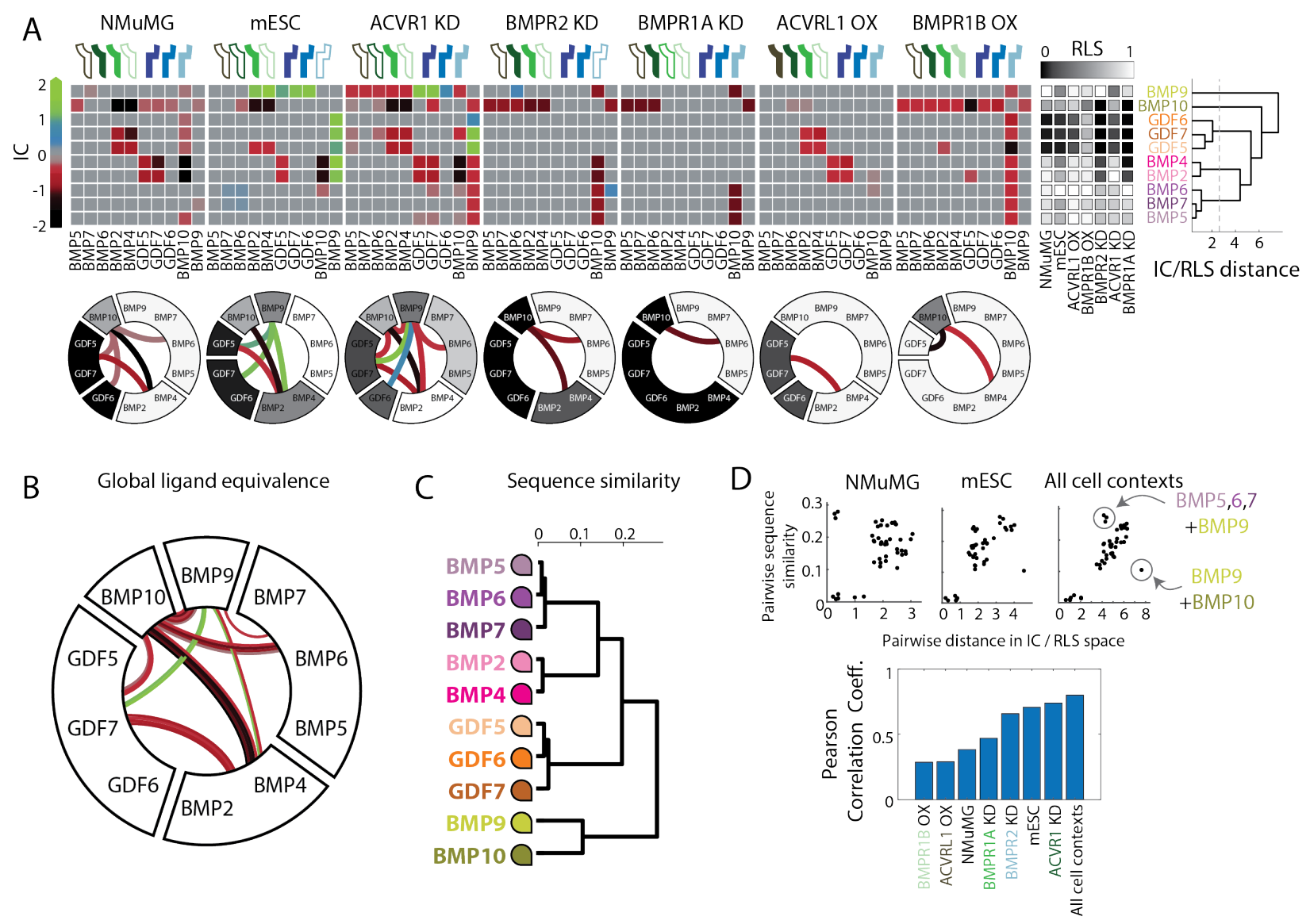

Figure 2.6: Analysis of multiple cell contexts reveals global ligand equivalence groups. (caption on following page) 
Figure 2.6 (previous page):

(A) Clustering RLS and IC values for all ligands and pairs across all cell contexts shows global ligand similarity (upper row). The full set of IC values show the distribution of ligand interaction types (colors based on Figure 2.3C) and identify ligands with different frequencies of non-additive interactions. Lower row shows the corresponding ligand equivalence groups (reproduced from Figures 2.3 $\mathrm{D}, 2.4 \mathrm{E}, 2.5 \mathrm{D}-\mathrm{H}$ ). For the dendrogram (right), clustering was done by complete linkage of the Euclidean distance between RLS and BC features, as before.

(B) The global equivalence map was determined by cutting the dendrogram (panel A, right) at the indicated distance. Internal links show all instances of non-additive interactions between those ligands. Global groups tend to have linkages of the same interaction type (i.e. similar colors), with widely varying frequency. For example, BMP2 and BMP4 almost always interact with BMP10 but never with BMP5.

(C) Recombinant ligand sequences were clustered by complete-linkage clustering of pairwise alignment distance, as computed by the BLOSUM50 matrix between globally-aligned sequences, neglecting all gaps. Ligands comprise roughly four groups of related proteins, indicated by color families. The recombinant ligands used in this study constitute the mature secreted form of the ligand, exclude pro-domains, and vary in length from 108 to 146 amino acids (see also Figure S2.7A).

(D) Sequence similarity, quantified as in (C), correlates with IC/RLS similarity observed in each cell context, though the extent of correlation varies. In the correlation across all cell contexts, notable outliers include BMP9's surprising functional difference from BMP10, despite their significant sequence similarity, and BMP9's unexpected similarity with BMP5, BMP6, BMP7, despite relatively dissimilar sequences. Pearson correlation coefficient (bottom) quantifies correlation between sequence similarity and IC/RLS distance for each cell line. Highest correlation is achieved by considering all cell contexts together.

See also Figures S2.5, S2.6, $\mathrm{S} 2.7$

To represent the full dataset in a more compact way, we clustered ligands according to the similarity of their interactions across receptor contexts (Figure 2.6A, right), such that ligands in the same cluster had the same individual strength and pairwise interactions across all receptor contexts. This approach classified the ten BMPs into five global equivalence groups: [BMP2, BMP4], [BMP5, BMP6, BMP7], [BMP9], [BMP10], and [GDF5, GDF6, GDF7]. [BMP2, BMP4], as well as [BMP5, BMP6, BMP7], exhibited the strongest version of equivalence. Despite frequent changes in individual or combinatorial signaling across cell contexts, responses to ligands within each group always changed in the same manner, such that the ligands in each group were equivalent in all contexts. The global equivalence map also illustrates the variability and prevalence of ligand interactions (Figure 2.6B). For example, interactions between [BMP9] and [BMP2, BMP4] can vary from synergy to antagonism. In all receptor contexts except one, [BMP10] interacts non-additively with [BMP2, BMP4], while [BMP5, BMP6, BMP7] have only additive interactions with [BMP2, 
BMP4] or [GDF5, GDF6, GDF7]. (Note that we grouped GDF6 with GDF5 and GDF7 despite its apparent lack of antagonistic interaction with [BMP2, BMP4] because its higher overall EC50 may obscure a potential underlying antagonism (Figure S2.6E, inset; STAR Methods).)

This global equivalence diagram also portrays another surprising feature of context-dependent ligand differences. A single linear ligand ordering, following the circle from BMP9 around to BMP10, is consistent with all observed equivalence maps. With this ordering, no rearrangements of ligand position are necessary in any observed context. This suggests that ligand differences fall on a linear continuum, and is distinct from what one would expect in a clustering-based analysis, where many orderings are consistent with the same linkage tree. While analysis of additional receptor contexts could further increase the number of global equivalence groups, this diagram efficiently summarizes ligand differences and interactions.

How closely do the equivalence relationships assessed here reflect intrinsic aspects of ligand sequence? To assess this, we compared similarity of ligand protein sequence (Figures 2.6C, S2.7A) with similarity of pairwise interaction profiles. In individual cell lines, these two metrics were only partially correlated (Figure 2.6D, top), suggesting that ligand sequence similarity does not fully predict functional similarity in a single receptor context. For example, BMP5 and BMP9 act equivalently in NMuMG (Figure 2.3D) but are relatively dissimilar in sequence (Figure 2.6C). Ligand sequence correlated with ligand function more strongly when all cell lines were considered simultaneously (Figure 2.6D, bottom). This 'global' correlation coefficient, 0.80, approached the maximum value obtained with randomly sampled cell line combinations, 0.84 (Figure S2.7B). Interestingly, however, even when all cell lines were considered together, BMP9 and BMP10 exhibited more divergent functional behavior than expected given their sequence similarity. Overall, this analysis suggests that ligand sequence similarity does not generally reflect functional similarity in any individual cell context, even for the most highly homologous ligands. Instead, it appears to correlate more strongly with aggregated, or global, functional similarity across multiple contexts.

\subsubsection{A mathematical model of competitive ligand-receptor interactions can explain context- dependent ligand interactions}

As shown above, ligands can interact in different ways depending on receptor context. To understand how such complex, contextual responses could emerge, we analyzed a mathematical model of the

pathway developed in a parallel study. ${ }^{68}$ This model assumes that each ligand variant, $L_{i}$, can bind to any pair of Type I and Type II receptors, $A_{j}$ and $B_{k}$ respectively, with affinity $K_{i j k}$ to produce a trimeric, ligand-receptor signaling complex, $T_{i j k}$ (Figure 2.7A), whose binding is captured by a set of ordinary differential equations describing mass-action kinetics. Each signaling complex can then phosphorylate SMAD proteins with its own specific kinase activity $\epsilon_{i j k}$. Receptors are 
expressed at specific total levels, $A_{j}^{0}$ or $B_{k}^{0}$. The limited total amount of each receptor generates competition among ligands for available receptors. Other features of the BMP system-including step-wise assembly of ligand-receptor complexes, the heterotetrameric stoichiometry of actual receptor complexes, co-receptors, and other factors-play important roles in the natural system but were not required to explain observations in this work (STAR Methods). 

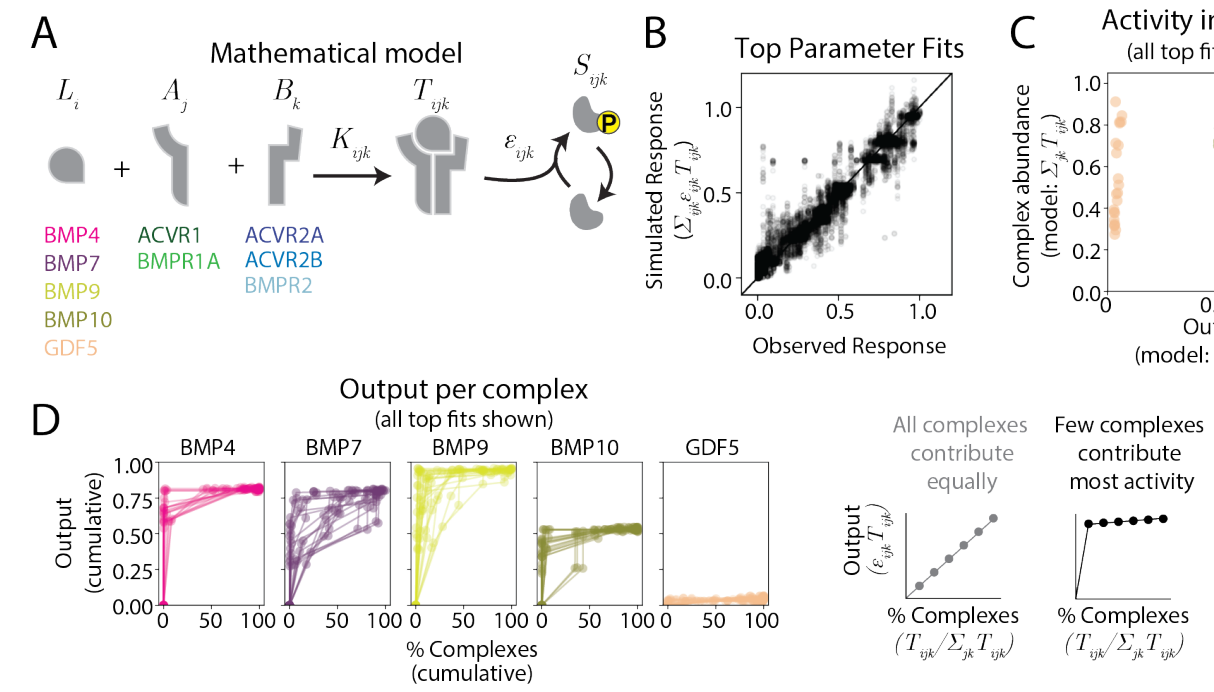

E Single receptor knockdown can indirectly reduce output from every complex

$\mathrm{F}$

C. Activity in NMuMG
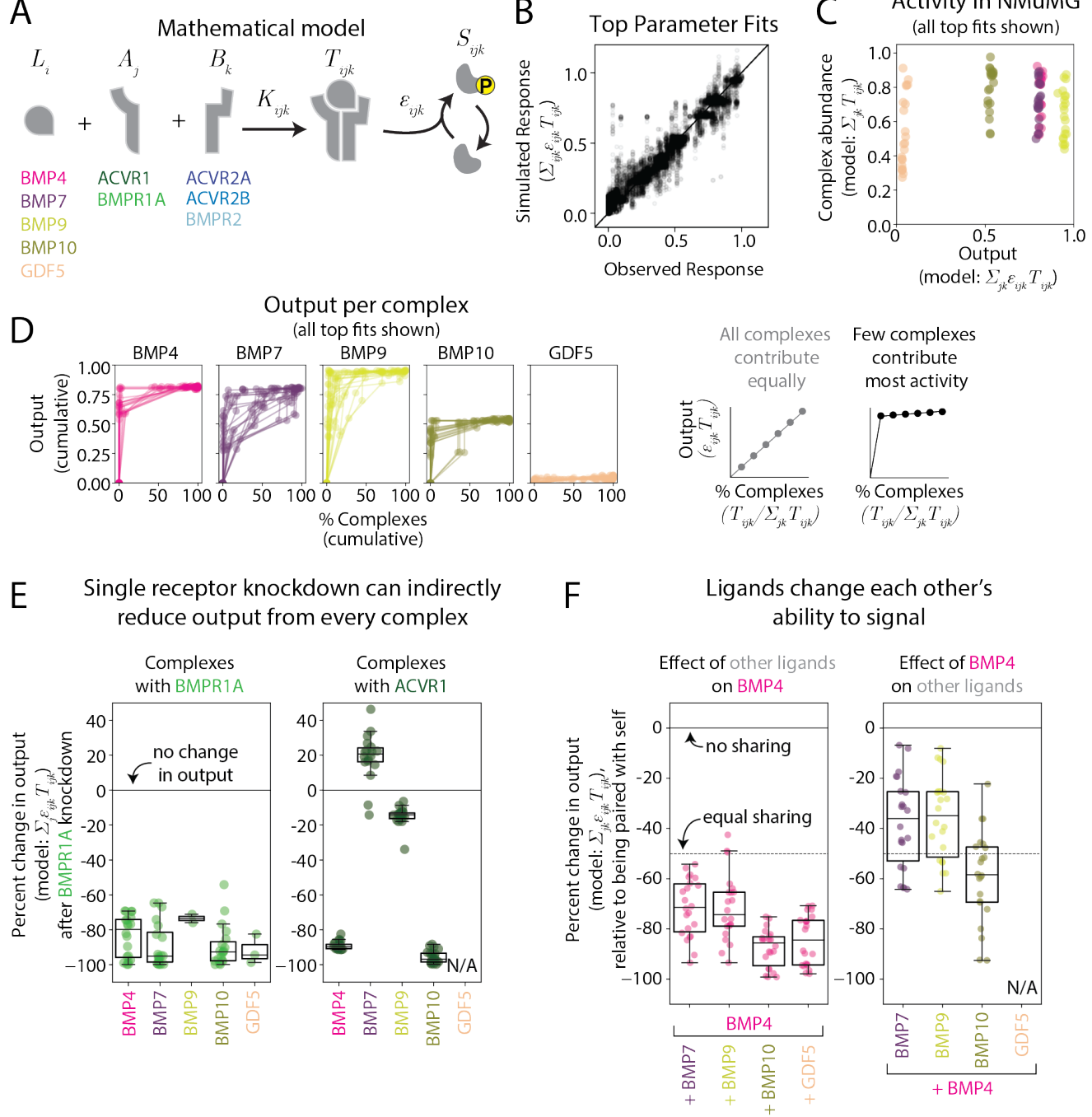

Ligands change each other's ability to signal
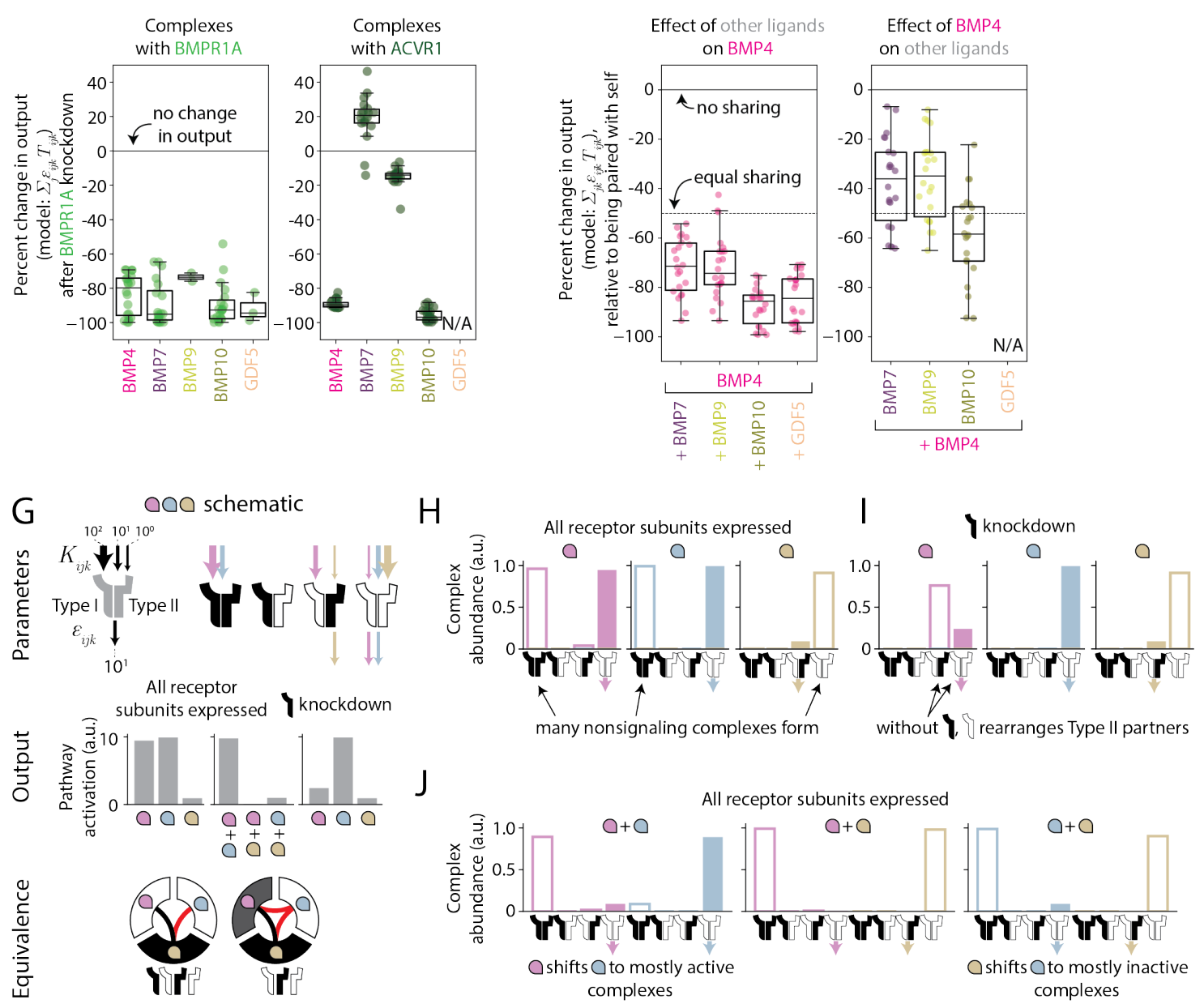

Figure 2.7: Mathematical model of receptor competition can explain contextual ligand equivalence groups. (caption on following page) 
Figure 2.7 (previous page):

(A) In a minimal BMP receptor competition model, mass action kinetics govern one-step assembly of ligand $\left(L_{i}\right)$, Type I receptor $\left(A_{j}\right)$, and Type II receptor $\left(B_{k}\right)$ into trimeric signaling complexes $\left(T_{i j k}\right)$ that activate pathway output with some activity $\left(\epsilon_{i j k}\right)$. The included components are listed beneath their associated variable.

(B) Observed responses, normalized between 0 and 1, correlate with simulated responses in top parameter fits.

(C) Total complex abundance (in arbitrary units, normalized to total possible number of complexes) does not correlate with overall output from a given ligand. Ligands of intermediate strength (e.g. BMP10) can bind more receptors than strongly activating ligands.

(D) For individual ligands activating NMuMG, the inferred output of each complex is plotted against its percentage of the total complexes for all top parameter fits. A minority of the complexes that form can produce the majority of the output.

(E) The percent change in output (relative to NMuMG) from BMPR1A- and ACVR1-containing complexes following BMPR1A knockdown is shown for all five ligands. BMPR1A knockdown reduces the number of BMPR1A-containing complexes and reduces their output as expected, but also can reduce output from ACVR1-containing complexes, as BMPR1A loss increases availability of Type II receptors. All top parameter fits are plotted, unless the absolute change is small (i.e. $<0.05$ change in response). GDF5, which does not activate these cells and has only small changes in ACVR1 output, is not shown (N/A).

(F) Percent change in output (relative to signaling alone) from complexes containing BMP4 or another ligand are shown for BMP4 paired with four other ligands: BMP7, BMP9, BMP10, and GDF5. Equal sharing between the two ligands would reduce output by 50\%, whereas ligands that do not share complexes produce as much as output alone as they do in a pair. All top parameter fits are plotted, unless the absolute change is small (i.e. <0.05 change in response). GDF5, which does not activate these cells and has only small changes in overall output, is not shown (N/A).

(G) A schematic parameter set shows three ligands with different affinities and activities for four possible receptor dimers. In the model, these ligands have different individual strengths and pairwise strengths which change with receptor perturbations.

(H) In a simulation where each receptor subunit is available, the three ligands form both signaling and nonsignaling complexes. Bar height shows complex abundance. Empty bars show nonsignaling complexes, whereas filled bars show signaling complexes.

(I) After simulated knockout of the black Type I receptor, the abundances of many signaling complexes change, even if they did not involve the knocked out receptor.

(J) In a simulation where each receptor subunit is available, competition for shared receptors changes the formation of signaling complexes by each ligand. The pink and gold ligands can respectively shift the blue ligand to preferentially form its signaling and nonsignaling complexes.

See also Figure $\mathbf{S} 2.8$.

To identify parameter values that can generate the range of interactions observed experimentally, we focused our analysis on a single ligand from each global equivalence group. Similarly, we considered only the five broadly-expressed receptors (Figures 2.7A, S2.4A, S2.5A): the Type I 
receptors ACVR1 and BMPR1A and the Type II receptors ACVR2A, ACVR2B, and BMPR2. We fit the model simultaneously to measurements of individual and pairwise responses in the four NMuMG-derived cell lines whose receptor profiles are restricted to this set (i.e. NMuMG and the knockdowns of ACVR1, BMPR2, and BMPR1A). We then analyzed a set of 22 solutions from nearly 7,000 least-squares fits that produced the lowest fitting error and preserved key pairwise interactions (Figures 2.7B, S2.8A; STAR Methods).

Within these best fit parameter sets, all ligands were promiscuous, with substantial but variable affinities for most or all receptors (Figure S2.8B, top). BMP7 and GDF5 had lower affinities overall across all complexes, while BMP4, BMP9, and BMP10 had higher affinities for select complexes (Figure $\mathrm{S} 2.8 \mathrm{~B}$, top). The ligands also differed in the activity of the resulting complexes. GDF5 weakly activated most signaling complexes, while other ligands strongly activated multiple receptor complexes (Figure $\mathrm{S} 2.8 \mathrm{~B}$, bottom). Many signaling complexes exhibited strong affinity but weak activity or vice versa (Figure S2.8C). A parallel computational study of the BMP pathway showed that this inverse relationship between activity and affinity generates the complex multi-ligand responses necessary for combinatorial addressing, in which ligand combinations can selectively activate cell types. ${ }^{68}$ Taken together, these results suggest that the context-dependent ligand interactions observed experimentally can be explained by affinity-based competition to form complexes with different activities. They further suggest that the effective biochemical parameters for mammalian BMPs exhibit the features associated with combinatorial addressing.

\subsubsection{In the model, context-dependence emerges from redistribution of signaling complexes}

We identified three key features of the best fit parameter sets that allowed them to reproduce the observed context-dependent ligand interactions. First, each of these parameter sets generated many high affinity (high $K_{i j k}$ ), low activity (low $\epsilon_{i j k}$ ) signaling complexes. Ligands can appear weak not because they form fewer complexes but rather because they preferentially form complexes with weaker activity (Figure $2.7 \mathrm{C}$ ). More surprisingly, even for highly active ligands, most of the response was generated by a small fraction of the formed complexes (Figures $2.7 \mathrm{D}, \mathrm{S} 2.8 \mathrm{E}, \mathrm{F}$ ).

A second feature of top parameter fits was that they allowed individual receptor subunits to participate in multiple complexes with widely varying activities. Consequently, when receptors are rearranged into new complexes following the perturbation of a single receptor, significant changes in pathway activity can result. These changes can be direct and indirect. For example, knockdown of a single receptor subunit can directly reduce the output from complexes in which it appears by reducing their concentrations. It can also indirectly affect the response from other signaling complexes by increasing or decreasing abundance of inactive, competing complexes (Figures 2.7E, $\mathrm{S} 2.8 \mathrm{G}, \mathrm{H})$. For example, in the model, knockdown of BMPR1A reduces the activity of BMP4 and 
BMP10 complexes containing ACVR1 (and lacking BMPR1A itself). This indirect effect is due to the release of ACVR2B from BMPR1A, which allows formation of ACVR1-ACVR2B complexes that compete with the higher-activity ACVR1 complexes (Figures $\mathrm{S} 2.8 \mathrm{G}, \mathrm{H}$ ). These indirect effects account for much of the complexity of receptor context.

Finally, the parameter fits also produced hidden, or compensatory, shifts in the activity of one ligand in response to addition of a second ligand. For example, in NMuMG cells, BMP4 combined additively with BMP7 (Figure 2.3D), seemingly implying that the two ligands do not affect each other's ability to signal. However, in nearly all top fitting results, BMP7 produced more output than expected if it shared its receptors equally with BMP4, while BMP4 produced less than expected. Thus, the saturated additive BMP4-BMP7 interaction resulted from opposite effects on the underlying signaling activities of the two ligands (Figures 2.7F, S2.8[,J). A more complete analysis of redistribution of signaling complexes is shown in Figures $\mathrm{S} 2.8 \mathrm{D}-\mathrm{J}$, which highlight a single random parameter set.Taken together, these results show how reassortment of ligand and receptor subunits can reproduce observed interactions through sometimes non-intuitive mechanisms.

To better illustrate how these effects occur, we constructed a simplified hypothetical system with three ligands, two Type I, and Type II receptor subunit variants interacting with the affinity and activity parameters shown in Figure $2.7 \mathrm{G}$. As observed in the parameter fits, when all receptor subunits are expressed, even the highly active pink and blue ligands form many non-signaling complexes (Figure 2.7 H). Further, knocking down a receptor indirectly reduces output from unperturbed receptors (Figure 2.7I). For example, when the black Type I receptor is removed, the white Type I receptor obtains unrestricted access to the pool of Type II receptors, allowing formation of a new, lower-activity complex with the pink ligand. Thus, even though the white Type I receptor is not itself perturbed, its output is reduced.

This schematic example also shows how addition of one ligand can modify the activity of another through competition. For example, the gold ligand competes with the blue ligand to bind to the white Type I and II receptor, which is the only receptor through which the blue ligand can signal (Figure 2.7J), producing an antagonistic interaction between the two ligands. By contrast, when the blue and pink ligands are combined, signaling by the former is increased while signaling by the latter is decreased relative to their individual activities. These changes cancel each other out, resulting in minimal change to the total signal. In this case, the apparent additivity of the interaction conceals compensatory changes in the distributions of both ligands. Thus, competition generally redistributes ligands across receptor complexes but does not necessarily produce changes in total signaling activity. This simplified example illustrates many of the principles observed in the full model. 


\subsection{Discussion}

Individual BMP ligands are known to preferentially bind and signal through specific receptors. However, preferences alone cannot explain the striking contextuality of BMP ligand responses. Here, we introduced contextual ligand equivalence groups as an alternative framework to understand ligand differences when signaling in combinations across receptor contexts. This approach uses systematic pairwise measurements to define equivalence groups, each comprising a set of ligands that interact similarly with all other ligands when activating a given cell type (Figure 2.3D). Critically, these equivalence groups are contextual, changing with alterations in receptor expression (Figures 2.4[2.5[2.6]. In contrast to ligand classifications based on biochemical properties like affinity, contextual equivalence groups classify BMP ligands and receptors based on their emergent functional properties.

Contextual equivalence groups can explain ligand redundancy that varies between developmental contexts. For example, BMP10 signals in both developing heart and vasculature. ${ }^{15}$ Although BMP9 has the closest sequence similarity to BMP10, the contextual equivalence maps show that BMP9 can only substitute for BMP10 in the presence of ACVRL1 (Figure 2.5 G). Consistent with this picture, BMP9 rescued loss of BMP10 in developing mouse vasculature but not heart, as ACVRL1 expression is limited to vascular epithelium. ${ }^{15 / 63}$ Contextual equivalence groups also reveal the effects of ligand context. For example, in receptor contexts where BMP4 and BMP7 are strong activators, they can only replace each other when antagonizing ligands such as GDF5, GDF6, GDF7, or BMP10 are absent (Figures 2.3D, 2.5 $\mathrm{G}, 2.5 \mathrm{H}$ ). Consistent with this, BMP4 and BMP7 functioned redundantly in the developing kidney, ${ }^{50}$ where those antagonizing ligands are not expressed. ${ }^{25}$ By contrast, loss of BMP4 produced more pronounced effects than loss of BMP7 in the context of developing skeleton with conditional BMP2 knockout, ${ }^{7}$ a context where GDF5 is expressed and plays a key role. ${ }^{67}$

The contextual equivalence analysis of BMP signaling introduced here can be improved and extended in several ways. First, analyzing ligand equivalence in additional receptor contexts would provide more complete coverage of possible BMP signaling contexts. Second, including heterodimeric ligands such as BMP2/7 could reveal whether these ligands have distinct contextual properties from their related homodimers, in addition to their unique individual properties. ${ }^{73}$ Third, many natural contexts use three or more ligands simultaneously. The approach taken here could be extended to combinations of three or more ligands to determine whether pairwise interactions completely determine multi-ligand effects or whether higher-order interactions are important.

Contextual equivalence groups can be explained by a simple biochemical model, suggesting that the context-dependent behavior of BMP ligands could arise from differences in receptor affinity and signaling complex activity. Key aspects of this model are consistent with previously observed 
features of BMP signaling. For example, loss of BMP or related TGF- $\beta$ receptors indirectly

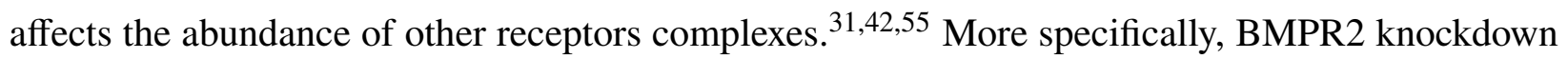
has unexpected and ligand-specific effects across a variety of cell types,, $29|31| 80|47| 71 \mid$ which may be explained by the low predicted activity of BMPR1A-BMPR2 complexes that recurred in all model fits. In pulmonary artery cells and myeloma cells, BMPR2 knockdown either did not affect or decreased signaling by BMP4 and BMP10, while, respectively, increasing or having no effect on signaling by BMP7 and BMP9. In the model, without BMPR2, BMP4 and BMP10 preferentially formed lower activity complexes than with BMPR2. Therefore, the absence of BMPR2 redistributes receptors to reduce pathway activity overall. By contrast, BMP7 and BMP9 mostly form complexes with high activity in the absence of BMPR2. For these ligands, association with other Type II receptors would tend to increase or maintain pathway activation. Thus, while the minimal phenomenological model established here does not represent the full complexity of the BMP system, it provides a foundation for a unified, systems-level framework for describing ligand differences, generating hypotheses, and reconciling otherwise counter-intuitive effects of perturbations among cell types.

Independent experimental measurements of the effective affinity and activity parameters for various ligand-receptor signaling complexes (Figure 2.7) could determine parameters not fully constrained by existing data. This would allow the model to predict responses to ligand combinations in arbitrary cell (i.e. receptor) contexts. More specifically, measuring the effective affinity of overall complex formation may be a crucial follow-up to measuring the affinity of BMP ligands binding single receptors, $\stackrel{30[58|5| 34}{3}$ and measurements of signaling complex activity may provide more examples of BMP ligands that bind and only weakly activate receptors, or vice versa. $\frac{45 / 44 \mid 6}{6}$ Finally, while the existing model shows that competitive ligand-receptor interactions are sufficient to explain key features of contextual equivalence groups, further tests will be essential to understand how additional biochemical interactions, such as binding to negative regulators like Noggin, could enable other pathway behaviors.

Ligand classification by pairwise interactions can generalize beyond the core BMP pathway. First, additional TGF- $\beta$ family ligands, such as TGF- $\beta$ or activin, could be included. These ligands regulate phosphorylation of the related SMAD2/3 transcription factors, share some but not all receptors with BMP ligands, 55 and exhibit distinct activation dynamics that may allow more complex ligand integration. $\frac{76 / 81}{}$ Expanding into this related set of ligands not only introduces the probability of more complex equivalence relationships but also provides deeper insight into a critical signaling system in cancer and wound healing. ${ }^{851}$ Second, analyzing a broader set of BMP targets, including non-canonical outputs and broad repertoires of target genes, could determine whether different outputs follow the same ligand equivalence relationships as SMAD1/5/8 
phosphorylation. 592810 Third, a similar approach could be applied to other pathways, such as Wnt or FGF, which also exhibit promiscuous interactions among multiple ligand and receptor variants. 24/37 In the longer term, contextual equivalence groups could function as a 'periodic table' that organizes ligands according to their functional effects and contextual interactions in a unified manner to explain why specific components operate together in different developmental contexts and to allow context-specific control of pathway activity for therapeutic applications.

\subsection{Acknowledgments}

H.K., Y.E.A., and M.B.E. conceived and designed the experiments. H.K., M.A.L., and J.M.L. performed the experiments. H.K. and M.A.L. analyzed the experimental data. H.K., Y.E.A., and C.J.S. developed and fit the model. H.K., Y.E.A., and M.B.E wrote the paper. This work was supported by the Defense Advanced Research Projects Agency (contract HR0011- 16-0138), the Gordon and Betty Moore Foundation (grant GBMF2809 to the Caltech Programmable Molecular Technology Initiative), the Human Frontiers Science Program (grant RGP0020), the Institute for Collaborative Biotechnologies (grant W911NF-09-0001 from the U.S. Army Research Office), the National Institutes of Health (NIH) (grants R01 HD075335A and R01 MH116508), and the Paul G. Allen Frontiers Group and Prime Awarding Agency (award UWSC10142). This work does not necessarily reflect the position or policy of the U.S. Government, and no official endorsement should be inferred. H.K. is supported by a National Science Foundation graduate research fellowship (grant DGE-1144469). C.J.S. is supported by the NIH National Institute of General Medical Sciences (grant T32 GM008042) and a David Geffen Medical Scholarship. M.A.L. is supported by the National Sciences and Engineering Research Council of Canada Postgraduate Doctoral Scholarship. M.B.E. is a Howard Hughes Medical Institute Investigator. 


\subsection{Supplementary Figures}

A

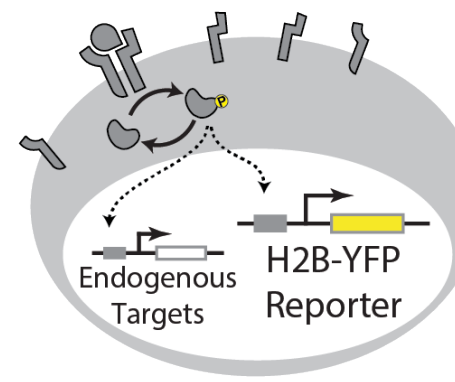

NMuMG Reporter
B

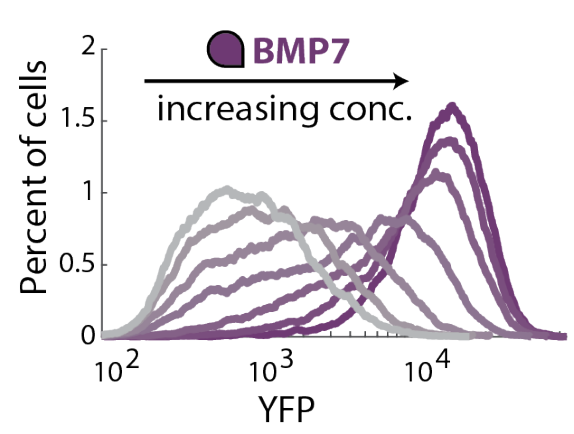

C

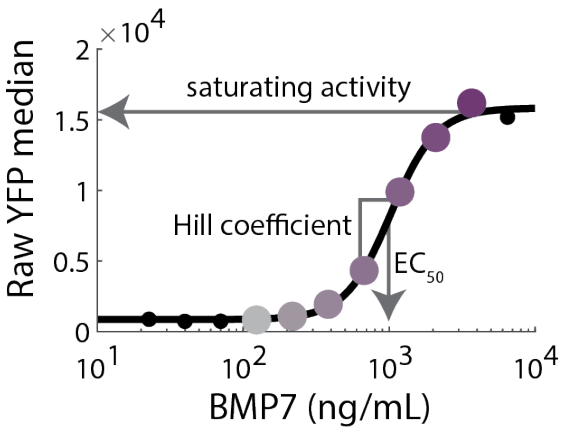

E
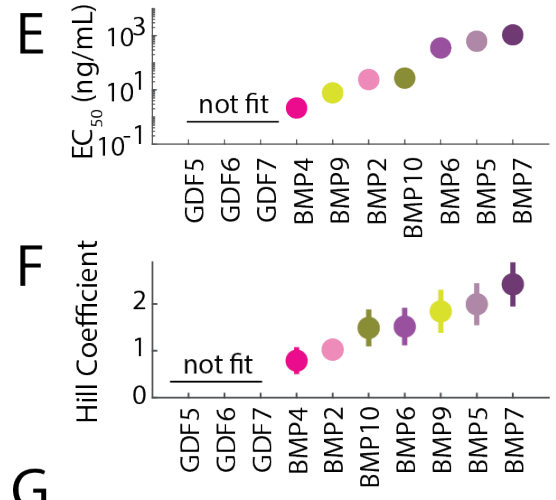

G

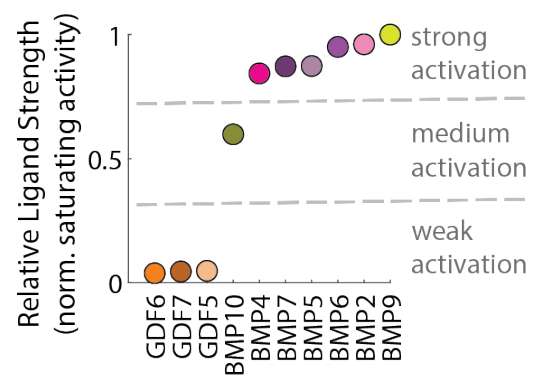

Figure S2.1: Quantitative dose-responses reveal individual ligand differences. (caption on following page) 
Figure S2.1 (previous page):

(A) NMuMG reporter cells include a stably-integrated BMP response element driving expression of Histone 2B-mCitrine (H2B-YFP).

(B) Flow cytometry data (YFP channel) for a BMP7 dose response in the NMuMG reporter cell line shows concentration-dependent increase in YFP. Colors shaded from gray to purple indicate increasing concentrations of BMP7, plotted in C.

(C) Medians of YFP distributions in B show BMP7 dose response, plotted as a function of BMP7 concentrations, which are colored by the same scheme as in B. Black line shows Hill fit of this single biological replicate. Black dots are responses at concentrations not shown in panel B, but acquired in the same manner.

(D) Dose responses for ten BMP ligands in the reporter line vary in slope, EC50, and saturating activity. Dots represent three biological repeats, and lines indicate the best fits to Hill functions.

(E) $\mathrm{EC}_{50}$ values from best fit Hill functions for all activating ligands vary continuously over two orders of magnitude. Parameters for GDF5, GDF6, GDF7, whose weak responses do not resemble Hill fits, were omitted.

(F) Hill coefficients for activating ligands vary over a small range.

(G) Relative Ligand Strength (RLS, saturating ligand activity normalized to the strongest ligand's saturating activity) values show into weak, medium, and strong activation levels.

See also Figure 2.3 . 
A

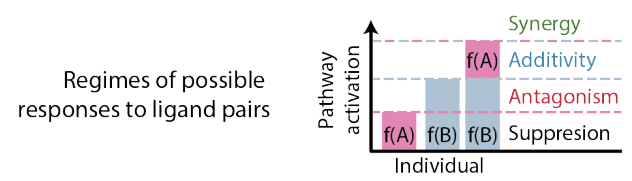

Pairwise interaction regime

Synergy
$f(A)+f(B)<f(A+B)$

Normalized interaction strength IC Range

- $\mathrm{K}=\frac{\mathrm{f}(\mathrm{A}+\mathrm{B})}{\mathrm{f}(\mathrm{A})+\mathrm{f}(\mathrm{B})} \quad 1<\mathrm{KC}$

Additivity

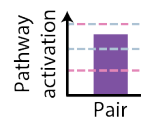

- $\mathrm{K}=\frac{\mathrm{f}(\mathrm{A}+\mathrm{B})}{\mathrm{f}(\mathrm{B})} \quad 0 \leq \mathrm{K} \leq 1$

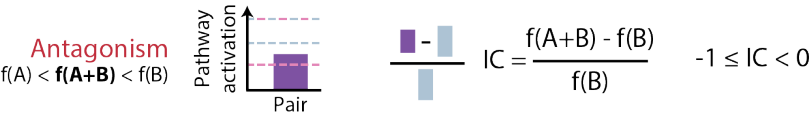

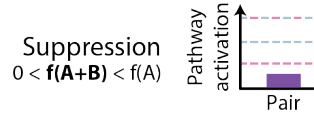

픔 $-2 \quad K=\frac{f(A+B)}{f(A)}-2 \quad I C<-1$

C

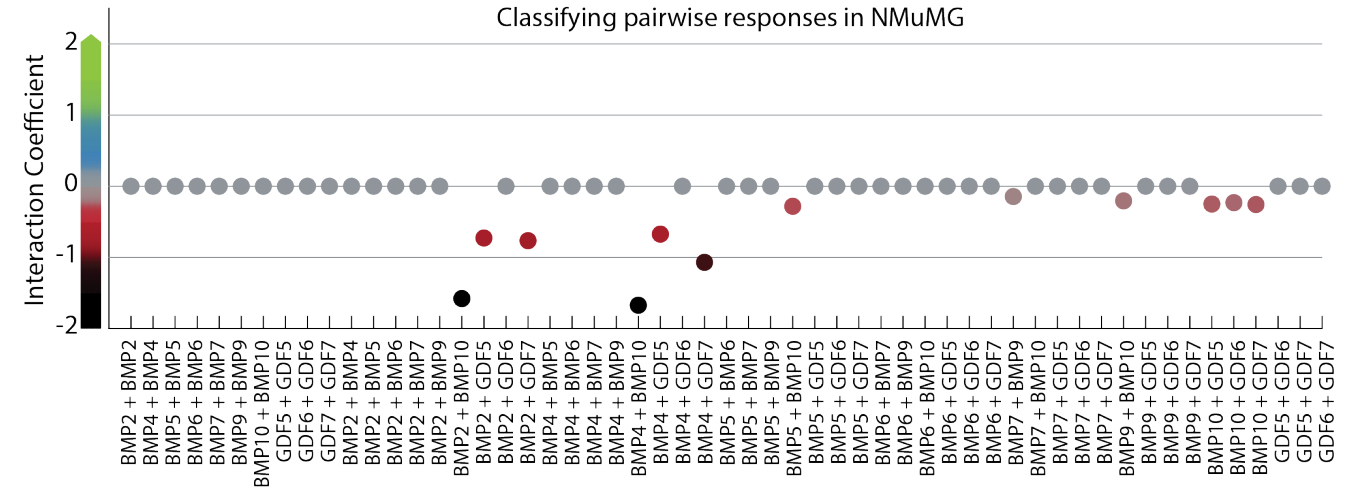

IC Color Scale
B Classifying simulated pairwise responses $10.5 \quad 0$
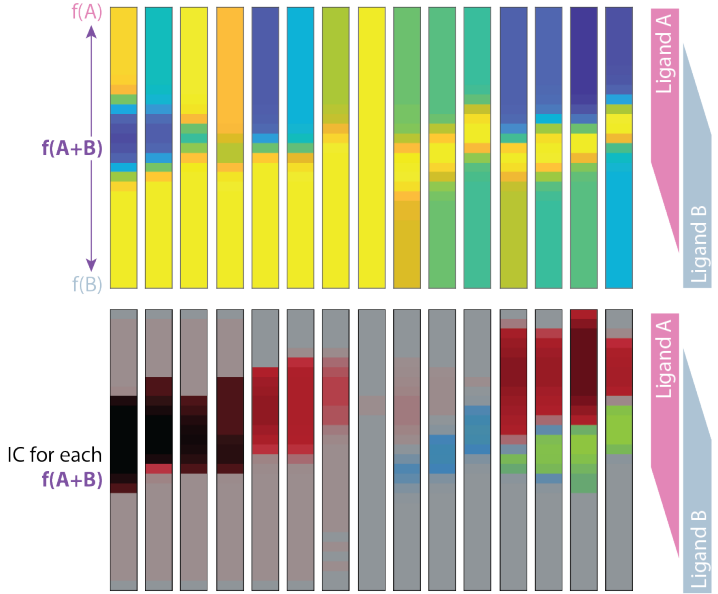

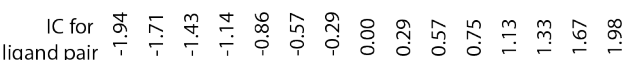

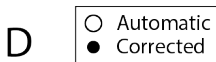

$\mathrm{E}$ Outliers obscure qualitative differences

$\mathrm{F}$
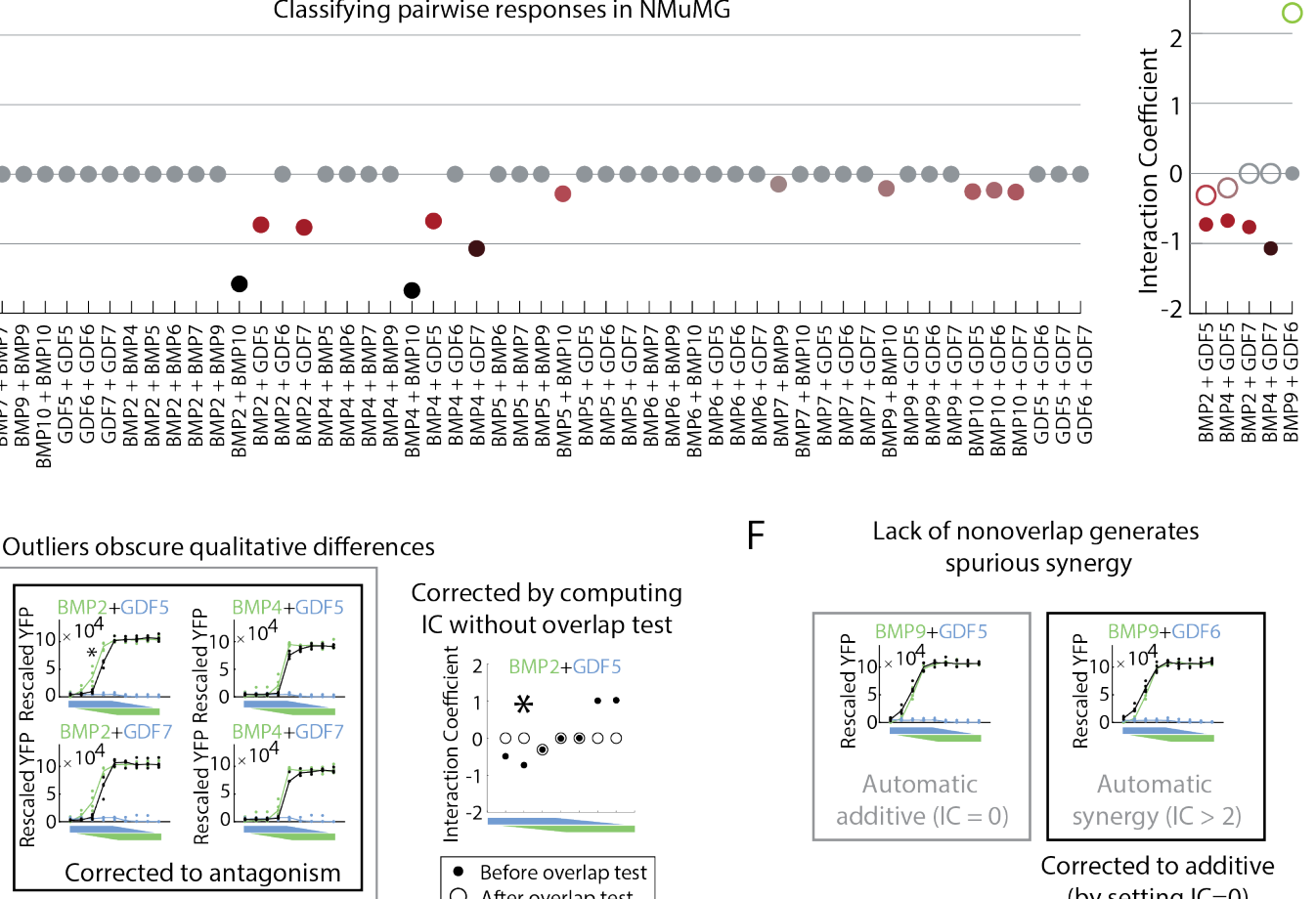

Corrected by computing

IC without overlap test
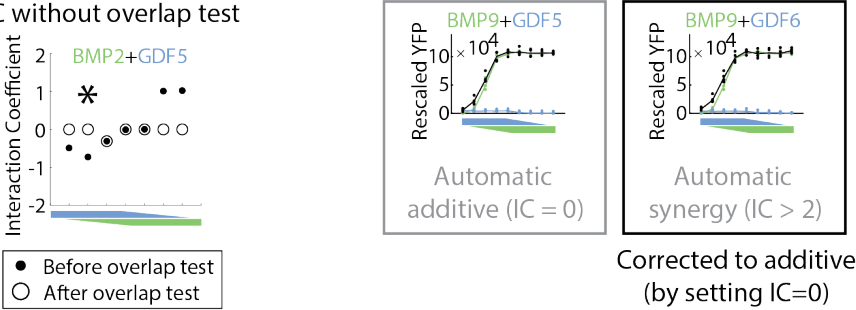

Corrected to additive (by setting $\mathrm{IC}=0$ )

Figure S2.2: Interaction Coefficient (IC) classifies the range of pairwise interactions observed in simulations and experiments. (caption on following page) 
Figure S2.2 (previous page):

(A)The Interaction Coefficient (IC) is computed by a discontinuous, piecewise formula corresponding to four regimes of possible pairwise response. These four regimes (synergy, additivity, antagonism, suppression) are defined by the magnitude of pairwise response relative to the individual responses (i.e. $\mathrm{f}(\mathrm{A}+\mathrm{B})$ relative to $\mathrm{f}(\mathrm{A})$ and $\mathrm{f}(\mathrm{B})$ ).

(B) Variety of theoretical measurements produced by a mathematical model of BMP signaling can be ordered and classified by IC. Top row shows theoretical pathway activation by ligand ratios ranging from pure Ligand A to pure Ligand B. Colors indicate low (blue) and high (yellow) values. Bottom row shows corresponding IC values for each theoretical observation, colored by the IC color scale. Theoretical rims are ordered from largest negative interaction to largest positive interaction.

(C) For each ligand pair, one IC value is computed (STAR Methods). Most responses are saturated additive (gray), with limited instances of antagonism (red) with GDF5 and GDF7 and suppression (black) with BMP10.

(D) Some rims required a different calculation of IC. The automated (open circle) and corrected (closed circle) values are shown for the five pairs where these changes were made. See STAR Methods for full discussion.

(E) To be classified as antagonistic, the full set of biological repeats for a rim response must not overlap with the distribution of measurements for the stronger individual ligand. However, for four of the corrected rims, single outliers created overlap and obscured clear differences between the rim and gradient. For example, BMP2 combined with GDF6 clearly overlaps with the response to BMP2 alone and is additive, while BMP2 and BMP4 combined with GDF5 and GDF7 appear qualitatively different. Compare inset plots of pairwise responses, each of which includes two single ligand responses (plotted in blue and green) and the response to the pair (plotted in black). The x-axis corresponds to multiple ratios of the two ligands, as in A. To correctly classify these rims, the requirement for nonoverlap was neglected. As an example, the IC value for each combination of BMP2 with GDF5 is shown, where the ratio with the strongest antagonism (marked by an asterisk) can be recovered by ignoring the overlap test.

(F) Similarly, nonoverlap between the spread of points for rim and gradient measurements spuriously classifies rims as synergistic, even though very similar looking rims show significant overlap. BMP9 combines with GDF5 and GDF6 in qualitatively similar ways, despite small differences in whether every point on the rim overlaps with the BMP9 gradient. Thus, BMP9 with GDF6 was classified as additive, to match BMP9 with GDF5.

See also Figure 2.3. 


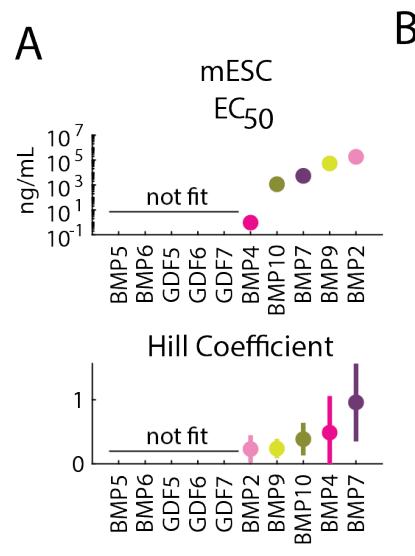

B

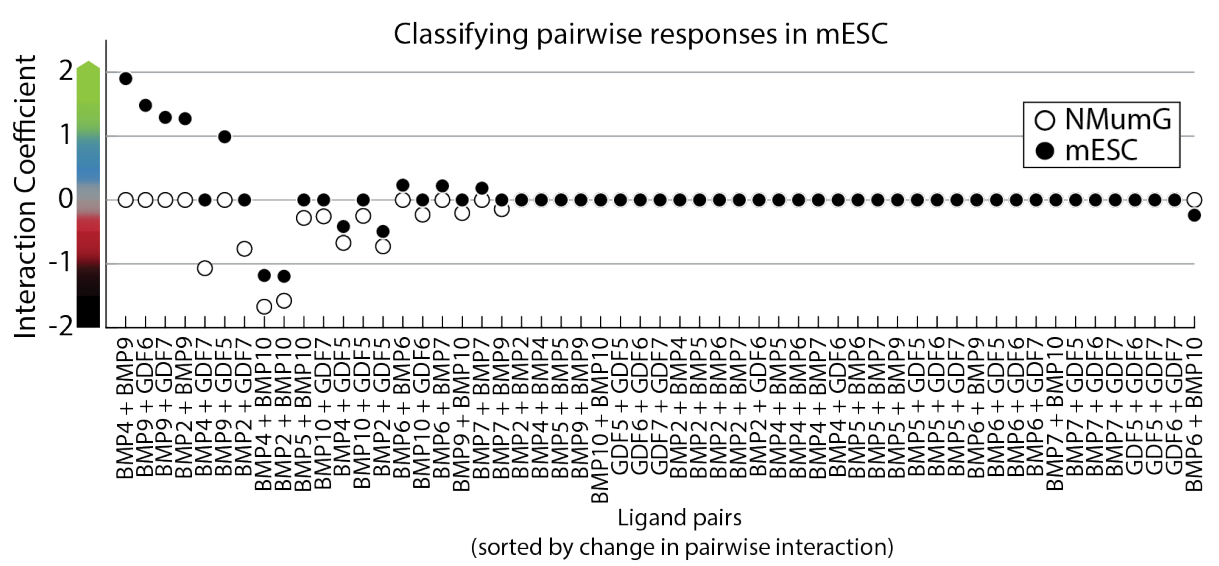

C

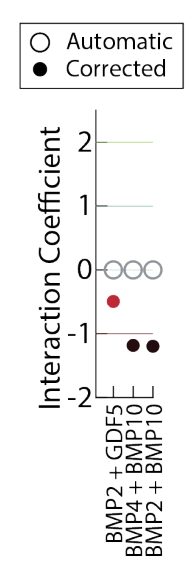

D

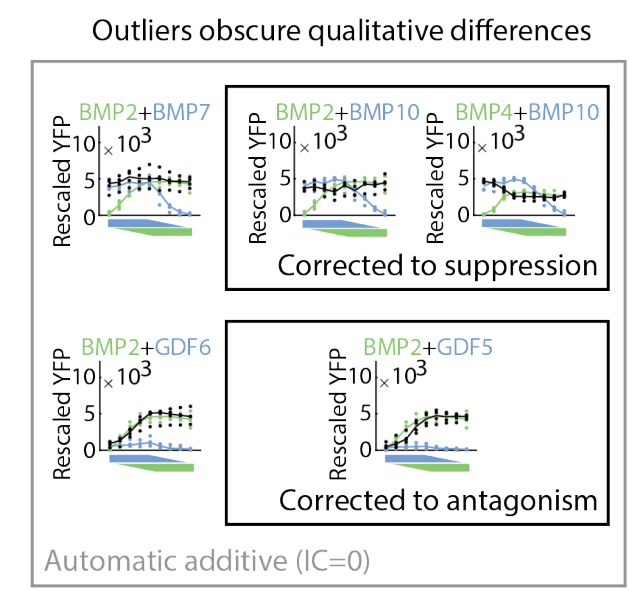

$\mathrm{E}$

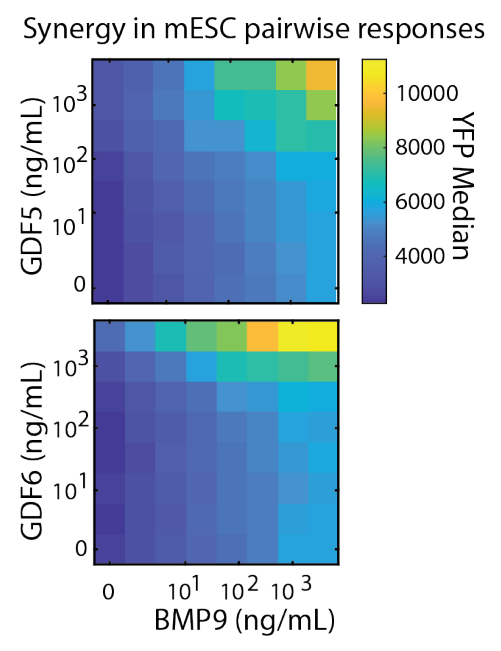

Figure S2.3: Full quantification of BMP signaling in mESC reveals unique ligand properties. (caption on following page) 
Figure S2.3 (previous page):

(A) Dose responses to individual BMPs in mESCs were fit to Hill functions to determine best fit parameters and compare ligand behavior. Ligands that do not activate (GDF5, GDF6, GDF7) or do not saturate (BMP5, BMP6) were not fit. Relative to NMuMG cells, Hill coefficients are much lower for mESCs, and EC50 values are much higher.

(B) Between NMuMG cells and mESCs, a small percentage of pairwise interactions changed significantly, and most changes represented an increase in IC. IC values for all ligand pairs activating mESC (closed circle) and NMuMG cells (open circle, as in S2B) are sorted by decreasing change in IC values.

(C) Automatic classification of pairwise interactions failed for three rims, where antagonistic and suppressive interactions were misclassified as additive. See STAR Methods for full discussion. (D) As in Figure S2.2D, single outliers created overlap between rims and gradients. The corrected IC was computed by ignoring the overlap test for these rims. To show differences between correctly and incorrectly classified pairs, additive interactions of BMP2 with BMP7 or GDF6 are contrasted to BMP2 with BMP10 and GDF5, as well as BMP4 with BMP10.

(E) High-resolution matrices of BMP9 with GDF5 and GDF6 in mESC confirm synergy of BMP9 with non-activating ligands.

See also Figure 2.4 . 

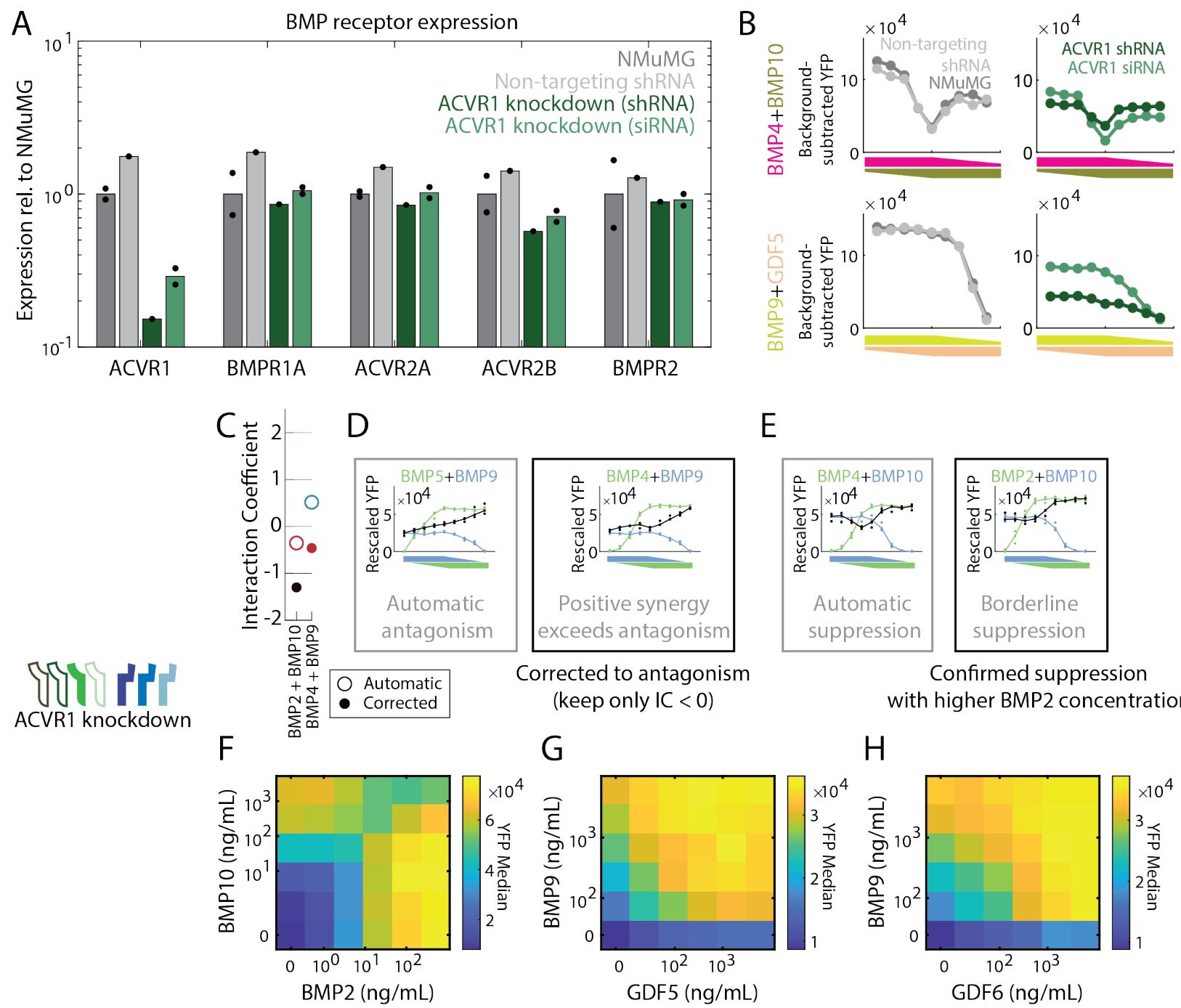

E

G

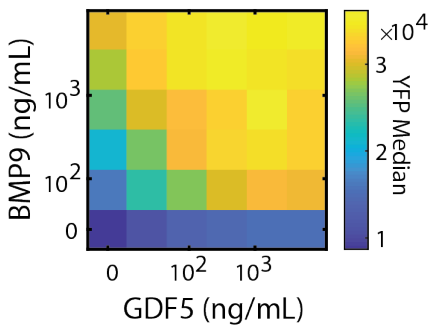

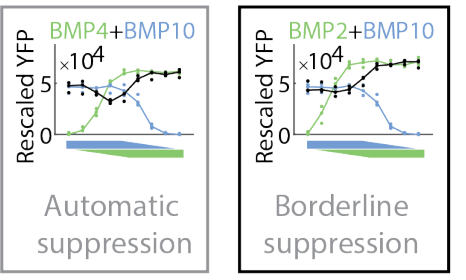

Confirmed suppression with higher BMP2 concentration $\mathrm{H}$

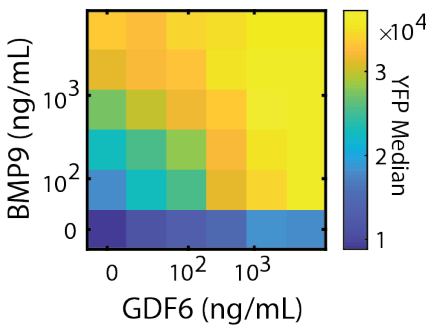

Figure S2.4: Knockdown of ACVR1 by shRNA phenocopies siRNA knockdown and alters pairwise interactions. (caption on following page) 
Figure S2.4 (previous page):

(A) RT-qPCR of BMP receptor expression shows potency and specificity of shRNA knockdown of ACVR1 in parental NMuMG cells. ACVR1-targeting shRNA, or transiently transfected with ACVR1-targeting siRNA. shRNA knocks down ACVR1 mRNA more potently than and as specifically as siRNA, while non-targeting shRNA appears to affect all receptors equally. Expression values are normalized to housekeeping gene expression, and then to the average expression of the same gene in NMuMG cells. Dots are biological replicates, and bar height is either one biological replicate or geometric mean of two replicates.

(B) Responses to ligand pairs sampled at multiple ratios and overall high concentration for same cell types as in A show that non-targeting shRNA has no effect on pairwise responses, whereas knocking down ACVR1 by shRNA or siRNA produces similar effects, with lower activation by BMP4 and BMP9.

(C) Automatic classification of pairwise interactions failed for two rims, as shown in D and E. See STAR Methods for full discussion.

(D) BMP4 combined with BMP9 produced a response with synergistic and antagonistic qualities. However, the synergy is a small effect relative to the antagonism, and the rim more closely resembles another strong antagonistic interaction, BMP5 with BMP9. The corrected classification therefore keeps the IC values corresponding to antagonism.

(E) BMP2 with BMP10 appeared nearly suppressive, mirroring BMP4 with BMP10. Measuring pairwise interactions at a higher BMP2 concentration revealed a strong suppressive interaction (F), where heatmap intensity indicates pathway activation, reported by YFP median. Similarly, sampling responses to BMP9 with GDF5 $(\mathrm{G})$ or with GDF6 $(\mathrm{H})$ confirmed the balance interactions between these ligands. $\mathrm{X}$ - and $\mathrm{y}$-axes in $\mathrm{F}-\mathrm{H}$ correspond to logarithmically increasing ligand concentration, and colors show pathway activation as reported by YFP median.

See also Figure 2.5 . 
A

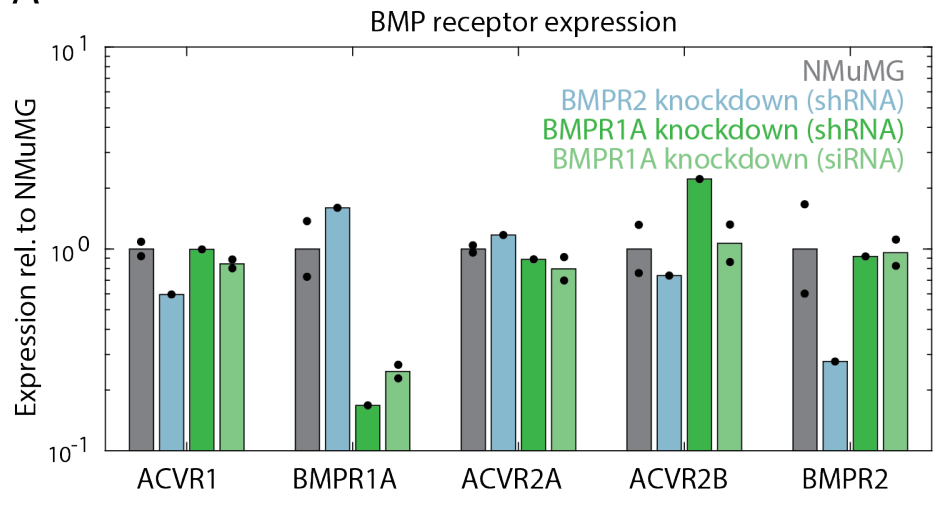

C
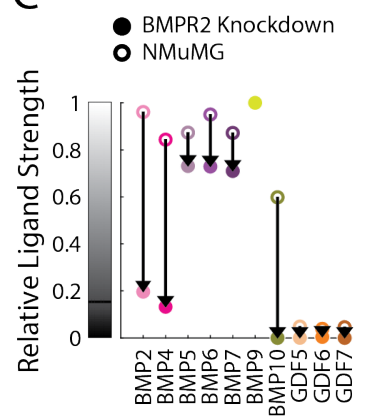

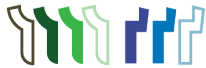

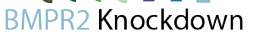

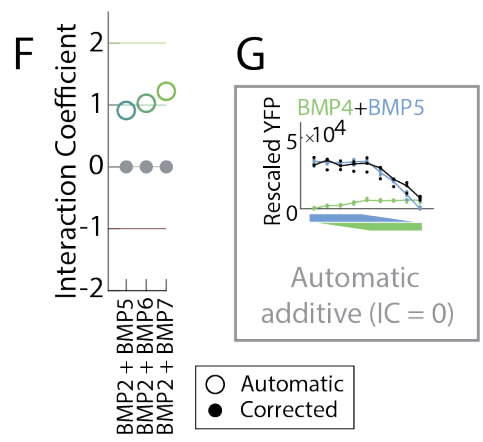

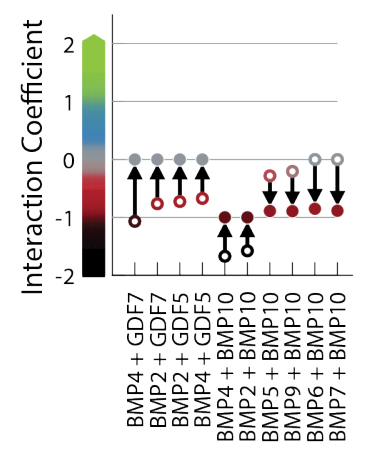

B
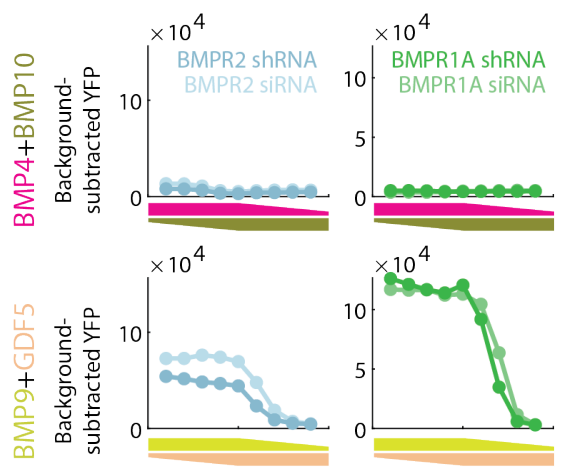

$\mathrm{E}$

IC / RLS Clustering
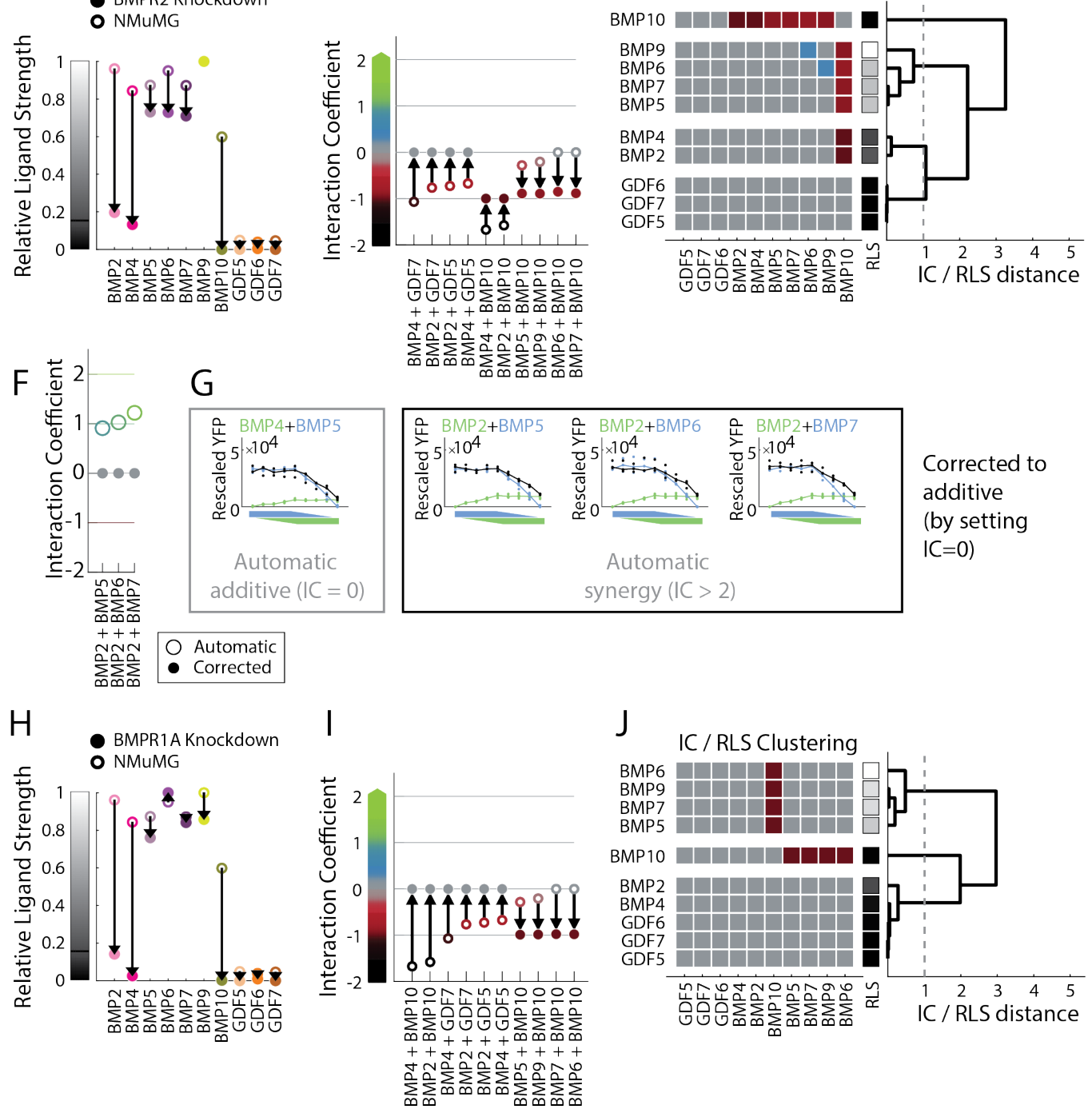

Figure S2.5: Constitutive shRNA expression knocks down BMPR2 and BMPR1A to produce receptor-specific effects distinct from ACVR1 knockdown. (caption on following page) 
Figure S2.5 (previous page):

(A) RT-qPCR of BMP receptor expression shows that constitutively expressed shRNA knocks down BMPR2 and BMPR1A potently and relatively specifically, though with small possible increases in BMPR1A and ACVR2B, respectively. Expression values are normalized to housekeeping gene expression, and then to the average expression of the same gene in NMuMG cells. Dots are biological replicates, and bar height is either one biological replicate or geometric mean of two replicates.

(B) Despite apparent perturbations to off-target BMP receptors, shRNA knockdown produces qualitatively similar responses to ligand pairs as the more specific siRNA knockdown. Pairwise responses are sampled at multiple ratios and overall high concentration in NMuMG reporter with BMPR2 and BMPR1A targeted by siRNA and shRNA.

RLS and IC values change in specific ways following BMPR2 knockdown (closed circle), compared to NMuMG cells (open circle). (C) Activation by BMP2 and BMP4 decreases significantly, while BMP10 activation is lost. (D) Antagonism of BMP2 and BMP4 with GDFs is lost, while all BMP10 interactions become strong antagonism. Changes of IC less than 0.5 are not shown. (E) Hierarchical clustering of all IC and RLS values reveals equivalence groups following BMPR2 knockdown. (F) Automatic classification of pairwise responses failed for three ligand pairs in the BMPR2 knockdown dataset, as shown by the automatic (open circle) and corrected (closed circle) measurements. (G) Small nonoverlap between the rim and the gradient is classified as a synergistic interaction, analogous to Figure $\mathrm{S} 2.2 \mathrm{E}$. The IC values for BMP2 combined BMP5, BMP6, and BMP7 are corrected to 0, to match the qualitatively similar pair, BMP4 with BMP5.

RLS and IC also change following BMPR1A knockdown (closed circle) relative to NMuMG cells (open circle). (H) Activation by BMP2 is decreased while activation by BMP4 or BMP10 is lost. (I) All interactions of BMP2 and BMP4 with other non-activating ligands become additive, while BMP10 strongly antagonizes all activating ligands. Changes of IC less than 0.5 are not shown. (J) Hierarchical clustering of all IC and RLS values reveals equivalence groups following BMPR1A knockdown.

See also Figure 2.6. 

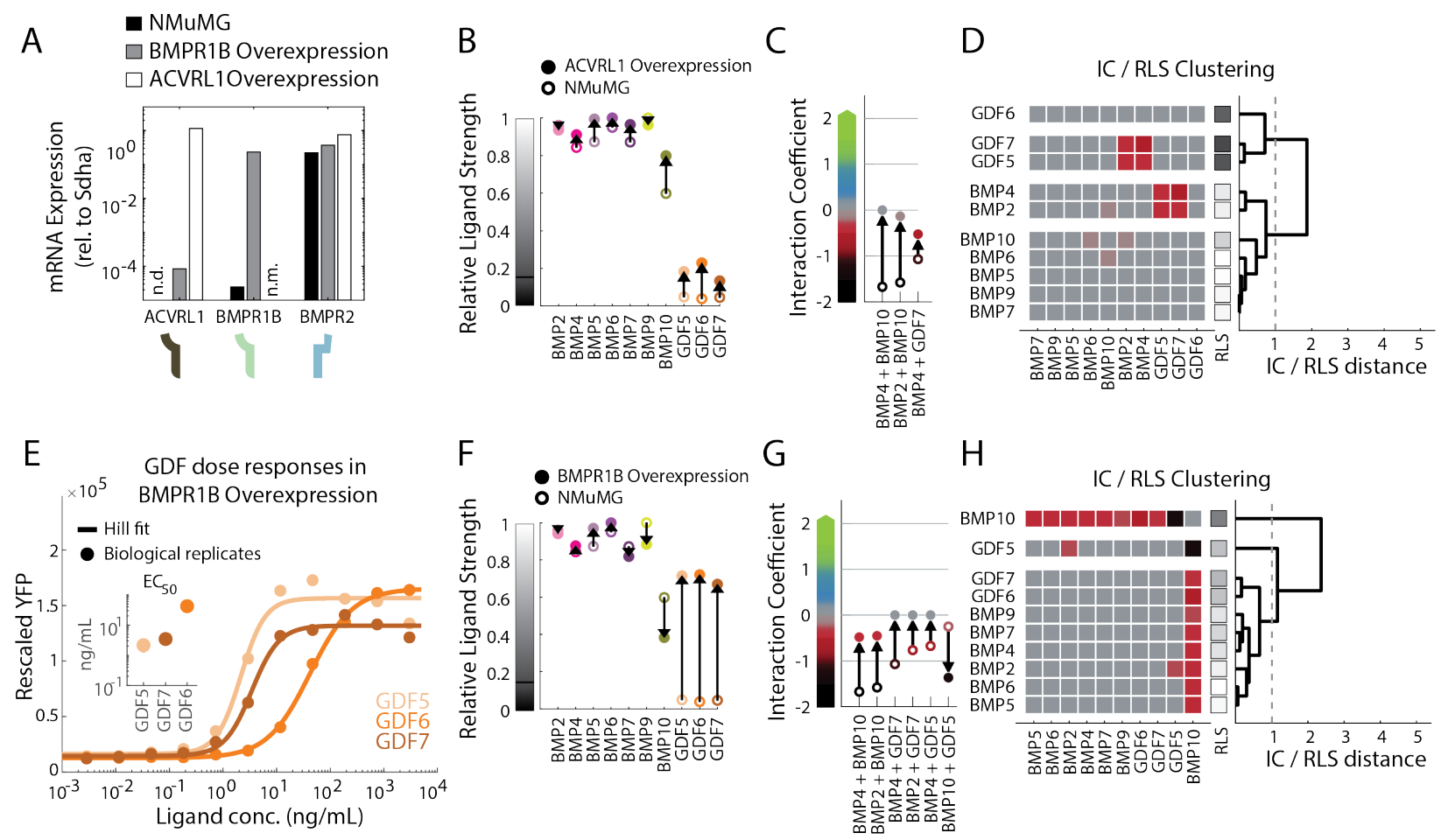

Figure S2.6: Ectopic expression of missing BMP receptors increases frequency of additive interactions and simplifies ligand equivalence. (caption on following page) 
Figure S2.6 (previous page):

(A) ACVRL1 and BMPR1B mRNA are not detected (n.d.) or orders of magnitude lower than housekeeping genes in the original NMuMG reporter. Ectopic expression of ACVRL1 and BMPR1B in the NMuMG reporter increases mRNA levels, quantified by RT-qCPR, to match already expressed BMP receptors, such as BMPR2. BMPR1B levels were not measured (n.m.) in cells ectopically expressing ACVRL1.

(B) Ectopic ACVRL1 expression (closed circle) in NMuMG cells (open circle) produces small changes in RLS, increasing activation by BMP10, and making GDFs weak activators.

(C) Between ectopic ACVRL1 expression (closed circle) and NMuMG cells (open circle), only three ligand pairs show changes in IC larger than 0.5. BMP10's suppression is lost, while GDF7 more weakly antagonizes BMP4.

(D) Hierarchical clustering of all IC and RLS values reveals equivalence groups following ectopic ACVRL1 expression.

(E) GDF5, GDF6, and GDF7 activate NMuMG cells following ectopic expression of BMPR1B, though with different EC50 values (inset, Hill fit parameters) that are not apparent in other cell lines where GDFs do not activate.

(F) Ectopic BMPR1B expression (closed circle) in NMuMG cells (open circle) produces large changes in RLS, converting GDFs into strong activators and slightly decreasing BMP10's strength. (G) Between ectopic BMPR1B expression (closed circle) and NMuMG cells (open circle), six ligand pairs show changes in IC larger than 0.5, and these pairs involve ligands whose RLS changed significantly. BMP10's suppression becomes antagonism, but GDF5, rather than antagonizing BMP10, suppresses it. Otherwise, antagonism by GDFs becomes mostly additive.

(H) Hierarchical clustering of all IC and RLS values reveals equivalence groups following ectopic ACVRL1 expression.

See also Figure 2.6. 

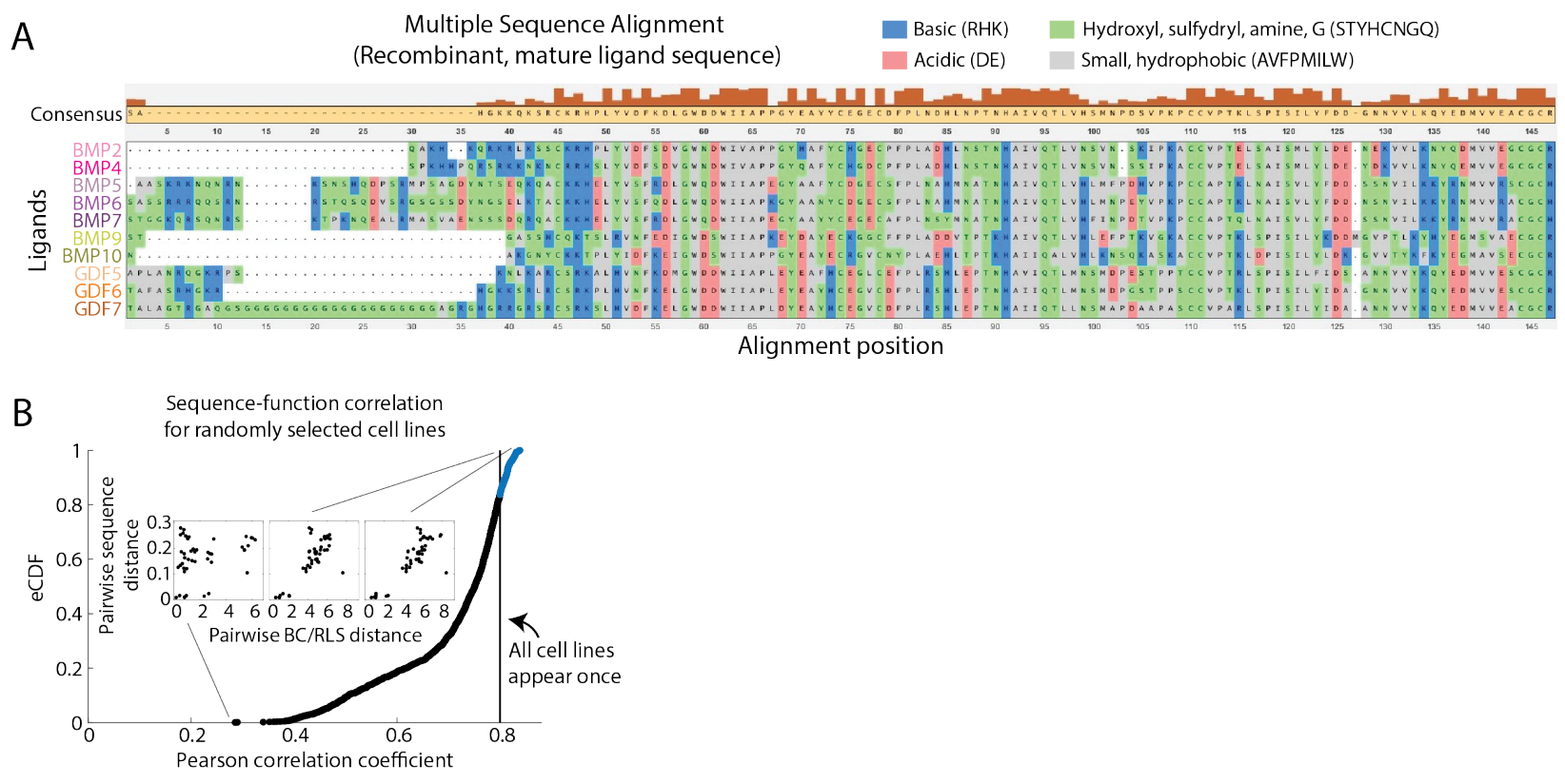

Figure S2.7: BMP ligand sequence differences correlate with global functional differences. (A)Alignment of recombinant BMP sequences reveals amino acid variations between BMP ligands.

(B) The correlation of sequence differences with functional differences was computed for 10,000 random combinations of the seven cell lines. Less than $25 \%$ of samples produce higher correlation coefficients than merely including each cell line once. The best and worst correlations, as well as the correlation for each cell line appearing once, are shown in the inset.

See also Figure 2.6 


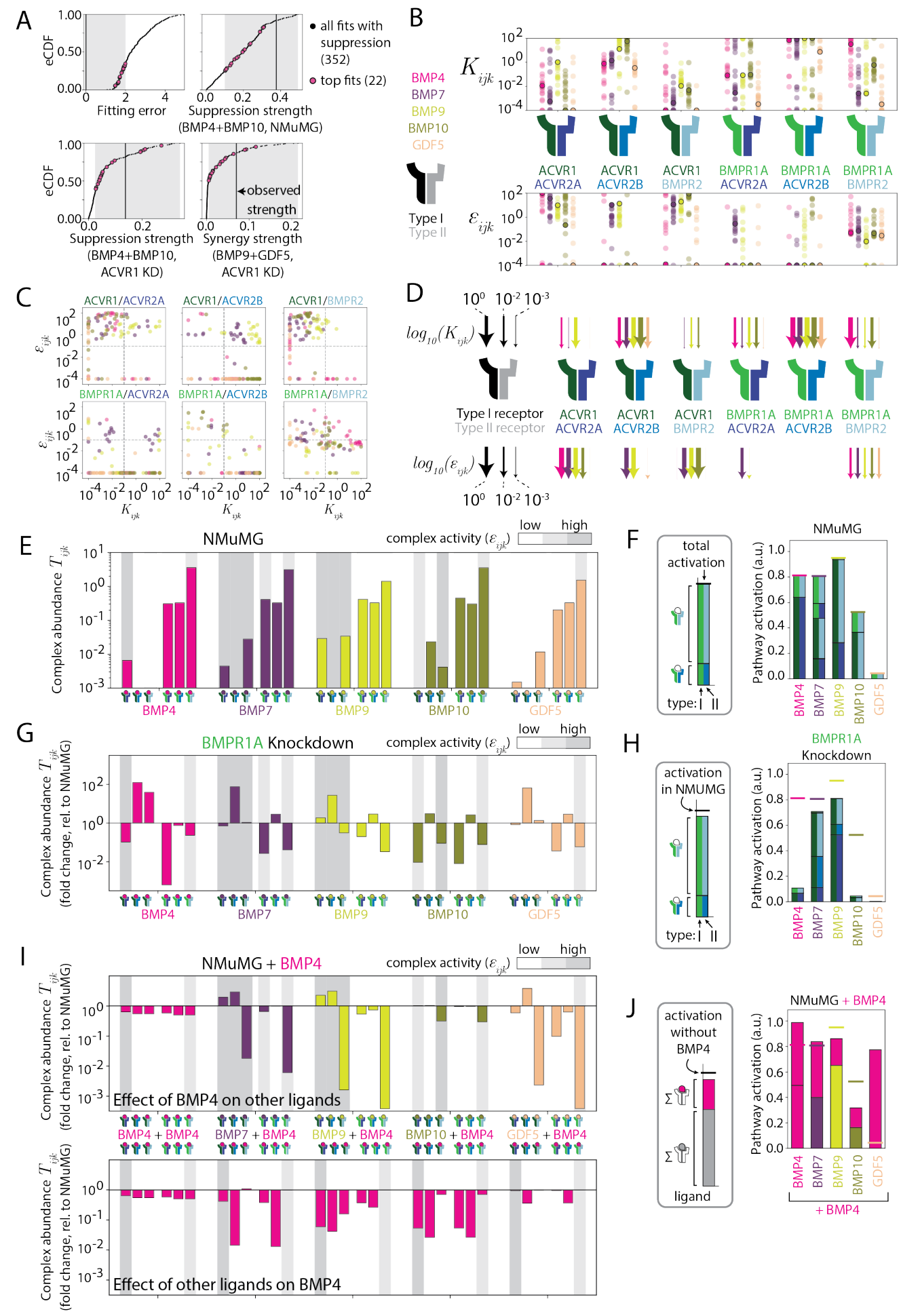

Figure S2.8: In the model, abundance of high- and low-activity complexes depend on affinities, receptor context, and ligand context. (caption on following page) 
Figure S2.8 (previous page):

(A) Of the 6,920 least-squares fits generated, only 352 had fitting error less than 5 while also maintaining the two suppression and synergy interactions observed in the NMuMG and ACVR1 knockdown cells. From these, we selected a top 22 fits which had low error and sufficiently high strength of suppression and synergy. eCDFs for fitting error, suppression strength, and synergy strength are plotted for the 352 solutions which maintained these interactions. The black line shows the observed value, while the gray region shows the accepted regimes for top fits. The pink dots show the top 22 fits which met all four criteria simultaneously.

(B) In the top 22 fits, affinity $\left(K_{i j k}\right.$, upper plot) and activity $\left(\epsilon_{i j k}\right.$, lower plot) parameters varied significantly over the allowed range in many cases, such as BMP4's affinity for BMPR1A-BMPR2. Others were tightly clustered across all parameter sets, such as BMP9's affinity for ACVR1-BMPR2. The parameter fit shown in Figure 2.7B is highlighted with black circles.

(C) Affinity and activity are anti-correlated across all top fits for certain receptor dimers and ligands, such as ACVR1-BMPR2.

(D) A sample parameter set that fits all responses to five ligands in four cell lines are shown as lines for each receptor dimer, where color indicates the ligand species and thickness corresponds to the logarithm of the parameter value. Ligands bind most receptor dimers but activate few. In many cases, affinity and activity are inversely related, as indicated by thick arrows above and thin arrows below, or vice versa.

(E) Abundance of each signaling complex $\left(T_{i j k}\right)$ in the mathematical model varies slightly between individual ligands when activating NMuMG cells. The possible complexes are grouped into sets of six, colored by the contained ligand and with the six bars corresponding to the six possible receptor dimers, in the same order as in B and cartooned with the same colors on the x-axis. Grayscale background for each bar indicates the binned (high $>100$, low $<10^{-2}$, medium in between; arbitrary $\epsilon_{i j k}$ units) activity of that complex. Ligands form similar amounts of the various complexes, but differ in their ability to activate them.

(F) Strongly activating ligands achieve similar levels of pathway activation by activating distinct, ligand-specific receptor dimers, whereas weaker ligands activate fewer receptor dimers. Stacked bars show the contribution of various signaling complexes. Left and right halves of each unique bar in the stack are colored to indicate unique pairings of the Type I and Type II receptors, respectively.

(G) Bars indicate the fold change, relative to NMuMG cells, in signaling complex abundance following BMPR1A knockdown. Ordering of bars and grayscale background are as in E. BMPR1A knockdown indirectly increases abundance of ACVR2B-containing complexes, which only BMP7 and BMP9 activate strongly.

(H) As in D, individual ligands activating the BMPR1A knockdown cell line at saturating concentrations utilize different receptor complexes. Unexpectedly, BMP4 and BMP10 produce lower activity overall, due to the loss of ACVR1-containing complexes that produced most of their pathway activation, while BMP7 and BMP9 shift to other ACVR1-containing complexes.

(I) The presence of saturating BMP4 introduces additional, BMP4-containing complexes and changes complex abundance as receptors are shared between the two ligands. Bars indicate the theoretical fold change, relative to ligands signaling alone, in the amount of each receptor complex. For each ligand pair, the full set of complexes are split between the upper plot (the first ligand of the pair) and lower plot (BMP4, the second ligand of the pair). Ordering of bars and grayscale background are as in E. BMP7 and BMP9 lose some activating complexes to BMP4 while also forming new ones, due to their ability to promiscuously activate all ACVR1-containing complexes.

(J) Bar height indicates the theoretical contribution of each ligand to overall activation by the ligand pair. Consistent with the effects of competition, overall pathway activation is reduced when BMP4 is combined with BMP10, increased when combined with GDF5, but stays the same, due to sharing of active complexes, when combined with BMP7 and BMP9.

See also Figure 2.7

\subsection{STAR Methods}

\subsubsection{Resource Availability}

\section{Lead Contact}

All requests for resources and reagents should be directed to and will be fulfilled by the Lead Contact, Michael Elowitz (melowitz@ caltech.edu).

\section{Materials Availability}

The recombinant proteins, lentiviral particles, siRNAs, and qPCR probes used in this paper are described in Tables tables 2.2 to 2.5 and can be purchased from commercial sources, as detailed 
in the Table 2.1. Plasmids and cell lines generated for this paper, as indicated in Table 2.1, are available from the Lead Contact upon request.

\section{Data and Code availability}

The datasets and analysis scripts generated used to produce paper figures are available here: https://doi.org/10.22002/D1.1693.

\subsubsection{Experimental Model and Subject Details}

Tissue culture and cell lines

NMuMG (NAMRU Mouse Mammary Gland cells, female) were acquired from ATCC (CRL1636). E14 cells (mouse embryonic stem cells, E14Tg2a.4, male) were obtained from Bill Skarnes and Peri Tate. All cells were cultured in a humidity-controlled chamber at $37^{\circ} \mathrm{C}$ with $5 \% \mathrm{CO}_{2}$. NMuMG cells were cultured in DMEM supplemented with 10\% FBS (VWR \#311K18), 1mM sodium pyruvate, 1 unit $/ \mathrm{mL}$ penicillin, $1 \mu \mathrm{g} / \mathrm{mL}$ streptomycin, $2 \mathrm{mM} \mathrm{L}$-glutamine, and $1 \mathrm{X}$ MEM non-essential amino acids. ES cells were plated on tissue culture plates pre-coated with $0.1 \%$ gelatin and cultured in standard pluripotency-maintaining conditions ${ }^{66}$ using DMEM supplemented with 15\% FBS (ES qualified, GIBCO \#16141), 1mM sodium pyruvate, 1 unit/mL penicillin, $1 \mu \mathrm{g} / \mathrm{mL}$ streptomycin, 2mM L-glutamine, 1X MEM non-essential amino acids, $55 \mu \mathrm{M} \beta$-mercaptoethanol, and 1000 units/mL leukemia inhibitory factor (LIF).

\section{Reporter cell line construction}

To construct reporter cell lines, a plasmid harboring the BMP response element ${ }^{39} \mathrm{i} n$ the enhancer region of a Major Late Promoter (MLP) driving the expression of an H2B-mCitrine protein fusion was integrated into the NMuMG genome using PiggyBac integration (System Biosciences). After transfection, cells were selected with $100 \mu \mathrm{g} / \mathrm{mL}$ hygromycin. The base NMuMG reporter was a clonal cell line generated by limiting dilution. This reporter construct exhibited lower background YFP expression and increased signaling dynamic range relative to a similar NMuMG transcriptional reporter, ${ }^{[4}$ consistent with other observations of MLP's lower background relative to mCMV ${ }^{[23} \mathrm{A}$ previously-reported mESC reporter was used. ${ }^{4}$ To construct this reporter, a related construct, carrying minimal CMV in place of MLP, was randomly integrated into the E14 genome using the FugeneHD reagent and a clonal population selected by colony picking. 


\subsubsection{Method Details}

\section{Quantifying BMP responses with flow cytometry}

To assay BMP pathway activity, we genomically integrated a transcriptional reporter into all cell lines as described above (see "Reporter cell line construction"), which expressed YFP proportional to pSMAD activation. ${ }^{4}$ To measure how a given ligand or combination of ligands activated the pathway, we followed the following protocol in all robotic or manual experiments. Cells were plated at a confluency that, assuming a 24 hour doubling time, would produce sufficient cells for flow cytometry at the experiment endpoint (i.e. at least 5000 cells 36 hours after plating in a 96 well format, or about $20-30 \%$ confluent at the start of the experiment). Twelve hours after plating, cell media was removed and replaced with ligand-containing media. Twenty-four hours after ligands were added, cells were lifted from the plate for flow cytometry, by five minute incubation at $37^{\circ} \mathrm{C}$ in $0.25 \%$ Trypsin (or Accutase, for mESCs) following PBS wash. Trypsinization was quenched by resuspending cells in flow buffer, (HBSS containing $2.5 \mathrm{mg} / \mathrm{mL}$ Bovine Serum Albumin (BSA)). Cells were then filtered through a $40 \mu \mathrm{m}$ mesh and analyzed by flow cytometry (MACSQuant VYB, Miltenyi or CytoFLEX, Beckman Coulter). Note that because of difficulties with cellular aggregation following filtering, alternative flow buffer formulations additionally included either $1 \mathrm{mM}$ ethylenediaminetetraacetic acid (EDTA) or 200U/mL DNAse I in some cases.

\section{Robotic liquid-handling protocol}

For each cell line, a similar protocol was used to generate a full pairwise dataset. First, the transcriptional BMP reporter was generated, and the receptor profile was assayed by qPCR. The only exception was the mESC reporter, whose receptor profile was obtained from a prior publication that assayed receptor expression by RNA-Seq. ${ }^{4}$ Then, we determined the dynamic range of each ligand in this new receptor context. To do this, we completed at least one biological replicate of single ligand dose responses up to very high concentrations that were expected to approach saturation (e.g. $3 \mu \mathrm{g} / \mathrm{mL}$ ), with a high fold dilution to capture the full dynamic range. These dose responses fell into three types: saturating activators, non-saturating activators, and nonactivators. For saturating activators, we selected a maximum concentration and fold dilution that sampled concentrations corresponding roughly to $25,50,75$, and $100 \%$ of maximal activation. For non-saturating activators, maximal activation was unknown. To avoid supraphysiological concentrations, we limited the maximum concentration to 2 or $3 \mu \mathrm{g} / \mathrm{mL}$ and used a minimum 2-fold dilution to sample what little of the dynamic range was accessible. For non-activators, there was no dynamic range. We therefore selected maximum concentrations and fold dilutions that approximated the median dynamic range of the activating ligands, reasoning that these highly 
homologous ligands had, to a first approximation, reasonably similar concentrations over which they were most active. The full list of all concentrations and fold dilutions used for each cell line is in Table 2.2. Next, we assayed all pairwise interactions, with single ligand gradients as well as "pseudo-pairs" of ligands with themselves as controls. For ten ligands, the screen included 10 gradients, 10 "psuedo-pairs," and the 45 possible pairs, or 65 rows of measurements. Each row included a no BMP control, plus the nine ratios at which each pair (ligand by itself, ligand paired with itself, or ligand paired with another ligand) was assessed.The order of the rows and of the ligands was scrambled between biological repeats. To expose cells to these complex ligand combinations, we used a custom liquid-handling protocol on the Tecan EVO Freedom 200 base unit, which was housed in a sterile, laminar flow hood. To start the experiment, we manually plated cells eight to twelve hours before the start of the robotic protocol and prepared serial dilutions of the ten ligands by hand. The robotic protocol then added the precise ligand combinations to the appropriate plates, and timed the start of each plate to match the staggered analysis of each plate twenty-four hours after the ligand combinations were added. Specifically, at the appropriate time point, the robot removed a plate from the incubator, aspirated the media, replaced it with the appropriate ligand-containing media, and returned cells to the incubator. The robot incubator was fed $5 \% \mathrm{CO}_{2}$. All plates were then collected from the robot incubator at the end of the experiment. Finally, we quantified cell responses in each of these ligand conditions. 20 to 24 hours after the BMP ligands were added, we measured YFP of each well by flow cytometry, as described above (see "Quantifying BMP reporter with flow cytometry"). We analyzed the resulting flow data with custom MATLAB scripts available at https://doi.org/10.22002/D1.1693, following the steps described below in "Data Processing."

\section{Stable shRNA knockdown and ectopic receptor expression}

Individual BMP receptors were knocked down or ectopically expressed in the base NMuMG reporter cell line. For receptor knockdown, lentiviral particles containing constructs for constitutive shRNA expression reported by mCherry (SMARTvector, Dharmacon) were transduced into cells. Per manufacturer's instructions for determining optimal transduction conditions, we transduced cells at an MOI of 20, using serum-free NMuMG growth media supplemented with $10 \mu \mathrm{g} / \mathrm{mL}$ polybrene. $48 \mathrm{~h}$ after transduction, cells were selected and continuously maintained in $3 \mu \mathrm{g} / \mathrm{mL}$ puromycin. Cells with BMPR1A and BMPR2 knockdowns were polyclonal populations generated by a single shRNA. Cells with ACVR1 knockdown were a clonal population selected by limiting dilution of cells transduced with a pool of three shRNAs. For a list of the shRNAs used, see Table 2.3. For ectopic expression of BMP receptors, constructs for constitutive expression of mouse receptor cDNAs, reported by mTurquoise, were integrated into the base NMuMG reporter by PiggyBac 
integration (Systems Bioscience), using previously-reported plasmids (Antebi et al., 2017). Cells were selected and maintained with $500 \mu \mathrm{g} / \mathrm{mL}$ geneticin.

\section{Transient siRNA knockdown}

Cells were plated at $40 \%$ confluency in a 24 well plate format with $30 \mu \mathrm{M}$ total siRNA (ThermoFisher Silencer Select \#4390771) and 3 $\mu$ L RNAiMAX (Life Technologies). For every gene, a pool of two distinct siRNAs was used, listed in Table 2.4. Cells were passaged after $24 \mathrm{hr}$ and then used for the relevant experiments.

\section{RT-qPCR}

Total RNA was harvested from cell lysate using the RNeasy mini kit (QIAGEN), and cDNA was generated from $1 \mu \mathrm{g}$ of RNA using the iScript cDNA synthesis kit (BioRad) per the manufacturer's instructions. Primers and probes for specific genes (Table 2.5) were purchased from IDT. Reactions were performed using 1:40 dilution of the cDNA synthesis product with either IQ SYBR Green Supermix or SsoAdvanced Universal probes Supermix (BioRad). Cycling was carried out on a BioRad CFX96 thermocycler using an initial denaturing incubation of $95^{\circ} \mathrm{C}$ for $3 \mathrm{~min}$ followed by 39 cycles of $\left(95^{\circ} \mathrm{C}\right.$ for $15 \mathrm{~s}$, followed by $60^{\circ} \mathrm{C}$ for $\left.30 \mathrm{~s}\right)$. Each reaction was run at least in duplicate.

\subsubsection{Quantification and Statistical Analysis}

\section{Analysis of RNA-Seq datasets}

To survey BMP ligand and receptor expression across various tissues, we selected relevant datasets from a collection of bulk, mouse RNA-Seq datasets. ${ }^{40}$ Datasets that indicated the sample library contained transcriptomic cDNA prepared from polyA RNA and that indicated a tissue-related source were kept. RNA-Seq quantification of BMP receptor expression in the NMuMG parental cell line and the mESC reporter cell line (cf. Figure $\mathbf{S 2 . 3 A}$ ) was taken from GSE98674.

\section{BMP alignment and clustering}

To analyze amino acid sequences of BMP ligands, the relevant recombinant sequences (indicated by R\&D Biosystems) were globally aligned with MATLAB's multialign function, using default parameters. Distance between sequences was computed by the BLOSUM50 matrix, comparing only positions with no gaps across all aligned ligands, and they were clustered with average-distance linkage. 


\section{Data processing}

All flow cytometry data for gradients and rims was gathered by the same method, implemented manually or robotically (see "Quantifying BMP reporter" and "Robotic liquid-handling protocol" above). The resulting flow data were analyzed in the following manner. Using a custom MATLAB GUI (https: //antebilab.github . io/easyflow/), cells were gated on Forward Scatter (FSC) and Side Scatter (SSC), and median YFP used to summarize pathway activation in each well. A custom MATLAB script then dropped any wells with fewer than the threshold number of cells (i.e. 500), reordered wells to match standard ligand order, and subtracted background. For rims, an additional step minimized noise between the eleven plates, which, due to small variation in plate preparation and processing times, had incubation times varying from 20 to 24 hours. Assuming linear accumulation of YFP with time, variation in incubation generates a fold change in background-subtracted YFP. Therefore, each plate was linearly rescaled to a reference plate to minimize total least squares error between technical replicates shared between the two plates. Following standardization of each biological replicate, they were aggregated by another script. To reduce variance due to biological noise, each biological replicate was rescaled by a single linear factor that minimized total least squares error between all corresponding measurements in the two replicates. To complete gradient analysis, all biological replicates were fit to a Hill function by minimizing total least squares error between the Hill fit and all rescaled biological replicates. To complete rim analysis, Relative Ligand Strength (RLS) and Interaction Coefficients (IC) were computed. RLS is the ratio of each ligand's maximal activation to the strongest ligand's maximal activation. However, the IC metric, defined to quantify small differences in pathway activation, required more careful analysis to ensure robustness to technical and biological noise, as described in the following section.

\section{Interaction Coefficient}

\section{Drug synergy classification cannot fully capture ligand interactions}

Classification of synergy and antagonism occurs in many fields, including the study of drug interactions and gene epistasis. $17|43| 56 / 78 \mid 82$ However, these metrics do not have clear analogies for classifying ligand interactions for at least two reasons. First, all classifications of synergy, when agnostic of mechanism, depend on a phenotypic definition of additivity, i.e. a non-interacting relationship. However, the many definitions of additivity developed for drug interactions are not consistent with each other and not necessarily appropriate to describe ligands activating the same pathway. As an example of this, most definitions of additivity descend from approaches defined by 
Loewe $\mathrm{e}^{41}$ and Bliss, 919 who predicted that additive outcomes would be the sum of each component's dose or effect, respectively. ${ }^{56}$ However, these assumptions make little sense in the context of two signaling proteins that use the same signaling components to produce their individual effects; indeed, individually saturating concentrations are not expected to sum in their effects or their normalized dose. A third approach to additivity considers the effect of each drug as an independent event, such that a non-additive response deviates from the known joint probability of independent outcomes ${ }^{78}$ But the sharing of pathway components between the two ligands makes this assumption of independence inappropriate. Second, classifications of interaction type depend on well-defined regimes of behavior. The effect of drug combinations on growth rate has distinct boundaries for these regimes, but there are not clear boundaries for pathway activation. For example, the largest effect of a drug is to decrease growth rate to zero, but the upper bound of a signaling protein's effect could be set by any one of the pathway components being limiting and so depends heavily on cell context. Similarly, drugs can exhibit a "strong" form of antagonism when a combination causes cells to grow faster than in the absence of drugs. By contrast, signaling proteins cannot, in combination, decrease pathway activation below zero, i.e. the response in the absence of signaling proteins. Thus pathway activation requires a different separation between weak and strong antagonism.

\section{Interaction Coefficient captures features specific to ligand interactions}

To address the incongruency of drug and ligand interactions, we developed the Interaction Coefficient, a definition of additivity and associated regimes of behavior that are appropriate for ligand interactions as described above. The goal of defining this metric was to quantify and compare specific ligand behaviors of interest, rather than providing a generic or alternative synergy metric

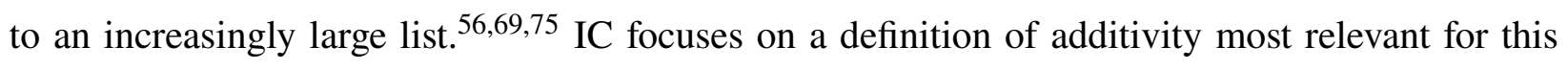
study: additively interacting ligands combine with each other in the same way they combine with themselves. At first, this presents an apparent challenge, as the way a ligand combines with itself is inconsistent. In the limit of very low and very high concentrations, doubling ligand concentration has no effect on pathway output. Conversely, at intermediate concentrations, doubling ligand concentration can increase pathway output by two-fold or more, depending on the cooperativity of the response. Rather than making the strong claim that any one of these behaviors is the true additive expectation, IC includes both as boundaries between distinct behavioral regimes, where output greater than the sum is synergy and output less than the maximum of the two is antagonism of some strength. Finally, rather than predicting which of these two behaviors would result when a ligand was added with itself, we simply measured them. Thus, while ICAB describes the phenotypic response when ligand $\mathrm{A}$ combines with ligand $\mathrm{B}, \triangle \mathrm{IC}$, or the difference between $I C_{A A}$ and $I C_{A B}$, indicates whether any apparent non-additivity between $\mathrm{A}$ and $\mathrm{B}$ is truly unexpected. To complete 
our definition of IC, we add a third boundary dividing weak and strong antagonism, determined by whether the combination response interpolates or is less than both individual responses. Thus, the three boundaries define four behavior regimes. Within each regime, the strength of that behavior type can be computed by normalizing the observed response to the maximal effect size. This generates the following definition of IC:

$$
I C= \begin{cases}\frac{f(A+B)}{f(A)+f(B)} & f(A+B)>f(A)+f(B) \\ \frac{f(A+B)}{[f(A)+f(B)]-f(B)} & f(A)+f(B)>f(A+B)>f(B) \\ \frac{f(A+B)-f(B)}{f(B)-f(A)} & f(A)+f(B)>f(A) \\ \frac{f(A+B)}{f(A)}-2 & f(A)>f(A)+f(B)\end{cases}
$$

where $f(X)$ is the nonnegative pathway response to ligand input $X$, and $f(B)>f(A)$. Note that IC has no upper bound, consistent with the lack of upper bound on pathway activation, and that this calculation assumes all data have been background subtracted. However, this definition of IC misclassifies small absolute changes in $\mathrm{f}(\mathrm{A}+\mathrm{B})$ as strong pairwise interactions in a few cases. Specifically, if $f(A) \approx 0$ or $f(B) \approx f(A)$, the denominator of IC diverges, respectively, in the additive and antagonistic regimes, effectively due to the collapse of one of the behavior regimes. We therefore defined a discontinuous definition of IC, which normalizes each regime to a more intuitive standard that does not approach zero, which is the response to the stronger ligand individually, or $f(B)$. The Interaction Coefficient (IC) as reported in this paper is computed with the following piecewise formula:

$$
I C= \begin{cases}\frac{f(A+B)}{f(A)+f(B)} & f(A+B)>f(A)+f(B) \\ \frac{f(A+B)}{f(B)} & f(A)+f(B)>f(A+B)>f(B) \\ \frac{f(A+B)-f(B)}{f(B)} & f(A)+f(B)>f(A) \\ \frac{f(A+B)}{f(A)} & f(A)-2>f(A)+f(B)\end{cases}
$$

where $f(X)$ is the nonnegative pathway response to ligand input $X$, and $f(B)>f(A)$, as above. To describe the interaction of any ligand pair, the largest magnitude IC value, positive or negative, for all ratios of $A$ to $B$ is reported.

\section{Determining the Interaction Coefficient from noisy data}

Because $f(X)$ is sampled only three or four times from a broad, underlying distribution, we sought a robust metric to determine if two sets of measurements, $f(X)$ and $f(Y)$, could be reasonably 
considered different from each other, in both a statistical and biological sense. Our first criterion was that the range of replicates of $f(X)$ must not overlap with the range of $f(Y)$ replicates. However, even technical replicates collected on different days (i.e. $f(X)$ and $f\left(X^{\prime}\right)$ ) were sometimes nonoverlapping, indicating these measurements were distributed over a large range. Therefore, we added an additional criterion that the degree of non-overlap must exceed a certain threshold. Many of the apparently spurious classifications occurred for low YFP values that were a large percentage change in background but an infinitesimal percentage of the overall dynamic range. Therefore, we set a threshold for effect size, which was the maximum, background-subtracted YFP observed in a given cell line's full dataset divided by 30, i.e. 3\% of the cell line's dynamic range. This threshold achieved two objectives, nearly eliminating spurious identification of strong pairwise interactions due to small variations in background and most closely matching a manual classification of pairwise interaction type. Nonetheless, the correct, automatic classification of some rims was not possible with this approach, perhaps due to a liquid-handling error specific to one or a set of measurements. For these 13 of the 385 total pairs ( $<4 \%$ of the full dataset of 7 cell lines with 45 pairs and 10 controls pairs each), we determined a more reliable estimate of IC value by selecting a less noisy point on the rim, calculating it from independent measurements of that same pair, or setting it to 0 for the null hypothesis of additivity. A full description of these pairs and their manual classification is included below.

\section{Rim classification corrections}

Confident, automatic classification of all pairwise interactions posed a significant challenge. Measurements were limited to three or four biological replicates while small robotic errors, such as dropped tips or cross-well contamination, randomly disturbed a small subset of the data. While the analysis pipeline outlined above minimized effects of technical and biological noise by rescaling, and randomization of experiment layout distributed any systematic errors more evenly, the automatic classification of pairwise interactions did not match our intuition in a small number of cases (13 of the 385 rims). Here we describe those cases, why they resisted automatic classification, and how we manually corrected the classification. First, for two measures of pathway activation to be considered different, the ranges of their respective biological replicates had to be non-overlapping. However, a single outlier among three or four points may cause these ranges to overlap, despite significant differences in the median. Thus, plausible non-additive interactions may be classified as additive by one erroneous measurement. For example, in NMuMG cells, BMP2 and BMP4 combined with GDF5 or GDF7 produced antagonism distinct from GDF6's lack of effect of BMP2 (Figure S2.2D). Similarly, in mESC, BMP2 and BMP4 interacted very differently with BMP10 than with other activating ligands, and BMP2 was antagonized by GDF5, but not GDF6 (Figure 
$\mathrm{S} 2.3 \mathrm{E})$. However, despite these qualitative differences, all these rims were classified as additive due to a single outlier. For correct classification of these rims, the requirement for non-overlap was removed for the specific points on the rim where the pairwise interaction was clear (cf. asterisks in Figure S2.2D). Second, a few rims were classified as strong synergy due to a small increase in pathway activation at subsaturating concentrations, though synergy manifesting exclusively at extreme ligand ratios or small absolute effect sizes, both of which were unlikely for true synergistic interactions. Moreover, given the width of the distribution of median YFP measurements, multiple samples from the same distribution could often, by random chance, produce non-overlapping measurements. For example, In NMuMG cells, despite the similarity of responses to BMP9 paired with GDF5 or GDF6, the latter was classified as synergy due to lack of overlap at low activation levels (Figure $\mathrm{S} 2.2 \mathrm{E}$ ). Similarly, in the BMPR2 knockdown cell line, BMP2 paired with BMP5, BMP6, and BMP7 showed synergy at low activation levels, despite not much clear difference from BMP4's additive interaction with BMP5 (Figure $\mathrm{S} 2.5 \mathrm{G}$ ). These small variations were not considered sufficient evidence for a strong claim of synergy, and these pairwise interactions were classified as additive, the null hypothesis for all pairs. Finally, two unexpected edge cases emerged in the ACVR1 knockdown. The combination of BMP4 with BMP9 generated both positive and negative IC values, though the positive IC values were larger (Figure $\mathrm{S} 2.4 \mathrm{D}$ ). However, the negative IC effect was larger in absolute terms and consistent strong antagonism observed in similar rims, such as BMP5 with BMP9. Therefore, this pair was summarized by only its largest negative IC value. Lastly, BMP2 with BMP10 appeared to be a borderline suppressive interaction. A closer examination of this pair at higher concentrations and more ligand ratios confirmed the suppression between BMP2 and BMP10, and this IC value was used to classify these ligands (Figures S2.4E,F).

\section{Interaction Coefficient provides useful classification and agrees with existing methods}

The Interaction Coefficient (IC) enabled several parts of the analysis presented here. First, as a dimensionless quantity, it allowed easy comparison between cell lines with drastically different dynamic ranges, either due to differences in sensor behavior or data being collected on different cytometers. Second, as a quantitative, rather than qualitative, metric, IC allowed "softer" classification of pairwise interactions, since small amounts of noise could possibly generate an observation of a weak, but not strong, antagonistic interaction. Third, IC reliably distinguishes distinct behavior types for a wide array of individual ligand doses, as summarized in Figure $\mathrm{S} 2.1 \mathrm{~B}$. The few difficulties of using the IC do not limit its usefulness in this study. One possible drawback to computing IC as described here is the discontinuity of the metric, introduced to avoid divergence when $f(A)=0$ cor $f(A) \approx f(B)$. However, these discontinuities preserve better our intuitive classifications of antagonism and suppression. Another challenge is that IC is computed by sampling many ligand 
ratios, computing IC for each ratio, and then summarizing the pairwise behavior with the largest magnitude IC value, whether positive or negative. However, this is a feature of all synergy metrics, which are defined with respect to the concentrations and ratio at which the pair is studied. Rather, this highlights the importance of reporting synergy alongside the concentration regime that was sampled and recognizing that all estimates of non-additivity represent lower bounds. Ultimately, IC agrees with preexisting synergy metrics, while providing improvements specific to this application. Defining a synergy metric as distinct regimes normalized to their maximum effect size as implemented by Pamela Yeh's group ${ }^{78}$ provides the basis for IC, though with a unique definition of additivity. Similarly, IC summarizes the same features described by Relative Ligand Strength and Ligand Interference Coefficient in another study of BMP pairwise interactions, ${ }^{4}$ but with two small differences. First, an $R L S$ value less than one previously classified an interaction as ratiometric (i.e. antagonistic), but our study showed that not all non-activating ligands (e.g. $R L S 0$ ) antagonized activating ligands, as captured by IC's comparison of the combination response to activation by the stronger ligand alone. Second, nonzero LIC values earn a classification of imbalance or balance, but depend on the assumption that each ligand is individually saturating, such that any increase or decrease relative to that amount is noteworthy. However, as observed in mESCs (Figure 2.4A), not all ligands appeared to saturate the pathway. The assumptions underlying IC allow its use at subsaturating ligand concentrations, as in the case of BMP9 in mESCs.

\section{Determining equivalence groups}

To classify the functional similarity of ligands, we sought a quantitative method to summarize differences between each ligand's pattern of individual and pairwise activation. To this end, we used hierarchical clustering to summarize the hierarchy of possible ligand groupings. First, we computed ligand differences as the Euclidean distance between each ligand's eleven features in a given cell line (i.e. nine IC values in pairs with the nine other ligands, one IC value in pair with itself, and one RLS value). RLS is multiplied by two for reasons described below. Next, we agglomeratively clustered ligands with complete linkage, such that the differences between two clusters is equal to the distance between their two most dissimilar members. Finally, we cut the resulting dendrogram to generate "monochromatic clusters" (cf. [62]), such that ligands in the same group had similar activation strengths and had the same interaction type (i.e. color of synergistic, additive, antagonistic, suppressive), though not necessarily interaction strength, with all other ligands. The definition of our metrics allowed us to define a distance threshold of 1 , which roughly corresponds with the desire for monochromaticity. Specifically, a distance of 1 corresponds with a difference of 1 in IC, which is the distance between the different regimes of IC behavior, or corresponds to a change of 0.5 in RLS (when RLS has been multiplied by 2), which is the difference 
between a strong and weak activator. Thus, most groups are defined by clusters at a distance of 1 , with some small adjustments to preserve monochromaticity.

\section{Global equivalence groups}

When determining global equivalence groups, each ligand has seven times as many features (i.e. IC and RLS values) than when determining equivalence in a single cell line. To maintain a similar threshold, we increased the squared distance threshold seven-fold from 1 to 7 , and cut the tree at a Euclidean distance of 7 . The five resulting equivalence groups are in fact more robust to choice of distance than other possible groupings, as they are consistent with the widest possible range of thresholds. This threshold increase allows ligands in the same global equivalence group to have small differences in pairwise interactions, meaning the global equivalence groups are not strictly "monochromatic." However, since a claim of global inequivalence is a stronger claim than inequivalence in a single cell line, this reflects our intuition that a larger pattern of differences is required to assign ligands to distinct groups and reduces the influence of a single pairwise measurement on the global classification. This is important because the three ligands whose global equivalence would be most affected by lowering the threshold, GDF5, GDF6, and GDF7, had particularly challenging to identify pairwise interactions, as a result of being non-activators. Specifically, GDFs were placed in separate equivalence groups only when they differed in their ability to produce antagonistic interactions (NMuMG cells, mESCs, ACVR1 knockdown, ACVRL1 ectopic expression) or when they differed in strength of antagonism (BMPR1B ectopic expression). However, a ligand's inability to produce an antagonistic interaction may not reflect its intrinsic properties, but instead indicate that it is present at too low a concentration. For non-activating ligands, it is difficult to determine which concentrations are sufficiently high and, in particular, whether all non-activators should be used at the same concentration. Indeed, GDF dose-responses in the single cell context where they activated, BMPR1B ectopic expression, showed significant differences in the three ligands' EC50 values (Figure S2.6E, inset). Therefore, before confidently placing GDFs in separate global equivalence groups, the lack of antagonism or suppression in some cases should be confirmed by repeating experiments with more diverse GDF concentrations.

\section{BMP Mathematical Model}

\section{Model assumptions and justification}

Dimeric BMP ligands activate the BMP pathway by complexing with two Type I and two Type II receptors. Once the complex is assembled, constitutively active Type II receptors phosphorylate 
and activate Type I receptors, which enzymatically activate SMAD1/5/8 molecules, generating a pool of pSMAD that translocates to the nucleus to promote gene expression. To focus on a minimal number of pathway components and steps, our model of BMP pathway activation considered only one-step formation of a trimeric signaling complex, containing a ligand, a Type I receptor, and a Type II receptor. In the model, each ligand binds each receptor dimer with a unique affinity and activates with a unique activity. Ligand specific-activity is a well-documented phenomenon in BMP signaling, though the mechanism is not clear. ${ }^{44}$ This simplified model neglects certain details of BMP pathway activation, which decreases model complexity without significant loss of explanatory power for our in vitro system. Specifically, one-step complex formation overlooks the stepwise assembly of BMP signaling complexes. However, summarizing a two-step binding process with a single phenomenological parameter does not limit the possible pairwise interactions allowed by the pathway architecture. ${ }^{68}$ The reduced model also neglects the pentameric nature of the full BMP signaling complex, where one BMP dimer binds two Type I and two Type II receptors. This model also does not include the other secreted and membrane-bound molecules that can influence signaling complex formation, such as secreted ligand antagonists (e.g. Twsg1, Noggin, Chordin), co-receptors (e.g. RGMA, ENG), pseudo-receptors (e.g. BAMBI), or other inhibitors (e.g. Fst). However, siRNA targeting the most highly-expressed of these molecules in NMuMG, including Twsg1, Fst, and Rgmb, had no effect on pairwise interactions in NMuMG..$^{4}$

\section{Model equations and solution}

To model BMP pathway activation, we used mass-action kinetics to describe the one-step formation of tripartite signaling complexes $\left(T_{i j k}\right)$, where one ligand $\left(L_{i}\right)$ binds one Type I receptor $\left(A_{j}\right)$ and one Type II receptor $\left(B_{k}\right)$ with a complex-specific affinity $\left(K_{i j k}\right)$. Each complex also has a distinct activity $\left(\epsilon_{i j k}\right)$ with which it phosphorylates downstream SMAD proteins, and each complex contributes to the overall measured output $(S)$. We assume that binding kinetics reach steady-state more rapidly than complexes activate the pathway. We also assume that ligand concentration, provided by a large reservoir of cell media in experiments, is effectively constant. As shown in [68], this results in the final equations: 


$$
\begin{aligned}
S & =\sum_{i j k} \epsilon_{i j k} T_{i j k} \\
T_{i j k} & =K_{i j k} L_{i}^{0} A_{j} B_{k} \\
A_{j} & =A_{j}^{0}-\sum_{i k} T_{i j k} \\
B_{k} & =B_{k}^{0}-\sum_{i j} T_{i j k}
\end{aligned}
$$

where $L_{i}^{0}, A_{j}^{0}$, and $B_{k}^{0}$ correspond to the starting concentrations of these free species. Thus, when the receptor and ligand environment are specified (i.e. $L_{i}^{0}, A_{j}^{0}$, and $B_{k}^{0}$ are known), pathway activation can be solved for, as a function of independent variables are $K_{i j k}$ and $\epsilon_{i j k}$. The implicit dependence of $S$ on the model parameters can be solved by constrained optimization, implemented by the Python package promisys. $\underline{68}$

\section{Parameter fitting}

To maximally constrain the fit parameters, we selected datasets that could be fit by the fewest parameters, which scale with the number of ligand and receptor components. To this end, the model was fit to five ligands signaling in cell lines that could be modeled with only five of the seven BMP receptors. Specifically, we focused on BMP4, BMP7, BMP9, BMP10, and GDF5, representing each one of the global equivalence groups and neglecting possible differences between GDFs. We further selected the NMuMG and receptor knockdown datasets, which express the fluorescent BMP reporter from the same genomic locus and express at most five BMP receptors. Receptor expression in these four cell lines was also measured by the same RT-qPCR protocol. As indicated in the model, our measurements of BMP signaling can thus be modeled by $\mathrm{S}$ and its dependence on $L_{i}^{0}$, $A_{j}^{0}, B_{k}^{0}$, the known ligand and receptor environments, and $K_{i j k}$ and $\epsilon_{i j k}$, the unknown affinities and activities that are fit. However, the data carry various units that also appear in the fit parameters. $S$ is measured in units of background-subtracted median YFP, $L_{i}^{0}$ in units of $\mathrm{ng} / \mathrm{mL}$, and $A_{j}^{0}$ and $B_{k}^{0}$ in relative expression units from RT-qPCR abundance normalized to a housekeeping gene. The fit parameters units depend on these values in the following way: 


$$
\begin{gathered}
T_{i j k}[=] A_{j} \\
K_{i j k}[=] \frac{1}{L_{i} A_{j}} \\
\epsilon_{i j k}[=] \frac{S}{A_{j}}
\end{gathered}
$$

where [=] indicates two quantities having equivalent units. The implicit units in the fit parameters influenced the parameter fitting in the following two ways. First, any global transform of $S, L_{i}^{0}$, $A_{j}^{0}$, or $B_{k}^{0}$ affects absolute parameter values to reflect the change in units, but does not affect the overall fit. Therefore, for convenience, all values of $S, A_{j}^{0}$, or $B_{k}^{0}$ were normalized to a maximum of 1, dividing by the maximum YFP or relative expression value, respectively, across all four cell lines. Second, the lack of meaningful units for the $K_{i j k}$ and $\epsilon_{i j k}$ parameters made it difficult to define biologically-reasonable constraints on the absolute values of these parameters. In principle, the values of $K_{i j k}$ and $\epsilon_{i j k}$ may take any nonnegative value, but they cannot physically vary over an arbitrary number of orders of magnitude. Therefore, $K_{i j k}$ and $\epsilon_{i j k}$ were bounded within six orders of magnitude. To find reasonable upper and lower bounds, a subset of the data was fit with strictly positive $K_{i j k}$ and $\epsilon_{i j k}$ values, which used a method similar to the one described below but did not constrain parameters and produced dozens of equivalently good solutions. These sets of solutions showed most best fit parameter values for $K_{i j k}$ and $\epsilon_{i j k}$ ranged between $10^{-4}$ and $10^{2}$ when $S$ and receptor expression were normalized between 0 and 1 . A final consideration in model fitting was the nature of RT-qPCR measurements, where different gene targets are amplified with different probe sets with different efficiencies. These values introduce significantly larger error than $L_{i}^{0}$, which is directly controlled, and $\mathrm{S}$, which is a median across hundreds of cells. At the high $C_{t}$ values of these relatively low abundance receptors, estimates of the same level of expression could vary as much as 10-fold, assuming a 10\% difference of efficiency amplified over 25 cycles. As relative receptor levels are crucial in the model, a rescaling parameter, bounded by a 10-fold increase or decrease, was added for each receptor, to reflect the uncertainty of the RT-qPCR estimate of gene expression. Thus, the values passed to the model are instead:

$$
\begin{aligned}
& A_{j}^{0}=\rho_{j} A_{j}^{0, q P C R} \\
& B_{k}^{0}=\rho_{k} B_{k}^{0, q P C R}
\end{aligned}
$$

where $A_{j}^{0, q P C R}$ and $B_{k}^{0, q P C R}$ are total receptor expression measured by RT-qPCR and $\rho_{j}$ and $\rho_{k}$ are their respective rescaling parameters. To fit $K_{i j k}$ and $\epsilon_{i j k}$, we used SciPy's least squares 
function to minimize the error between observed and model-predicted $S$, when $L_{i}^{0}, A_{j}^{0}$, and $B_{k}^{0}$ were specified and $K_{i j k}, \epsilon_{i j k}, \rho_{j}$, and $\rho_{k}$ freely varied over the ranges described above. Each parameter fitting run was initiated with a distinct, randomly-chosen initial condition and resulted in an equal number of distinct solutions, each with some total error. Filtering out solutions whose error changed the type, rather than strength, of pairwise interactions between ligands, we identified a set of 22 solutions that most closely matched the data's qualitative behavior (Figure $\mathrm{S} 2.8 \mathrm{~B}$ ). These fit parameters are stored in a pickle file parameter_fitting_filtered_fits.pkl, deposited at https://data.caltech.edu/records/1693. 


\subsection{Key Resources and Tables}

\section{Table 2.1: Key Resources}

\begin{tabular}{|c|c|c|}
\hline Reagent or Resource & Source & Identifier \\
\hline \multicolumn{3}{|c|}{ Chemicals, Peptides, and Recombinant Proteins } \\
\hline Fetal Bovine Serum & VWR & $311 \mathrm{~K} 18$ \\
\hline Fetal Bovine Serum (ES qualified) & ThermoFisher & 16141 \\
\hline Leukemia Inhibiting Factor & ThermoFisher & Cat\#ESG1107 \\
\hline RNAiMAX & ThermoFisher & Cat\#13778075 \\
\hline Lipofectamine LTX & ThermoFisher & Cat\#15338100 \\
\hline Trypsin $(0.25 \%)$ & ThermoFisher & Cat\#25200056 \\
\hline Accutase & ThermoFisher & Cat\#A1110501 \\
\hline BMP ligands & R\&D Biosystems & See Table 2.2 \\
\hline \multicolumn{3}{|c|}{ Critical Commercial Assay } \\
\hline RNAeasy mini kit & QIAGEN & Cat\#74104 \\
\hline iScript cDNA synthesis kit & BioRad & Cat\#1708890 \\
\hline IQ SYBR Green Supermix & BioRad & Cat\#1708882 \\
\hline $\begin{array}{l}\text { SsoAdvanced Universal probes Su- } \\
\text { permix }\end{array}$ & BioRad & Cat\#1725281 \\
\hline \multicolumn{3}{|c|}{ Deposited Data } \\
\hline All flow cytometry data & This paper & https://data.caltech.edu/ \\
\hline All How cytometry data & IIIs paper & $\begin{array}{l}\text { records/1693 } \\
\text { https://data.caltech.edu/ }\end{array}$ \\
\hline All RT-qPCR data & This paper & $\begin{array}{l}\text { nttps://aata.caltecn.eau/ } \\
\text { records/1693 }\end{array}$ \\
\hline \multicolumn{3}{|c|}{ Experimental Models: Cell Lines } \\
\hline NMuMG & ATCC & CRL-1636 \\
\hline NMuMG sensor line & This paper & N/A \\
\hline mESC sensor line & $\begin{array}{l}\text { Antebi, et al. } \\
2017\end{array}$ & N/A \\
\hline $\begin{array}{l}\text { NMuMG sensor with ectopic } \\
\text { BMPR1B }\end{array}$ & This paper & N/A \\
\hline $\begin{array}{l}\text { NMuMG sensor with ectopic } \\
\text { BMPR1B }\end{array}$ & This paper & N/A \\
\hline NMuMG sensor with BMPR2 shRNA & This paper & N/A \\
\hline NMuMG sensor with ACVR1 shRNA & This paper & N/A \\
\hline $\begin{array}{l}\text { NMuMG sensor with BMPR1A } \\
\text { shRNA }\end{array}$ & This paper & N/A \\
\hline \multicolumn{3}{|c|}{ Oligonucleotides } \\
\hline siRNA targeting BMP receptors & $\begin{array}{l}\text { Antebi, et al. } \\
2017\end{array}$ & See Table 2.4 \\
\hline $\begin{array}{l}\mathrm{qPCR} \text { primers and probes for } \mathrm{BMP} \\
\text { receptors }\end{array}$ & $\begin{array}{l}\text { Antebi, et al. } \\
2017\end{array}$ & See Table 2.5 \\
\hline \multicolumn{3}{|c|}{ Recombinant DNA } \\
\hline pHK004_PB_MLP_BRE_H2B-C_puro & This paper & N/A \\
\hline \multicolumn{3}{|c|}{ Software and Algorithms } \\
\hline MATLAB & MathWorks & N/A \\
\hline Python & $\begin{array}{l}\text { Python Software } \\
\text { Foundation }\end{array}$ & N/A \\
\hline $\begin{array}{l}\text { Easyflow (MATLAB-based flow cy- } \\
\text { tometry analysis software) }\end{array}$ & Antebi lab & $\begin{array}{l}\text { https://github.com/AntebiLab/ } \\
\text { easyflow }\end{array}$ \\
\hline $\begin{array}{l}\text { Promisys (Python code for simulating } \\
\text { ligand-receptor interactions) }\end{array}$ & Su et al. & $\begin{array}{l}\text { https://data.caltech.edu/ } \\
\text { records/1692 }\end{array}$ \\
\hline \multicolumn{3}{|c|}{ Other } \\
\hline $\begin{array}{l}\text { SMARTvector } \quad \text { BMPR2-targeting } \\
\text { shRNA viral particles (mEF1a-RFP) }\end{array}$ & Dharmacon & V3SM7598-10EG12168 (see Table 2.3 \\
\hline $\begin{array}{l}\text { SMARTvector BMPR1A-targeting } \\
\text { shRNA viral particles (mEF1a-RFP) }\end{array}$ & Dharmacon & V3SM7598-10EG12166 (see Table 2.3 \\
\hline $\begin{array}{l}\text { SMARTvector ACVR1-targeting } \\
\text { shRNA viral particles (mEF1a-RFP) }\end{array}$ & Dharmacon & V3SM7598-10EG11477 (see Table 2.3 \\
\hline $\begin{array}{l}\text { SMARTvector GAPDH-targeting } \\
\text { shRNA viral particles (mEF1a-RFP) }\end{array}$ & Dharmacon & S10-002000-01 \\
\hline $\begin{array}{l}\text { SMARTvector Non-targeting shRNA } \\
\text { viral particles (mEF1a-RFP) }\end{array}$ & Dharmacon & S10-005000-01 \\
\hline
\end{tabular}


Table 2.2: Recombinant BMP Ligands and Concentrations

\begin{tabular}{|c|c|c|c|c|c|c|c|c|c|c|c|}
\hline & Ligand & BMP2 & BMP4 & BMP5 & BMP6 & BMP7 & BMP9 & BMP10 & GDF5 & GDF6 & GDF7 \\
\hline & Cat. Number (R\&D) & $\begin{array}{l}355- \\
\text { BM- } \\
050\end{array}$ & $\begin{array}{l}5020- \\
\text { BP }\end{array}$ & $\begin{array}{l}\text { 6176- } \\
\text { BM }\end{array}$ & $\begin{array}{l}6325- \\
\text { BM- } \\
020\end{array}$ & $\begin{array}{l}5666- \\
\text { BP }\end{array}$ & $\begin{array}{l}5566- \\
\text { BP }\end{array}$ & $\begin{array}{l}6038- \\
\text { BP }\end{array}$ & $\begin{array}{l}853- \\
\text { G5 }\end{array}$ & $\begin{array}{l}855- \\
\text { G6- } \\
050\end{array}$ & $\begin{array}{l}\text { 779- } \\
\text { G7 }\end{array}$ \\
\hline \multirow{2}{*}{ NMuMG } & max. conc. $(\mathrm{ng} / \mathrm{mL})$ & 759.2 & 57.2 & 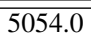 & 1837.3 & 3678.9 & 106.4 & 1298.3 & 912.2 & $\overline{9912.2}$ & 912.2 \\
\hline & Fold dilution & 12.0 & 55.9 & 3.46 & 4.00 & 3.12 & 4.15 & 9.86 & 15.5 & 15.5 & 15.5 \\
\hline \multirow{2}{*}{ mESC } & max. conc. $(\mathrm{ng} / \mathrm{mL})$ & 1000 & 1000 & 2000 & 2000 & 2000 & 1000 & 1000 & 1000 & 1000 & 1000 \\
\hline & Fold dilution & 10 & 10 & 2 & 2 & 3 & 3.1 & 3.7 & 3.7 & 3.7 & 3.7 \\
\hline \multirow{2}{*}{ Ectopic BMPR1B } & max. conc. $(\mathrm{ng} / \mathrm{mL})$ & 760 & 60 & 2500 & 1000 & 2000 & 100 & 100 & 900 & 1000 & 50 \\
\hline & Fold dilution & 12 & 55 & 15 & 7 & 10 & 4 & 5 & 15 & 8 & 5 \\
\hline \multirow{2}{*}{ Ectopic ACVRL1 } & max. conc. $(\mathrm{ng} / \mathrm{mL})$ & 500 & 500 & 2000 & 2000 & 1000 & 5 & 30 & 1000 & 1000 & 1000 \\
\hline & Fold dilution & 10 & 10 & 3 & 3 & 3 & 5 & 5 & 4 & 4 & 4 \\
\hline \multirow{2}{*}{ BMPR2 knockdown } & max. conc. $(\mathrm{ng} / \mathrm{mL})$ & 1000 & 1000 & 2000 & 2000 & 1000 & 1000 & 1000 & 1000 & 1000 & 1000 \\
\hline & Fold dilution & 4 & 6 & 2 & 2 & 2 & 3 & 3 & 3 & 3 & 3 \\
\hline \multirow{2}{*}{ ACVR1 knockdown } & max. conc. $(\mathrm{ng} / \mathrm{mL})$ & 500 & 100 & 3000 & 3000 & 3000 & 3000 & 100 & 3000 & 3000 & 3000 \\
\hline & Fold dilution & 8 & 10 & 2 & 2.5 & 2 & 5 & 5 & 3 & 3 & 3 \\
\hline \multirow{2}{*}{ BMPR1A knockdown } & max. conc. $(\mathrm{ng} / \mathrm{mL})$ & 1000 & 1000 & 2000 & 2000 & 1000 & 1000 & 2000 & 1000 & 1000 & 1000 \\
\hline & Fold dilution & 1.7 & 2.7 & 1.9 & 2.9 & 2.1 & 10.9 & 3.4 & 2.7 & 2.7 & 2.7 \\
\hline
\end{tabular}

Table 2.3: shRNA Sequences

\begin{tabular}{|c|c|c|c|c|}
\hline Target & Catalog \# & Source Clone ID & Vector & Gene target sequence \\
\hline \hline BMPR2 & V3SM7592-231979356 & V3SVMM10_11917013 & pSMART_mEF1a/TurboRFP & ACAAGCAAATACTCCATGC \\
BMPR2 & V3SM7592-234523888 & V3SVMM10_14461544 & pSMART_mEF1a/TurboRFP & TATCGACCCCGTCCAATCA \\
BMPR2 & V3SM7592-235657177 & V3SVMM10_15594830 & pSMART_mEF1a/TurboRFP & AGACCATAACACGTGCTCC \\
\hline \hline BMPR1A & V3SM7592-232280382 & V3SVMM10_12248039 & pSMART_mEF1a/TurboRFP & GTGTGAAACGCTTGCGGCC \\
BMPR1A & V3SM7592-233338234 & V3SVMM10_13275887 & pSMART_mEF1a/TurboRFP & GGAGTGGATCTGGATTGCC \\
BMPR1A & V3SM7592-235771879 & V3SVMM10_15709538 & pSMART_mEF1a/TurboRFP & AAATGGAAGTTGCTGTATT \\
\hline \hline ACVR1 & V3SM7592-231384102 & V3SVMM10_11321759 & pSMART_mEF1a/TurboRFP & ATAAGAGGGTCGATATTTG \\
ACVR1 & V3SM7592-232622298 & V3SVMM10_12559952 & pSMART_mEF1a/TurboRFP & AATAGGAATTCAATCTGGC \\
ACVR1 & V3SM7592-236015386 & V3SVMM10_15953045 & pSMART_mEF1a/TurboRFP & GATCACTCGTGTACATCAG \\
\hline
\end{tabular}

Table 2.4: siRNA Sequences

\begin{tabular}{|c|c|c|}
\hline RNA & Source & ID \# \\
\hline \hline BMPR1A siRNA & Lifetech & S201096 \\
BMPR1A siRNA & Lifetech & S201097 \\
\hline \hline BMPR2 siRNA & Lifetech & S63047 \\
BMPR2 siRNA & Lifetech & S63048 \\
\hline \hline ACVR1 siRNA & Lifetech & S61924 \\
ACVR1 siRNA & Lifetech & S61925 \\
\hline
\end{tabular}




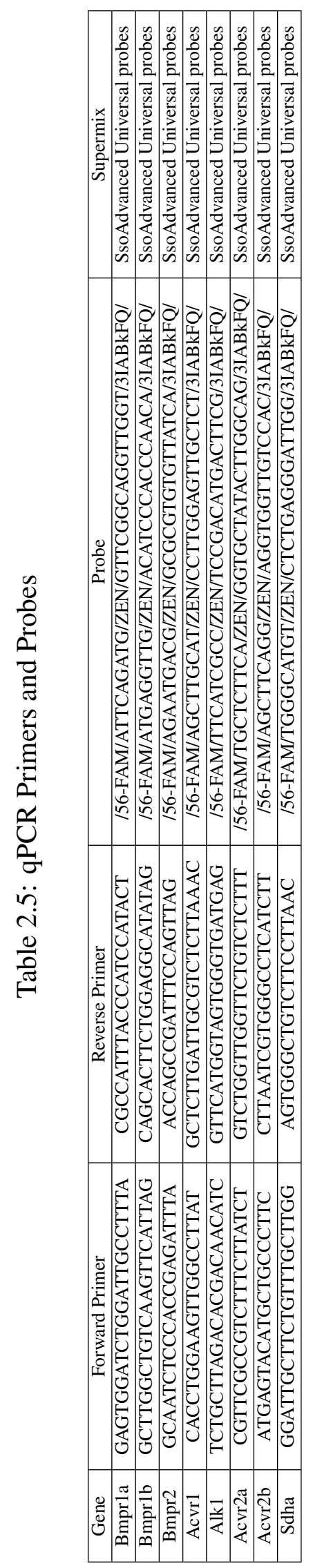




\section{References}

[1] Shailesh Agarwal et al. "Strategic Targeting of Multiple BMP Receptors Prevents TraumaInduced Heterotopic Ossification". en. In: Mol. Ther. 25.8 (Aug. 2017), pp. 1974-1987. ISSN: 1525-0016, 1525-0024. DOI: $10.1016 / \mathrm{j}$. ymthe.2017.01.008.

[2] Anshula Alok et al. "Wnt proteins synergize to activate $\beta$-catenin signaling". en. In: J. Cell Sci. 130.9 (May 2017), pp. 1532-1544. ISSN: 0021-9533, 1477-9137. DoI: $10.1242 /$ jcs. 198093 .

[3] Madeline G Andrews et al. "BMPs direct sensory interneuron identity in the developing spinal cord using signal-specific not morphogenic activities". en. In: Elife 6 (Sept. 2017). ISSN: 2050-084X. DOI: $10.7554 /$ eLife.30647.

[4] Yaron E Antebi et al. "Combinatorial Signal Perception in the BMP Pathway". en. In: Cell 170.6 (Sept. 2017), 1184-1196.e24. ISSN: 0092-8674, 1097-4172. DOI: $10.1016 / j$.cell. 2017.08.015.

[5] Senem Aykul and Erik Martinez-Hackert. "Transforming Growth Factor- $\beta$ Family Ligands Can Function as Antagonists by Competing for Type II Receptor Binding". en. In: J. Biol. Chem. 291.20 (May 2016), pp. 10792-10804. ISSN: 0021-9258, 1083-351X. DoI: 10.1074/ jbc.M115.713487.

[6] Senem Aykul et al. "Activin A forms a non-signaling complex with ACVR1 and type II Activin/BMP receptors via its finger 2 tip loop". en. In: Elife 9 (June 2020). ISSN: 2050084X. DoI: $10.7554 /$ eLife. 54582 .

[7] Amitabha Bandyopadhyay et al. "Genetic analysis of the roles of BMP2, BMP4, and BMP7 in limb patterning and skeletogenesis". en. In: PLoS Genet. 2.12 (Dec. 2006), e216. ISSN: 1553-7390, 1553-7404. DoI: $10.1371 /$ journal.pgen.0020216.

[8] Brian Bierie and Harold L Moses. "Transforming growth factor beta (TGF-beta) and inflammation in cancer". en. In: Cytokine Growth Factor Rev. 21.1 (Feb. 2010), pp. 49-59. ISSN: 1359-6101, 1879-0305. DoI: $10.1016 /$ j . cytogfr.2009.11.008

[9] Chester I Bliss. “The toxicity of poisons applied jointly 1". In: Ann. Appl. Biol. 26.3 (1939), pp. 585-615. ISSN: 0003-4746.

[10] Ira L Blitz and Ken W Y Cho. "Finding partners: how BMPs select their targets". en. In: Dev. Dyn. 238.6 (June 2009), pp. 1321-1331. ISSN: 1058-8388. Dor: 10.1002/dvdy. 21984.

[11] Tobias Bollenbach et al. "Nonoptimal microbial response to antibiotics underlies suppressive drug interactions”. en. In: Cell 139.4 (Nov. 2009), pp. 707-718. ISSN: 0092-8674, 1097-4172. DOI: $10.1016 / \mathrm{j} . \mathrm{cell} .2009 .10 .025$.

[12] Samantha J Butler and Jane Dodd. "A role for BMP heterodimers in roof plate-mediated repulsion of commissural axons". en. In: Neuron 38.3 (May 2003), pp. 389-401. ISSN: 0896-6273. DOI: 10.1016/s0896-6273(03)00254-x. 
[13] Eugene J Carragee, Eric L Hurwitz, and Bradley K Weiner. "A critical review of recombinant human bone morphogenetic protein-2 trials in spinal surgery: emerging safety concerns and lessons learned". en. In: Spine J. 11.6 (June 2011), pp. 471-491. issN: 1529-9430, 1878-1632. DOI: $10.1016 / \mathrm{j}$.spinee.2011.04.023.

[14] Xiao-Qing Chai et al. Role of activin receptor-like kinase 1 in vascular development and cerebrovascular diseases. 2020. DOI: 10.4103/1673-5374.280305.

[15] H Chen et al. "Context-dependent signaling defines roles of BMP9 and BMP10 in embryonic and postnatal development". In: Proceedings of the National Academy of Sciences 110.29 (2013), pp. 11887-11892. DoI: 10.1073/pnas.1306074110.

[16] Marco Cicciù et al. "Recombinant human bone morphogenetic protein-2 promote and stabilize hard and soft tissue healing for large mandibular new bone reconstruction defects". en. In: J. Craniofac. Surg. 25.3 (May 2014), pp. 860-862. ISSN: 1049-2275, 1536-3732. DoI: $10.1097 /$ SCS. 0000000000000830 .

[17] Michael Costanzo et al. "A global genetic interaction network maps a wiring diagram of cellular function”. en. In: Science 353.6306 (Sept. 2016). ISSN: 0036-8075, 1095-9203. DoI: 10.1126/science.aaf1420.

[18] April M Craft et al. "Specification of chondrocytes and cartilage tissues from embryonic stem cells". en. In: Development 140.12 (June 2013), pp. 2597-2610. ISSN: 0950-1991, 1477-9129. DOI: $10.1242 / \mathrm{dev} .087890$.

[19] Sara I Cunha and Kristian Pietras. "ALK1 as an emerging target for antiangiogenic therapy of cancer". en. In: Blood 117.26 (June 2011), pp. 6999-7006. ISSN: 0006-4971, 1528-0020. DOI: $10.1182 /$ blood-2011-01-330142.

[20] Shahab M Danesh et al. "BMP and BMP receptor expression during murine organogenesis". en. In: Gene Expr. Patterns 9.5 (June 2009), pp. 255-265. IssN: 1567-133X. DoI: 10.1016/ j.gep.2009.04.002.

[21] Charles J David and Joan Massagué. "Contextual determinants of TGF $\beta$ action in development, immunity and cancer”. en. In: Nat. Rev. Mol. Cell Biol. 19.7 (July 2018), pp. 419-435. ISSN: 1471-0072, 1471-0080. DOI: 10.1038/s41580-018-0007-0.

[22] L David et al. "Identification of BMP9 and BMP10 as functional activators of the orphan activin receptor-like kinase 1 (ALK1) in endothelial cells". In: Blood (2007). ISSN: 00064971.

[23] Christopher Ede et al. "Quantitative Analyses of Core Promoters Enable Precise Engineering of Regulated Gene Expression in Mammalian Cells". en. In: ACS Synth. Biol. 5.5 (May 2016), pp. 395-404. ISSN: 2161-5063. DOI: 10.1021/acssynbio.5b00266.

[24] Julie E Gleason, Elizabeth A Szyleyko, and David M Eisenmann. Multiple redundant Wnt signaling components function in two processes during C. elegans vulval development. 2006. DOI: $10.1016 / \mathrm{j}$.ydbio.2006.06.050.

[25] R E Godin, E J Robertson, and A T Dudley. "Role of BMP family members during kidney development”. en. In: Int. J. Dev. Biol. 43.5 (1999), pp. 405-411. ISSN: 0214-6282. 
[26] Valerie Gouon-Evans et al. "BMP-4 is required for hepatic specification of mouse embryonic stem cell-derived definitive endoderm". en. In: Nat. Biotechnol. 24.11 (Nov. 2006), pp. 14021411. ISSN: 1087-0156. DOI: $10.1038 /$ nbt 1258 .

[27] I Greenwald and G M Rubin. "Making a difference: the role of cell-cell interactions in establishing separate identities for equivalent cells". en. In: Cell 68.2 (Jan. 1992), pp. 271281. ISSN: 0092-8674. DOI: 10.1016/0092-8674(92) 90470-w.

[28] Lea M Gunnell et al. "TAK1 regulates cartilage and joint development via the MAPK and BMP signaling pathways". en. In: J. Bone Miner. Res. 25.8 (Aug. 2010), pp. 1784-1797. ISSN: 0884-0431, 1523-4681. DOI: $10.1002 / \mathrm{jbmr} .79$.

[29] Chul Han et al. "SMAD1 deficiency in either endothelial or smooth muscle cells can predispose mice to pulmonary hypertension". en. In: Hypertension 61.5 (May 2013), pp. 10441052. ISSN: 0194-911X, 1524-4563. DOI: 10.1161/HYPERTENSIONAHA.111.199158.

[30] Kai Heinecke et al. "Receptor oligomerization and beyond: a case study in bone morphogenetic proteins". en. In: BMC Biol. 7 (Sept. 2009), p. 59. IsSN: 1741-7007. Dor: 10.1186/ 1741-7007-7-59.

[31] Christian Hiepen et al. "BMPR2 acts as a gatekeeper to protect endothelial cells from increased TGF $\beta$ responses and altered cell mechanics". en. In: PLoS Biol. 17.12 (Dec. 2019), e3000557. ISSN: 1544-9173, 1545-7885. Dor: 10.1371/journal.pbio.3000557.

[32] Andrew P Hinck, Thomas D Mueller, and Timothy A Springer. "Structural Biology and Evolution of the TGF- $\beta$ Family". en. In: Cold Spring Harb. Perspect. Biol. 8.12 (Dec. 2016). ISSN: 1943-0264. DOI: 10.1101/cshperspect.a022103

[33] Jie Huang et al. "Negative and positive auto-regulation of BMP expression in early eye development". en. In: Dev. Biol. 407.2 (Nov. 2015), pp. 256-264. ISSN: 0012-1606, 1095564X. DOI: $10.1016 / \mathrm{j}$. ydbio.2015.09.009.

[34] Michael J Isaacs et al. "Bone morphogenetic protein-2 and -6 heterodimer illustrates the nature of ligand-receptor assembly". en. In: Mol. Endocrinol. 24.7 (July 2010), pp. 14691477. ISSN: 0888-8809, 1944-9917. Dor: 10.1210/me.2009-0496.

[35] Snehalata Kadam, Srimoyee Ghosh, and Angelike Stathopoulos. "Synchronous and symmetric migration of Drosophila caudal visceral mesoderm cells requires dual input by two FGF ligands". en. In: Development 139.4 (Feb. 2012), pp. 699-708. ISSN: 0950-1991, 1477-9129. DOI: $10.1242 / \mathrm{dev} .068791$.

[36] Snehalata Kadam et al. "FGF ligands in Drosophila have distinct activities required to support cell migration and differentiation". en. In: Development 136.5 (Mar. 2009), pp. 739-747. ISSN: 0950-1991. DOI: $10.1242 / \mathrm{dev} .027904$.

[37] Päivi Kettunen and Irma Thesleff. "Expression and function of FGFs-4,-8, and-9 suggest functional redundancy and repetitive use as epithelial signals during tooth morphogenesis". In: Dev. Dyn. 211.3 (1998), pp. 256-268. ISSN: 1058-8388.

[38] Uwe Klammert et al. "GDF-5 can act as a context-dependent BMP-2 antagonist". en. In: BMC Biol. 13 (Sept. 2015), p. 77. ISSN: 1741-7007. DoI: 10.1186/s12915-015-0183-8. 
[39] Olexander Korchynskyi and Peter ten Dijke. "Identification and functional characterization of distinct critically important bone morphogenetic protein-specific response elements in the Id1 promoter". en. In: J. Biol. Chem. 277.7 (Feb. 2002), pp. 4883-4891. ISSN: 0021-9258. DOI: $10.1074 / \mathrm{jbc} . \mathrm{M} 111023200$.

[40] Alexander Lachmann et al. "Massive mining of publicly available RNA-seq data from human and mouse". en. In: Nat. Commun. 9.1 (Apr. 2018), p. 1366. ISSN: 2041-1723. DoI: 10.1038/s41467-018-03751-6.

[41] S Loewe. "Die quantitativen Probleme der Pharmakologie". In: Ergeb. Physiol. 27.1 (Dec. 1928), pp. 47-187. ISSN: 0080-2042. DOI: 10.1007/BF02322290.

[42] Jonathan W Lowery et al. "Loss of BMPR2 leads to high bone mass due to increased osteoblast activity". en. In: J. Cell Sci. 128.7 (Apr. 2015), pp. 1308-1315. ISSN: 0021-9533, 1477-9137. DOI: $10.1242 /$ jcs. 156737

[43] Christian T Meyer et al. "Quantifying Drug Combination Synergy along Potency and Efficacy Axes". en. In: Cell Syst 8.2 (Feb. 2019), 97-108.e16. ISSN: 2405-4712. DoI: $10.1016 /$ j . cels.2019.01.003

[44] Joachim Nickel and Thomas D Mueller. "Specification of BMP Signaling". en. In: Cells 8.12 (Dec. 2019). ISSN: 2073-4409. DOI: 10.3390/cells8121579.

[45] H Nishitoh et al. "Identification of type I and type II serine/threonine kinase receptors for growth/differentiation factor-5". en. In: J. Biol. Chem. 271.35 (Aug. 1996), pp. 21345-21352. ISSN: 0021-9258. DOI: $10.1074 / \mathrm{jbc} .271 .35 .21345$.

[46] Roel Nusse. "Wnt signaling in disease and in development". en. In: Cell Res. 15.1 (Jan. 2005), pp. 28-32. ISSN: 1001-0602. DoI: 10.1038/sj.cr.7290260.

[47] Oddrun Elise Olsen et al. "BMPR2 inhibits activin and BMP signaling via wild-type ALK2". en. In: J. Cell Sci. 131.11 (June 2018). ISSN: 0021-9533, 1477-9137. DOI: $10.1242 /$ jcs. 213512.

[48] David M Ornitz and Nobuyuki Itoh. The Fibroblast Growth Factor signaling pathway. 2015. DOI: $10.1002 / \mathrm{wdev} .176$.

[49] Mar Orriols, Maria Catalina Gomez-Puerto, and Peter Ten Dijke. "BMP type II receptor as a therapeutic target in pulmonary arterial hypertension". en. In: Cell. Mol. Life Sci. 74.16 (Aug. 2017), pp. 2979-2995. ISSN: 1420-682X, 1420-9071. Dor: 10.1007/s00018-017-2510-4.

[50] Leif Oxburgh et al. "BMP4 substitutes for loss of BMP7 during kidney development". en. In: Dev. Biol. 286.2 (Oct. 2005), pp. 637-646. ISSN: 0012-1606. Dor: $10.1016 / j$. ydbio. 2005.08 .024

[51] Mohammadreza Pakyari et al. "Critical Role of Transforming Growth Factor Beta in Different Phases of Wound Healing". en. In: Adv. Wound Care 2.5 (June 2013), pp. 215-224. issN: 1076-2191, 2162-1918. DOI: $10.1089 /$ wound.2012.0406.

[52] D M Panchision et al. "Sequential actions of BMP receptors control neural precursor cell production and fate". en. In: Genes Dev. 15.16 (Aug. 2001), pp. 2094-2110. ISSN: 0890-9369. DOI: $10.1101 / \mathrm{gad} .894701$. 
[53] E Piek et al. "TGF-(beta) type I receptor/ALK-5 and Smad proteins mediate epithelial to mesenchymal transdifferentiation in NMuMG breast epithelial cells". en. In: J. Cell Sci. 112 ( Pt 24) (Dec. 1999), pp. 4557-4568. ISSN: 0021-9533.

[54] T D Piscione et al. "BMP-2 and OP-1 exert direct and opposite effects on renal branching morphogenesis”. en. In: Am. J. Physiol. 273.6 (Dec. 1997), F961-75. ISSN: 0002-9513. DOI: 10.1152/ajprenal.1997.273.6.F961.

[55] P Razavi, N Lopez, and K M Lyons. "The TGF $\beta$ type I receptor TGF $\beta$ RI functions as an inhibitor of BMP signaling in cartilage”. In: Proceedings of the (2019).

[56] D Russ and R Kishony. "Additivity of inhibitory effects in multidrug combinations". en. In: Nat Microbiol 3.12 (Dec. 2018), pp. 1339-1345. ISSN: 2058-5276. DOI: 10.1038/s41564018-0252-1.

[57] Valerie S Salazar, Laura W Gamer, and Vicki Rosen. "BMP signalling in skeletal development, disease and repair”. en. In: Nat. Rev. Endocrinol. 12.4 (Apr. 2016), pp. 203-221. ISSN: 1759-5029, 1759-5037. Dor: $10.1038 /$ nrendo.2016.12.

[58] Stefan Saremba et al. "Type I receptor binding of bone morphogenetic protein 6 is dependent on N-glycosylation of the ligand: Type I receptor specificity of BMP-6". en. In: FEBS J. 275.1 (Jan. 2008), pp. 172-183. ISSN: 1742-464X, 1742-4658. DOI: $10.1111 / \mathrm{j} .1742-$ $4658.2007 .06187 . \mathrm{x}$

[59] Monika Saxena, Nitin Agnihotri, and Jonaki Sen. "Perturbation of canonical and noncanonical BMP signaling affects migration, polarity and dendritogenesis of mouse cortical neurons". en. In: Development 145.1 (Jan. 2018). ISSN: 0950-1991, 1477-9129. DOI: 10. 1242 /dev. 147157

[60] Benno Schwikowski, Peter Uetz, and Stanley Fields. "A network of protein-protein interactions in yeast”. en. In: Nat. Biotechnol. 18.12 (Dec. 2000), pp. 1257-1261. ISSN: 1087-0156. DOI: $10.1038 / 82360$.

[61] Howard J Seeherman et al. "A BMP/activin A chimera is superior to native BMPs and induces bone repair in nonhuman primates when delivered in a composite matrix". en. In: Sci. Transl. Med. 11.489 (Apr. 2019). ISSN: 1946-6234, 1946-6242. DOI: 10. $1126 /$ scitranslmed.aar4953.

[62] Daniel Segrè et al. "Modular epistasis in yeast metabolism". en. In: Nat. Genet. 37.1 (Jan. 2005), pp. 77-83. ISSN: 1061-4036. DOI: $10.1038 / \mathrm{ng} 1489$

[63] Tsugio Seki, Jihye Yun, and S Paul Oh. "Arterial endothelium-specific activin receptor-like kinase 1 expression suggests its role in arterialization and vascular remodeling". en. In: Circ. Res. 93.7 (Oct. 2003), pp. 682-689. ISSN: 0009-7330, 1524-4571. DOI: 10.1161/01. RES. $0000095246.40391 .3 \mathrm{~B}$

[64] Shunichi Shimasaki et al. "The bone morphogenetic protein system in mammalian reproduction". en. In: Endocr. Rev. 25.1 (Feb. 2004), pp. 72-101. ISSN: 0163-769X. DOI: $10.1210 /$ er . 2003-0007 
[65] Eileen M Shore et al. "A recurrent mutation in the BMP type I receptor ACVR1 causes inherited and sporadic fibrodysplasia ossificans progressiva". en. In: Nat. Genet. 38.5 (May 2006), pp. 525-527. ISSN: 1061-4036. DOI: 10.1038/ng1783.

[66] A G Smith. "Embryo-derived stem cells: of mice and men". en. In: Annu. Rev. Cell Dev. Biol. 17 (2001), pp. 435-462. ISSN: 1081-0706. DOI: 10.1146/annurev .cellbio.17.1.435.

[67] E E Storm and D M Kingsley. "GDF5 coordinates bone and joint formation during digit development”. en. In: Dev. Biol. 209.1 (May 1999), pp. 11-27. ISSN: 0012-1606. DOI: 10. 1006/dbio.1999.9241.

[68] Christina J Su et al. "Ligand-receptor promiscuity enables cellular addressing". In: In preparation (2020).

[69] Elif Tekin et al. "Enhanced identification of synergistic and antagonistic emergent interactions among three or more drugs". en. In: J. R. Soc. Interface 13.119 (June 2016). ISSN: 1742-5689, 1742-5662. DOI: 10.1098/rsif.2016.0332.

[70] Peter Ten Dijke et al. "Identification of type I receptors for osteogenic protein-1 and bone morphogenetic protein-4”. In: J. Biol. Chem. 269.25 (1994), pp. 16985-16988. ISSN: 00219258.

[71] Anne L Theilmann et al. "Endothelial BMPR2 Loss Drives a Proliferative Response to BMP (Bone Morphogenetic Protein) 9 via Prolonged Canonical Signaling”. en. In: Arterioscler. Thromb. Vasc. Biol. (Oct. 2020), ATVBAHA119313357. ISSN: 1079-5642, 1524-4636. DOI: 10.1161/ATVBAHA. 119.313357

[72] Valer et al. ACVRI Function in Health and Disease. 2019. Dor: 10.3390/cells8111366.

[73] Elvira Valera et al. "BMP-2/6 heterodimer is more effective than BMP-2 or BMP-6 homodimers as inductor of differentiation of human embryonic stem cells". en. In: PLoS One 5.6 (June 2010), e11167. ISSN: 1932-6203. DOI: 10.1371/journal .pone.0011167.

[74] Folkert Verkaar and Guido J R Zaman. “A model for signaling specificity of Wnt/Frizzled combinations through co-receptor recruitment”. en. In: FEBS Lett. 584.18 (Sept. 2010), pp. 3850-3854. ISSN: 0014-5793, 1873-3468. DoI: 10.1016/j . febslet . 2010.08.030.

[75] Anna H C Vlot et al. "Applying synergy metrics to combination screening data: agreements, disagreements and pitfalls”. en. In: Drug Discov. Today 24.12 (Dec. 2019), pp. 2286-2298. ISSN: 1359-6446, 1878-5832. DOI: $10.1016 / \mathrm{j}$.drudis.2019.09.002.

[76] Alexander Weiss and Liliana Attisano. "The TGFbeta superfamily signaling pathway". en. In: Wiley Interdiscip. Rev. Dev. Biol. 2.1 (Jan. 2013), pp. 47-63. ISSN: 1759-7684, 1759-7692. DOI: $10.1002 / \mathrm{wdev} .86$.

[77] Ken Yamauchi, Keith D Phan, and Samantha J Butler. "BMP type I receptor complexes have distinct activities mediating cell fate and axon guidance decisions". en. In: Development 135.6 (Mar. 2008), pp. 1119-1128. ISSN: 0950-1991. DOI: 10.1242/dev.012989.

[78] Pamela Yeh, Ariane I Tschumi, and Roy Kishony. "Functional classification of drugs by properties of their pairwise interactions". en. In: Nat. Genet. 38.4 (Apr. 2006), pp. 489-494. ISSN: 1061-4036. DOI: $10.1038 / \mathrm{ng} 1755$. 
[79] Y Ying, X Qi, and G Q Zhao. "Induction of primordial germ cells from murine epiblasts by synergistic action of BMP4 and BMP8B signaling pathways". en. In: Proc. Natl. Acad. Sci. U. S. A. 98.14 (July 2001), pp. 7858-7862. ISSN: 0027-8424. DOI: 10. 1073/pnas . 151242798

[80] Paul B Yu et al. Bone Morphogenetic Protein (BMP) Type II Receptor Deletion Reveals BMP Ligand-specific Gain of Signaling in Pulmonary Artery Smooth Muscle Cells. 2005. DOI: 10.1074/jbc.m502825200.

[81] Zhike Zi, Douglas A Chapnick, and Xuedong Liu. "Dynamics of TGF- $\beta /$ Smad signaling”. en. In: FEBS Lett. 586.14 (July 2012), pp. 1921-1928. ISSN: 0014-5793, 1873-3468. DOI: $10.1016 / \mathrm{j}$. febslet.2012.03.063

[82] Anat Zimmer et al. "Prediction of multidimensional drug dose responses based on measurements of drug pairs”. en. In: Proc. Natl. Acad. Sci. U. S. A. 113.37 (Sept. 2016), pp. 1044210447. ISSN: 0027-8424, 1091-6490. DOI: 10.1073/pnas. 1606301113 
Chapter 3

\section{EXPANDING THE PREDICTIVE UNDERSTANDING OF BMP SIGNALING TO NEW COMBINATIONS AND CONTEXTS}

\subsection{Summary}

The large survey of BMP pairwise interactions across receptor contexts produced BMP "equivalence groups" that are useful for explaining some of the context-dependent differences in ligand behavior observed in development. However, it is easy to find the boundary of the usefulness of these equivalence groups. For example, they cannot predict the behavior of these ligands in any receptor context, lacking either the additional data or the fully-descriptive model required to do so. The equivalence groups do not show directly what physical differences between the ligands produce their unique effects. They also neglect many of the other proteins that can activate Smad1/5/8 or divert Smad1/5/8 activity into other outputs, differences that can also produce cell-type specific responses to BMP. Finally, they do not show how control of pathway activity affects the output we would most like to control: cell fate.

This chapter outlines initial attempts and future experiments to address these questions, which are divided into two groups. First, the mathematical model of BMP signaling provides a tool for predicting pathway activation in arbitrary contexts and possibly also for inferring the underlying ligand differences that produce these effects. Fitting the model to a larger dataset improves predictive power, but does not completely match experimentally observed behavior. We therefore explore the relative merits of collecting further data, trying an alternative model architecture, or measuring some biochemical parameters directly (Section 3.2). Second, the current experimental schema focuses on narrowly-defined combinations of certain ligands in few receptors contexts controlling a limited number of outputs. We therefore describe biologically relevant and experimentally accessible extensions of this system, such as studying combinations of more than three ligands, including related ligands that can also activate Smad1/5/8, perturbing other features of cell context, and comparing equivalence groups with control of cell fate (Section 3.3.

\subsection{Limitations of existing BMP model require more data or alternative model architecture.}

Ideally, the mathematical model of BMP signaling can be both predictive and explanatory, predicting BMP pathway activation in arbitrary contexts and explaining how these diverse cell responses emerge. However, extensive measurements of pairwise BMP interactions across seven cell types were not sufficient to constrain the many parameters required for the full model (Section 2.3.8). 
Many different parameter sets fit the data equally well (Figure $\mathrm{S} 2.8 \mathrm{~B}$ ), and even these top parameter sets did not match all experimentally observed interactions precisely (Figure S2.8A). Therefore, to improve model predictiveness, we generated new parameter estimates with additional data (Section 3.2.1), identified lingering problems with these fits by identifying the most poorly-constrained parameters (Section 3.2.2), asked if the model architecture makes certain parameters harder to estimate than others (Section 3.2.3), and determined which additional data are more likely to improve parameter estimates (Section 3.2.4). We also explore the possibility of a more complex but physically accurate model (Section 3.2.6) and directly measuring, instead of inferring, some model parameters (Section 3.2.7).

\subsubsection{Data collected at saturating ligand concentrations improves quality of fits but does not capture all pairwise interactions.}

The initial parameter estimates for the BMP model (Figure $\mathrm{S} 2.8 \mathrm{~B}$ ) could not predict pathway activation at concentrations other than the single concentration used for the majority of the pairwise measurements (Figure 3.1B). This suggested that dose response data, which captured pathway activity for single ligands at a range of concentrations, could further constrain model parameters. We therefore repeated the parameter fitting procedure described in Section 2.3.8, but added the dose response data (as in Figure S2.1D) to the pairwise data. Using the same criteria as described previously, we found nine top fits that had both low error overall and preserved important pairwise interactions (Figure S3.1A). This improved the quality of fit overall (Figure 3.1A), largely due to better predictions of pathway activation at subsaturating and supersaturating concentrations. Surprisingly, the new parameter estimates appear to be subsets of the original parameter estimates (Figure S3.1B,C). However, some pairwise responses remained poorly fit, with erroneous predictions of weak antagonism or synergy (Figure $3.1 \mathrm{C}$ ). Nonetheless, these errors are equivalent to discrepancies between the data and model fit on pairwise data only (Figure 3.1A).

Our inability to find even one parameter set that is consistent with all pairwise interactions may suggest this model does not capture the true mechanism of BMP signaling, and at least one option for improving the model's physical accuracy is discussed later (Section 3.2.6). However, our 65parameter model also has a relatively large volume of parameter space to explore, and this parameter space is littered with many local optima. Therefore, it seems reasonable that we simply have not found the right parameter estimates for this model. If so, additional data could reduce the number of local optima and increase the probability of identifying the true parameter values. 


\subsubsection{Diverse paraemter estimates fit the data equally well.}

Operating under the assumption that it is difficult to estimate the model parameters because we have insufficient data (rather than an ill-formed model), we sought to understand the nature of the uncertainty in the existing parameter estimates. The large differences between top parameter estimates may indicate that the model fit is insensitive to some parameters or represent distinct, non-overlapping parameter sets that fit the data equally well (Figure 3.2A). If the goodness of fit is insensitive to the value of certain parameters, these poorly-constrained parameters then gain priority when planning future measurements or reveal how the model fails to capture the data in a systematic way. Conversely, if the top parameter estimates are very different from each other, then the all parameters in general will benefit from collecting additional data. 
A

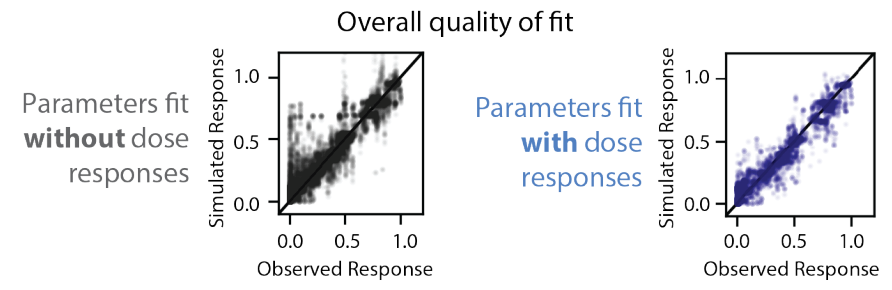

B

Sample dose responses (BMP9, four cell lines)
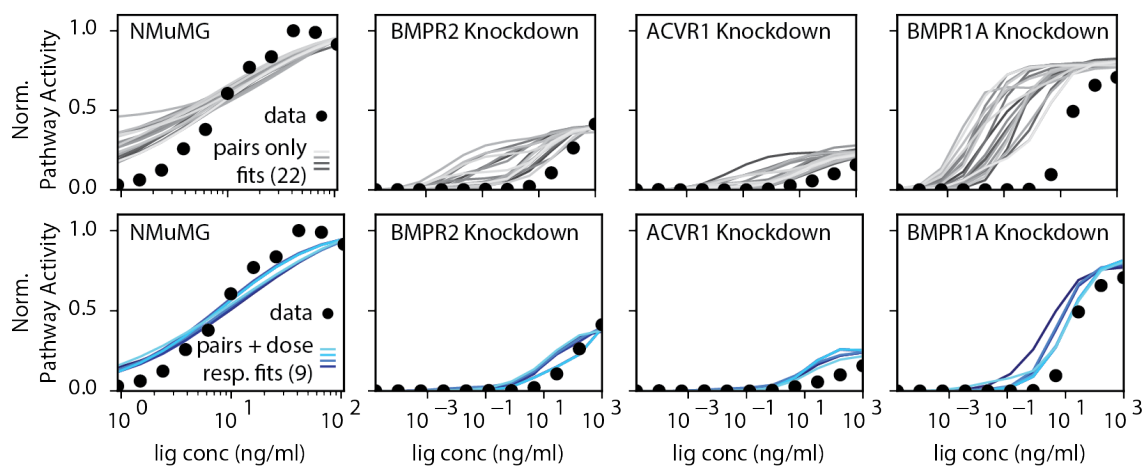

C

Poorly predicted pairs (using pairs+dose response fits)
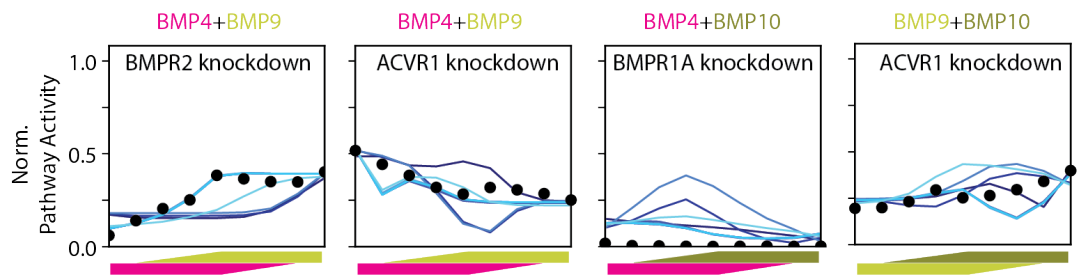

Figure 3.1: Fitting dose responses alongside rim data improves model performance at subsaturating ligand concentrations.

(A) Plotting the observed response against the predicted (i.e. simulated) response for all 970 datapoints shows that adding the dose responses to the parameter fits improves quality of fit overall. (B) A sample dose response for BMP9, measured in four different cell lines (NMuMG, BMPR2 knockdown, ACVR1 knockdown, BMPR1A knockdown; corresponding to four columns of plots) is better fit by the parameters generated by fitting rims and dose responses simultaneously (blue lines, second row) than fitting rims alone (gray lines, first row). While the gray fits produce the correct saturating level, they have highly variable $E C_{50}$ concentrations. Different numbers of "top" parameter fits are shown because different numbers of parameter sets met the criteria for being a top fit (i.e. low error overall and sufficiently strong imbalance and balance interactions).

(C) Despite the improvements in overall fit (panel $\mathbf{A}$ ) and in the dose responses (panel B), the new parameter estimates produce poor fits for some of the pairs. Examples of these problematic pairs are shown, which indicate weak but erroneous antagonism or synergy. Each subplot shows the response to a given BMP pair (title) in a specific cell line (inset title). Pathway activity is normalized between 0 and 1 after pooling all datasets. The $\mathrm{x}$-axis samples different ratios of the two ligands, as indicated by the heights of the trapezoids, whose color corresponds to one of the ligands in the pair.

See also Figure S3.1. 
A
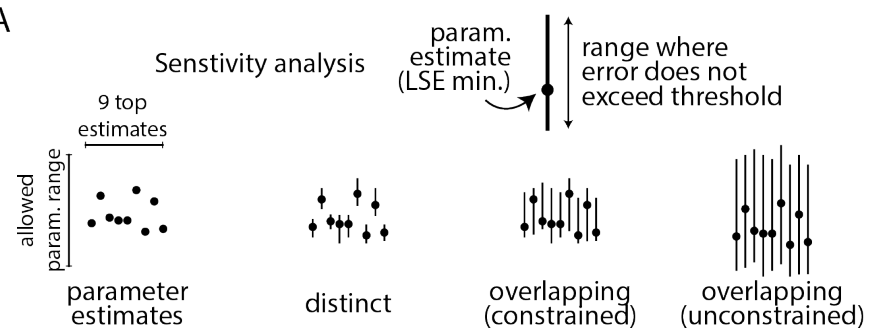

B

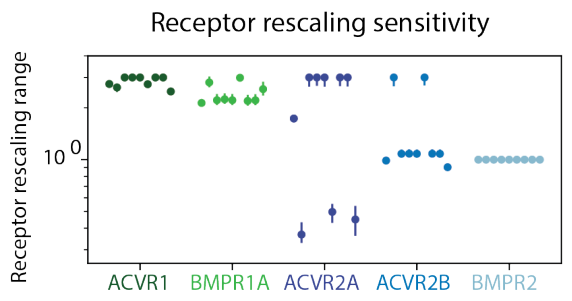

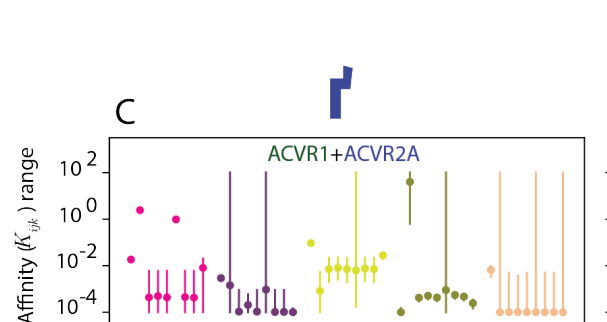

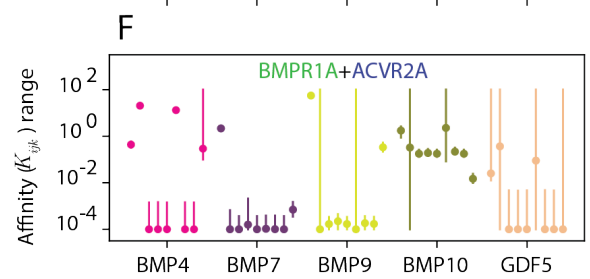

BMP4 BMP7 BMP9 BMP10 GDF5

Affinity $\left(K_{i j k}\right)$ sensitivity
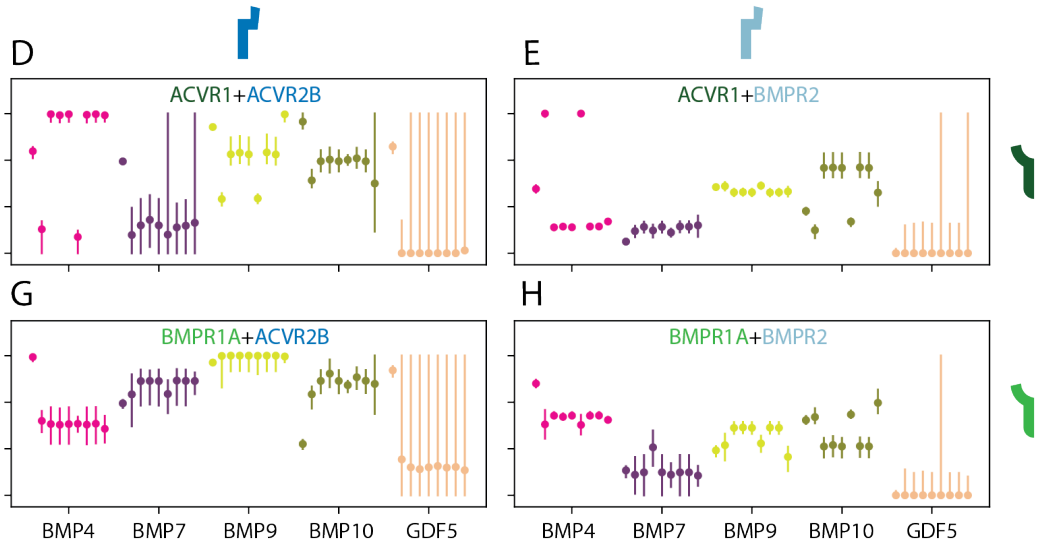

Activity $\left(\varepsilon_{i j k}\right)$ sensitivity
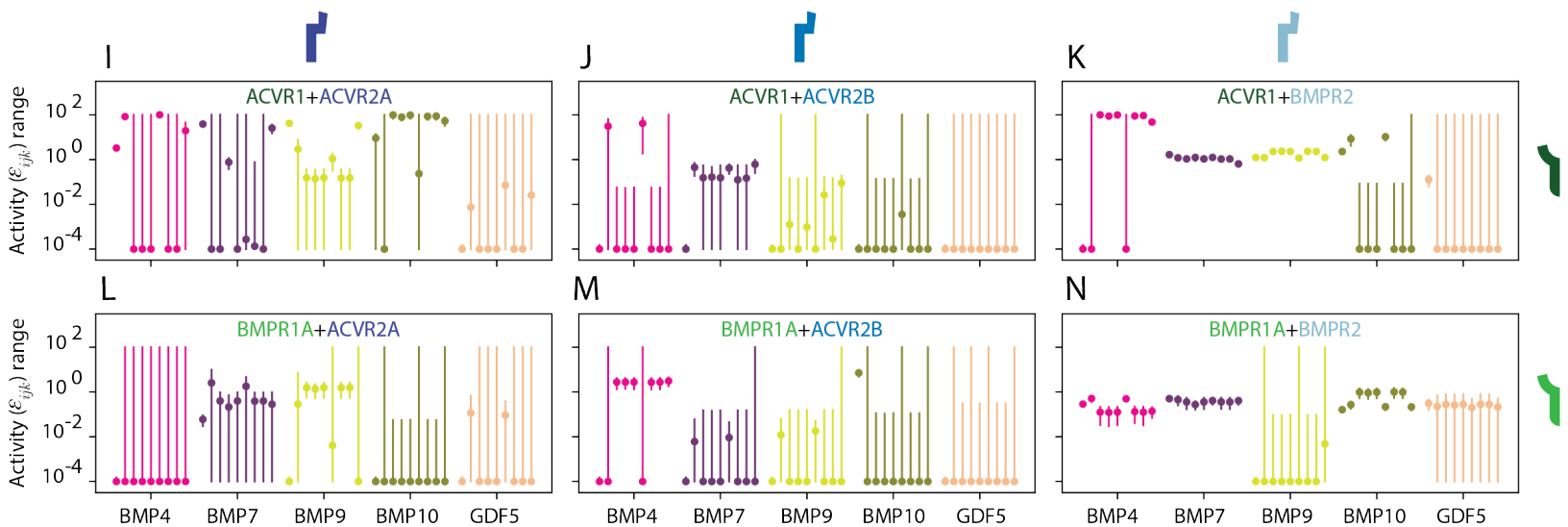

Figure 3.2: Parameter fits are more sensitive to receptor rescaling ratios and affinities than activities. (caption on following page) 
Figure 3.2 (previous page):

(A) Each parameter fit (dot) was varied to find the range over which the goodness-of-fit did not exceed the threshold for top parameter fits (line). The sensitivities for each parameter are plotted side-by-side for all nine top fits (shown in Figure $[\mathrm{S3.1B}$ ), to show if they represent distinct or overlapping solutions. The range of insensitivity (shown by the line) indicates how well the data constrain this parameter.

(B) Though receptor mRNA levels can be measured by qPCR, the actual protein levels may vary. This difference in receptor expression is captured with a receptor rescaling parameter. This parameter is limited between $\frac{1}{3}$ and 3 , with the exception of BMPR2, whose receptor rescaling parameter is fixed at 1. For all parameter sets, the goodness of fit is very sensitive to the values of these parameters. Also, there is good agreement between all fitting results that ACVR1 and BMPR1A should have higher values than estimated by qPCR.

(C-H) The goodness of fit is less sensitive to the affinity $\left(K_{i j k}\right)$ values. However, the degree of sensitivity varies with specific ligands and receptors. For example, the goodness of fit is largely insensitive to any GDF5 parameters (and some BMP7 parameters). By contrast, affinities for BMPR2-containing complexes (panels $\mathbf{E}$ and $\mathbf{H}$ ) seem best constrained by the data. Note that these parameters are arbitrarily restricted to values between $10^{-4}$ and $10^{2}$. Interestingly, some values do appear to have similar values across all fits (e.g. the affinity of BMP7 and BMP9 for BMPR1A+ACVR2B, purple and yellow in panel $\mathbf{G}$, or ACVR1+BMPR2, purple and yellow in panel E).

(I-N) The goodness of fit is largely insensitive to the activity $\left(\epsilon_{i j k}\right)$ values, with parameters falling into one of three groups. Some parameters can take any value in the allowed range $\left(10^{-4}-10^{2}\right)$, such as GDF5's activity through ACVR1+ACVR2A (orange, panel I). Others must take sufficiently low values, such as most of BMP7's activities through BMPR1A+ACVR2B being less than one (purple, panel M). A minority may take only a narrow range of values, mostly for BMPR2-containing complexes (panels $\mathbf{K}$ and $\mathbf{N}$ ).

To distinguish these two possibilities, we systematically and individually varied each of the parameters across the top nine parameter fits (as shown in blue in Figure $3.1 \mathrm{~B}$ ). We identified the maximum and minimum values each parameter could take while keeping the goodness-of-fit error below the threshold required to be considered a "top" fit (Figure 3.2). This revealed that the top parameter fits do indeed represent distinct solutions, as the regions of allowed parameter values for the nine top fits are non-overlapping in some cases. Thus, the uncertainty for certain parameters (e.g. BMP4's affinity for most receptor dimers, Figure $3.2 \mathrm{C}-\mathrm{H}$ ) arises because many different values can fit the data equally well. However, some parameters do take similar values in all top parameter fits, such as BMP7's and GDF5's affinity for BMPR1A+ACVR2B (Figure 3.2G). However, while the values for BMP7 are relatively well-constrained (falling between $10^{0}$ and $10^{1}$ ), those for GDF5 are not, as nearly any value can fit the data equally well.

The sensitivity analysis also revealed certain patterns in which parameters are constrained. For example, the goodness of fit is highly sensitive to the receptor rescaling parameters (Figure 3.2B) 
but largely insensitive to the activity parameters (Figure 3.2 I-N). In general, parameter estimates related to BMPR2-containing complexes are tightly constrained (Figures 3.2 E,H,K,N, rightmost column), whereas others are not. By contrast, nearly all the GDF parameters (orange in Figures $3.2 \mathrm{C}-\mathrm{N}$, rightmost of each subplot) are poorly constrained and can take any value. Lastly, in some cases where parameters take distinct values, they co-vary with other parameters. For example, the low values for ACVR2A rescaling occur when ACVR2B rescaling is high and vice versa (Figure 3.2B), perhaps indicating that, in addition to being sensitive to receptor rescaling ratios, all fits require that either ACVR2A or ACVR2B be rescaled to a higher value, but not both.

Thus, the nine estimates of parameter values represent different parameter configurations that fit the data equally well. This makes the case for generally collecting more data to distinguish between these top solutions. However, the existence of overlapping and unconstrained parameters also reveal that the goodness-of-fit is largely insensitive to some parameter values. Key examples are most GDF5 parameters (orange in Figures $3.2 \mathrm{C}-\mathrm{N}$ ) and the activities of signaling complexes containing ACVR2A and ACVR2B (Figures 3.2I,J,L,M). This may indicate something in the structure of the data or the parameters themselves that is challenging to fit. For example, GDF5 does not strongly activate any of the cell lines used for fitting the parameters, which may make its parameters largely irrelevant to predicting overall pathway activation. Similarly, ACVR2A and ACVR2B are the two most lowly expressed receptors, so can perhaps take on very different values without changing the overall goodness of fit. Alternatively, ACVR2A and ACVR2B are the only receptors that were not perturbed, i.e. present at two different levels in the dataset. We first explore, using a simpler version of the BMP model, whether some parameters are harder to estimate than others, by virtue of taking more extreme values (Section 3.2.3). With these limitations in mind, we next ask which data would most likely decrease our uncertainty for the most poorly constrained parameters (Section 3.2.4).

\subsubsection{In analytical model, extreme receptor ratios or very low affinities are harder to esti- mate.}

To determine if the structure of the data or the parameter values themselves place intrinsic constraints on parameter estimation, we need examples of estimating BMP model parameters when the true parameter values are known. Fitting data simulated using known ground truth parameters allows direct comparison between between parameter estimates and their true values. Therefore, we simulated data from a simpler version of the BMP model containing only one ligand, one Type I receptor, and one Type II receptor (Figure 3.3A). This model has fewer parameters, so gradient descent, with a smaller volume of parameter space to explore, terminates more quickly and permits the analysis of more "ground truth" parameter sets. Data were simulated with multiplicative Gaussian noise from 100 random parameter sets, and the noisy data from each parameter set were 
fit by multiple rounds of gradient descent (Figures 3.3B,C). The "best" fit parameter estimate was the gradient descent solution that produced the lowest least squared error (LSE) between the model and the simulated data. For nearly $90 \%$ of the random "ground truth" parameter sets, the "best" fit parameter estimates were within 2-fold of the true value (Figure 3.3D). Among the three fit parameters, $k^{*}$ was estimated most accurately, being within 2-fold error for 98 of 100 parameter sets.

Note that the very high quality of the estimates here, as well as the agreement between the many gradient descent solutions for some parameters (as in Figure 3.3 C), are not necessarily the expected behavior when fitting the more complicated model used for the real dataset, which contains five ligands, two Type I receptors, and three Type II receptors. Rather, within this simple model, we compared parameter sets which were fit with higher and lower error overall to see what features, if any, made them more challenging to estimate than others. Within data simulated from the same model architecture, two kinds of parameter values produced less accurate parameter estimates. 
A

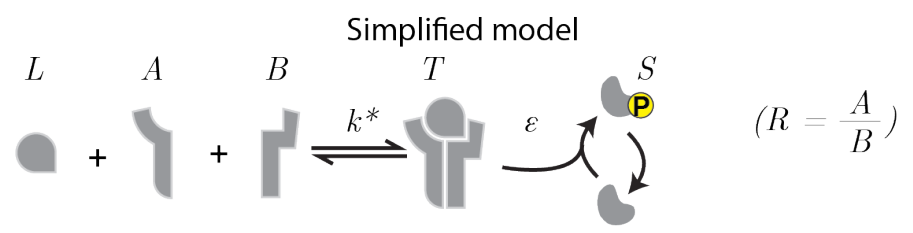

B

Distribution of simulated parameters
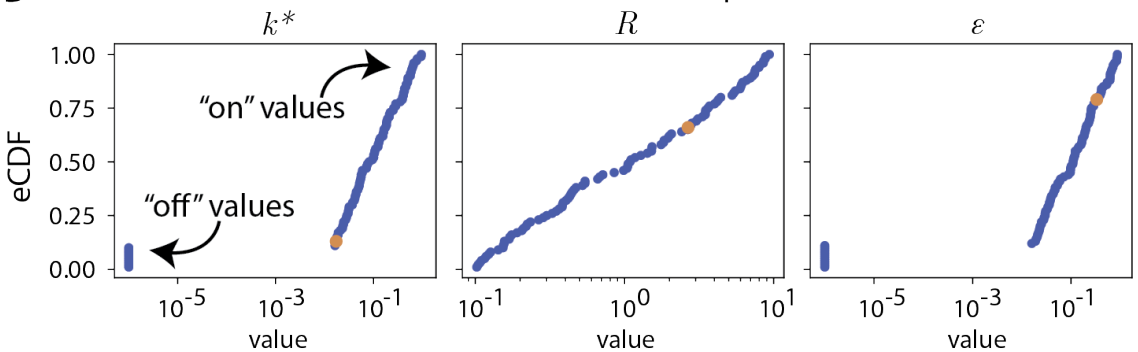

C

Sample gradient descent solutions (one parameter set)
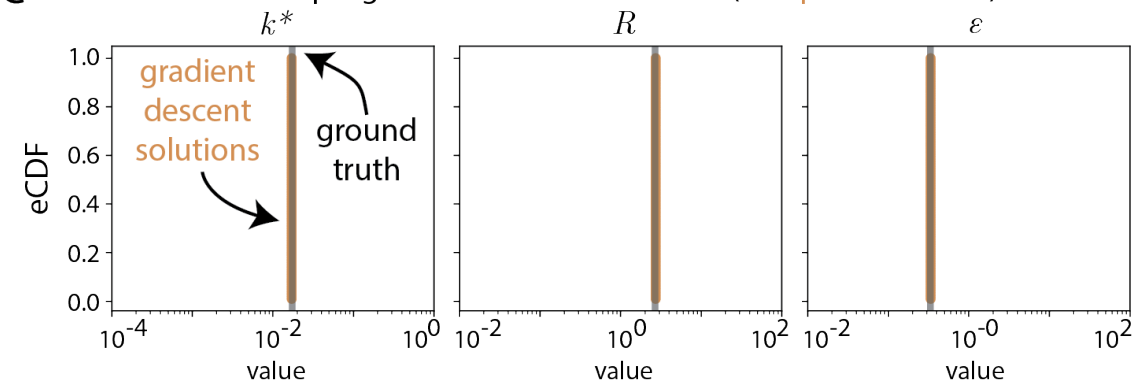

D

Error of best fit parameter estimate

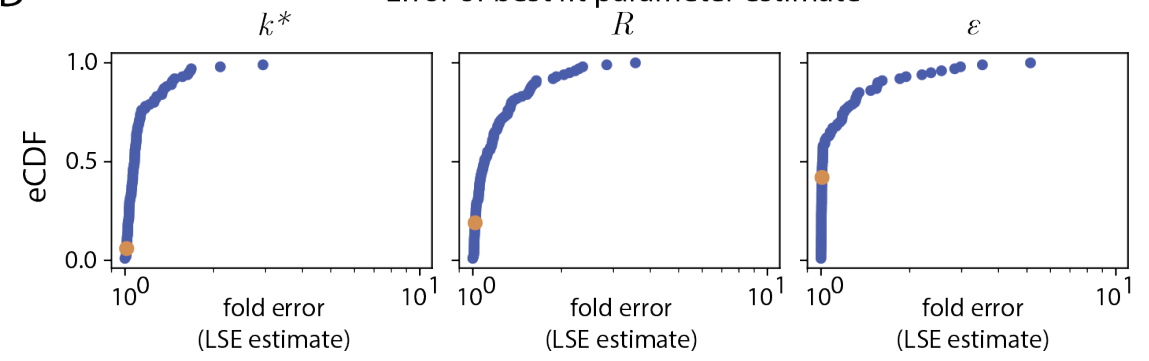

Figure 3.3: Parameters of simple one-step model can be fit within 2-fold error for nearly all parameter sets. (caption on following page) 
Figure 3.3 (previous page):

(A) To generate data with known ground truth parameters, data were simulated with a simpler version of the original BMP model (Figure 2.7A), containing only one ligand, one Type I receptor, and one Type II receptor. The fully non-dimensionalized model includes three parameters: $k^{*}$, the affinity of signaling complex formation; $\epsilon$, the activity of the resulting signaling complex; and $R$, the ratio of receptor expression levels. To make these simulations more like the data we collect, multiplicative Gaussian noise (mean $=1$, std $=0.1$ ) was added to the data. Similarly, multiplicative Gaussian noise ( mean $=1$, std $=2$ ) was added to the estimate of receptor expression, and $R$ was fit as an estimate of this noise.

(B) 100 ground truth parameters were selected log-normal over two orders of magnitude, an estimate of the allowed variation in biochemical parameters. To simulate the presence of rare "non-activating" ligands that either do not bind or do not activate receptors, "off" parameter values $\left(10^{-6}\right)$ were added to some parameter sets. One sample parameter set is highlighted in beige.

(C) To estimate parameters, we used gradient descent to minimize least squared error between the simulated data and the model. For each parameter set, we produced 100 gradient descent solutions from 100 different initial guesses. Shown here are the 100 solutions for the sample parameter set highlighted in $\mathbf{B}$, which are all very close to the true parameter values.

(D) A "best" parameter estimate was chosen for each parameter set by selecting the one of the 100 gradient descent solutions that produced the lowest least squared error overall. The fold error between this best estimate and the true value are shown for all 100 parameter sets from $\mathbf{B}$, with the beige dot highlighting the same parameter set as in $\mathbf{B}$ and $\mathbf{C}$. For $k^{*}$, all but two of the parameters (e.g. 98\%) are within 2-fold of the true value. For $R$ and $\epsilon$, the best estimate was within 2-fold of the true value for close to $90 \%$ of parameter sets.

First, very low affinity $\left(k^{*}\right)$ values produced less accurate estimates of $k^{*}$ and $\epsilon$ (Figure 3.4A). Low affinity $\left(k^{*}\right)$ and activity $(\epsilon)$ values were inserted into the randomly generated sets (Figure $3.3 \mathrm{~B}$ ) to match the infrequent occurrence of non-activating ligands in our dataset (Figure S2.1D), which either have very low affinity for the receptors or very low activity. However, only low affinity values decreased the quality of parameter estimates. Intuitively, this difference may arise because of differences in how affinity versus activity affect the dose response curve shape. A change in affinity translates the Hill function, such that the $E C_{50}$ occurs at lower or higher ligand concentrations (Figure S3.2A), whereas activity rescales the amplitude of the Hill function (Figure S3.2C). The dose response data are simulated on a fixed ligand concentration range, mirroring the collection of experimental data at ligand concentrations bounded by the cost and feasibility of obtaining very high concentration protein stocks. Thus, very low $k^{*}$ values can "translate" the informative part of the Hill function outside the simulated concentration range, making it indistinguishable from a similar parameter set with different affinity and receptor expression values (Figure $\mathrm{S} 3.2 \mathrm{~B}$ ). By contrast, a very low-amplitude Hill function may be approximated with a higher activity and lower affinity ligand, but only a low activity, high affinity ligand produces the lowest error fit. Thus, the "true" solution can be distinguished even among a wide variety of parameter estimates produced 
by different gradient descent runs (Figure S3.2D). Overall, this shows that while gradient descent may produce a variety of parameter estimates, it is possible to correctly parameterize a low-activity ligand, while it is harder to do so for low-affinity ligands, which influence a portion of the dose response that may require extremely high protein concentrations to access.

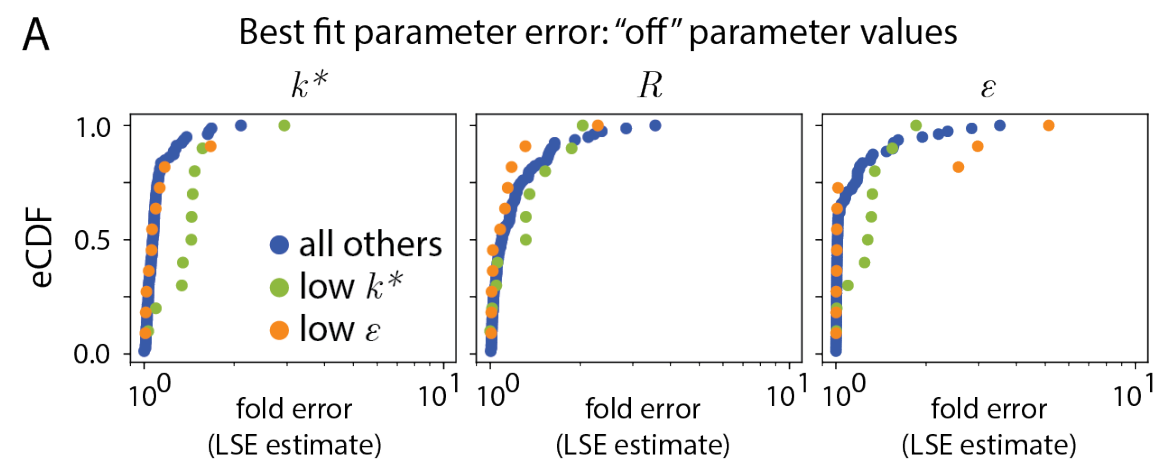

B Best fit parameter error: extreme receptor ratios

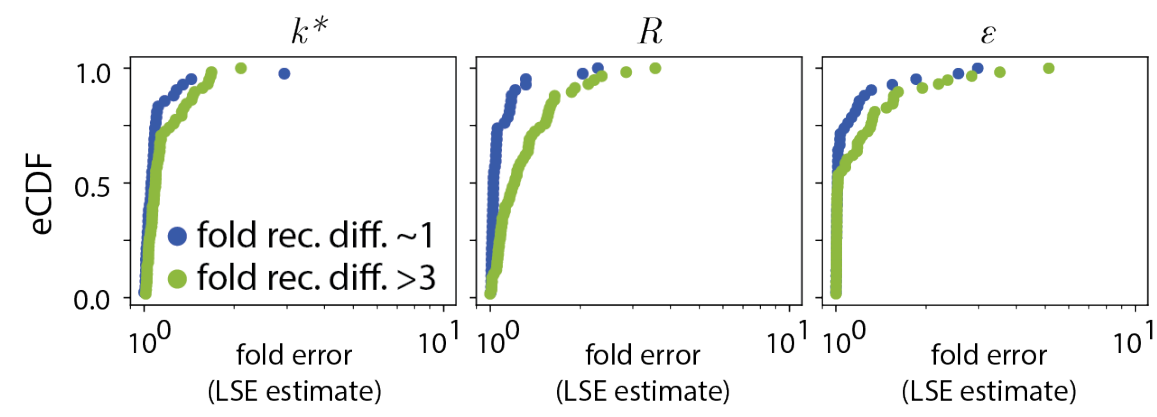

Figure 3.4: Very low affinities and more extreme receptor ratios produce less accurate parameter estimates. Binning the 100 parameter estimates by underlying features of the parameters reveals differences in their best estimate error. Best fit parameter estimates were those that minimized the least-squared error (LSE) between the simulated data and model prediction.

(A) If a parameter set contained a very low affinity (i.e. $k^{*}=10^{-6}$ ), best fit parameter estimates had higher errors than in other simulations. By contrast, parameter sets with low activity ligands (i.e. $\epsilon=10^{-6}$ ) were estimated with similar error as other parameter sets.

(B) Parameter sets containing less extreme receptor ratios (i.e. $\frac{1}{3}<\frac{A}{B}<3$ ) were estimated with lower fold error than those with extreme receptor ratios. All parameters were affected, though $R$ has the largest increase in fold error.

See also Figure $\mathbf{S 3 . 2}$

The second hard-to-estimate parameter was a more extreme receptor ratio, where the amount of Type I receptor was very different from the amount of Type II receptor. Parameter sets with more extreme receptor ratios were estimated with higher fold error for all three parameters, but with the largest error in $R$ itself (Figure 3.4 $\mathrm{B}$ ). This may occur because the ligand is provided in excess, and either one of the two receptors will be limiting, which sets an upper bound on the number of 
signaling complexes that can form. If one receptor vastly outnumbers the other (i.e. the receptor ratio is large), small differences in how much that receptor outnumbers the other receptor produce a similar absolute number of signaling complexes. Therefore, precisely estimating the receptor ratio could be challenging if one receptor is in significant excess. Additionally, other parameter estimates show that a small error in the estimate of $R$ can be offset by an error in $k^{*}$, allowing additional parameter combinations to fit the data equally well.

While the 1-1-1 model is very different from the 5-2-3 model fit in Chapter 2, these results provide evidence that the values of the parameters themselves may make them harder to fit, and the trends shown here are consistent with specific challenges we encountered in fitting the 5-23 model. In particular, ligands will low affinity and, in some cases, low activity are harder to parameterize. Indeed, the sensitivity analysis revealed that our top fits were relatively insensitive to values selected for GDF5, a non-activating ligand (Figure 3.2). Extreme receptor ratios may also make all parameters harder to fit. When fitting the experimental data, parameters for ACVRB were also poorly constrained, and ACVR2B is expressed 3-fold lower than nearly all other receptors in wildtype NMuMG (Figure 2.4A).

These results have important implications for collecting data to constrain model parameters. First, accurately identifying GDF5's low affinity or low activity may require alternative experimental conditions that make that low affinity or activity apparent. For example, significant overexpression of the receptor that it weakly binds could produce a measurable response at lower ligand concentrations. Alternatively, if GDF5's weak binding is sufficient to inhibit the activity of other ligands (e.g. its antagonism of BMP4, cf. Figure 2.3 C), pairwise interactions may be more informative than dose responses. However, outside these two options, it may not be feasible to infer GDF5's weak binding and activity parameters for pathway activation data. By contrast, the receptor ratio $R$ can be directly controlled in the experiment. This analysis suggests that collecting data when a receptor vastly outnumbers the others or is itself vastly outnumbered make it challenging to estimate any of the model parameters. Therefore, overexpressing ACVR2A and ACVR2B in the BMP reporter cell line may be the perturbation most likely to constrain the remaining model parameters.

\subsubsection{Ligand combinations and receptor perturbations constrain parameters more than individual dose responses or cell lines alone.}

While the 1-1-1 model (i.e. one ligand, one Type I receptor, one Type II receptor) highlights possible experiments that are more likely to constrain model fit parameters, the true 5-3-2 model (i.e. five ligands, two Type I receptors, three Type II receptors) that describes the data has more complicated behaviors. To further explore which data constrain the parameters most effectively, we fit the full 5-3-2 model on different subsets of the data and compared the overall range and median 
of these parameter estimates. If removing data significantly changed the the range or the median of the parameter estimates, we inferred that that the removed data were important for constraining that parameter. In this way, we can determine the relative importance of the single ligand and pairwise data, as well as the role of different receptor perturbations in constraining parameters for their related receptors.

\section{Pairwise measurements necessary to constrain most parameters.}

Theoretically, a given ligand's affinity and activity parameters can be fit with data where the ligand signals alone, rather than in pairs with other ligands. We therefore asked how the best fit parameter estimates varied when they were determined using single ligand dose responses, compared to when both the dose responses and pairwise measurements were used. As with the fitting the full dataset, fitting a subset produced a unique solution for each gradient descent run initiated with a different random parameter guess. Therefore, to find differences in parameter estimates, these distributions of gradient descent results were compared. Strikingly, fitting on dose response data alone increased the least-squared error 10-fold relative to models fit with both dose response and pairwise data. Thus, no parameter fits generated from dose responses alone fit the data as well as parameter fits generated from both dose responses and pairwise measurements. Further, roughly $\frac{2}{3}$ of the parameters were worse constrained when fit on gradient data alone, having either a 5fold larger range of middle 50\% fits or a median estimate that was 5-fold larger or smaller than the estimate generated by fitting all the data (Figure 3.5). Neither affinities, activities, or ligand-specific parameters were fit particularly well using gradient data alone. Overall, this suggests that pairwise data are necessary to constrain parameter estimates. This is perhaps because, as described above, low-affinity ligand receptor interactions are not apparent in the dose response data. Another likely cause is the presence of multiple co-expressed receptors, which, on average, makes it harder to correctly infer the behavior of the individual receptors.

\section{Receptor perturbation data improves parameter estimates for unrelated receptors.}

In parallel to the question of whether ligands signaling individually or in pairs provide the necessary data for constraining model parameters, we can also ask what receptor configurations most effectively constrain model parameters. Ideally, since the affinity $\left(K_{i j k}\right)$ and activity $\left(\epsilon_{i j k}\right)$ parameters describe the interactions of a single ligand with a receptor pair (i.e. one Type I receptor paired with one Type II receptor), each receptor pair would be studied in isolation. However, while ligand combinations are produced by mixing these proteins in the cell media, receptors must be expressed on the cell surface, and most cell lines express many BMP receptors simultaneously. Producing an 
Quality of parameter estimates without pairwise data
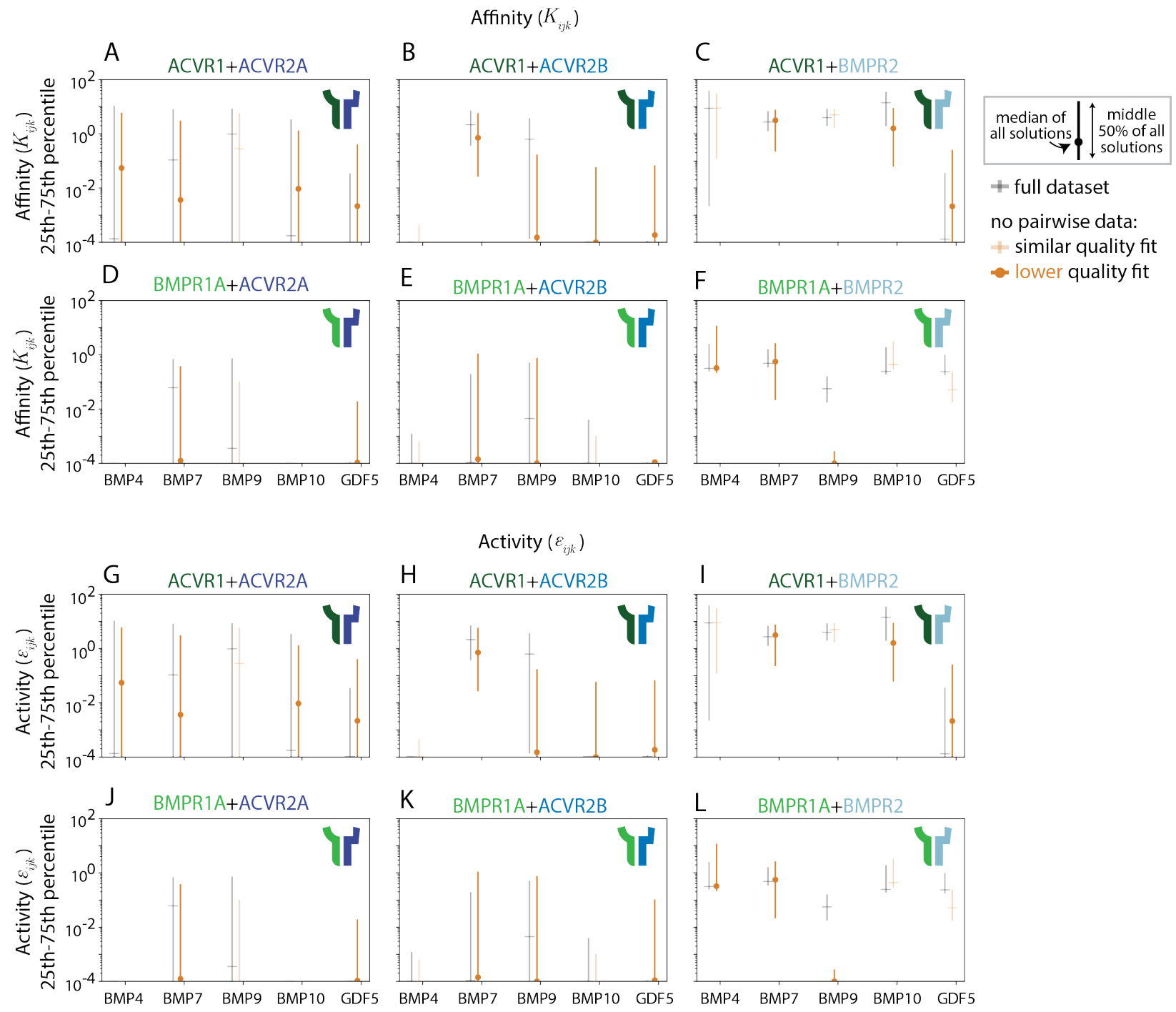

Figure 3.5: Pairwise data constrains parameter fits more than dose responses alone. Model parameters were fit using full dataset (4 cell lines, pairwise data and dose response data, 6000 gradient descent runs) or dose responses alone (4 cell lines, 200 gradient descent runs). The $25^{\text {th }}$ - $75^{\text {th }}$ percentile (line) and median (marker) of all gradient descent results are compared for the full dataset (gray) and fitting on dose responses only ("no pairwise data," orange). Roughly $\frac{2}{3}$ of parameters (38 of 60) have decreased quality of fit when fit on dose response data alone. Decreased quality of fit is highlighted with opacity and different marker style, where decreased quality of fit is a 5-fold difference in the median or 5-fold larger range between the $25^{\text {th }}$ and $75^{\text {th }}$ percentile. Affinity $\left(K_{i j k}, \mathbf{A}-\mathbf{F}\right)$ and activity $\left(\epsilon_{i j k}, \mathbf{G}-\mathbf{L}\right)$ are grouped by the distinct Type I and Type II receptor pairs.

arbitrary receptor combination therefore requires more time-intensive genetic engineering, which is not guaranteed to produce the desired effect. Therefore, rather than considering the ideal receptor configuration, we can restrict ourselves to considering the most accessible receptor configura- 
tions. These include either ectopic expression or knockdown of a single receptor in the NMuMG background, which expresses five of the seven BMP receptors (Figure 2.4A).

Quality of fit parameters without receptor perturbations
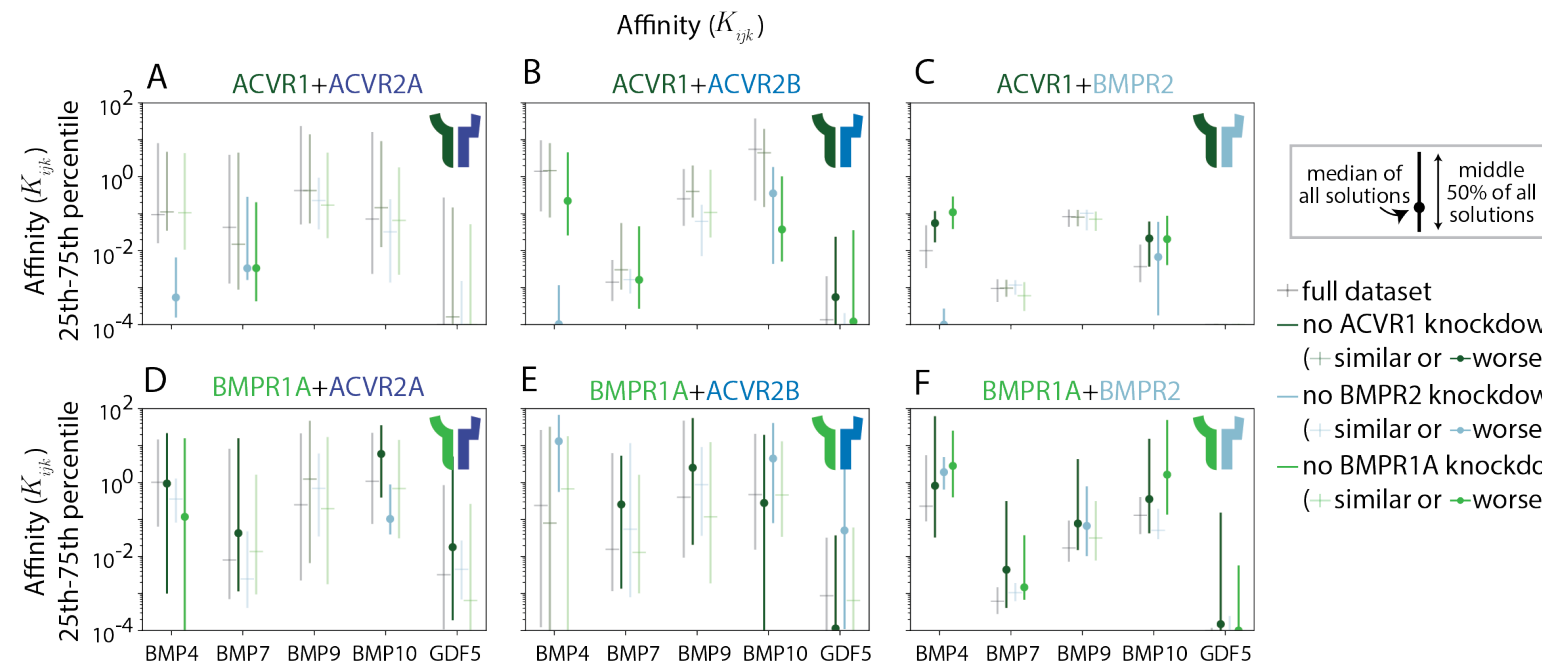

+ full dataset

- no ACVR1 knockdown data

( + similar or $\rightarrow$-worse fit)

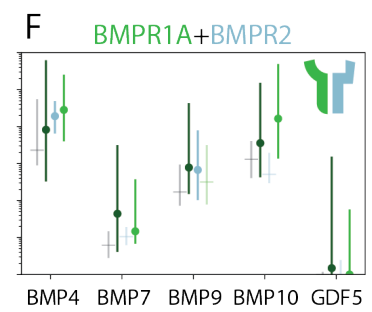

no BMPR2 knockdown data

( similar or - -worse fit)

- no BMPR1A knockdown data

( + similar or $\rightarrow$-worse fit)

Activity $\left(\varepsilon_{i \jmath k}\right)$
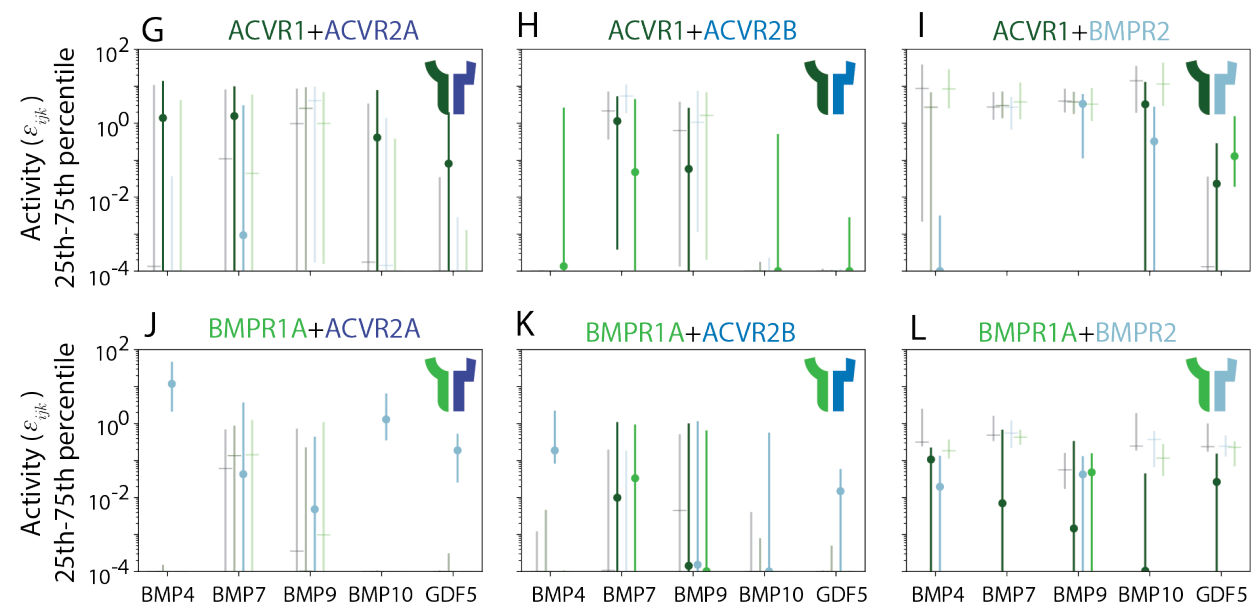

Figure 3.6: Receptor perturbation data constrains parameters for unrelated receptors. Model parameters were fit using full dataset (4 cell lines, 6000 gradient descent runs) or without one of the three knockdown cell lines ( 3 cell lines, 200 gradient descent runs for each set of 3 ). The $25^{\text {th }}$ - $75^{\text {th }}$ percentile (line) and median (marker) of all gradient descent results are compared for the full dataset (gray) and datasets lacking a specific receptor perturbation (knockdowns of ACVR1, BMPR2, or BMPR1A; dark green, light blue, or light green, respectively). Removing a receptor perturbation dataset decreases quality of fit for parameters concerning unrelated receptors, such as the importance of the ACVR1 knockdown for estimating most ligands' affinities for BMPR1Acontaining complexes (panels $\mathbf{D}, \mathbf{E}, \mathbf{F}$ ). Decreased quality of fit is highlighted with opacity and different marker style, where decreased quality of fit is a 5-fold difference in the median or 5-fold larger range between the $25^{\text {th }}$ and $75^{\text {th }}$ percentile. Affinity $\left(K_{i j k}, \mathbf{A}-\mathbf{F}\right)$ and activity $\left(\epsilon_{i j k}, \mathbf{G}-\mathbf{L}\right)$ are grouped by the distinct Type I and Type II receptor pairs. 
The analysis of the 1-1-1 model (Section 3.2.3) suggests that receptor parameters are wellconstrained when they are expressed in close to a 1:1 ratio with other receptors. This suggests that ACVR2A and ACVR2B parameters are harder to fit, as they are expressed at lower levels than all other receptors and never perturbed. By contrast, BMPR1A and BMPR2 parameters may be easier to estimate, as they are expressed in nearly a 1:1 ratio after ACVR1 is knocked down.

This intuition from the 1-1-1 model was consistent with the observed effects. Specifically, removing the ACVR1 knockdown from the dataset did not affect the estimates of ACVR1-related parameters, but significantly worsened estimates of BMPR1A-related parameters (Figures 3.6D,E,F). Thus, the ACVR1 knockdown data, a receptor configuration dominated by BMPR1A and BMPR2 expressed at high and equivalent levels, was most useful for constraining BMPR1A related parameters. Similarly, removing the BMPR1A knockdown affected estimates of ACVR1-related parameters more than BMPR1A-related parameters (Figures 3.6A,B,C). In the BMPR1A knockdown, the receptor configuration is dominated by ACVR1 and BMPR2 expressed at high and equivalent levels and was most useful for constraining ACVR1-related parameters. By contrast, the BMPR2 knockdown is not particularly useful for constraining most parameter estimates, perhaps because knocking down BMPR2 produces only a more extreme Type I to Type II receptor ratio.

Together, these results suggest that, in addition to requiring Type I and Type II receptors be expressed at similar levels (i.e. ratio close to 1 ), better parameter estimation requires both receptors to be more highly expressed than the other Type I and Type II receptor variants. This was not clear from the 1-1-1 model, where there was only one variant each of the Type I and Type II receptors.

\subsubsection{Future parameter fitting should focus on ACVR2A and ACVR2B overexpression, with possible BMPR2 knockdown.}

The best parameter estimates produced so far still include multiple, distinct parameter estimates (Figures S3.1 and 3.2). Our inability for to indirectly infer certain parameters seems likely to arise from both the biochemical values themselves and the types of data collected so far. GDF5's either low affinity or low activity may be hard to estimate no matter the ligand or receptor configuration tested (Figure S3.2A), since they do not produce significant changes in dose responses or pairwise data. However, the affinities and activities of ACVR2A- and ACVR2B-containing complexes may have little effect on the data only because these two receptors were lowly expressed relative to the other Type II receptor BMPR2 (Figure 3.6) and vastly outnumbered by the Type I receptors (Figure $\mathrm{S} 3.2 \mathrm{~B}$ ). Overexpressing ACVR2A and ACVR2B could provide a receptor configuration that better captures the effects of those receptors. Moreover, knocking down BMPR2 alongside these overexpressions may even better constrain the parameter estimates, by making ACVR2A or ACVR2B the dominant Type II receptor. 


\subsubsection{Two-step model provides an alternative and more physically accurate model architec-} ture.

The difficulty of finding best fit parameters for a one-step model of BMP complex formation (Figure 3.1) may emerge, as described above, from having too little data or certain parameters having harder-to-estimate values. However, it may also suggest that the one-step model is qualitatively not consistent with the data. Indeed, chemical reaction kinetics suggests that three-way interactions of components are highly improbable. Fitting the data may require a model of receptor complex formation that is more physically reasonable.

Realistically, the first binding step in BMP signaling complex formation can happen in one of three ways. The ligand can bind to a Type I receptor first, or to a Type II receptor, or the Type I and Type II receptor can form a complex. Co-immunoprecipitation studies suggest that all three complexes can be found, including, surprisingly, dimers of Type I and Type II receptors in the absence of recombinant BMP ligands. ${ }^{17}$ Moreover, though only a subset of all possible ligandreceptor affinities have been measured, individual ligands are observed to have order-of-magnitude higher affinities for either Type I or Type II receptors22/47/3/26 (Figure 3.7), suggesting that they will tend to bind in different orders from each other, binding either Type I or Type II in their first step.

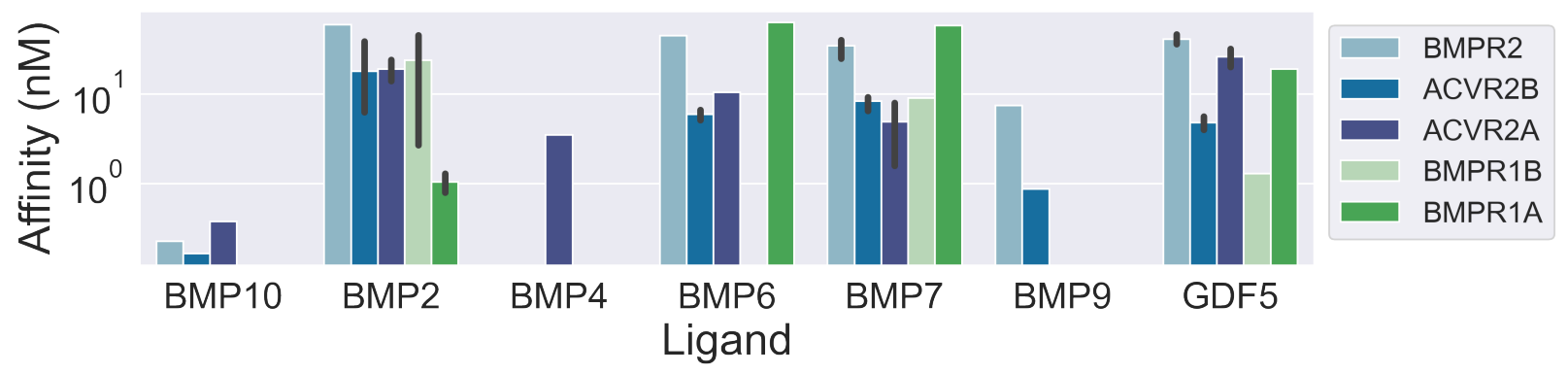

Figure 3.7: Published data show order of magnitude differences in ligand-receptor affinities. Data from [22, 47, 3, 26] shows that ligands can have highest affinity (i.e. lowest height bar) for Type I (green hues) or Type II (blue hues) receptors. For example, BMP2 has highest affinity for a Type I receptor (BMPR1A) whereas BMP6 and BMP7 have highest affinity for Type II receptors (ACVR2A and ACVR2B).

The reported variance in BMP ligand-receptor affinities and the existence of "pre-formed" receptor complexes indicate that a two-step BMP model should include all possible paths toward a full receptor complex. However, if all steps are to be in equilibrium with each other simultaneously, any route to producing the complex must occur at the same rate. This requirement of "detailed balance" indicates that the affinities of these various steps are not independent of each other. Taking these dependencies into account when specifying the model is non-trivial, so only a simple two-step model is shown here, where the only allowed first step is a ligand binding to a Type I receptor. 
Though not as accurate as a fuller two-step model, even a simple two-step model behaves differently from the one-step model in key aspects.

\section{Simple 1-1-1 model suggests two-step model may be hard to fit and to distinguish from one-step model.}

To compare the behavior of one-step and two-step models, we simulated noisy data from a simple two-step model (Figure 3.8 A) and compared best fit parameter estimates to the ground truth parameters. We followed the same procedure as in Section 3.2.3, except that we performed 1000 gradient descent runs per parameter set, as the larger number of parameters required exploring a larger volume of parameter space. Surprisingly, even in the simple case of one ligand, one Type I receptor, and one Type II receptor, best fit parameter estimates were considerably worse than in the one-step case (Figure $3.8 \mathrm{~B}$ ). The affinity of the second step in complex formation $\left(k_{2}^{*}\right)$ was particularly challenging to fit, with almost $75 \%$ of best fit parameter estimates have $\geq 2$-fold error. Even the best estimated parameter $\left(k_{1}\right)$ had $>10$-fold error in $10 \%$ of parameter estimates. The challenges of accurately fitting the two-step model suggest that more data will be necessary to constrain the affinities than for a one-step model. The simulated data were simple dose-responses in a single receptor context (i.e. only one value of $R$ ). Therefore, pairwise measurements, where ligands compete for shared receptors, or repeated dose responses in different receptor contexts, where $R$ takes on different values, may be necessary to constrain the harder-to-fit two-step model parameters, such as $k_{2}^{*}$ and $\epsilon$.

The possibility of fitting a two-step model raises the question of whether data generated from the two models can be distinguished. Though our minimization of least-squared error is consistent with a likelihood that assumes Gaussian-distributed error (of median 0 and homoscedastic standard deviation $\sigma$ for all data points) around the model prediction, we do not have an analytical expression of the likelihood that marginalizes out this $\sigma$ and is properly normalized as a probability distribution function. It is therefore difficult to implement rigorous model selection strategy, which quantitatively compares the error of a model fit with a penalty for model complexity.

Instead, we use the least squared error of our best fit estimates to approximate which model fits the data better, regardless of model complexity. We consider the one-step model to a better fit if $\frac{\text { One-step best fit LSE }}{\text { Two-step best fit LSE }} \leq 1$. Note that if the two models fit the data equally well ( $\frac{\text { One-step best fit LSE }}{\text { Two-step best fit LSE }}=1$ ), we would default to the less complex (i.e. one-step) model. Arbitrarily, we implement a threshold of 1.5 as the minimum fold decrease in least squared error in order to favor the more complex (i.e. two-step) model.

For $50 \%$ of the datasets generated from one-step models, fitting with a one-step model produces 
A

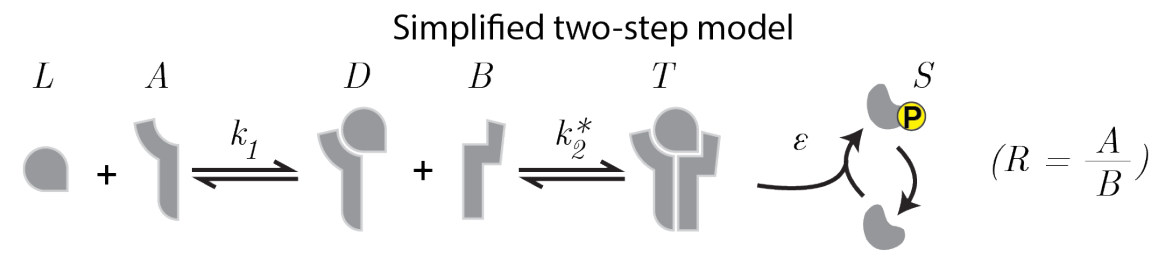

B

Error of best fit parameter estimate
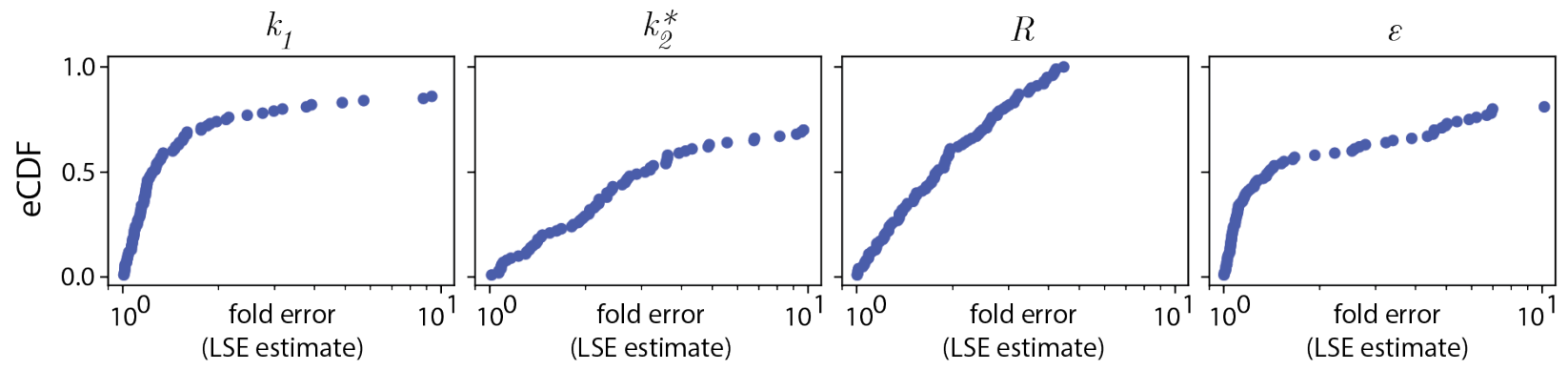

Figure 3.8: Best fit estimates of two-step model parameters have large errors.

(A) Relative to the one-step model (Figure 3.3A), the two-step model adds an additional equilibrated step in signaling complex formation. The ligand binds the Type I receptor forming a dimer $(D)$, which can then bind a Type II receptor to form the full trimer $(T)$. While fit parameters $R$ and $\epsilon$ are the same as in the one-step model, the parameter $k^{*}$ from the one-step model is replaced with two parameters: $k_{1}$ and $k_{2}^{*}$.

(B) For 100 randomly selected parameters, 1000 gradient descent solutions are produced from 1000 different initial conditions. The gradient descent solution with the lowest least squared error (LSE) overall is taken as the "best fit" and compared with the "ground truth" value to calculate the fold error in the parameter fitting. While the one-step model produced estimates within 2-fold of the real value for $90 \%$ of random, ground truth parameter sets, the two-step model does so for only $70 \%, 25 \%, 50 \%$, and $50 \%$ of the $k_{1}, k_{2}^{*}, R$, and $\epsilon$ parameter sets, respectively.

the lowest error fit overall. A further $45 \%$ of the datasets are only slightly improved by a two-step model, leaving only a minority of cases where a two-step model erroneously provides a significantly better fit than the one-step model. By contrast, in at least $75 \%$ the datasets generated from two-step models, the one-step model provides a better or equivalent fit with the two-step model. Though most two step data were not fit well and may have higher LSE as a result (Figure 3.8B), it is surprising to note that one-step and two-step models can produce similarly good fits of the data in many cases, whether the data was generated from an underlying one-step or two-step model. At best, these results indicate that great care should be taken in determining best fit parameters for a two-step model before performing model selection, or, at worst, the two models may be indistinguishable when collecting data in this way. 


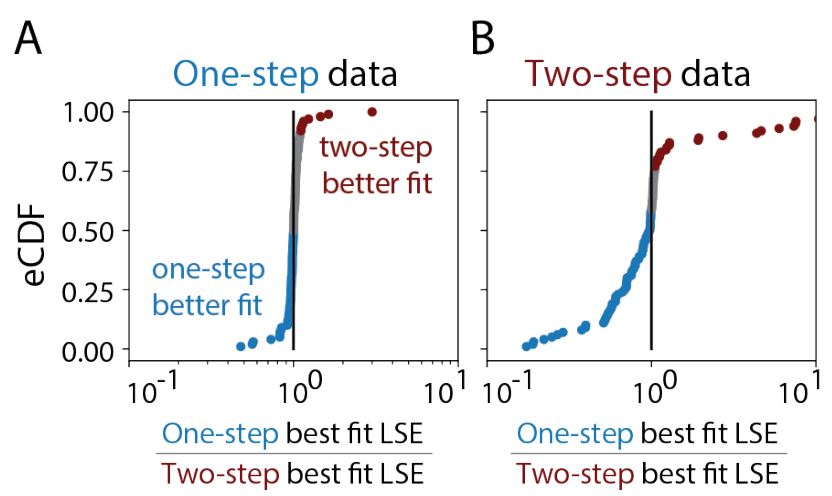

Figure 3.9: One-step model provides better fits of most simulated data, whether generated from one-step or two-step model. In the absence of an analytical likelihood function to perform model selection, we approximated the relative likelihood of the two models with the ratio of the least squared error of the best one-step and two-step fits of the same data. The one-step model is considered a better fit when $\frac{\text { One-step best fit LSE }}{\text { Two-step best fit LSE }} \leq 1$, and the twostep model is considered a better fit when $\frac{\text { One-step best fit LSE }}{\text { Two-step best fit LSE }}>1.5$. When $1<\frac{\text { One-step best fit LSE }}{\text { Two-step best fit LSE }} \leq 1.5$, the more likely model is ambiguous, as the two-step model provides only a small improvement in fit but is more complex. Blue, red, and gray dots show these three cases, respectively. Data generated from a one-step model is fit better by a one-step model for roughly $50 \%$ of simulations, and a further $45 \%$ are ambiguous. By contrast, data generated from a two-step model is better fit by a one-step model or is ambiugous in $75 \%$ of cases.

\subsubsection{Ligand-specific differences in affinity and receptor-specific differences in activity may be measured directly.}

We hypothesize that the diverse patterns of combinatorial and context-dependent responses to BMP emerge from specific configurations of receptor affinities and activities, which we estimated by fitting a model of BMP signaling to the data. While we spent significant effort estimating these parameters indirectly (Sections 2.3.8 and 3.2.1), the biochemical parameters could be estimated more directly with alternative experimental methods. Though the number of receptor-ligand interactions is perhaps too large to characterize exhaustively, these measurements could focus on either poorly-constrained parameter or certain features that were consistent across all top fits. For example, we predict that BMP10 has higher affinity for ACVR1+BMPR2 than BMP7 and BMP9 (Figure 3.2E), and that ACVR1-containing complexes are higher activity than BMPR1A-containing complexes (Figures 3.2 I-N).

The most well-established method for measuring ligand-receptor affinity is surface plasmon resonance (SPR) (see published data summarized in Figure 3.7). However, these measurements are challenging to interpret, as the multivalency of binding at the cell surface may produce a different effective affinity than measured by SPR, and the affinities estimated from "equilibrium" and "kinetic" measurements can vary substantially. Using single-particle tracking to observe the dynamics 
of ligands and receptors interacting may provide a useful alternative for determining some of these affinities in live cells.

Measuring activities of individual signaling complexes presents a more significant challenge. While the differential activity of different signaling complexes can be inferred by comparing pathway activation by the same ligand in different cells expressing different receptors,,$\sqrt[30[42]{ } \text { ideally }$ the activity of different complexes could be compared in the same cell. Many small molecule inhibitors of ACVR1, an important drug target for Fibrodysplasia Ossificans Progressiva (FOP) and Diffuse Intrinsic Pontine Glioma (DIPG), have been developed.13/38|39|53|14|65] Of these, a few show promising selectivity for ACVR1 over BMPR1A, the other Type I receptor expressed in most of our cell lines. Saracatinib has $10 \mathrm{x}$ lower $I C_{50}$ against BMPR1A relative to ACVR1 when inhibiting constitutively active receptors expressed in $\mathrm{C} 2 \mathrm{C} 12$ cells. ${ }^{65} \mathrm{LDN}-212854$, in a similar assay, also inhibited ACVR1 at 10x lower concentrations than any other receptor. $\frac{38}{2}$ Two other molecules showed 33x and 300x selectivity against ACVR1 over BMPR1A (M4K2009 and ML347, respectively) in an assay where receptor kinase activity is examined in a solution of ATP,$\sqrt[53 \mid 13]{13}$ though this assay may overestimate the actual selectivity in real cells. 65

Assuming one of these four molecules is sufficiently selective for ACVR1 over BMPR1A, the kinase activity of ACVR1 can be inhibited in cells where both receptors are active. If, as the model predicts, the minority of ACVR1-containing complexes produce the majority of the output, inhibition of ACVR1 in NMuMG will remove most ligands' ability to activate, even though BMPR1A expression is five-fold higher (Figure 2.4A). It is particularly striking to consider if chemically inhibiting ACVR1 produces a different effect than knocking the receptor out. We hypothesize that in the ACVR1 knockout, ligands bind more BMPR1A than when ACVR1 is present, so that the ACVR1 knockdown represents not only the removal of ACVR1-containing complexes but also the rearrangement of other signaling complexes. In the absence of a chemical inhibitor, however, replacing ACVR1 with a kinase-inactive version could also reveal how much output is produced from ACVR1-containing complexes formed in the presence of BMPR1A. 56

\subsection{Connecting simple ligand pairs and single receptor perturbations to the larger world of BMP signaling}

While this thesis purports to explore combinatorial and context-dependent BMP signaling, the work presented here takes a fairly narrow view of combinations and context. In real world settings where BMP guides skeletal formation or healing, the BMP pathway takes in a wide array of inputs, including combinations of more than two BMPs (Section 3.3.1), BMP heterodimers (Section 3.3.2), and non-BMP ligands that activate Smad1/5/8 or share receptors with BMP (Section 3.3.3). Moreover, tissue contexts differ in more than BMP receptor expression (Section 3.3.4), and receptor- 
ligand interactions govern not only the phosporylation of Smad1/5/8, the output we quantified in our pairwise analysis, but also other outputs such as cell fate (Section 3.3.5). In the following sections, I outline future experiments that can connect the findings here to the more complex realities of BMP signaling as it occurs in organismal contexts.

\subsubsection{Responses to combinations of more than two ligands can have non-trivial differences from pairwise responses.}

While we primarily measured responses to pairs of BMPs, naturally occurring biological systems include combinations of more than two ligands. A general study of developing mice showed BMP2, BMP4, and BMP7 were often coexpressed in many organs. ${ }^{10}$ In the developing skeleton, BMP2, BMP4, BMP5, and BMP7 can be expressed in overlapping regions. ${ }^{633}$ Similarly, BMP4, BMP5, and BMP7 are all expressed from the roof plate in the developing chick brain,, 31 and BMP2, BMP4, BMP5, BMP6, BMP7, BMP9, and GDF7 are all detected at various points in spinal cord development. 12 An equally large number of ligands are expressed during kidney development, though their spatial overlap may be somewhat limited. ${ }^{52}$ Therefore, a more complete description of BMP combinations would describe their behavior in combinations of more than two.

When predicting responses to arbitrary combinations of BMP ligands, we would prefer to measure only a subset of all possible combinations, and then fit a mathematical model that describes the response to any combination. A model with full mechanistic detail can, by virtue of capturing all interactions and effects, compute the response to an arbitrary number of inputs. While the BMP model used in this paper (Section 2.3.8) abstracts away certain details about signaling, it summarizes the majority of ligand-receptor interactions and therefore could be used to predict combinatorial responses. However, this requires knowing all the model's fit parameters, which have not yet been determined. A second alternative is a mechanism-free model that describes emergent higher-order interactions using effective parameters to summarize a large set of unknown interactions. These models have been particularly useful in describing drug and gene interactions, where it is difficult to model all pleiotropic effects of individual drugs or genes on large-scale cellular processes like DNA replication or protein translation.

When parameterizing higher order interactions, many studies of antibiotics define three-way interactions in analogy to pairwise interactions.66[58/45 Similar to how an additive expectation for individual drug effects can identify surprising "non-additivity" between pairs, "non-additive" pairwise interactions have an expected additive effect on the three-way interaction. However, these "additive" models still have large errors even in their most predictive formulation,, 45 and can vary considerably depending on which kind of behavior is classified as additive.

Nonetheless, many other mathematical expressions can describe how pairwise interactions col- 
lectively contribute to a higher-order interaction, even if these expressions are not derived from some intuition about how additivity should occur. One example of this is the "dose model," where the presence of additional drugs causes each drug to have an effect at a different "effective" concentration $\left[\frac{6845}{}\right.$ In synergistic interactions, a drug is at effectively higher concentrations, while in antagonistic interactions, drugs are effectively at lower concentrations. Such a model predicted responses to combinations of up to 10 drugs with higher accuracy than the most predictive deviation from additivity model .2845$]$ While the dose model seems the most promising candidate for predicting responses to combinations of BMPs, the expressions are currently framed in terms of antibiotic effects and should be adapted for signaling proteins. Here, I first summarize the dose model as implemented for antibiotics and then describe an alternative framework for signaling proteins.

The model is deployed in three steps. First, individual drug behavior is parameterized with a Hill function, showing how growth rates decay from 1 to 0 when additional drug is added:

$$
g_{i}\left(D_{i}\right)=\frac{1}{1+\left(D_{i} / D_{i}^{50}\right)^{n_{i}}}
$$

where $g_{i}$ is the microbial growth rate (normalized between 1 and 0 ), $D_{i}$ is the concentration of the drug, $D_{i}^{50}$ is the concentration at which the drug has half of its maximal effect, and $n_{i}$ is the Hill coefficient. The $i$ index indicates that each drug has a unique Hill function.

Second, pairwise behavior is parameterized with the following expressions:

$$
\begin{aligned}
g_{i j}\left(D_{i}, D_{j}\right) & =g_{i}\left(D_{i}^{e f f}\right) g_{j}\left(D_{j}^{e f f}\right) \\
D_{i}^{e f f} & =D_{i}\left[1+a_{i j} \frac{D_{j}^{e f f} / D_{j}^{50}}{1+\left(D_{j}^{e f f} / D_{j}^{50}\right)}\right]^{-1} \\
D_{j}^{e f f} & =D_{j}\left[1+a_{j i} \frac{D_{i}^{e f f} / D_{i}^{50}}{1+\left(D_{i}^{e f f} / D_{i}^{50}\right)}\right]^{-1}
\end{aligned}
$$

where $g_{i j}\left(D_{i}, D_{j}\right)$ is the normalized growth rate in the presence of drugs $i$ and $j$ at the indicated doses. $D_{i}^{e f f}$ and $D_{j}^{e f f}$ are the effective concentrations of these drugs, due to their mutual influence summarized in $a_{i j}$ (the effect of drug $j$ on drug $i$ ) and $a_{j i}$ (the effect of drug $i$ on drug $j$ ). Note that each pair is parameterized with two fit parameters $\left(a_{i j}\right.$ and $\left.a_{j i}\right)$, which are not symmetric, and have a lower bound of -1 . For a given combination of $D_{i}$ and $D_{j}$, Equations 3.3 and 3.4 are solved 
simultaneously to find $D_{i}^{e f f}$ and $D_{j}^{e f f}$, which are plugged into Equation 3.1 to then compute the overall effect of $g_{i}$ and $g_{j}$ on $g_{i j} . a_{i j}$ and $a_{j i}$ are fit parameters that minimize the error between the predicted $g_{i j}\left(D_{i}, D_{j} \mid a_{i j}, a_{j i}\right)$ and the measured growth rate in the presence of the drug pair.

To finally predict the effect of a combination of up to $N$ drugs, the full set of pairwise parameters $a_{i j}$ and $a_{j i}$ are used to determine the effective dose of each drug.

$$
\begin{aligned}
g_{1, \ldots, N}\left(D_{1}, \ldots, D_{N}\right) & =\prod_{i=1}^{N} g_{i}\left(D_{i}^{e f f}\right) \\
D_{i}^{e f f} & =D_{i} \prod_{j \neq i}\left[1+a_{i j} \frac{\frac{D_{j}^{e f f}}{D_{j}^{50}}}{1+\frac{D_{j}^{e f f}}{D_{j}^{50}}}\right]^{-1}
\end{aligned}
$$

In a similar procedure as above, the set of $N D_{i}^{e f f}$ expressions are solved simultaneously to find the effective doses, which are then plugged into their respective Hill functions, whose overall product is the predicted growth rate in the presence of all drugs.

Adapting this approach for ligands requires a few crucial changes. First, ligands activate the pathway, whereas drugs decrease growth rates. Also, whereas all drugs have the same maximal effect of decreasing growth rate to zero, individual ligands may saturate at different levels of pathway activation (e.g. Figure S2.1D). Therefore, ligand activation $\left(S_{i}\right)$ should be expressed as:

$$
S_{i}=\rho_{i} \frac{\left(D_{i} / D_{i}^{50}\right)^{n_{i}}}{1+\left(D_{i} / D_{i}^{50}\right)^{n_{i}}}
$$

where $\rho_{i}$ captures the ligand-specific maximal effect. We further update the expressions for $g_{i j}$ and $g_{1, \ldots, N}$ as:

$$
\begin{aligned}
1-S_{i j} & =\left[1-S_{i}\left(D_{i}^{e f f}\right)\right]\left[1-S_{j}\left(D_{j}^{e f f}\right)\right] \\
\left(1-S_{1, \ldots, N}\right) & =\prod_{i=1}^{N} 1-S_{i}
\end{aligned}
$$

The prediction that the product of individual effects can model the combinatorial effect arises from antibiotic models of additivity that assume statistical independence of the different drugs. 
Therefore, instead of the product model, we may consider an additive model, as an analogy to the way the BMP model sums over different $T_{i j k}$ signaling complexes to compute the overall activation level:

$$
\begin{aligned}
S_{i j} & =S_{i}\left(D_{i}^{e f f}\right)+S_{j}\left(D_{j}^{e f f}\right) \\
S_{1, \ldots, N} & =\sum_{i=1}^{N} S_{i}\left(D_{i}^{e f f}\right)
\end{aligned}
$$

Adapting this "dose" model for predicting BMP interactions has dual usefulness. While a mechanistic model is more interpretable and elegant than a non-mechanistic model, a non-mechanistic model can provide faster and equally accurate predictions for higher order interactions. Moreover, if higher-order interactions could be predicted from pairwise responses alone, existing pairwise datasets (as in Chapter 2) may contain full information for predicting responses to combinations of three or more BMPs, even while not fully constraining our mechanistic model. Alternatively, if responses to combinations of more than two ligands cannot be predicted from the pairwise responses, this may provide some evidence for "higher-order" interactions, the origin of which can be determined by examining the full mechanistic model. Lastly, in addition to the dose model, mathematical frameworks developed for other systems with higher order interactions could be explored, in addition to the dose model. These includes models for microbial interactions, $\frac{1663557}{15}$ gene epistasis, ${ }^{1964}$ transcription factor synergy, ${ }^{15 / 44}$ and neuron correlation. .50

\subsubsection{BMP heterodimers and chimeras represent novel competitors for BMP receptors.}

The context-dependent, combinatorial interactions between BMP ligands emerge from affinitymediated competition for BMP receptors. So far, our work as has focused on BMP homodimers, where each dimeric subunit is the protein product of the same BMP gene. However, a cell expressing two different BMP genes can secrete both homodimers and heterodimers of the two genes. .27 In an impressive diversity of in vivo and in vitro assays across rat, zebrafish, and Xenopus, BMP heterodimers (especially BMP2/6 and BMP2/7) induced higher gene expression or bone formation than the same mass of homodimers. $227|55| 54|62| 40|37|$ Thus, heterodimers are largely considered to be more "potent" than homodimers. The distinct effects of heterodimers also have consequences in developmental processes, such as the role for BMP6/GDF7 heterodimers in axon repulsion. ${ }^{8}$ Removing BMP9/BMP10 heterodimers from plasma reduces its ability to activate the BMP pathway, $\underset{60}{6}$ and a BMP7 mutant that forms non-functional heterodimers is more lethal than a BMP7 knockout in mice. ${ }^{29}$ 
Heterodimers differ from homodimers in two principle ways. First, they have novel Type I receptor binding domains, as the portion of the ligand which binds the Type I receptor contacts both dimeric subunits. ${ }^{41}$ The Type II receptor binding domains are unchanged from the homodimers. Second, heterodimers have four different receptor binding domains, whereas homodimers have two repeats of two receptor binding domains. These physical differences from the basis for various predictions about the unique properties of heterodimers relative to homodimers. The majority resemble arguments to explain differences between BMP homodimers, as heterodimers can be thought of as a new point in ligand affinity and activity space. Heterodimers likely have different affinities for inhibitory ligand binding molecules (such as Noggin), as well as unique affinities for Type I receptors. They also represent unique combinations of affinities. As noted above BMP2 and BMP7 have higher affinity for Type I and Type II receptors, respectively (Figure 3.7). Therefore, a BMP2/7 heterodimer represents a unique combination of high affinity for both Type I and Type II receptors that has not yet been observed in any BMP homodimer. However, an additional possibility is that the collocalization of four unique BMP receptors around the heterodimeric ligand produces a novel, poorly understood interaction that has higher potency.

Much of the data collected so far suggests that differences in affinity alone are sufficient to explain the higher activity of heterodimers, by mass, than similar molecular weight homodimers. At endogenous concentrations, BMP2/7 alone produces sufficiently high pathway activation to pattern the dorsoventral axis of a zebrafish embryo, whereas BMP2 and BMP7 cannot. ${ }^{32}$ However, overexpression of BMP2, even in the absence of BMP7, can produce even higher than wildtype levels of pathway activation, $\frac{56}{5}$ suggesting that homodimers do not produce qualitatively different pathway activation but can, with higher concentrations, achieve the same output through their preferred Type I receptors. While BMP2/7 heterodimers do assemble zebrafish homologs of BMPR1A and ACVR1 in the same signaling complex, which BMP2 or BMP7 homodimers alone cannot, ${ }^{32}$ it's not clear that the combination of BMPR1A and ACVR1 in a single complex can produce a different outcome than complexes containing BMPR1A or ACVR1 alone. Even more convincingly, engineering BMP ligand chimeras with novel combinations of receptor binding domains also increases their potency $\frac{51}{1}$ (Intriguingly, follow-up work on BMP signaling in zebrafish embryos suggests that even kinase-dead BMPR1A that cannot activate the pathway can rescue a BMPR1A knockout, consistent with our earlier prediction that ACVR1-containing complexes produce the majority of pathway activation, cf. Figure S2.8 $\mathrm{B}$.)

The unique ligand-receptor interactions of BMP heterodimers and chimeras (whether qualitatively or quantitatively distinct) can extend our understanding of how equivalence emerges from affinity and activity. For example, heterodimers are expected to cluster with neither of their related homodimers, as they have vastly different affinities. However, the magnitude of this effect may 
depend on the availability of the high affinity receptors that the heterodimer preferentially signals through. As with other ligands, heterodimers may more closely resemble homodimers in certain receptor contexts, producing a unique pattern of context-dependent equivalence. Lastly, as heterodimers have four unique binding interfaces, they may simultaneously have low and high affinity for the same receptor, depending on which interface is being considered. Our current model, which considers only one affinity of each ligand for each Type I-Type II receptor pair, would require additional parameters to capture this behavior. Further experiments in our in vitro system may also distinguish between the hypotheses that heterodimers assemble the same signaling complexes with higher affinity or that they assemble unique tetramers of receptors. If heterodimers produce qualitatively distinct outputs, then no amount of BMP2 or BMP7 should achieve the same level of pathway activation. Comparing dose responses of BMP2/7 with BMP2, BMP7, or an equimolar mix of BMP2 and BMP7 could distinguish these two cases.

\subsubsection{BMPs may combine in unique ways with other molecules that share a subset of BMP receptors.}

The BMP ligands are part of a larger TGF- $\beta$ superfamily of ligands that have similar structures and bind similar receptors. Canonically, these ligands can activate either Smad1/5/8 or Smad2/3, and their specification depends on whether they bind Type I receptors that activate Smad1/5/8 or Smad2/3. They nonetheless share Type II receptors, such as BMPR2. Smad2/3 ligands, such as Activin A, which bind Type II receptors more strongly than others ligands, can antagonize Smad1/5/8 ligands that require the same Type II receptor to signal ${ }^{[3}$ However, recent evidence shows that TGF- $\beta$ may form a variety of complexes with BMPR2, activating both Smad2/3 and Smad1/5/8. ${ }^{23}$ Moreover, Activin A may also activate ACVR1 in myeloma cells, ${ }^{43}$ although other work showed Activin A produced non-signaling complexes when bound to ACVR1 in HEK293 cells. ${ }^{4}$ Lastly, some BMP ligands may also promiscuously activate Smad2/3. A large screen of cancer cell lines found that BMP2 could activate Smad2/3 in some lines, ${ }^{25}$ although receptor mutations, such as the FOP ACVR1 R206H mutation that confers Smad1/5/8 activity to Activin

$\mathrm{A},{ }^{4}$ may explain some of these observations. While this collection of literature is inconsistent and teems with minutia, it supports the overall point that receptor-ligand interactions are likely broader than we suppose, and future models of BMP signaling should consider the potential functionality of these shared receptors regulating the different Smad outputs. 


\subsubsection{Perturbations of other pathway components can produce a new cell context for BMP signaling.}

Responses to BMP proteins vary between cell context, and we largely tried to explain the tissuespecific responses by considering differences in BMP receptor expression alone. As BMP ligands directly bind and interact with the receptors, receptors are one of the strongest determinants of cellular responsiveness. However, many other proteins also facilitate or direct pathway activation and may be responsible for some of the context-dependent effects observed in vivo. These act at three different levels: signaling complex formation, Smad activation, and transcriptional regulation (Figure 3.10). I review them here as a source of alternative hypotheses for explaining contextdependence in vivo and as an inspiration for future experiments.

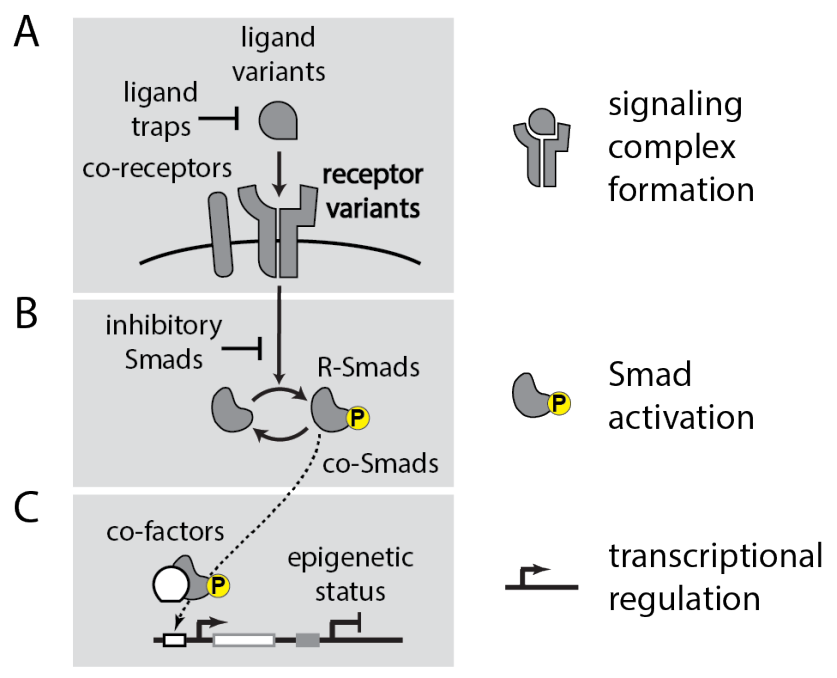

Figure 3.10: Signaling complex formation, Smad activation, and downstream transciptional outputs vary between BMP signaling contexts. The activation of target genes by extracellular BMPs depends on the activity of a variety of molecules. Broadly, they exert their influence at three levels.

(A) First, different BMP signals are conveyed by the use of ligand variants, receptor variants, ligand traps, and co-receptors that influence complex formation.

(B) Second, the abundance of inhibitory Smads, Smad4 (the co-Smad), and the relative amounts of Smad1, 5, and 8 influence which Smads are phosphorylated by BMP signaling complexes.

(C) Third, the pool of activated Smad is converted into gene expression, as co-factors complex with Smad to direct the complex to different target genes, which may or may not lie in accessible chromatin.

Though a full signaling complex requires only one ligand, one Type I receptor, and one Type II receptor, other molecules can inhibit or promote the formation of these complexes. Ligandspecific co-receptors, suc as endoglin, MuSK, and Repulsive Guidance Molecules, can facilitate the formation of signaling complexes. .5967 The presence of ligand traps or other inhibitors, such 
as Gremlin, Noggin, and Chordin, may also make one tissue context more amenable to BMP signaling than another. Lastly, the behavior of ligand and receptor variants can be made even more diverse by post-translational modifications, ${ }^{21}$ which may manifest in context-specific expression of the enzymes that effect these modifications. Together, these molecules influence which signaling complexes form and in what abundance (Figure 3.10A).

Once the signaling complexes have formed, phosphorylated Type I receptors can activate downstream R-Smads, specifically Smad1/5/8 in the case of BMP ligands (Figure 3.10B). In order to activate gene expression, Smads must form trimeric complexes, most often with Smad4, the coSmad ${ }^{49}$ However, the same ligand-receptor signaling complexes can produce different amounts of activated Smad if there is excess inhibitory Smad or limited co-Smad. ${ }^{24 \mid 11}$ Moreover, the identity of the Smad molecules in these complexes appears to influence which genes are upregulated by BMP pathway activation. ${ }^{34}$ Post-translational modifications of Smad2/3 (and likely Smad1/5/8) can also produce distinct Smad molecules that convey different messages, such as mitogenic, fibrogenic, or migratory signals. $\frac{36}{3}$ Therefore, different expression levels of genes controlling these processes, such as total Smad levels or the presence and absence of certain kinases, could also produce context-dependent effects, by redirecting signaling complex formation into different downstream outputs.

Once active, Smad trimers translocate to the cell nucleus, where they can activate gene expression (Figure 3.10C). However, Smads have relatively low affinity for DNA and bind to a broadly defined consensus sequence. 1124 Therefore, Smads are directed to gene promoters by co-factors, which provide increased specificity and affinity. ${ }^{35 \mid 11}$ Nonetheless, whether Smad complexes have access to a given locus depends on chromatin accessibility, which may also direct the same BMP inputs into the expression of different target gene programs. BMP activation can also affect chromatin accessibility as Smads interact with histone modifying enzymes. 11

Overall, this careful accounting of all the molecules involved in BMP pathway activation highlights the many possible causes of context-dependent responses observed in development. Though we attribute some of them to differences in receptor expression, these other factors likely play a role in certain processes. This therefore expands the list of possible differentially expressed genes to consider when identifying which molecules determine BMP responsiveness. Future cell line engineering could also focus on systematically perturbing some of the molecules described here, to determine how they redirect BMP signaling into different downstream effects. 


\subsubsection{Receptor-ligand computations govern other immediate outputs and downstream cell fates.}

The work presented here largely focuses on activation of canonical Smad1/5/8 transcription factors, but these same BMP ligands activate both alternative immediate outputs as well as diverse downstream outputs. These include immediate activation of Smad2/3 (cf. Section ??) or noncanonical outputs, ${ }^{48 / 2017}$ as well as distinct target genes. Together, these changes can alter cell fate, the effect which led to the discovery of BMP signaling ${ }^{61}$ and which drives current interest in BMP therapies. ${ }^{46}$ As the "M" in BMP indicates, BMPs can signal as morphogens, where distinct concentrations of protein generate different fates. ${ }^{18}$ Knowing the amount of pathway activation produced by individual ligands and pairs could allow the rational production of certain cell fates, using either individual BMPs or combinations to produce the desired level of R-Smad activation.

By contrast, at least one other report has claimed that different BMPs may give rise to unique cell fates. ${ }^{1}$ In these experiments, embryoid bodies derived from mouse embryonic stem cells (mESCs) were co-cultured in retinoic acid and one BMP for nine days. Surprisingly, different BMPs induced expression of different target genes, corresponding to different dorsal interneuron fates. BMP6 and GDF7 mostly initiated expression of a roof plate (RP) fate marker, and small amounts of a dorsal interneuron 1 (dI1) fate marker. BMP4 mostly induced expression of the dI1 fate marker and some RP. BMP5 and BMP7 did not induce appreciable expression of any of these target genes. When given in pairs, each of the BMPs largely inhibited the other's induction of dI1 or RP target genes. However, when all BMPs were given simultaneously, the RP target gene was highly expressed.

Given our predictions of equivalence of BMP signals in an mESC context, these results are surprising for two reasons. First, we might expect that the equivalence groups we observed in mESCs (Figure 2.4) also capture intrinsic differences in the effects of these ligands on fates. However, the data from [1] shows that BMP6 produces very different effects on gene expression than BMP5 and BMP7, despite being in the same equivalence group in our study of mESCs (Figure 2.4E). Also, while BMP6 and GDF7 are expected to be in different equivalence groups, they produce the same effects on target gene expression. The pairwise interactions between these groups are also unexpected, as BMP4 can antagonise BMP6's induction of the roof plate target gene, while BMP6 can antagonize BMP4's induction of the dI1 gene. By contrast, BMP4 and BMP6 combined additively in the mESCs we studied (Figure 2.4D). Thus, these results present an alternative map for "functional" BMP equivalance groups, with respect to cell fates rather than activation of Smad1/5/8.

The disagreement between the apparent equivalence of ligands in these supposedly similar cell lines may occur for many reasons. First, the mESCs undergo several transformations, first forming embryoid bodies and then being cultured for a day in retinoic acid alone. This provides time 
for a large change in BMP receptor expression or some other BMP pathway machinery. Second, the mESCs used for the two experiments were procured from different sources. and may start the experiment with different receptor expression profiles. Third, the ligands may activate other outputs besides Smad1/5/8 that are not measured in our experiments, such that we do not detect the pertinent ligand difference governing stem cell fates. To distinguish between these two hypotheses, we could grow embryoid bodies from our own mESCs, with known equivalence groups. Culturing them with different BMPs from the various equivalence groups, individually and in pairs, can reveal if a ligand's effect on cell fate correlates with its equivalence group, and if the mutual effect of two ligands on a target gene matches the expected pairwise interaction. If not, qPCR can determine if the differentiation protocol changed BMP receptor expression, and therefore the equivalence map, or if the ligand-specific effects arise independently of the similarity of their effects on pSmad1/5/8. 


\subsection{Supplementary Figures}

A

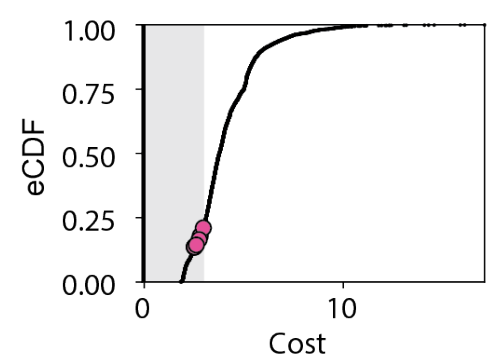

B

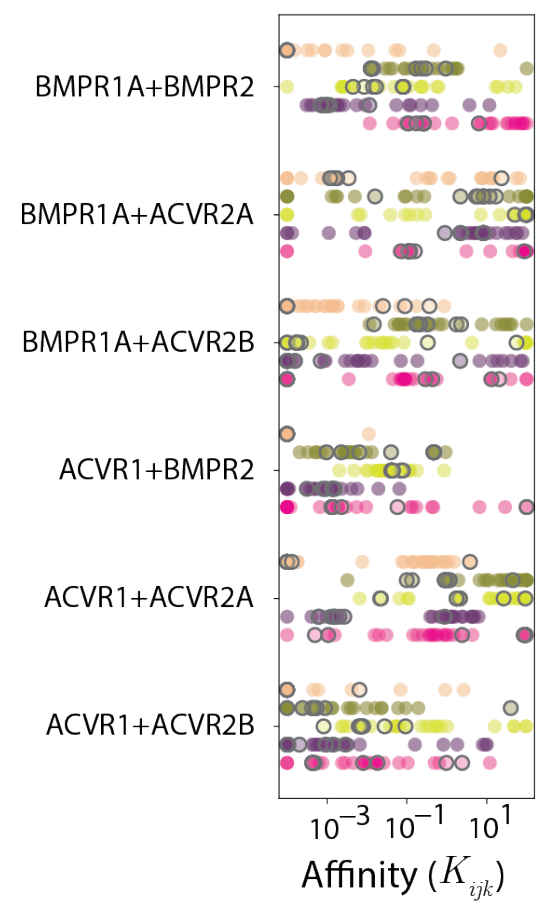

- Selected new fits
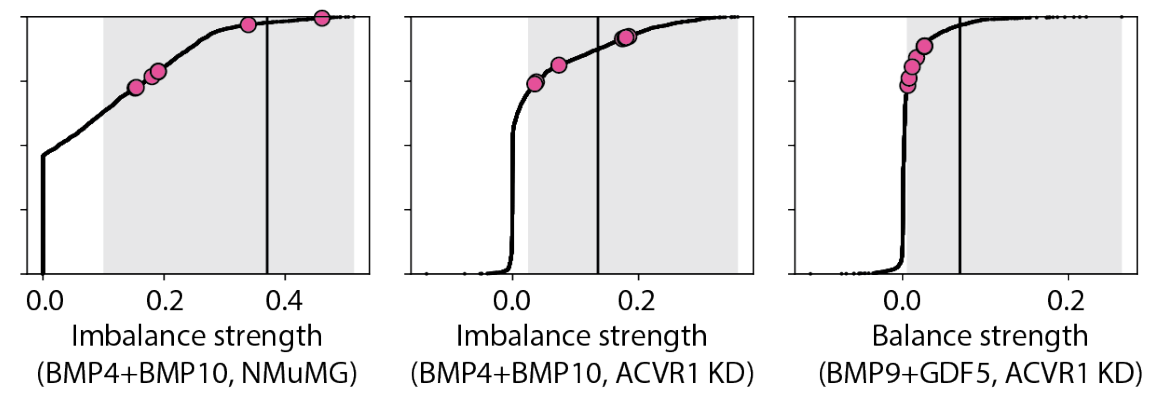

C

Dissimilarity of best fit parameters

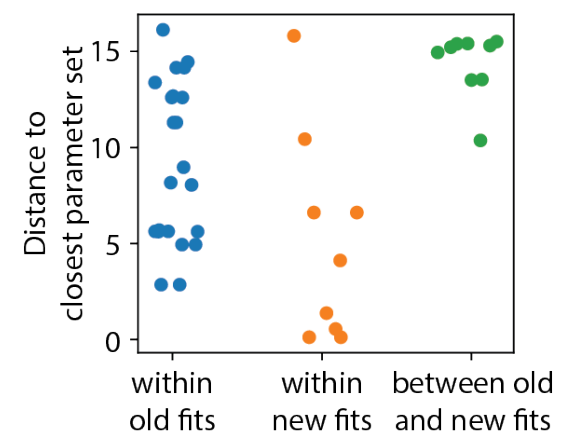

Figure S3.1: Parameters estimated by fitting either pairwise data and dose responses or pairwise data alone are qualitatively similar. (caption on following page) 
Figure S3.1 (previous page):

(A) Parameters were estimated with 6000 gradient descent runs to minimize least squared error between the model and the data. Each gradient descent run produced a unique solution. A top 9 fits were selected using the criteria shown here. Each subplot shows a different metric: cost (or overall least squared error), the strength of the BMP4+BMP10 imbalance in NMuMG, the strength of the BMP4+BMP10 imbalance in ACVR1 knockdown, and the strength of the BMP9+GDF5 balance in ACVR1 knockdown. Within each subplot, the black curve is the eCDF of that metric's strength in the 6000 fits, and the gray region shows the accepted metric strength for a "top fit." The gray region also contains the vertical black line indicating the observed strength of this metric in the data. The pink dots correspond to the 9 parameter fitting results that satisfy all four criteria simultaneously. (B) Plotting the values for the top fits for both the "old" and "new" parameter fitting approaches shows that the "new" fits are largely a subset of the "old" fits. The two subplots show the affinities and activities of each signaling complex, grouped by unique Type I and Type II receptor pairs (ytick labels) and colored by the ligands. The colored dots indicate the previous parameter estimates (Section 2.3.8), and the black outlines correspond to the updated estimates (Section 3.2.1). These black outlines overlap with some region of the estimates from the previous run. However, given that the new fits do produce better fits of the gradient dose responses (Figure 3.1B), they may represent unique combinations or differently-tuned values.

(C) To quantify dissimilarity between the "old" and "new" parameter estimates, we computed each parameter set's minimum Euclidean distance to another parameter set, as a measure of how far it was from it's nearest neighbor. Within the set of only "old" or "new" fits, the new fits were closer together. However, each "new" fit was at least as close to one of the "old" fits as the "old" fits are to each other. This indicates that these new results do not have large quantitative differences from the previously-generated results. 
A

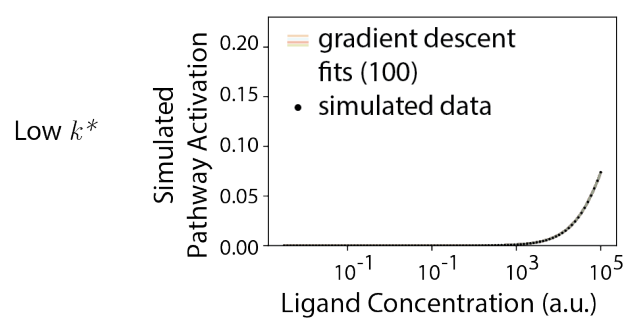

$c$

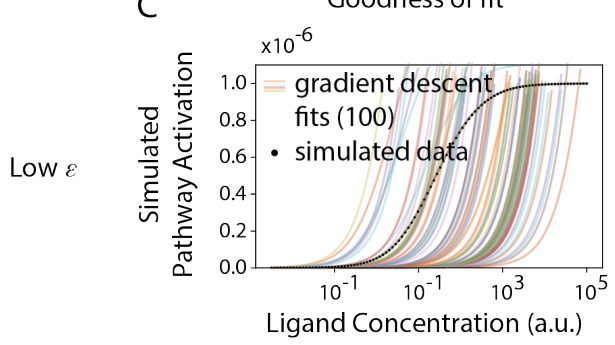

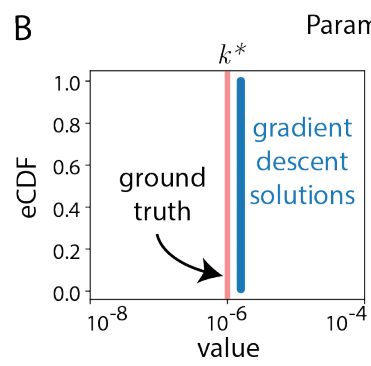

Parameter estimates (by gradient descent)
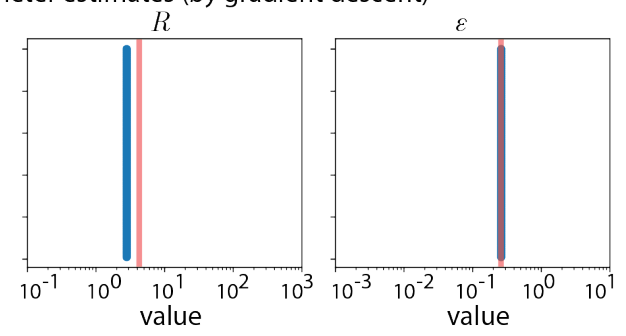

D

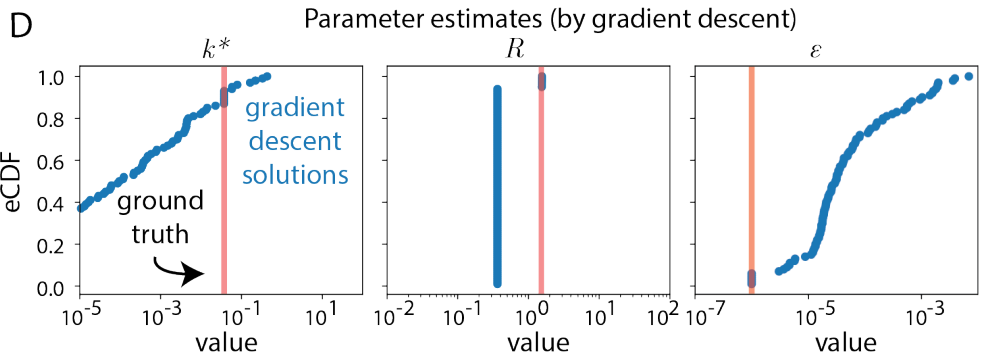

Figure S3.2: Very low affinity values harder to estimate than very low activity values. Data simulated from parameter sets containing a very low affinity $\left(k^{*}\right)$ or activity $(\epsilon)$ were more challenging to fit, as shown with these two examples of random parameter sets.

(A) Noisy data (dots) were simulated for a random parameter set where $k^{*}=10^{-6}$. Data were simulated over a fixed ligand concentration range, which did not include the linear or saturating regimes of the dose response. Many parameter estimates (i.e. the 100 gradient descent fits shown in B) produce dose responses consistent with these data.

(B) eCDFs of 100 estimates for each parameter are shown in blue, relative to the true parameter values indicated with the vertical red line. Nearly every estimate includes the same overestimate of $k^{*}$ and an overestimate of $R$.

(C) Noisy data (dots) were simulated for a random parameter set where $\epsilon=10^{-6}$. Because the signaling complex is essentially off, the pathway response does not reach a very high value. Widely-varying parameter estimates, trading off a higher activity value with a lower affinity, produce overlapping fits with small absolute errors.

(D) Despite the wide variety of parameter values that fit the data with low error, some parameter estimates overlap with the true value. The true value, with close to zero error, can thus be selected from among the many gradient descent solutions.

See also Figure 3.4

\section{References}

[1] Madeline G Andrews et al. "BMPs direct sensory interneuron identity in the developing spinal cord using signal-specific not morphogenic activities”. en. In: Elife 6 (Sept. 2017). ISSN: 2050-084X. DOI: $10.7554 /$ eLife. 30647. 
[2] Aki Aono et al. "Potent ectopic bone-inducing activity of bone morphogenetic protein4/7 heterodimer". In: Biochemical and biophysical research communications 210.3 (1995), pp. 670-677.

[3] Senem Aykul and Erik Martinez-Hackert. "Transforming Growth Factor- $\beta$ Family Ligands Can Function as Antagonists by Competing for Type II Receptor Binding”. en. In: J. Biol. Chem. 291.20 (May 2016), pp. 10792-10804. ISSN: 0021-9258, 1083-351X. DOI: 10.1074/ jbc.M115.713487

[4] Senem Aykul et al. "Activin A forms a non-signaling complex with ACVR1 and type II Activin/BMP receptors via its finger 2 tip loop”. en. In: Elife 9 (June 2020). ISSN: 2050084X. DOI: $10.7554 /$ eLife. 54582 .

[5] Jodie L Babitt et al. "Repulsive guidance molecule (RGMa), a DRAGON homologue, is a bone morphogenetic protein co-receptor". en. In: J. Biol. Chem. 280.33 (Aug. 2005), pp. 29820-29827. ISSN: 0021-9258. DOI: $10.1074 /$ jbc . M503511200.

[6] Amitabha Bandyopadhyay et al. "Genetic analysis of the roles of BMP2, BMP4, and BMP7 in limb patterning and skeletogenesis". en. In: PLoS Genet. 2.12 (Dec. 2006), e216. ISSN: 1553-7390, 1553-7404. DOI: 10.1371/journal.pgen.0020216.

[7] Ira L Blitz and Ken W Y Cho. "Finding partners: how BMPs select their targets". en. In: Dev. Dyn. 238.6 (June 2009), pp. 1321-1331. ISSN: 1058-8388. DoI: 10. 1002/dvdy . 21984.

[8] Samantha J Butler and Jane Dodd. "A role for BMP heterodimers in roof plate-mediated repulsion of commissural axons". en. In: Neuron 38.3 (May 2003), pp. 389-401. ISSN: 0896-6273. DOI: $10.1016 / \mathrm{s} 0896-6273(03) 00254-\mathrm{x}$

[9] S Cheifetz et al. "Endoglin is a component of the transforming growth factor-beta receptor system in human endothelial cells". en. In: J. Biol. Chem. 267.27 (Sept. 1992), pp. 1902719030. ISSN: 0021-9258.

[10] Shahab M Danesh et al. "BMP and BMP receptor expression during murine organogenesis". en. In: Gene Expr. Patterns 9.5 (June 2009), pp. 255-265. ISSN: 1567-133X. DOI: 10. 1016/ j.gep.2009.04.002.

[11] Rik Derynck and Erine H Budi. "Specificity, versatility, and control of TGF- $\beta$ family signaling”. In: Science signaling 12.570 (2019).

[12] Gwenvael Le Dréau and Elisa Martí. “The multiple activities of BMPs during spinal cord development". In: Cellular and Molecular Life Sciences 70.22 (2013), pp. 4293-4305. ISSN: 1420-682X. DOI: 10.1007/s00018-013-1354-9.

[13] Darren W Engers et al. "Synthesis and structure-activity relationships of a novel and selective bone morphogenetic protein receptor (BMP) inhibitor derived from the pyrazolo [1.5-a] pyrimidine scaffold of Dorsomorphin: the discovery of ML347 as an ALK2 versus ALK3 selective MLPCN probe”. In: Bioorganic \& medicinal chemistry letters 23.11 (2013), pp. 3248-3252.

[14] Deeba Ensan et al. "Targeting ALK2: an open science approach to developing therapeutics for the treatment of diffuse intrinsic pontine glioma". In: Journal of medicinal chemistry 63.9 (2020), pp. 4978-4996. 
[15] Javier Estrada et al. "Information integration and energy expenditure in gene regulation". In: Cell 166.1 (2016), pp. 234-244.

[16] Karoline Faust and Jeroen Raes. "Microbial interactions: from networks to models". In: Nature Reviews Microbiology 10.8 (2012), pp. 538-550.

[17] Lilach Gilboa et al. "Bone morphogenetic protein receptor complexes on the surface of live cells: a new oligomerization mode for serine/threonine kinase receptors". In: Molecular biology of the cell 11.3 (2000), pp. 1023-1035.

[18] Hannah Greenfeld, Jerome Lin, and Mary C Mullins. "The BMP signaling gradient is interpreted through concentration thresholds in dorsal-ventral axial patterning". In: PLoS Biol. 19.1 (Jan. 2021), e3001059. ISSN: 1544-9173, 1545-7885. DoI: 10. 1371/ journal. pbio. 3001059.

[19] Rafael F Guerrero et al. "Proteostasis environment shapes higher-order epistasis operating on antibiotic resistance". In: Genetics 212.2 (2019), pp. 565-575.

[20] Lea M Gunnell et al. "TAK1 regulates cartilage and joint development via the MAPK and BMP signaling pathways". en. In: J. Bone Miner. Res. 25.8 (Aug. 2010), pp. 1784-1797. ISSN: 0884-0431, 1523-4681. DOI: $10.1002 / \mathrm{jbmr} .79$.

[21] Akiko Hata and Ye-Guang Chen. "TGF- $\beta$ signaling from receptors to Smads". In: Cold Spring Harbor perspectives in biology 8.9 (2016), a022061.

[22] Kai Heinecke et al. "Receptor oligomerization and beyond: a case study in bone morphogenetic proteins”. en. In: BMC Biol. 7 (Sept. 2009), p. 59. ISSN: 1741-7007. Dor: 10.1186/ 1741-7007-7-59.

[23] Christian Hiepen et al. "BMPR2 acts as a gatekeeper to protect endothelial cells from increased TGF $\beta$ responses and altered cell mechanics". en. In: PLoS Biol. 17.12 (Dec. 2019), e3000557. ISSN: 1544-9173, 1545-7885. DoI: 10.1371/journal .pbio.3000557.

[24] Caroline S Hill. "Transcriptional control by the SMADs". In: Cold Spring Harbor perspectives in biology 8.10 (2016), a022079.

[25] Alisha Holtzhausen et al. "Novel bone morphogenetic protein signaling through Smad2 and Smad3 to regulate cancer progression and development". In: The FASEB Journal 28.3 (2014), pp. 1248-1267.

[26] Michael J Isaacs et al. "Bone morphogenetic protein-2 and -6 heterodimer illustrates the nature of ligand-receptor assembly”. en. In: Mol. Endocrinol. 24.7 (July 2010), pp. 14691477. ISSN: 0888-8809, 1944-9917. DOI: 10.1210/me.2009-0496.

[27] David I Israel et al. "Heterodimeric bone morphogenetic proteins show enhanced activity in vitro and in vivo". In: Growth factors 13.3-4 (1996), pp. 291-300.

[28] Itay Katzir et al. "Prediction of ultra-high-order antibiotic combinations based on pairwise interactions". In: PLoS computational biology 15.1 (2019), e1006774.

[29] Hyung-Seok Kim et al. "BMP7 functions predominantly as a heterodimer with BMP2 or BMP4 during mammalian embryogenesis”. In: Elife 8 (2019), e48872. 
[30] Uwe Klammert et al. "GDF-5 can act as a context-dependent BMP-2 antagonist". en. In: BMC Biol. 13 (Sept. 2015), p. 77. ISSN: 1741-7007. DOI: 10.1186/s12915-015-0183-8.

[31] Karel F Liem, Gabi Tremml, and Thomas M Jessell. "A Role for the Roof Plate and Its Resident TGF $\beta$-Related Proteins in Neuronal Patterning in the Dorsal Spinal Cord". In: Cell 91.1 (1997), pp. 127-138. ISSN: 0092-8674. DOI: 10.1016/s0092-8674(01)80015-5.

[32] Shawn C Little and Mary C Mullins. "Bone morphogenetic protein heterodimers assemble heteromeric type I receptor complexes to pattern the dorsoventral axis". In: Nature cell biology 11.5 (2009), pp. 637-643.

[33] Carlos I Lorda-Diez et al. "Ligand- and Stage-Dependent Divergent Functions of BMP Signaling in the Differentiation of Embryonic Skeletogenic Progenitors In Vitro". In: Journal of Bone and Mineral Research 29.3 (2014), pp. 735-748. ISSN: 1523-4681. DOI: 10.1002/ jbmr. 2077 .

[34] Philippe Lucarelli et al. "Resolving the combinatorial complexity of smad protein complex formation and its link to gene expression". In: Cell systems 6.1 (2018), pp. 75-89.

[35] Joan Massagué. “TGF $\beta$ signalling in context”. In: Nature reviews Molecular cell biology 13.10 (2012), pp. 616-630.

[36] Koichi Matsuzaki. "Smad phospho-isoforms direct context-dependent TGF- $\beta$ signaling”. In: Cytokine \& growth factor reviews 24.4 (2013), pp. 385-399.

[37] Chunlei Miao et al. "BMP2/7 heterodimer enhances osteogenic differentiation of rat BMSCs via ERK signaling compared with respective homodimers". In: Journal of cellular biochemistry 120.5 (2019), pp. 8754-8763.

[38] Agustin H Mohedas et al. "Development of an ALK2-biased BMP type I receptor kinase inhibitor”. In: ACS chemical biology 8.6 (2013), pp. 1291-1302.

[39] Agustin H Mohedas et al. "Structure-activity relationship of 3, 5-diaryl-2-aminopyridine ALK2 inhibitors reveals unaltered binding affinity for fibrodysplasia ossificans progressiva causing mutants". In: Journal of medicinal chemistry 57.19 (2014), pp. 7900-7915.

[40] Tokimitsu Morimoto et al. "The bone morphogenetic protein-2/7 heterodimer is a stronger inducer of bone regeneration than the individual homodimers in a rat spinal fusion model". In: The Spine Journal 15.6 (2015), pp. 1379-1390.

[41] Thomas D Mueller and Joachim Nickel. "Promiscuity and specificity in BMP receptor activation”. In: FEBS letters 586.14 (2012), pp. 1846-1859.

[42] Joachim Nickel and Thomas D Mueller. "Specification of BMP signaling”. In: Cells 8.12 (2019), p. 1579.

[43] Oddrun Elise Olsen et al. "BMPR2 inhibits activin and BMP signaling via wild-type ALK2". en. In: J. Cell Sci. 131.11 (June 2018). ISSN: 0021-9533, 1477-9137. DOI: 10.1242/jcs. 213512 .

[44] Jeehae Park et al. "Dissecting the sharp response of a canonical developmental enhancer reveals multiple sources of cooperativity”. In: Elife 8 (2019), e41266. 
[45] D Russ and R Kishony. "Additivity of inhibitory effects in multidrug combinations". en. In: Nat Microbiol 3.12 (Dec. 2018), pp. 1339-1345. ISSN: 2058-5276. DOI: 10.1038/s41564018-0252-1.

[46] Valerie S Salazar, Laura W Gamer, and Vicki Rosen. "BMP signalling in skeletal development, disease and repair”. en. In: Nat. Rev. Endocrinol. 12.4 (Apr. 2016), pp. 203-221. ISSN: 1759-5029, 1759-5037. Dor: $10.1038 /$ nrendo.2016.12.

[47] Stefan Saremba et al. "Type I receptor binding of bone morphogenetic protein 6 is dependent on N-glycosylation of the ligand: Type I receptor specificity of BMP-6". en. In: FEBS J. 275.1 (Jan. 2008), pp. 172-183. ISSN: 1742-464X, 1742-4658. DOI: $10.1111 / \mathrm{j} .1742-$ $4658.2007 .06187 . \mathrm{x}$

[48] Monika Saxena, Nitin Agnihotri, and Jonaki Sen. "Perturbation of canonical and noncanonical BMP signaling affects migration, polarity and dendritogenesis of mouse cortical neurons". en. In: Development 145.1 (Jan. 2018). ISSN: 0950-1991, 1477-9129. DOI: 10. 1242 /dev. 147157.

[49] Bernhard Schmierer and Caroline S Hill. "TGF $\beta$-SMAD signal transduction: molecular specificity and functional flexibility". In: Nature reviews Molecular cell biology 8.12 (2007), pp. 970-982.

[50] Elad Schneidman et al. "Weak pairwise correlations imply strongly correlated network states in a neural population”. In: Nature 440.7087 (2006), pp. 1007-1012.

[51] Howard J Seeherman et al. "A BMP/activin A chimera is superior to native BMPs and induces bone repair in nonhuman primates when delivered in a composite matrix". en. In: Sci. Transl. Med. 11.489 (Apr. 2019). ISSN: 1946-6234, 1946-6242. DOI: $10.1126 /$ scitranslmed.aar4953.

[52] Petra Simic and Slobodan Vukicevic. "Bone morphogenetic proteins in development and homeostasis of kidney". In: Cytokine \& Growth Factor Reviews 16.3 (2005), pp. 299-308. ISSN: 1359-6101. DOI: $10.1016 / \mathrm{j}$. cytogfr.2005.02.010.

[53] David Smil et al. "Leveraging an Open Science Drug Discovery Model to Develop CNSPenetrant ALK2 Inhibitors for the Treatment of Diffuse Intrinsic Pontine Glioma". In: Journal of Medicinal Chemistry 63.17 (2020), pp. 10061-10085.

[54] Ping Sun et al. "BMP2/7 heterodimer is a stronger inducer of bone regeneration in periimplant bone defects model than BMP2 or BMP7 homodimer". In: Dental materials journal 31.2 (2012), pp. 239-248.

[55] Atsushi Suzuki et al. "Mesoderm induction by BMP-4 and-7 heterodimers". In: Biochemical and biophysical research communications 232.1 (1997), pp. 153-156.

[56] Benjamin Tajer et al. "BMP heterodimers signal via distinct type I receptor class functions". In: Proceedings of the National Academy of Sciences 118.15 (2021).

[57] Lei Tang. "Microbial interactions". In: Nature methods 16.1 (2019), pp. 19-19.

[58] Elif Tekin, Van M Savage, and Pamela J Yeh. "Measuring higher-order drug interactions: A review of recent approaches". In: Current Opinion in Systems Biology 4 (2017), pp. 16-23. 
[59] Yuji Teramura et al. "Single-molecule analysis of epidermal growth factor binding on the surface of living cells". In: The EMBO journal 25.18 (2006), pp. 4215-4222.

[60] Emmanuelle Tillet et al. "A heterodimer formed by bone morphogenetic protein 9 (BMP9) and BMP10 provides most BMP biological activity in plasma". In: Journal of Biological Chemistry 293.28 (2018), pp. 10963-10974.

[61] M R Urist. "Bone: formation by autoinduction". en. In: Science 150.3698 (Nov. 1965), pp. 893-899. ISSN: 0036-8075. DOI: 10.1126/science.150.3698.893.

[62] Elvira Valera et al. "BMP-2/6 heterodimer is more effective than BMP-2 or BMP-6 homodimers as inductor of differentiation of human embryonic stem cells". en. In: PLoS One 5.6 (June 2010), e11167. ISSN: 1932-6203. DOI: 10.1371/journal.pone.0011167.

[63] Ophelia S Venturelli et al. "Deciphering microbial interactions in synthetic human gut microbiome communities”. In: Molecular systems biology 14.6 (2018), e8157.

[64] Daniel M Weinreich et al. "The influence of higher-order epistasis on biological fitness landscape topography”. In: Journal of statistical physics 172.1 (2018), pp. 208-225.

[65] Eleanor Williams et al. "Saracatinib is an efficacious clinical candidate for fibrodysplasia ossificans progressiva". In: bioRxiv (2020).

[66] Pamela Yeh, Ariane I Tschumi, and Roy Kishony. "Functional classification of drugs by properties of their pairwise interactions". en. In: Nat. Genet. 38.4 (Apr. 2006), pp. 489-494. ISSN: 1061-4036. DOI: $10.1038 / \mathrm{ng} 1755$.

[67] Atilgan Yilmaz et al. "MuSK is a BMP co-receptor that shapes BMP responses and calcium signaling in muscle cells". en. In: Sci. Signal. 9.444 (Sept. 2016), ra87. ISSN: 1937-9145, 1945-0877. DoI: 10.1126/scisignal . aaf0890.

[68] Anat Zimmer et al. "Prediction of multidimensional drug dose responses based on measurements of drug pairs”. en. In: Proc. Natl. Acad. Sci. U. S. A. 113.37 (Sept. 2016), pp. 1044210447. ISSN: 0027-8424, 1091-6490. DOI: 10.1073/pnas. 1606301113. 